\title{
CsnA Dependent Development and Regulation of Amino Acid Biosynthesis of the Filamentous Ascomycete Aspergillus nidulans
}

\author{
Dissertation \\ zur Erlangung des Doktorgrades \\ der Mathematisch-Naturwissenschaftlichen Fakultäten \\ der Georg-August-Universität zu Göttingen
}

\author{
vorgelegt von \\ Oliver Draht \\ aus Helmstedt
}

Göttingen 2005 
Die vorliegende Arbeit wurde in der Arbeitsgruppe von Prof. Dr. Gerhard H. Braus in der Abteilung Molekulare Mikrobiologie und Genetik des Instituts für Mikrobiologie und Genetik der Georg-August-Universität Göttingen angefertigt.

Teile dieser Arbeit wurden veröffentlicht in:

Galagan, J.E., Calvo, S.E., Cuomo, C., Ma, L., Wortman, J., Batzoglou, S., Lee, S., Brudno, M., Bastürkmen, M., Spevak, C.C., Clutterbuck, J., Kapitonov, V.J., Scazzocchio, C., Farman, M., Butler, J., Purcell, S., Harris, S., Braus, G.H., Draht, O., Busch, S., D’Enfert, C., Bouchier, C., Goldman, G.H., Griffiths-Jones, S., Vienken, K., Pain, A., Selker, E.U., Archer, D., Penalva, M.Á., Oakley, B.R., Momany, M., Sano, M., Tanaka, T., Kumagai, T., Machida, M., Nierman, W.C., Denning, D.W., Caddick, M., Hynes, M., Paoletti, M., Fischer, E.R., Miller, J.B., Dyer, P., Sachs, M.S., Osmani, S.A., and Birren, B. (2005) Sequencing of Aspergillus nidulans and comparative analysis with A. fumigatus and A. oryzae. Manuscript submitted.

Pries, R., Bömeke, K., Draht, O., Künzler, M., and Braus, G.H. (2004) Nuclear import of yeast Gcn4p requires karyopherins Srp1p and Kap95p. Mol Genet Genomics 271: 257266.

D7

Referent: $\quad$ Prof. Dr. G.H. Braus

Korreferent: PD. Dr. S. Irniger

Tag der mündlichen Prüfung: 02.11 .2005 
Für meine Eltern und meine kleine Rose 



\section{Abbreviations}

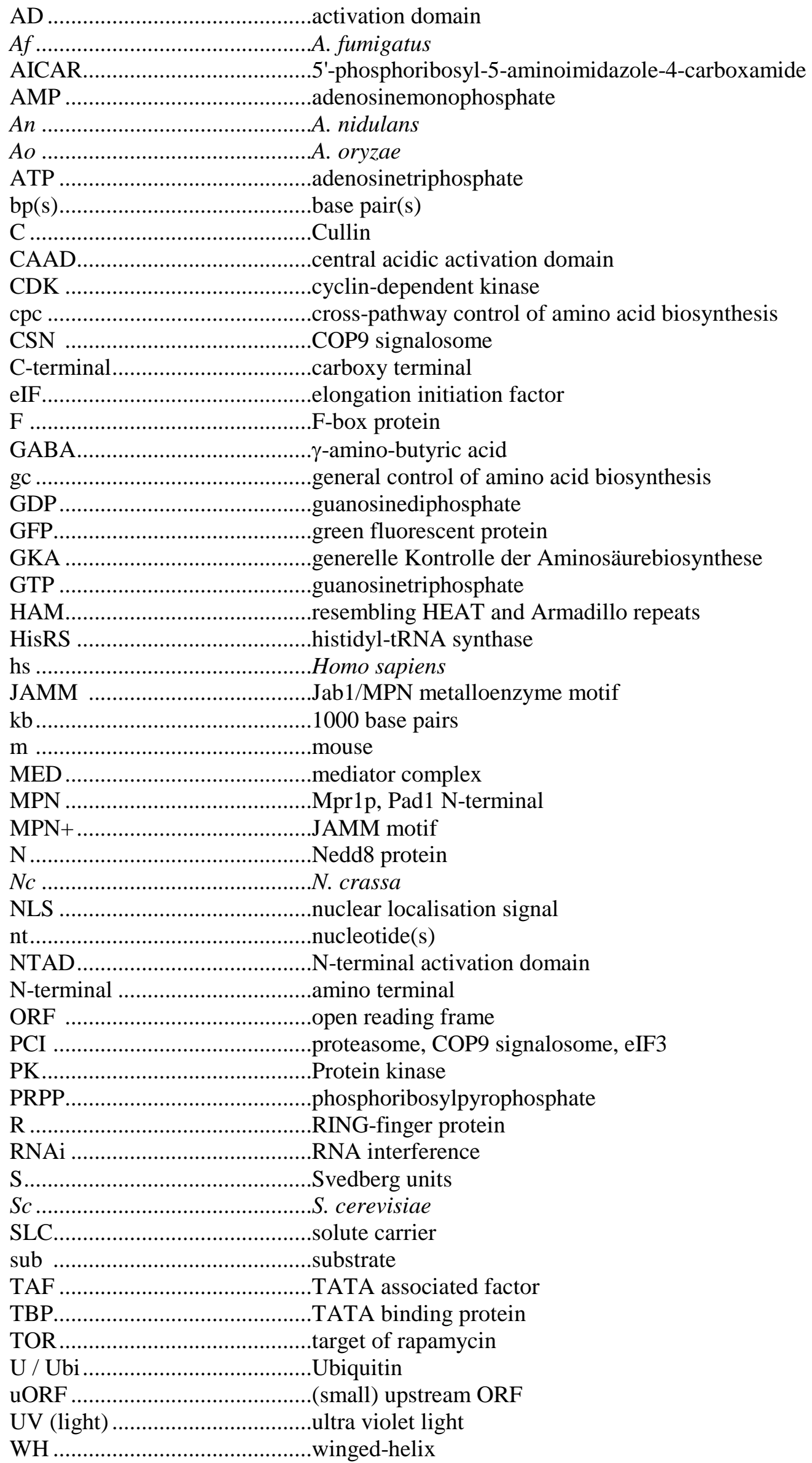



Summary 1

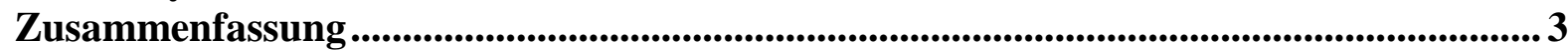

\section{Chapter 1}

Introduction

1.1 Genetic control of COP9 dependent development and cross-pathway control..................5

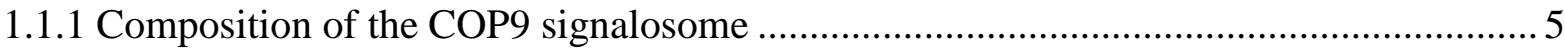

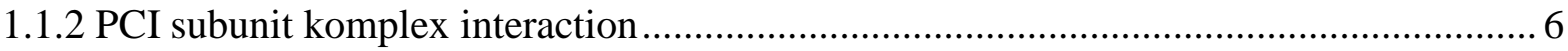

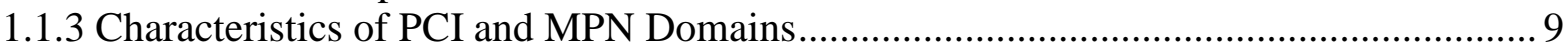

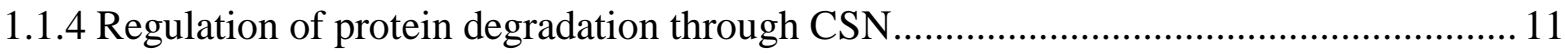

1.1.5 The role of CSN in control of cellular functions................................................... 15

1.2 Control of amino acid biosynthesis in fungi .......................................................... 17

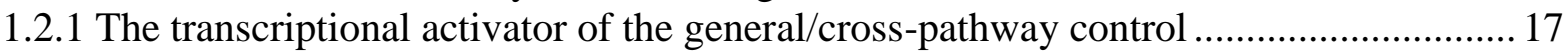

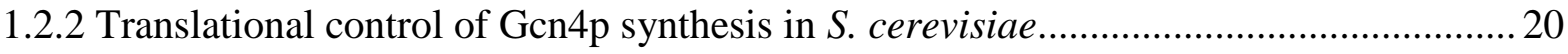

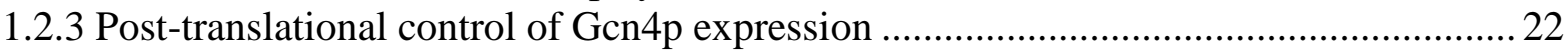

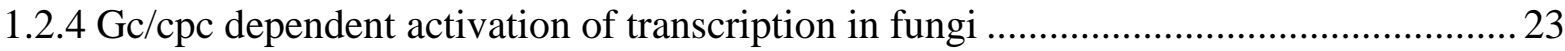

1.2.5 Impact of the cross-pathway control on A. nidulans development .............................. 25

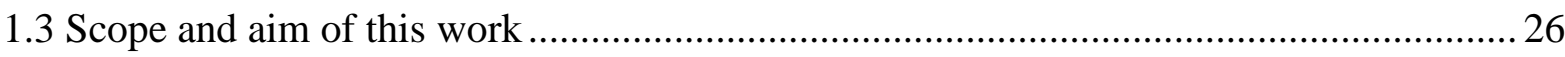

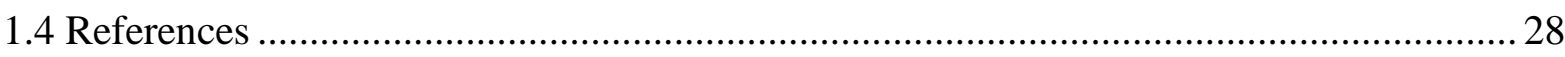

\section{Chapter 2}

Aspergillus nidulans sexual development is dependent on

COP9 deneddylation activity ...................................................................................................41

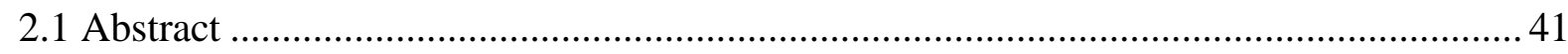

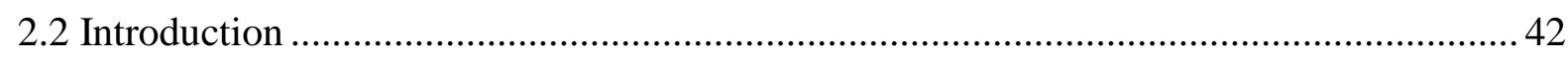

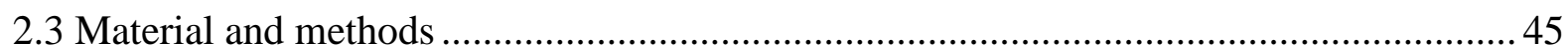

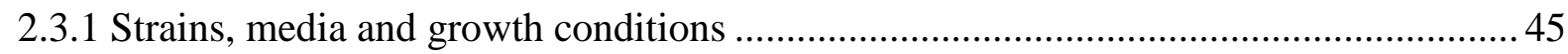

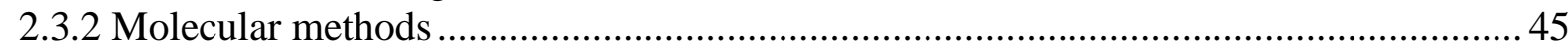

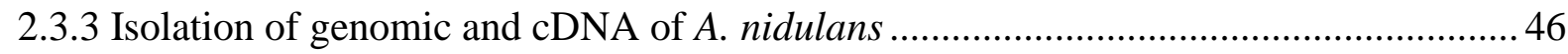

2.3.4 Construction of plasmids for A. nidulans manipulation............................................. 46

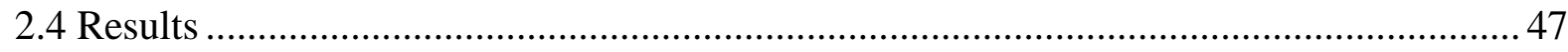

2.4.1 The A. nidulans $\operatorname{csnA}$ gene encodes a PCI domain protein similar to subunit I of the COP9 signalosome.

2.4.2 Aspergillus nidulans strains deleted for $\operatorname{csn} A$ are blocked in sexual development and produce altered secondary metabolites ........................................................... 50

2.4.3 Expression of truncated CsnA peptides can restore wild type secondary metabolism and sexual development in A. nidulans csnA deletion strains 
2.4.4 COP9 mutants display a growth defect in vegetative mycelium on solid medium at $37^{\circ} \mathrm{C}$

2.4.5 Structural in silico analysis of the investigated truncated CsnA proteins of $A$. nidulans

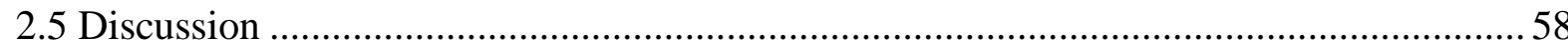

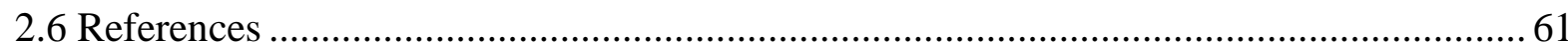

\section{Chapter 3}

Annotation of cross-pathway control related genes in the genome of Aspergillus nidulans, Aspergillus fumigatus and Aspergillus oryzae ................................ 64

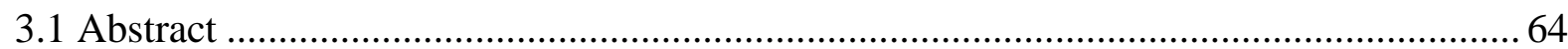

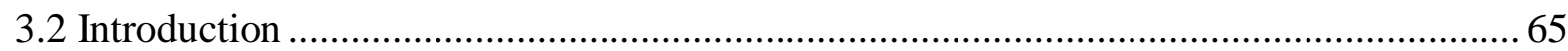

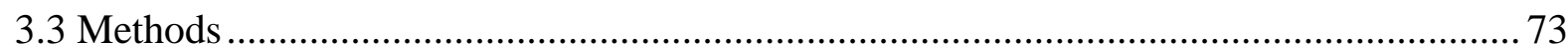

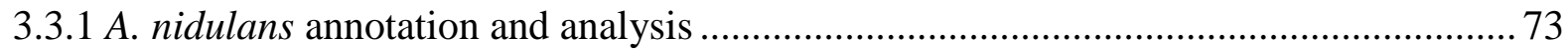

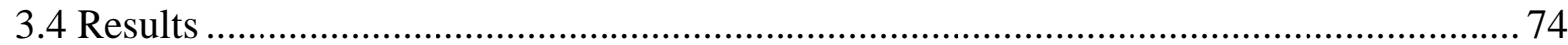

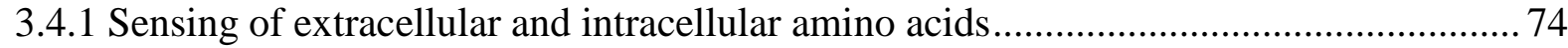

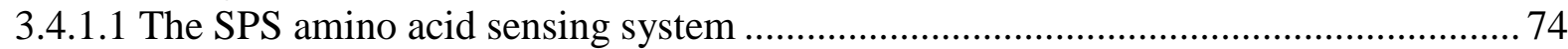

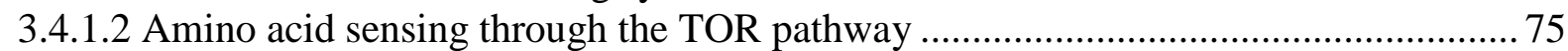

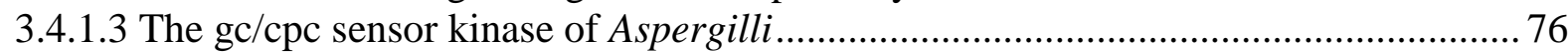

3.4.2 Uptake of amino acids in the filamentous fungus $A$. nidulans ................................... 76

3.4.2.1 Amino acid uptake systems of Aspergillus species............................................... 76

3.4.2.2 Aspergillus amino acid uptake systems in comparison to mammalian counterparts .. 78

3.4.3 Regulation of fungal protein biosynthesis in response to intracellular

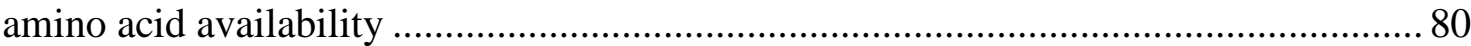

3.4.3.1 Regulation of expression of the central transcription factor of $\mathrm{gc} / \mathrm{cpc}$

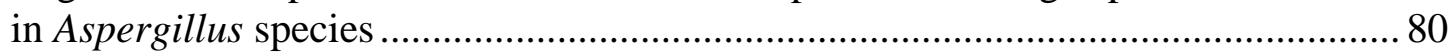

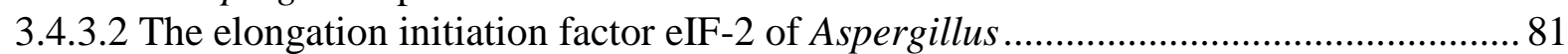

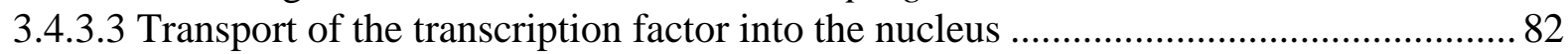

3.4.3.4 Regulation of the protein level of the central transcription factor of the $\mathrm{gc} / \mathrm{cpc} . . . . . . .82$

3.4.4 Fungal amino acid biosynthesis investigated on selected examples .......................... 85

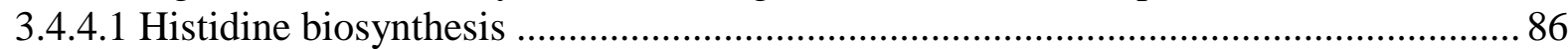

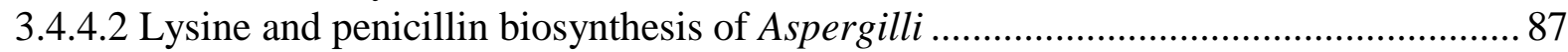

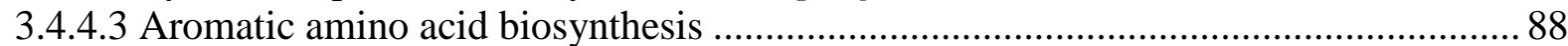

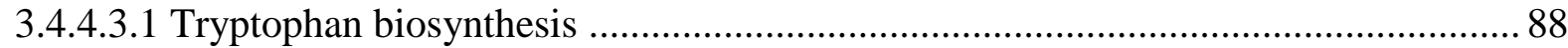

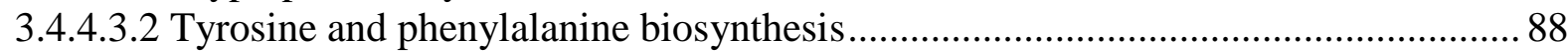

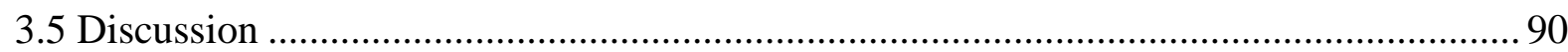

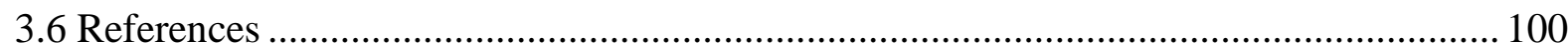




\section{Chapter 4}

A. nidulans CpcA localisation and nuclear localisation signal (NLS)............................... 109

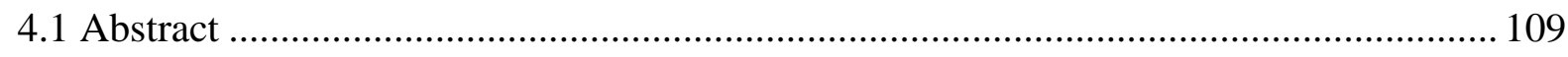

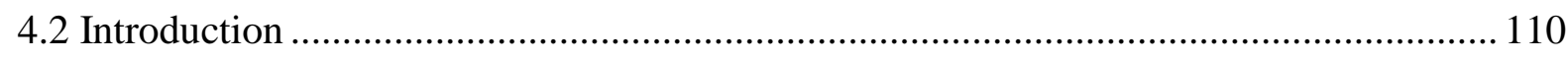

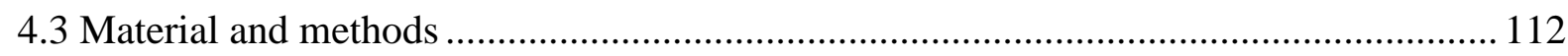

4.3.1 Transformation and cultivation of Aspergillus nidulans ........................................... 112

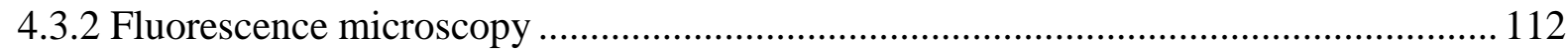

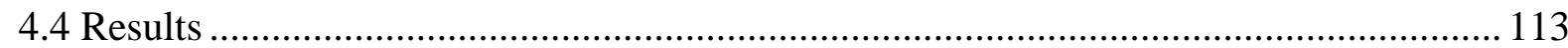

4.4.1 Comparison of Gen $4 p$ and CpcA reveals that only NLS2 is conserved between Saccharomyces cerevisiae and Aspergillus species ...................... 113

4.4.2 Deletion of the conserved nuclear localisation sequence in $\mathrm{CpcA}$ of Aspergillus nidulans impairs its nuclear transport .................................................... 113

4.4.3 In silico investigation on putative importins of Aspergillus nidulans......................... 116

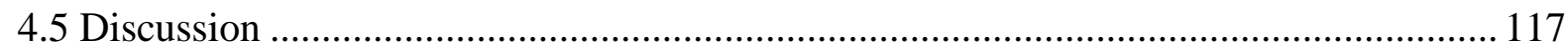

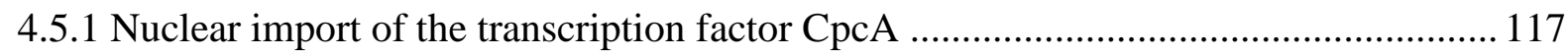

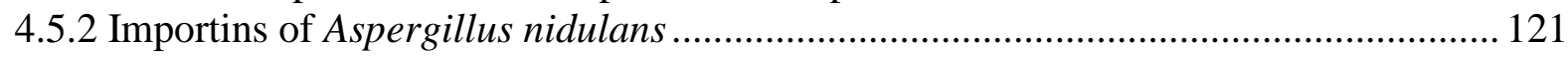

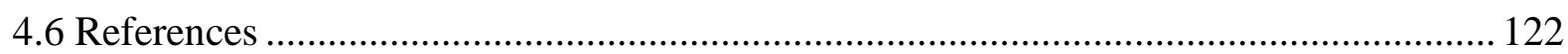





\section{Summary}

Aspergilli comprise a heterogenous group of filamentous fungi. The ascomycete Aspergillus nidulans represents a well studied eukaryotic model system for metabolism and development. Aspergillus oryzae and the opportunistic human pathogen A. fumigatus are deuteromycetes with significant impact on asian food industry and medical research respectively. This work focusses on the COP9 signalosome as an essential regulator of development in higher eukaryotes and the cross-pathway control of amino acid biosynthesis which regulates the cellular response to amino acid starvation conditions. Both networks are well conserved from yeast to human.

The COP9 signalosome is a multiprotein complex with two major associated enzyme activities - associated kinase and de-ubiquitination activities and an additional intrinsic deneddylation activity. In this thesis, the relevance of the first subunit CsnA of the COP9 signalosome is investigated by expression of truncated CsnA peptides in $\operatorname{csn} A$ deletion strains. A deletion in $\operatorname{csn} A$ results in multiple pleiotrophic phenotypes. Expression of the truncated CsnA proteins made it possible to separate functions of the COP9 holoenzyme from CsnA functions. The C-terminal part of CsnA seems to be essential for integration into the COP9 signalosome and maintaining the structural integrity and activities of the complex. The $\mathrm{N}$-terminal of CsnA seems to play a role in regulation of formation of aerial hyphae.

During the course of the manual annotation of the genomes of the three fungi A. nidulans, A. fumigatus and A. oryzae the constituent genes of the cross-pathway control of amino acid biosynthesis $(c p c)$ in comparison to higher and lower eukaryotes were investigated. The results show that basic mechanisms of the $c p c$ of these filamentous fungi resemble those of yeast and are less complex than in higher eukaryotes. The external and internal amino acid sensing and uptake system of amino acids rather resembles that of mammals than of unicellular yeasts. This indicates a possible role of amino acid uptake systems in regulation of cellular growth and development similar to that of mammalian cells.

The transport of the central transcription factor $\mathrm{CpcA}$ into the nucleus was investigated in S. cerevisiae and in A. nidulans. Efficient transport to the nucleus requires the nuclear localisation signal. In S. cerevisiae two importins Srp1p and Kap95p were identified to be essential for transport of $\mathrm{CpcA}$ into the yeast nucleus. In silico investigations in the Aspergillus genomes revealed the highly similar proteins SrpA and KapA that might be involved in nuclear transport of CpcA in A. nidulans. 



\section{Zusammenfassung}

Die Familie der Aspergillen ist eine sehr heterogene Gruppe filamentöser Pilze. Aspergillus nidulans ist ein wichtiger eukaryotischer Modellorganismus für Metabolismus- und Entwicklungsstudien. Aspergillus oryzae ist von großer biotechnologischer Bedeutung in der asiatischen Lebensmittelindustrie, wohingegen der opportunistische humanpathogene Pilz A. fumigatus von wachsender medizinischer Bedeutung ist. Diese Arbeit konzentriert sich auf das COP9 Signalosom, einen essentiellen, eukaryontischen Entwicklungsregulator und die generelle Kontrolle der Aminosäurebiosynthese (gc). Die generelle Kontrolle regelt die zelluläre Antwort auf Aminosäuremangel. Beide Netzwerke sind von der Bäckerhefe bis zum Menschen hoch konserviert.

Das COP9 Signalosom ist ein Multiproteinkomplex, dessen wichtigste zugehörige enzymatische Aktivitäten aus Kinase- und De-ubiquitinierungsaktivitäten und einer intrinsischen Deneddylaseaktivität bestehen. Diese Arbeit untersucht die Relevanz der ersten Untereinheit des COP9 Signalosoms CsnA anhand der Expression von verkürzten Proteinvarianten. csnA Deletionsmutanten weisen diverse pleiotrophe Phänotypen auf. Die Expression von verkürzten CsnA Peptiden ermöglichte es, die Funktionen des COP9 Signalosoms von Aktivitäten des CsnA Proteins zu trennen. Der CsnA C-Terminus ist wichtig für die Aufrechterhaltung des Komplexzusammenhalts und der COP9 Aktivitäten. Der CsnA N-Terminus hingegen scheint eine wichtige Rolle in der Regulation der Ausbildung von Lufthyphen zu spielen.

Im Zuge der manuellen Annotation der Genome der Pilze A. nidulans, A. fumigatus und A. oryzae wurden grundlegende Gene der generellen Kontrolle der Aminosäurebiosynthese (cpc) im Vergleich zu niederen und höheren Eukaryonten untersucht. Die Resultate der Untersuchung zeigen, daß die grundlegenden Mechanismen der cpc der filamentösen Pilze eher denen der Hefen ähneln und etwas weniger komplex als bei höheren Organismen sind. Die externen und internen Sensoren und die Aufnahmesysteme für Aminosäuren hingegen scheinen eher denen höherer Eukaryonten zu ähneln als denen einzelliger Hefen. Die Ergebnisse weisen darauf hin, daß die Aufnahme- und Sensorsysteme für Aminosäuren einen Einfluß auf das Wachstum und die Entwicklung der Pilze haben könnten, wie man sie aus Säugersystemen kennt.

Die Untersuchung des Transports des zentralen Transkriptionsfaktors der cpc CpcA in den Zellkern wurde in S. cerevisiae und A. nidulans untersucht. Für einen effizienten Transport in den Nukleus ist das Kernlokalisierungssignal von $\mathrm{CpcA}$ notwendig. In der Bäckerhefe wurden zwei Importine Srp1p und Kap95p identifiziert, die essentiell für den Kerntransport von CpcA sind. Diese wurden auch hoch konserviert in in silico Untersuchungen in den Aspergillengenomen wiedergefunden. Die hypothetischen Aspergillenproteine SrpA und KapA könnten auch in Aspergillus notwendig für den Kerntransport von CpcA sein. 



\section{Chapter 1}

\section{Introduction}

\subsection{Genetic control of COP9 dependent development and cross-pathway control}

In living organisms cellular actions need to be closely regulated. This chapter will focus on two aspects of genetic regulation in cells. Each cell needs amino acids as essential building blocks for protein synthesis. Amino acids can either be taken up from the diet or culture medium or synthesized de novo by the cells. The de novo synthesis of amino acids in fungi is tightly controlled by the general or cross-pathway control of amino acid biosynthesis. Another way of obtaining amino acids is the degradation of proteins in the cell. The degradation needs to be highly controlled as well to degrade only proteins that are not longer needed. One of the major regulators of the protein degradation machinery is the COP9 signalosome, a multi-subunit complex that controls the activity of the SCF and thus the targeting of proteins for degradation.

\subsubsection{Composition of the COP9 signalosome}

The COP9 signalosome, or CSN, was originally identified in a screen for altered light response loss-of-function mutants of Arabidopsis thaliana and later in other higher and lower eukaryotes (Bech-Otschir et al., 2002; Busch et al., 2003; Chamovitz and Segal, 2001; HarariSteinberg and Chamovitz, 2004; Irniger and Braus, 2003; Kim et al., 2001; Schwechheimer and Deng, 2001; Seeger et al., 2001; Wei et al., 1994). In the screen two groups of mutants were found: the ones that exhibit light-grown seedling characteristics in the absence of light, a constitutive photomorphogenesis (cop) and the other group of mutants that showed deethiolation (det) (Wei and Deng, 1992). Lethal mutants in this class are allelic to fusca mutants which accumulate anthocyanin, a purple pigment, in the mature seed coat and the embryonic leaves (Gusmaroli et al., 2004; Misera et al., 1994). These mutants were classed as cop/detlfus mutants in A. thaliana and later on it could be shown that their respective gene loci coded for six of the eight subunits of the COP9 signalosome (Schwechheimer and Deng, 2001; Wei et al., 1998). The mammalian CSN complex is also known as the JAB containing signalosome according to the fifth subunit JAB1 (Carrabino et al., 2004). It was originally 
identified as a copurifying byproduct of the 26S proteasome (Scheel and Hofmann, 2005; Seeger et al., 1998).

The overall identification of multi-species CSN complexes from yeast to mammals revealed evolutionary evidence of the conservation of this complex and other PCI complexes. The CSN shares high homology to the catalytic 19S subunit proteins of the proteasome lid and to the eukaryotic translation initiation factor eIF3 subunits. The 26S proteasome, which is required for the degradation of ubiquitinated proteins, consists of the $20 \mathrm{~S}$ core particle and two 19S lid components located at the exterior ends of the core particle. The eIF3 function is to prevent premature association of the $40 \mathrm{~S}$ and $60 \mathrm{~S}$ ribosomal subunits. It facilitates the loading of the 40S subunit onto the ternary eIF2-tRNA-Met-GTP complex and interacts with other translation factors. These three complexes contain proteins with a similar interaction

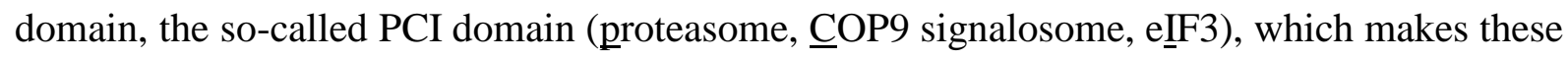
three complexes to PCI protein complexes. A unified nomenclature of the CSN subunit orders them by decreasing size from CSN1 to CSN8 (Deng et al., 2000) (Table 1). In CSN six of the eight subunits harbor PCI domains, whereas two of the subunits harbor MPN domains (Mpr1p, Pad1 N-terminal). The average size of PCI domains is about 140 amino acids and 200 amino acids for MPN domains. Each of the COP9 signalosome subunits share pair-wise similarities to the subunits of the $19 \mathrm{~S}$ proteasome lid, indicating that they are homologous with a common evolutionary ancestor. The eIF3 complex, on the other hand, contains three PCI and two MPN proteins among its 11 components (Kim et al., 2001).

\subsubsection{PCI complex subunit interaction}

Subunit interactions of the CSN are largely conserved. Investigations on subunit interactions in several species made it possible to map the interactions of the subunits, summarized in Figure 1 (Fu et al., 2001; Kapelari et al., 2000; Serino et al., 2003; Tsuge et al., 2001). Electron microscopy studies on the hsCSN and lid complex revealed a similar shape, that lacks symmetry and has a central groove structure (Kapelari et al., 2000). Apart from the eight subunit CSN complex smaller partial complexes, whose relevance is so far unknown, have been identified. Small portions of CSN4 and CSN7 were found independently of CSN1 in Arabidopsis (Karniol et al., 1999; Serino et al., 1999; Wang et al., 2002) and Drosophila (Oron et al., 2002). In S. pombe complexes containing CSN4 and CSN5 were found (Mundt 
et al., 2002). Additionally, a cytoplasmic-localized subcomplex consisting of CSN4 to CSN8 was found in mammals (Tomoda et al., 2002).

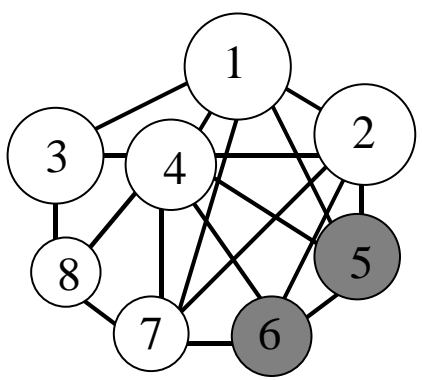

Fig. 1: Subunit interactions in the CSN complex

The figure shows a summary of reported interactions of the CSN subunits. The PCI domain containing subunits are displayed in white, MPN domain containing subunits are displayed in grey (Wei and Deng, 2003).

The interaction between CSN subunits was shown to require PCI domains, which assumably play a scaffolding role in the assembly of the multiprotein complex. So far PCI domain proteins have only been reported in eukaryotic organisms not in prokaryotes, whereas MPN domain proteins were also found in prokaryotes. The MPN domains are also known as JAMM (Jab1/MPN domain metalloenzyme) or MPN+ motif. The JAMM motif is responsible for the catalytic cleavage of the Nedd8-cullin conjugate by the CSN (Ambroggio et al., 2004). The dynamic and cleavage of Nedd8 is responsible for activity regulation of the SCF complex (Pan et al., 2004). The JAMM domain itself is embedded in the large MPN domain. These two types of domains are found predominantly among components of the three large protein complexes, the CSN, the proteasome lid and the eukaryotic translation initiation factor eIF3 (Ambroggio et al., 2004; Glickman et al., 1998; Hofmann and Bucher, 1998; Wei et al., 1998). The subunits of the three complexes not only show similarities, but it was found that the CSN subunits even interact physically with the other PCI complexes, which was shown in copurifications and two-hybrid assays (Karniol et al., 1998; Seeger et al., 1998; Wei and Deng, 2003). Subunit CSN1 of A. thaliana was found to bind to RPN6 and eIF3c in yeast two-hybrid assays (Karniol et al., 1998; Kwok et al., 1999). eIF3i was shown to interact strongly with CSN3, CSN6 and CSN7, but only weakly with CSN1 and CSN8 (Hoareau Alves et al., 2002; Yahalom et al., 2001). In S. cerevisiae the lid subunit Rpn5p was found to bind stably to Csn5p, additionally Pci8p (CSN11) interacts with eIF3 subunits (Peng et al., 2001c; Shalev et al., 2001). Due to the strong similarities between the single subunits and their interactions it has been proposed that the CSN might constitute an alternative lid for the proteasome (Schwechheimer and Deng, 2001). 


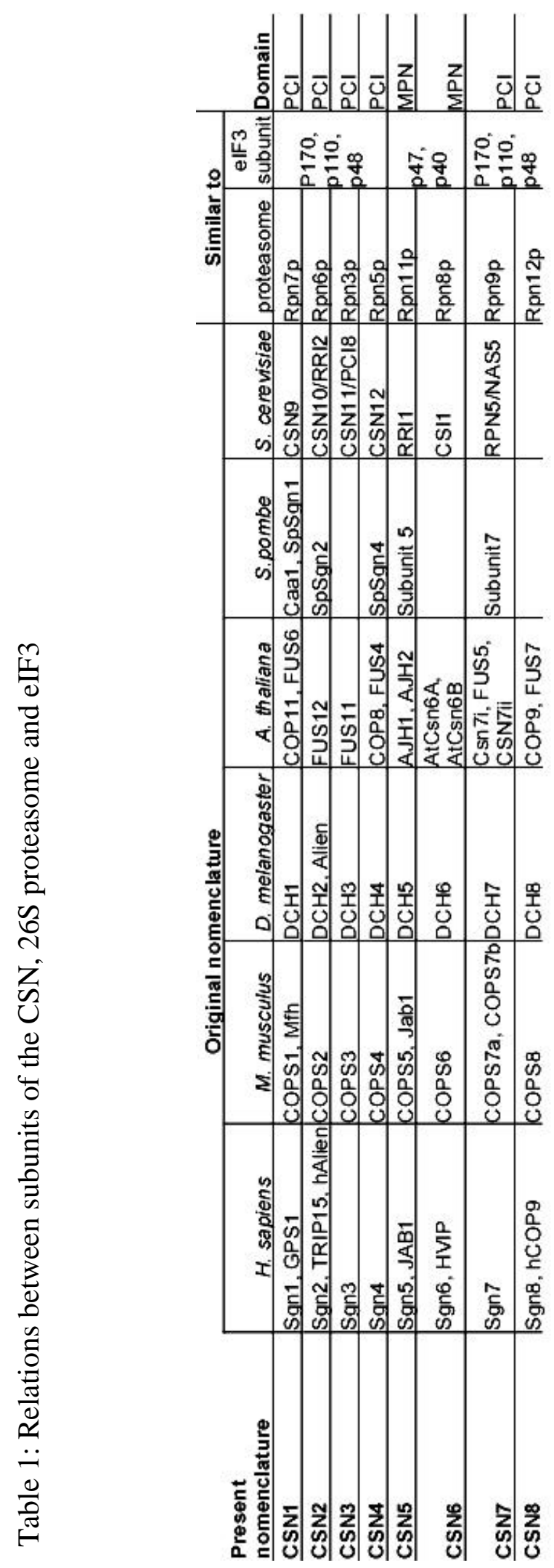




\subsubsection{Characteristics of PCI and MPN domains}

PCI or PINT domains are found in three multiprotein complexes, the proteasome lid, the COP9 signalosome and the eukaryotic translation initiation factor eIF3. There are several hints, that suggest that the PCI subunits are crucial for complex formation, so far no catalytic activity was described for them (Freilich et al., 1999; Lier and Paululat, 2002; Tsuge et al., 2001; Valasek et al., 2001). The MPN subunits are well conserved and the bioinformatic detection of the domains and their boundaries is relatively simple. In contrast the detection of PCI domains is troublesome due to their high degree of divergence.

The sequence similarities which suggest homology between single subunits of CSN and proteasome lid are easy to spot, whereas the detection of similarities between other paralogous PCI subunits requires advanced approaches, like the generalized profile method (Bucher et al., 1996; Hofmann and Bucher, 1998). Due to these difficulties it is to be expected that a portion of PCI domain proteins remains hidden in the depth of eukaryotic genomes up to now.

A second difficulty arises when it comes to assigning boundaries to the PCI domains. In general one regards a homology domain as a structural unit. This can be regarded true for the C-terminal part of PCI domains, but the amino acid sequence forming the N-terminal part of the PCI domain rather gradually fades instead of providing a sharp drop to provide a sharp boundary. Therefore various domain databases define the N-terminal boundary for PCI domains differently. Recent bioinformatic studies revise the point that a PCI domain should be regarded as an homology domain, but rather consists of two structural distinct domains (Scheel and Hofmann, 2005). The boundaries of the C-terminal part of the PCI domain are well defined by a notable loss of sequence similarities, whereas the boundaries of the N-terminal portion of the PCI domain are difficult to define, because the sequence conservation of this region is low and different families of PCI domain proteins lose their similarity at different positions (Scheel and Hofmann, 2005).

The actual PCI domain can be separated into two domains, the C-terminal WH-domain and the N-terminal HAM domain. The WH-domain is a globular $\alpha / \beta$ structure with an “ $\alpha \beta \alpha \alpha \beta \beta$ "-arrangement, which can be classified as a winged-helix (WH) motif. The HAM domain is entirely helical with a core of six regularly-spaced helices that form three antiparallel helical hairpins. It resembles structurally mainly HEAT and Armadillo-repeats, creating the name HAM-domain. Scheel and Hofmann found that TPR-like (tetratrico-peptide repeats) repeats precede many PCI domains, which consist of short bi-helical segments. The 
data provided by Scheel and Hofmann and Tsuge et al. indicate that different parts of the PCI domain are responsible for binding of different binding partners: a truncated Csn1 protein of A. thaliana containing the full PCI domain was able to bind to Csn2, Csn3 and Csn4, whereas a truncated Csn 1 protein lacking the WH-region was only able to bind to Csn4 and a truncated protein lacking the helical-repeat region was only able to bind to Csn3. Similar results have been found for proteasome subunits, where amino acid substitutions as a result of point mutations in the WH-portion or the helical-repeat portion of PCI domain proteins were found to abrogate binding to other subunits (Isono et al., 2004).

Whilst no catalytic activity was assigned to PCI domain proteins so far, these proteins must have at least three distinct functions: maintaining the structural integrity of the PCI complexes, attaching the MPN subunit to the complexes and binding to other complexes like eIF3 subunits or the proteasome lid (Karniol et al., 1998; Kwok et al., 1999; Peng et al., 2003; Shalev et al., 2001).

The MPN domain appears to be more ancient and diverse than the PCI domain and was found in non-complexed independent proteins and even in prokaryotes (Maytal-Kivity et al., 2002b). MPN domain proteins harbor the JAMM or MPN+ motif (JAB1/MPN/Mov34 metalloenzyme). The MPN+ motif contains five polar residues that resemble the active site residues of hydrolytic enzyme classes, particularly that of metalloproteases of the following composition $\mathrm{EX}_{\mathrm{n}} \mathrm{HS} / \mathrm{THX}_{7} \mathrm{SXXD}$, coordinating a zinc ion, whereas the glutamic acid residues forms hydrogen-bonds to a water ligand (Ambroggio et al., 2004). The MPN+ domain is embedded in the larger JAMM domain in some MPN proteins. The CSN harbors two MPN domain proteins, CSN5 and CSN6, the proteasome harbors Rpn11p and Rpn8p (MaytalKivity et al., 2002b). CSN5 and Rpn11p, but not CSN6 and Rpn8p contain a JAMM/MPN+ motif in their respective MPN domain (Maytal-Kivity et al., 2002b). The two MPN domain proteins of the eukaryotic translation initiation factor eIF3 do not have the JAMM/MPN+ motifs. The function of MPN proteins lacking the MPN+ motif so far remains unclear, but they are obviously necessary for interactions between subunits of these complexes (MaytalKivity et al., 2002b).

The MPN+ motif in CSN5 constitutes for the catalytic isopeptidase activity for the cleavage of Nedd8 protein from Cul1 by the CSN or CSN-like complexes (Cope et al., 2002), whereas Rpn11p, a subunit of the proteasome, constitutes the major de-ubiquitination activity of the 26S proteasome (Verma et al., 2002; Yao and Cohen, 2002). DNA point mutations leading to changes in conserved amino acid residues of either of the proteins MPN+ domains lead to a loss of function of the respective isopeptidase activity (Ambroggio et al., 2004; Maytal-Kivity 
et al., 2002b). Interestingly the two proteins exhibit their isopeptidase activity only if they are assembled to their respective complexes, allowing an efficient regulation of the catalytic activities of the proteins (Cope et al., 2002).

\subsubsection{Regulation of protein degradation through CSN}

Since its initial discovery as a regulator of light-morphogenesis in A. thaliana many studies in different eukaryotic species have shown the CSN to be a key regulator of cellular mechanisms and development. The CSN exhibits its regulation mainly through its CSN5 associated metalloprotease activity and signalosome associated kinase activities play a regulatory role in protein stabilisation and destabilisation through phosphorylation (Figure 2).

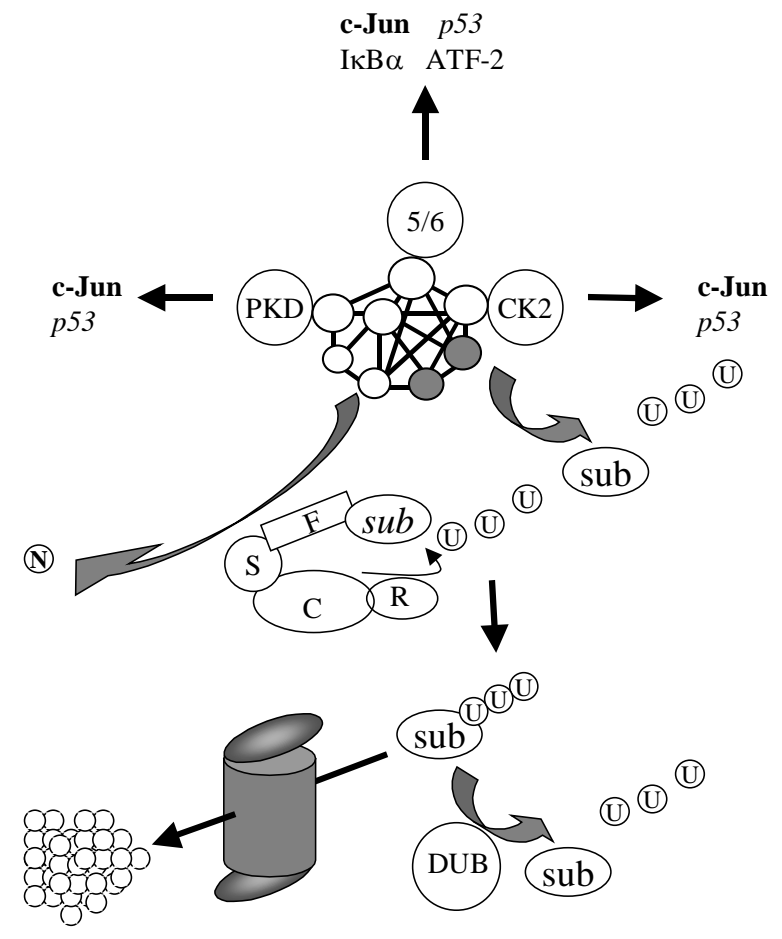

Fig. 2: The $\mathrm{CSN}$ is a central regulator of protein degradation

Associated CSN kinases phosphorylate protein targets like the transcription factors c-JUN and p53 and mark them either for degradation (italic) or stabilisation in the cell (bold) (Uhle et al., 2003; Zheng et al., 2002). Further on the CSN deneddylates and thus activates the SCF complex (Hoffmann et al., 1999; Hoffmann et al., 2000; Tomoda et al., 2004; Valerius et al., 2001). The SCF complex ubiquitinates bound substrates and marks them for degradation in the $26 \mathrm{~S}$ proteasome. Deubiquitylation enzymes (DUBs) and the CSN are able to rescue the substrate from destruction by removing the ubiquitin marking (Berndt et al., 2002; Grundmann et al., 2001; Zhou et al., 2003). A more detailed description is found in the text. 
The major target of the CSN is the cullin of the SCF ubiquitin ligase complexes. The SCF is an E3 enzyme that conjugates ubiquitin to its target proteins and thus targets them for degradation in the $26 \mathrm{~S}$ proteasome. A typical SCF complex consists of CUL1, a member of the cullin family, a small RING-finger protein Rbx1/Roc1/Hrt1, Skp1 and an F-box protein (Deshaies, 1999). So far three other cullin proteins next to the CUL1 homologue Cdc53p have been found in S. cerevisiae: Apc2p mediating the securin ubiquitination at the onset of sisterchromatid separation in mitosis (Tang et al., 2001), Cul8p which is needed for anaphase progression (Michel et al., 2003) and Cul3p with so far unknown function (Laplaza et al., 2004; Michel et al., 2003). F-box proteins specifically bind substrates following their phosphorylation in response to activation of various signaling pathways (Meimoun et al., 2000; Tyers and Jorgensen, 2000). In addition to the CUL1, five other cullins have been found in human, two of which can also be found in S. pombe, but do not have orthologs in S. cerevisiae where three cullins are found (Kominami et al., 1998). All human cullins interact with the HRT1/RBX1/ROC1 RING-finger proteins and have a ubiquitin ligase activity in vitro (Ohta et al., 1999; Ohta and Xiong, 2001).

The activity of the SCF complexes is regulated through covalent modification of the CUL1 subunit through attachment of the ubiquitin-like peptide Nedd8/Rub1. Covalent binding of Nedd8 or Rub1 to the cullin 1 subunit of the SCF, or neddylation, occurs through a pathway very similar to the ubiquitin ligating pathway: it is catalyzed by an enzymatic cascade involving Nedd8-activating enzymes APP-BP1 and Uba3 (E1) and the conjugating enzyme Ubc12 (E2) (Hershko and Ciechanover, 1998; Hochstrasser, 2000). The neddylation pathway is essential in yeast, worm and mouse and plays a significant role in auxin response in plant (del Pozo et al., 2002; Osaka et al., 2000; Tateishi et al., 2001).

Removal or deneddylation of the Nedd8 peptide from the cullin subunit is carried out by the metalloprotease activity of the CSN5 subunit of the COP9 signalosome, interestingly noncomplexed CSN5 subunits do not exhibit this catalytic activity (Cope et al., 2002). The CSN was found to bind to CUL1 and Rbx1 via CSN2, CSN6 and CSN1's N-terminal domain (Lyapina et al., 2001; Schwechheimer et al., 2001; Yang et al., 2002) and promotes the SCF function in vivo. Paradoxically deneddylation of the cullin 1 subunits inhibits SCF activity in vitro (Cope and Deshaies, 2003; Wei and Deng, 2003; Wolf et al., 2003). A possible solution for this paradoxon is that the SCF tends to auto-ubiquitination of the SCF components and thus leads to degradation of the SCF instead of the target proteins (Figure 3 ). In wildtype cells the SCF-bound substrate is poly-ubiquitinated and further degraded at the 
26S proteasome, during this time the cullin subunit remains neddylated (He et al., 2005; Wee et al., 2005).

A

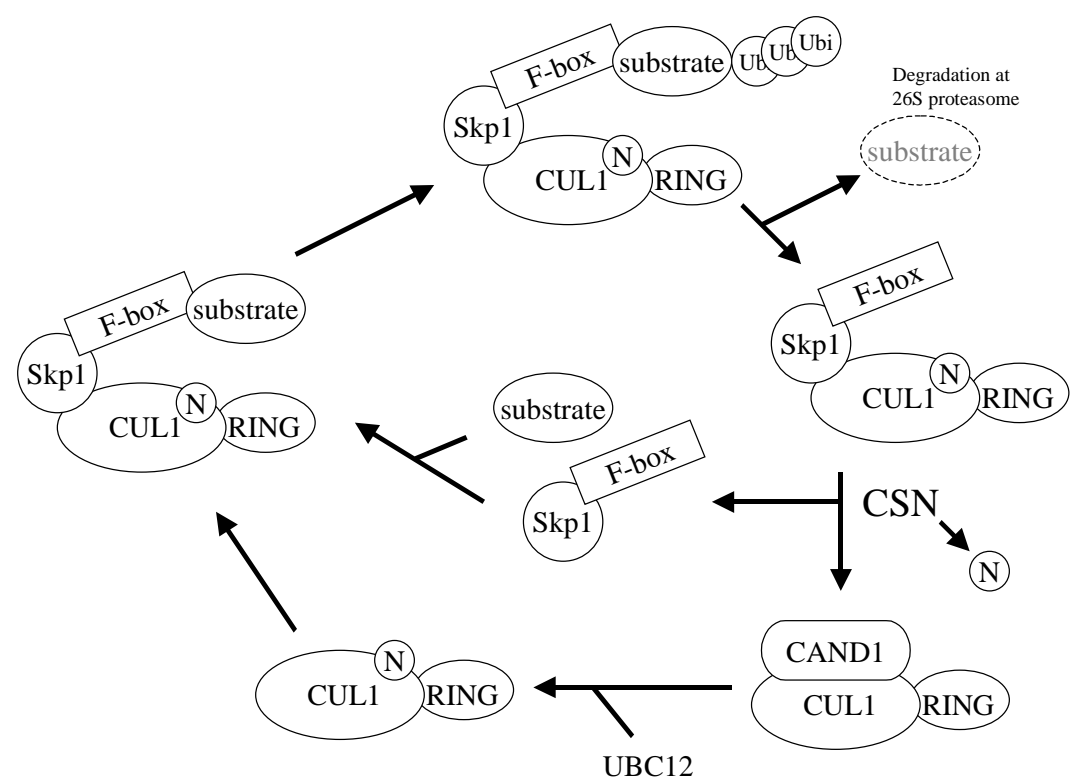

B

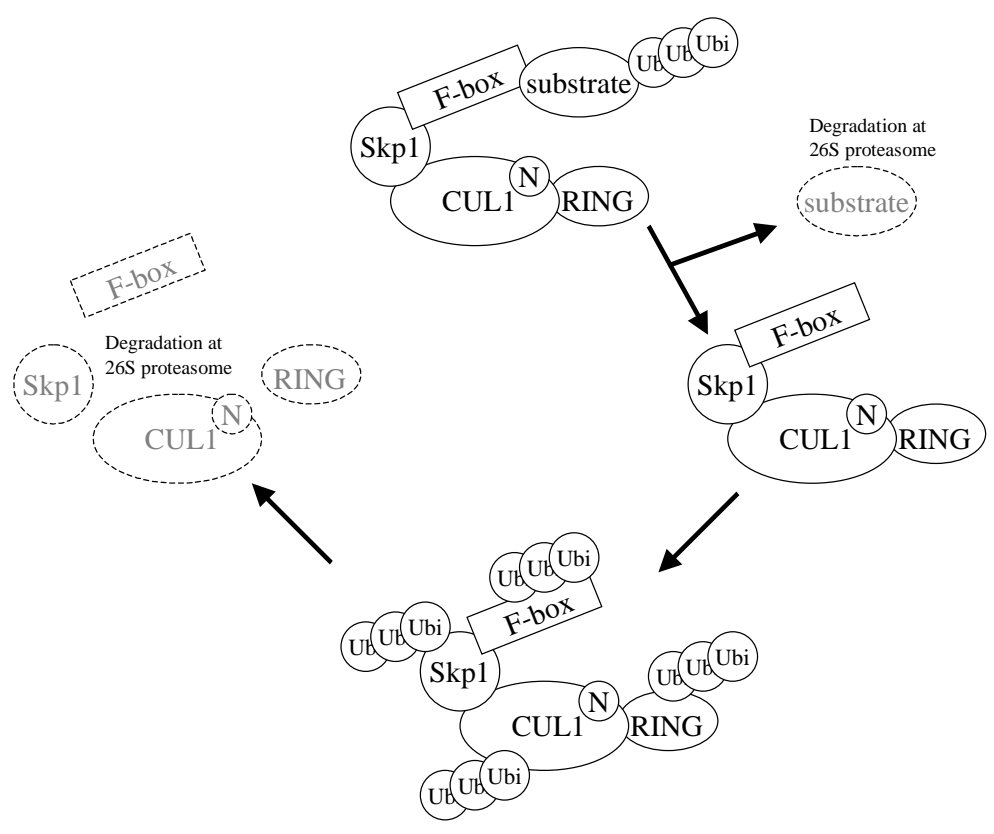

Fig. 3: Regulation of the SCF cycle

(A) In a wild type eukaryotic cell the assembled, substrate bound SCF ubiquitinates its substrate, which is then degraded in the $26 \mathrm{~S}$ proteasome. Subsequently the SCF is disassembled after deneddylation by the CSN and CAND1 binds to the cullin subunit rendering the ligase complex inactive. During re-neddylation of the cullin and dissociation of CAND1 from the cullin subunit the Skp1/F-box subunits are recharged with a new substrate and the SCF is reassembled. In $c s n$ mutants (B) the complex fails to disassemble after ubiquitination of the substrate and its subunits are subject to auto-ubiquitination and destruction in the $26 \mathrm{~S}$ proteasome (He et al., 2005). 
In HeLa cells it was found, that the deneddylated cullin and RING-finger part of the SCF are bound by CAND1 (cullin associated and Nedd8 dissociated). This leads to dissociation of the Skp1/F-box part from the SCF and inactivation of the ubiquitin ligase (Zheng et al., 2002). During the dissociated state of the Skp1/F-box proteins these are recharged with new substrates for ubiquitination. Ubc12 neddylates the cullin subunit, which in turn leads to dissociation of CAND1 and re-association of the substrate bound Skp1/F-box proteins. A defect in the deneddylation activity leads to degradation of the target protein, but leaves the neddylated SCF intact. The SCF is now subject to auto-ubiquitination and subsequent degradation which leads to accumulation of SCF substrates in yeast and Neurospora (He et al., 2005; Wee et al., 2005). This shows that the neddylation and deneddylation of cullins is a highly dynamic and important process, on the other hand misregulation of substrate degradation leads to severe consequences for a living organism.

Interestingly the CSN does not only show deneddylation activity but it also displays a ubiqitin isopeptidase activity. The CSN can through the metalloprotease domain of CSN5 either depolymerize ubiquitin chains or de-ubiquitinate mono-ubiquitinated substrates, which suggests that the mechanisms of deneddylation and de-ubiquitination are similar from fission yeast to human (Groisman et al., 2003; Zhou et al., 2003).

Another important feature of the CSN are associated kinase activities. To date three associated kinase activities have been found. The first identified kinase is a inositol 1,3,4triphosphate 5/6-kinase or short 5/6-kinase of Arabidopsis (Wilson et al., 2001). It was shown that the 5/6-kinase physically interacts with the CSN1 subunit of the CSN and overexpression of CSN1 was shown to repress this kinase activity (Wilson et al., 2001). Further on in HeLa cells the kinases CK2 and PKD were found associated with the CSN and able to phosphorylate subunits of the CSN and c-Jun and p53, thus directly regulating the ubiquitin conjugation of these transcription factors (Uhle et al., 2003). All three kinases were found to be inhibited by curcumin (Sun et al., 2002; Uhle et al., 2003; Wilson et al., 2001). Phosphorylation of c-Jun, a part of the AP-1 transcription complex, leads to stabilisation of the protein in proliferating cells (Dunn et al., 2002), the phosphorylation of the tumor suppressor p53 leads to destabilisation of the protein (Sharpless and DePinho, 2002), and disruption of the CSN leads to accumulation of p53 and eventually to cell cycle arrest and cell death (Bech-Otschir et al., 2001). 


\subsubsection{The role of $\mathrm{CSN}$ in control of cellular functions}

The CSN is a global regulator of development in higher and lower eukaryotes. Its effect on development and physiology has been studied in depth in recent years in numerous organisms like mammals, worm and fly, plant and in fungi (Busch et al., 2003; Freilich et al., 1999; Mundt et al., 2002; Serino and Deng, 2003; Wee et al., 2002; Yan et al., 2003; Zhou et al., 2001). Generally, the role of the CSN is diverse in these organisms, it reaches from lightrelated signal transduction (He et al., 2005; Wei and Deng, 1992; Wei et al., 1994), oogenesis (Doronkin et al., 2003), immune response (Boussiotis et al., 2000; Kleemann et al., 2000), apoptosis (Yan et al., 2003), cell cycle control (Mundt et al., 1999), checkpoint control (Liu et al., 2003) to DNA repair (Groisman et al., 2003).

The cellular function, development and maintenance of (multi-)cellular organisms strongly relies on the proper complexation of the eight subunits of the CSN. A loss of single subunits of the CSN leads to a loss of the whole complex and thus severe cellular and developmental defects or even cell death (Busch et al., 2003; Freilich et al., 1999; Oron et al., 2002; Smith et al., 2002; Tomoda et al., 2004).

In mammals embryonic development relies, among other factors, on the regulation of cyclin $\mathrm{E}$ and p53 protein stability by the COP9 signalosome through the SCF. Misregulation or loss of Uba3 (Tateishi et al., 2001) and Cul1 (Dealy et al., 1999), as parts of the neddylation machinery and SCF, or the loss of single subunits of the CSN leads to a stabilisation of p53 following apoptosis and on the other hand to dysregulation of cyclin E (Lykke-Andersen et al., 2003; Wang et al., 1999). Cyclin E in complex with Cdk2 mediates phosphorylation and subsequent ubiquitination and degradation of the Cdk inhibitor p27 during late G1 impeding progression to S phase (Sherr and Roberts, 1999; Slingerland and Pagano, 2000). The precise mechanism of p27 regulation during development through the CSN remains to be fully uncovered, though. In human the Smith-Magenis Syndrom (SMS) has been assigned to a 1,52,0MB hemizygous deletion on chromosome 17, containing approx. 20 genes including CSN3 (Elsea et al., 1999; Potocki et al., 1999; Potocki et al., 2000). Patients suffering from SMS display genital anomalies and mental retardation in addition to a phase shift of circadian rhythm leading to severe sleep disturbances (De Leersnyder et al., 2001; Greenberg et al., 1996).

CSN has essential functions in Drosophila oogenesis and embryogenesis, whereas csn mutants are lethal at early larval stages (Freilich et al., 1999; Oron et al., 2002). Mutation analyses of the gene for the CSN5 subunit have shown that the CSN is needed for 
photoreceptor $\mathrm{R}$ cell differentiation and promotion of laminal glial cell migration and axon targeting, indicating that deneddylation is needed during development (Suh et al., 2002) (Cope et al., 2002). Eggs of Drosophila csn mutants display characteristic disruptions in anteroposterior and dorsoventral axis formation due to activation of a DNA damage checkpoint (Doronkin et al., 2002). In C. elegans knockdowns of CSN5 by RNAi result in a sterile phenotype with small gonads and no oocytes (Smith et al., 2002).

In Arabidopsis, csn mutants survive embryogenesis, but die after germination at seedling stage with characteristic phenotypes: the mutants are small and accumulate high levels of the anthocyanin pigment. They display a constitutive light-morphology with a gene expression profile similar to that of seedlings under high-light intense stress (Ma et al., 2003). Csn mutants with decreased expression of single subunits on the other hand are viable but display various abnormalities in adult plants (Peng et al., 2001a, b; Schwechheimer et al., 2002; Wang et al., 2002). Some of the development defects occur because of mismodulation of SCF E3 ligases by the CSN. It could be shown, that a direct interaction of CSN with SCF ${ }^{\mathrm{TIR} 1}$ influences the activity of this specific SCF complex in mediating auxin response, hindering $\mathrm{SCF}^{\mathrm{TIR} 1}$ from degrading target proteins and abolishing the auxin response (Schwechheimer et al., 2002). The $\mathrm{CSN}$ was also found to interact directly with $\mathrm{SCF}^{\mathrm{UFO}}$ regulating $\mathrm{SCF}^{\mathrm{UFO}}$ mediated flower development (Wang et al., 2003). In a third case the CSN was found to interact with $\mathrm{SCF}^{\mathrm{COI} 1}$ regulating the plant defense-response (Feng et al., 2003). The CSN seems to modulate a variety of SCF activities in Arabidopsis in diverse development pathways.

In several fungal species the cellular role of the CSN has been investigated. In contrast to higher eukaryotes, fungal mutants with mutations in one or more genes for the subunits of the CSN are viable. In S. pombe mutations in the genes for subunits CSN1 and CSN2 exhibit an elongated cell phenotype, slow growth and sensitivity to UV and gamma irradiation (Liu et al., 2003). The reason for this is that Csn 1 and Csn2 are required to regulate ribonucleotide reductase (RNR) through the degradation of a small protein, Spd1, that acts to anchor the small RNR subunit in the nucleus. Spd1 destruction correlates with the nuclear export of the small RNR subunit, which, in turn, correlates with a requirement for RNR in replication and repair (Liu et al., 2003). Mutations in the genes for subunits CSN3-5 do not produce any phenotypes, but the mutants are not capable of deneddylation (Mundt et al., 2002; Zhou et al., 2001). In S. cerevisiae COP9 mutants display enhanced pheromone response and increased mating efficiency (Maytal-Kivity et al., 2002a). Recently, He et al. discovered that in $N$. crassa the COP9 signalosome regulates the circadian clock by controlling the stability of the 
$\mathrm{SCF}^{\mathrm{FWD}-1}$ complex. FWD-1 is an F-box protein that is specific for FREQUENCY (FRQ) degradation, a circadian clock protein that is critical for clock function. A mutation in $\operatorname{csn}-2$ leads to degradation of $\mathrm{SCF}^{\mathrm{FWD}-1}$ and stabilisation of FRQ, thus abolishing a natural lightdark dependent conidiation rhythm. Another example of developmental regulation by CSN is the fungus Aspergillus nidulans. Csn deletion strains of A. nidulans are viable but strains are impaired in cell polarity and accumulate a red pigment (Busch et al., 2003). Furtheron the mutants enter the sexual cycle and form fruitbodies that are blocked in development at the stage of primordia formation. The development and maturation from primordia to fertile cleistothecia does not take place (Busch et al., 2003).

\subsection{Control of amino acid biosynthesis in fungi}

\subsubsection{The transcriptional activator of the general/cross-pathway control}

Amino acids are important building blocks for protein production in the cell. A rapid response to nutritional and other external stimuli is of great importance for the cell to adopt and allow transcriptional reprogramming in case of a lack of amino acids or carbon or nitrogen sources (Davis et al., 2005). Most fungi prefer to take up amino acid from their diet, but are able to produce amino acids themselves in times of hardship. When amino acids are present in the medium, no further specific enzyme activities are needed. On the other hand under starvation conditions counter-actions of the cell need to be imposed and orchestrated. In case of starvation on one or more amino acids the biosynthesis of all amino acids is turned on. To regulate amino acid biosynthesis a transcription factor regulates transcription in the cells. This control network is called general control (gc) of amino acid biosynthesis in S. cerevisiae or cross-pathway control (cpc) in filamentous fungi (for an overview see Figure 4) (Hinnebusch, 1986; Sachs, 1996). Gcn4p of S. cerevisiae or CpcA of A. nidulans and CPC-1 of N. crassa are the central regulatory transcription factors of these pathways (Braus et al., 2004; Hinnebusch and Natarajan, 2002). The C-terminus of the proteins consists of a basic leucine zipper, commonly seen in transcription factors of the AP-1 family of basic leucine zippers (bZIP) (Kouzarides and Ziff, 1989). These approximately 60 amino acids are sufficient for homo-dimerization and binding to the DNA (Hope and Struhl, 1986). Other transcription factors of the AP-1 family like c-jun and c-Fos are in contrast to fungal members of this family not restricted to homo-dimerization (Turner and Tjian, 1989). Two nuclear localisation 
signals (NLS) have been found for Gcn4p, whereas A. nidulans CpcA only contains one NLS in the C-terminal region, overlapping some amino acids of the binding function (Pries et al., 2004). The NLS signal is essential for translocation of the transcription factor into the nucleus independent of amino acid availability (Pries et al., 2002). The activation domains, needed for efficient stimulation of transcription, of the transcription factors of the gc/cpc are not was well conserved as the dimerization domains. In yeast this activation domain (AD) is divided further in two subdomains, the central acidic activation domain (CAAD) and the N-terminal activation domain (NTAD), this concept of divided activation domains seems to be conserved among other fungi (Drysdale et al., 1995). In yeast the two activation domains are divided by a characteristic PEST region, named after their amino acid content (Rechsteiner and Rogers, 1996). This PEST region harbors phosphorylation and ubiquitination sites responsible for the instability of the protein which is finally degraded in the 26S proteasome (Kornitzer et al., 1994). Under starvation conditions the transcription factor binds to defined nucleotide sequences in the promoter regions of amino acid biosynthesis genes and increases the transcription of those genes. This specific sequence is called Gcn $4 p$ protein response element (GCRE) in yeast or CpcA protein response element (CPRE) in filamentous fungi, but is also found as a binding site for mammalian AP-1 counterparts (Arndt and Fink, 1986; Hoffmann et al., 2001; Hope and Struhl, 1985; John et al., 1996; Oliphant et al., 1989). The sequence of the binding site is palindromic and contains $9 \mathrm{bp}$ of the following sequence: $5^{\prime}-\mathrm{ATGA}(\mathrm{C} / \mathrm{G}) \mathrm{TCAT}^{-} 3^{\prime}$. It is found upstream of the promoters of numerous target proteins and upstream of the promoters of $c p c-1$ and $c p c A$, where under starvation conditions transcriptional auto-regulation takes place (Ebbole et al., 1991; Hoffmann et al., 2001). The transcription factors Gcn $4 \mathrm{p}, \mathrm{CpcA}$ and $\mathrm{CPC}-1$ are fully interchangeable, indicating a highly conserved mechanism for gc/cpc between yeast and filamentous fungi (Hoffmann et al., 2001). 


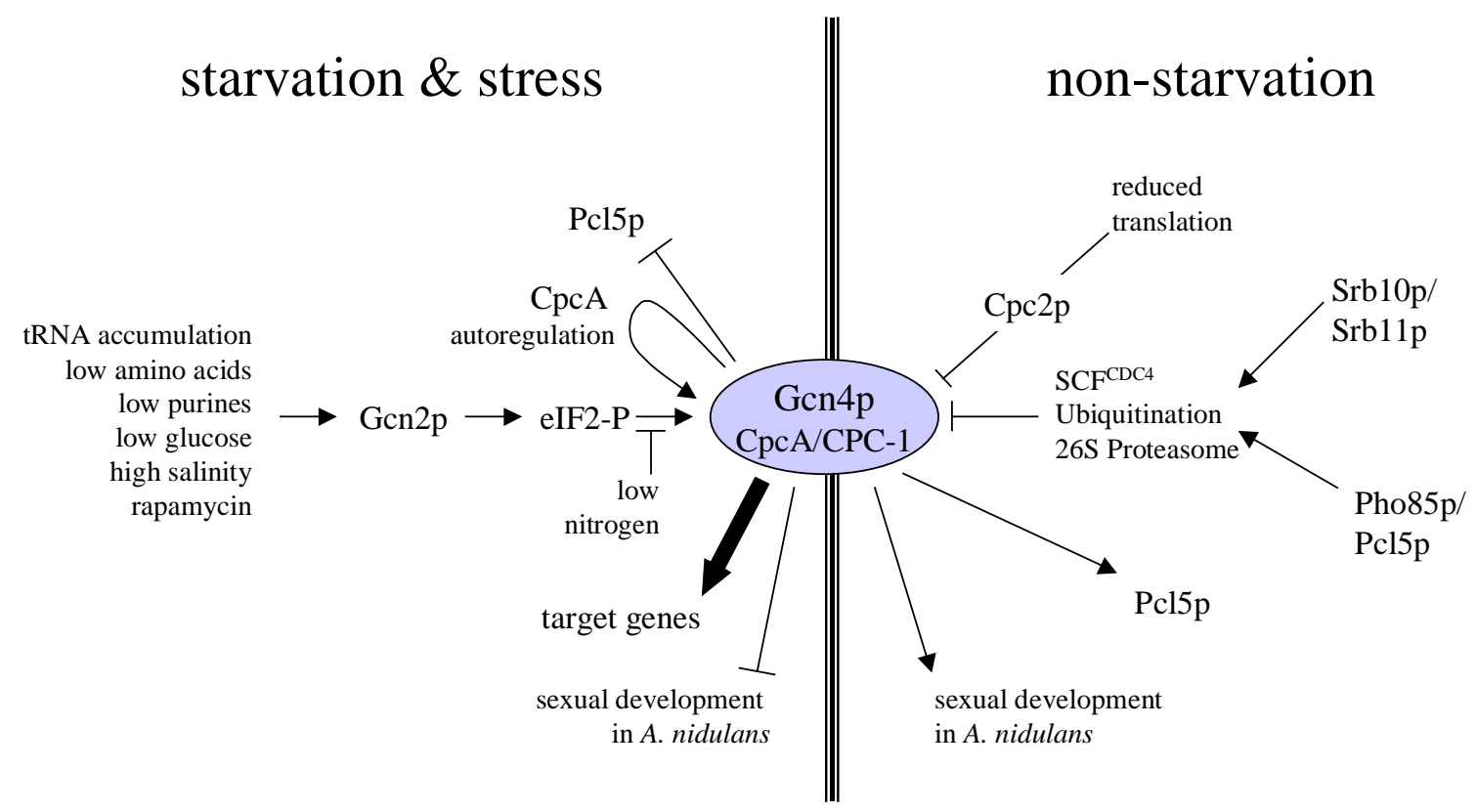

Fig. 4: Summarizing overview of the general control/cross-pathway control of fungi

The complex network induced by Gcn $4 \mathrm{p}$ and presumably its counterparts in other fungi is displayed according to Hinnebusch and Natarajan, 2002; Irniger and Braus, 2003; Shemer et a.l, 2002; Grundmann et al., 2001; Pries et al., 2002; Valerius et al., 2001; Ebbole et al., 1991 and Hoffmann et al., 2000/2001. Details see text. 


\subsubsection{Translational control of Gcn4p synthesis in $S$. cerevisiae}

Due to the fact that Gcn4p is a global transcription factor regulating diverse cellular pathways its activity needs to be strongly regulated. In the case of $S$. cerevisiae this happens on translational as well as post-translational levels through degradation of the protein (Braus et $a l ., 2004)$. Cells need to decide or sense whether amino acids are present or not, then decide whether the biosynthesis of amino acids needs to be switched on or not. If the fungal cell senses a lack of one or more amino acids, the biosynthesis of amino acids is globally turned on in contrast to bacteria where only the biosynthesis of the respective amino $\operatorname{acid}(\mathrm{s})$ is activated (Cahel and Rudd, 1987). To sense the presence of amino acids S. cerevisiae, N. crassa and Aspergillus (see Chapter 3) have amino acid sensorkinases, called Gcn2p or CPC-3 or CpcC, respectively (Carsiotis and Jones, 1974; Carsiotis et al., 1974; Piotrowska et al., 1980). The sensor is combined with a kinase and is located in the cytoplasm attached to the ribosome (Dong et al., 2000). The sensor kinase monitors the availability of amino acid charged tRNAs and regulates the overall translation performance.

Gcn2p/CPC-3 consists of a C-terminal histidyl-tRNA synthetase (HisRS)-related domain and a N-terminal protein kinase domain (Harashima and Hinnebusch, 1986; Zhu et al., 1996). The HisRS kinase responds to uncharged tRNAs by activating the N-terminal protein kinase activity (Dong et al., 2000). The tRNA-binding domain of Gcn2p also contains a dimerization domain that interacts with another monomer, the kinase domain and is needed for kinase activation (Qiu et al., 2001; Wek et al., 1995). In vivo the sensor kinase needs to be complexed to Gen20p/Gen1p which bind to the N-terminal domain of Gen2p to function (Garcia-Barrio et al., 2000; Kubota et al., 2000; Kubota et al., 2001). Gcn1p seems to bind near the ribosomal entry site for aminoacyl-tRNAs and promotes Gcn2p kinase activity when uncharged tRNAs bind to the entry site and to Gcn2p (Sattlegger and Hinnebusch, 2000). This leads to activation of the kinase domain of Gcn2p. The kinase then phosphorylates the $\alpha$ subunit of eukaryotic elongation initiation factor eIF-2 resulting in a block of the elongation factor and hence a general repression of translation (Hinnebusch and Natarajan, 2002).

Gen4p expression is under slight transcriptional control and translated on a basal, low level even under non-starvation conditions (Albrecht et al., 1998). The promoter of GCN4, cpc-1 and $c p c A$ contains small open reading frames (uORFs). Four uORFs are found in yeast and two slightly larger ones in N. crassas and A. nidulans 5'-leader region of the promoter, respectively. Under starvation conditions, where translation in general is repressed, these uORFs allow for efficient translation of the open reading frames of the transcription factors, 
whereas they repress translation effectively under non-limiting conditions (Hinnebusch and Natarajan, 2002). At the onset of translation initiation the small ribosomal subunit binds to the $5^{\prime}$-end of the GCN4/cpc-1/cpcA-mRNA and forms an initiation complex with eIF-2 $\alpha$-GTP and the initiation methionine-tRNA at the first AUG START codon that is reached while scanning the mRNA. During initiation of translation the GTP of eIF-2 $\alpha$ is hydrolized to GDP. After reaching the STOP codon the ribosome dissociates from the mRNA, leaving the small ribosomal subunit attached. For the next initiation the GDP needs to be replaced by GTP by the GEF. Under non-limiting conditions this takes place prior to the AUG START codon of the fourth uORF, which is subsequently translated and the ribosome dissociates without being able to recharge eIF-2 $\alpha$ with GTP and thus translation of the coding sequence of $G C N 4 / c p c$ $1 / c p c A$ is repressed. Under amino acid limiting conditions the phosphorylation of eIF-2 $\alpha$ inhibits the GEF eIF2B, thus the recycling rate of the translation initiation complex is slowed down. In this case the first $\mathrm{UORF}$ is translated, but re-initiation of translation does not take place prior to the fourth uORF, but before the coding sequence of the mRNA leading to increased expression of the transcription factors (Hinnebusch, 1997). For N. crassa CPC-1 and $A$. nidulans $\mathrm{CpcA}$ this means that they can increase their transcription through the CPREs in their respective promoter regions, thus increasing their own production even stronger (Hoffmann et al., 2001). Under nitrogen starvation conditions induction of GCN4 mRNA translation by amino acid analogues can be overruled in yeast by an additional repression mechanism independent of eIF-2 $\alpha$ phosphorylation by Gcn2p (Grundmann et al., 2001).

The ribosome bound WD-repeat proteins $\mathrm{Cpc} 2 \mathrm{p} / \mathrm{CpcB} / \mathrm{CPC}-2$ incorporate another regulatory mechanism to the expression of the transcription factors. In $c p c 2$ mutants the transcription of GCN4 target genes is increased but not the protein level, suggesting no specific role in GCN4 mRNA translation (Hoffmann et al., 1999). Deletions in CPC2 lead to increased Gcn4p dependent transcriptional activation of its target genes under non-starvation conditions in wild type cells and under non-starvation and starvation conditions in gcn2 mutants (Hoffmann et al., 1999). The full cellular function of the protein remains yet unclear, but it was hypothesized that $\mathrm{Cpc} 2 \mathrm{p}$ is activated on ribosomes translating at the maximum rate in cells growing and is then deactivated by Gen2p in starved cells (Hinnebusch and Natarajan, 2002). The corresponding rat protein RACK-1 is an intracellular receptor for activated protein kinase C (PKC) involved in the cellular localisation of PKC (Rotenberg and Sun, 1998) and expression of the mammalian protein complements the general control defect in yeast $c p c 2$ mutants (Hoffmann et al., 1999). 
The yeast TOR (target of rapamycin) proteins have a moderate positive impact on the translation of Gen4p. It was shown with GCN4 reporter constructs that rapamycin slightly induced GCN4 translation (Valenzuela et al., 2001). The eIF2a kinase Gcn2p activity is regulated by phosphorylation by the TOR kinase and dephosphorylated by Tap42p under amino acid starvation and non-starvation conditions respectively (Cherkasova and Hinnebusch, 2003). It is speculated that the increased expression of Gcn $4 p$ target genes required for acquisition of less-favoured nitrogen sources bases most likely to the fact that a slight starvation on nitrogen leads to a slight starvation on amino acids (Valenzuela et al., 2001).

\subsubsection{Post-translational control of Gcn4p expression}

After synthesis of the transcription factors $\mathrm{Gcn} 4 \mathrm{p} / \mathrm{CCP}-1 / \mathrm{CpcA}$ in the cytosol these need to be transported into the nucleus to increase transcription of their target genes. Their cellular destination is marked by their nuclear localisation sequences (Pries et al., 2002). Since the cytosol and the nucleus are divided by the nuclear membrane, the proteins need to be channeled through nuclear pores (Lusk et al., 2004). This process is driven by GTP hydrolysation by the small GTPase Ran, though the initial step of nuclear import is carried out by the formation of a heterodimeric importin $\alpha / \beta$ complex in the cytoplasm which recognizes the NLS as part of the cargo protein, translocates the cargo into the nucleus and returns to the cytoplasm (Lusk et al., 2004). The importins necessary to import Gcn4p into the nucleus were identified to be the $\alpha$-importin Srp1p and the $\beta$-importin Kap95p (Pries et al., 2004). Import of the transcription factor Gcn $4 p$ seems to be constitutive and independent of the nutritional status (Pries et al., 2004).

Once Gcn $4 p$ has entered the nucleus it is subject to phosphorylation through cyclin dependent kinases (CDK) and subsequent degradation in the 26S proteasome (Pries et al., 2002). To date nothing is known about the stability of Gcn $4 p$ counterparts in filamentous fungi or if there is a regulation of the stability of $\mathrm{CPC}-1$ or $\mathrm{CpcA}$ at all. In $S$. cerevisiae stability of $\mathrm{Gcn} 4 \mathrm{p}$ is highly regulated (Irniger and Braus, 2003): under non-limiting conditions the protein is unstable with a half-life of approximately 5 minutes. Under starvation conditions the half-life of the protein increases to approximately 20 minutes (Kornitzer et al., 1994). Cyclins are unstable proteins and are known for their role in various steps of the cell division cycle. The cyclin Pcl5p is known to take part in the degradation of Gcn4p. By assembly of Pcl5p with its 
cyclin CDK (cyclin dependent kinase) Pho85p Gcn4p is phosphorylated at Thr165 which leads to ubiquitination and subsequent degradation in the $26 \mathrm{~S}$ proteasome (Shemer et al., 2002). Under starvation conditions the instable Pcl5p is not found throughout the cell, most likely due to its rapid turnover which can not be counteracted by transcriptional activation through Gen $4 p$ (Jia et al., 2000).

The second important CDK known to destabilize Gen4p is Srb10p. Srb10p seems to constitutively destablize Gcn $4 p$. The CDK is part of the mediator, a multiprotein complex which is part of the RNA polymerase holoenzyme II and is needed to mediate between transcription factors and the RNA polymerase II (Lusk et al., 2004). Thus Srb10p may be involved in limiting the transcripts (and resulting proteins) for bound Gen $4 p$ at its target promoters enabling a fast turn-down of the gc/cpc if necessary (Chi et al., 2001).

By phosphorylation at residue Thr165 through the CDKs Gen4p is target to ubiquitination through the SCF (see chapter 3), where Cdc4p is the specificity protein for Gcn $4 p$ ubiquitination (Meimoun et al., 2000). Ubiquitination of Gcn4p results in its rapid degradation in the $26 \mathrm{~S}$ proteasome.

\subsubsection{Gc/cpc dependent activation of transcription in fungi}

Transcriptional profiling in S. cerevisiae showed that at least 539 genes of the genome were targets of Gen $4 p$ under starvation conditions (Natarajan et al., 2001). The target genes encompassed encoded not only for amino acid biosynthesis proteins, but also for vitamin biosynthesis enzymes, peroxisomal components, mitochondrial carrier proteins and also autophagy proteins and other transcription factors (Natarajan et al., 2001). This analysis showed a wide range of pathways regulated by Gcn4p, making Gen $4 p$ a master regulator of cellular functions. Interestingly Gen4p seems also important for a general stress response like purine or glucose starvation, salt stress or UV light (Engelberg et al., 1994; Goossens et al., 2001; Natarajan et al., 2001; Rolfes and Hinnebusch, 1993) and cellular adhesion in diploid $S$. cerevisiae strains (Kleinschmidt et al., 2005).

Interestingly there seem to be three major mechanisms how Gcn $4 p$ activates the transcription of certain sets of genes - reorganisation of chromatin structure, histone modification and recruitment of the transcription machinery (Drysdale et al., 1998; Natarajan et al., 1999).

One function of the Gen4p activation domain seems to loosen the chromatin structure of promoters and enable transcription. The smallest unit of chromatin is a nucleosome, 
consisting of an octamer of the histones H2A, H2B, H3 and H4 wrapped with 146bp of DNA (Luger et al., 1997; Noll and Kornberg, 1977; Shaw et al., 1976). In this structure the DNA is protected against the access of transcription factors resulting in repressed gene expression (Kornberg and Lorch, 1991). Usually the promoter region comprising the TATA element where the transcription factor complex TFIID binds is masked by chromatin. Activator derepression weakens the DNA-histone interactions and enables transcription factors to access the promoters. The transcription activators do not have the immediate ability to de-repress the histone-bound promoters, but rather recruit other chromatin remodeling or modifying complexes in order to activate their target genes.

The eukaryotic SWI2/SNF2 complex is a well-characterized model of chromatin remodelers. Chromatin remodeling requires ATP and sequence similarities between the energy-consuming ATPase subunits which are characteristic for this protein family. The SWI2/SNF2 complex is recruited to target loci and binds independent of any sequence specifity to DNA and nucleosomes (Boeger et al., 2005; Cote et al., 1998; Quinn et al., 1996). The amount of $S$. cerevisiaes SWI2/SNF2 complex is very low and it needs to be recruited specifically to the respective target loci, where it disrupts the nucleosomes and gives the transcription factor access to the now prone promoter region. It is shown for HIS3 that a physical interaction of SWI2/SNF2 and Gcn $4 \mathrm{p}$ is required for efficient transcription activation by Gcn $4 \mathrm{p}$ (Holstege et al., 1998; Natarajan et al., 1999), though only a subset of all genes requires the activity of SWI2/SNF2 for transcription activation (Holstege et al., 1998). The hydrophobic clusters of the Gcn $4 p$ activation domain are important for the interaction with the SWI2/SNF2 complex (Neely et al., 2002). Therefore, one way of transcription activation by Gen4p is to bind to nucleosomal DNA and recruit SWI2/SNF to specific promoter regions.

Gen $4 p$ is also able to affect histone modification and thus alteration of chromatin structure to access promoter regions of some genes. Several reversible covalent modifications are known so far that affect the local chromatin structure (Ito, 2003; Verger and Crossley, 2004). The best studied example of chromatin modification is acetylation/deacetylation of histone subunits, mainly $\mathrm{H} 3$ and $\mathrm{H} 4$ and to a lesser extend the H2 histones (Khan and Krishnamurthy, 2005; Khochbin and Kao, 2001). In fungi Gcn5p is well characterized as a histone acetylase which acetylates $\mathrm{H} 3$ and H4. For HIS3 it was shown that Gcn4p dependent transcription relies on Gcn5p dependent H3 acetylation (Filetici et al., 1998). Gcn5p expresses its acetylase activity as part of the SAGA complex ( $\underline{\text { ppt- }}$ Ada- $\underline{G}$ n5 acetyl transferase)(Roberts and Winston, 1997; Timmers and Tora, 2005). As for the SWI2/SNF2 complex SAGA needs to be recruited to the respective promoter regions, where in the case of amino acid biosynthesis 
control the transcription activator Gcn $4 p$ recruits the complex with the hydrophobic part of its activation domain (Drysdale et al., 1998).

A third mechanism of Gen $4 p$ activating transcription of target genes is direct interaction with the transcription machinery. Gcn4p can bind directly to TFIID which binds to the TATA box. TFIID consists of the actual TATA binding protein (TBP) and several TBP-associated factors (TAFs) with multiple functions in the transcription initiation complex (Green, 2000; Matangkasombut et al., 2004). The interaction of TFIID and Gcn4p is mediated by the multiprotein bridging factor Mbf1p, which is highly conserved from yeast to man (Kabe et al., 1999; Takemaru et al., 1998). Mbf1p seems to bind to the DNA-binding moiety of Gcn4p rather than to the activation domain, indicating that the actual binding of the transcription factor can promote trans-activation (Takemaru et al., 1998). In addition Gen4p can directly recruit the RNA polymerase II holoenzyme, which is important for the actual transcription of the target genes, via the mediator complex (MED) (Kim et al., 1994). The co-activator MED consists of more than 20 proteins, one of which is Srb10p (see above) (Qiu et al., 2004), and interacts transcription factors with RNA polymerase II subunits.

\subsubsection{Impact of the cross-pathway control on $A$. nidulans development}

In filamentous fungi the lack of translational precursors has drastic effects on the development of these organisms. The absence of amino acid and hence an active $\mathrm{gc} / \mathrm{cpc}$ results in a strong impact on A. nidulans developmental program. Strains auxotrophic for amino acids are dependent on a suitable supply of amino acids in their medium. Auxotrophic strains defective in the tryptophan pathway are blocked in the formation of conidia and cleistothecia (Eckert et al., 1999; Käfer, 1977; Yelton et al., 1983). A systematic study on four different tryptophan biosynthesis genes showed that fruitbody formation could be restored by high concentrations of tryptophan and was promoted by the addition of indole or auxin, whereas the fertility of sexual fruitbodies could only be partially restored. An increasing amount of tryptophan is necessary to pass the three major steps of development of A. nidulans: mycelia growth, conidiation and formation of cleistothecia (Eckert et al., 1999). Deletion in the $\operatorname{trp} B$ gene showed that these effects are directly linked to the loss of activity itself, similar results were found for the effects of the loss of the hisB gene (Busch et al., 2001; Eckert et al., 1999). $\operatorname{argB}$ mutant strains are deficient in the formation of cleistothecia and on the other hand excessive supply of arginine inhibits ascospore formation in the wild type (Serlupi-Crescenzi et al., 1983). 
Auxotrophic A. nidulans strains are blocked in fruitbody formation and amino acid limitation results in impaired fruitbody formation (Eckert et al., 1999; Eckert et al., 2000). A. nidulans strains growing under amino acid starvation conditions are able to initiate the formation of cleistothecia, but are blocked at the stage of microcleistothecia before the completion of meiosis. The resulting microcleistothecia are filled with hyphae and considerably smaller than cleistothecia. This block in cleistothecia formation can be overcome by addition of the respective amino acids to the medium (Hoffmann et al., 2000).

An artificially simulated amino acid starvation due to overexpression of CpcA as well as the yeast counterpart Gcn $4 p$ results in the same block even in the absence of amino acid limitation, suggesting that gc/cpc related signals interfere with the developmental program (Hoffmann et al., 2000). The deletion of $c p c B$, which presumably acts as an inhibitor of the $\mathrm{gc/cpc}$ under non-limiting conditions, also results in the formation of microcleistothecia (Hoffmann et al., 2000).

Concluding one could assume that the block of sexual development in A. nidulans seems to be an economical consequence of a lack of building material in nature, mediated by elevated expression of the transcription factor CpcA.

\subsection{Scope and aim of this work}

Well studied examples of biological and biotechnological relevant organisms are species of the gender Aspergillus. Aspergilli are filamentous ascomycetes. So far more than 185 Aspergilli are known. At least 20 Aspergilli are human pathogen. Aspergillus fumigatus and Aspergillus flavus produce $\beta$-Lactam antibiotics and aflatoxin. Aspergillus fumigatus has become the most relevant human pathogen causing invasive pulmonary aspergillosis in immunocompromised patients. Aspergillus oryzae is of high biotechnological importance in asian countries, being used in the production of soy sauce, sake and miso. Aspergillus nidulans is capable of very complex and diverse biosynthesis and differentiation processes where after mating with a compatible partner or "selfing" cleistothecia are formed which contain octades of ascospores. These examples of the Aspergillus family are relatively easy genetically manipulated and thus provide suitable model organisms for studying regulatory networks of molecular cross-pathway connections between environmental stimuli, metabolism and development. 
This study directs the focus on two possibly interacting regulatory networks: the COP9 signalosome, important for development of the fungus Aspergillus nidulans, and the crosspathway control network necessary for amino acid biosynthesis. Chapter 2 focusses on CsnA which is the largest and therefore first subunit of the COP9 signalosome of A. nidulans. The effects of loss of function and partial expression of the gene on development of the fungus are analysed and single protein functions are separated from COP9 signalosome functions. Chapter 3 shows an in depth investigation on cross-pathway control related genes and proteins in all three above mentioned Aspergilli, based on the recently released genome sequences of the ascomycete A. nidulans and the deuteromycetes A. fumigatus and A. oryzae. In chapter 4 an analysis of the cross-pathway control transcription factor CpcA is provided, focussing on nuclear import of the protein into the nucleus. 


\subsection{References}

Albrecht, G., Mösch, H.U., Hoffmann, B., Reusser, U., and Braus, G.H. (1998) Monitoring the Gcn4 proteinmediated response in the yeast Saccharomyces cerevisiae. J Biol Chem 273: 12696-12702.

Ambroggio, X.I., Rees, D.C., and Deshaies, R.J. (2004) JAMM: A Metalloprotease-Like Zinc Site in the Proteasome and Signalosome. PLoS Biol 2: E2.

Arndt, K., and Fink, G.R. (1986) GCN4 protein, a positive transcription factor in yeast, binds general control promoters at all 5' TGACTC 3' sequences. Proc Natl Acad Sci U S A 83: 8516-8520.

Bech-Otschir, D., Kraft, R., Huang, X., Henklein, P., Kapelari, B., Pollmann, C., and Dubiel, W. (2001) COP9 signalosome-specific phosphorylation targets p53 to degradation by the ubiquitin system. EMBO J 20: 1630-1639.

Bech-Otschir, D., Seeger, M., and Dubiel, W. (2002) The COP9 signalosome: at the interface between signal transduction and ubiquitin-dependent proteolysis. J Cell Sci 115: 467-473.

Berndt, C., Bech-Otschir, D., Dubiel, W., and Seeger, M. (2002) Ubiquitin system: JAMMing in the name of the lid. Curr Biol 12: R815-817.

Boeger, H., Bushnell, D.A., Davis, R., Griesenbeck, J., Lorch, Y., Strattan, J.S., Westover, K.D., and Kornberg, R.D. (2005) Structural basis of eukaryotic gene transcription. FEBS Lett 579: 899-903.

Boussiotis, V.A., Freeman, G.J., Taylor, P.A., Berezovskaya, A., Grass, I., Blazar, B.R., and Nadler, L.M. (2000) p27kip1 functions as an anergy factor inhibiting interleukin 2 transcription and clonal expansion of alloreactive human and mouse helper T lymphocytes. Nat Med 6: 290-297.

Braus, G.H., Pries, R., Düvel, K., and Valerius, O. (2004) Molecular biology of fungal amino acid biosynthesis regulation. In The Mycota II. Kück, U. (ed). Berlin-Heidelberg: Springer-Verlag, pp. 239-269.

Bucher, P., Karplus, K., Moeri, N., and Hofmann, K. (1996) A flexible motif search technique based on generalized profiles. Comput Chem 20: 3-23.

Busch, S., Hoffmann, B., Valerius, O., Starke, K., Düvel, K., and Braus, G.H. (2001) Regulation of the Aspergillus nidulans hisB gene by histidine starvation. Curr Genet 38: 314-322.

Busch, S., Eckert, S.E., Krappmann, S., and Braus, G.H. (2003) The COP9 signalosome is an essential regulator of development in the filamentous fungus Aspergillus nidulans. Mol Microbiol 49: 717-730.

Cahel, M., and Rudd, K.E. (1987) The stringent response. In Escherichia coli and Salmonella Typhimurium: cellular and molecular biology. Neidhardt, F.C., Ingraham, J.L., Low, K.B., NMagasanik, B., Schaechter, M. and Umbareger, H.E. (eds). Washington, DC: American Society for Microbiology, pp. 1410-1438.

Carrabino, S., Carminati, E., Talarico, D., Pardi, R., and Bianchi, E. (2004) Expression pattern of the JAB1/CSN5 gene during murine embryogenesis: colocalization with NEDD8. Gene Expr Patterns 4: $423-431$.

Carsiotis, M., and Jones, R.F. (1974) Cross-pathway regulation: tryptophan-mediated control of histidine and arginine biosynthetic enzymes in Neurospora crassa. J Bacteriol 119: 889-892. 
Carsiotis, M., Jones, R.F., and Wesseling, A.C. (1974) Cross-pathway regulation: histidine-mediated control of histidine, tryptophan, and arginine biosynthetic enzymes in Neurospora crassa. J Bacteriol 119: 893-898.

Chamovitz, D.A., and Segal, D. (2001) JAB1/CSN5 and the COP9 signalosome. A complex situation. EMBO Rep 2: 96-101.

Cherkasova, V.A., and Hinnebusch, A.G. (2003) Translational control by TOR and TAP42 through dephosphorylation of eIF2alpha kinase GCN2. Genes Dev 17: 859-872.

Chi, Y., Huddleston, M.J., Zhang, X., Young, R.A., Annan, R.S., Carr, S.A., and Deshaies, R.J. (2001) Negative regulation of Gen4 and Msn2 transcription factors by Srb10 cyclin-dependent kinase. Genes Dev 15 : 1078-1092.

Cope, G.A., Suh, G.S., Aravind, L., Schwarz, S.E., Zipursky, S.L., Koonin, E.V., and Deshaies, R.J. (2002) Role of predicted metalloprotease motif of Jab1/Csn5 in cleavage of Nedd8 from Cul1. Science 298: 608-611.

Cope, G.A., and Deshaies, R.J. (2003) COP9 signalosome: a multifunctional regulator of SCF and other cullinbased ubiquitin ligases. Cell 114: 663-671.

Cote, J., Peterson, C.L., and Workman, J.L. (1998) Perturbation of nucleosome core structure by the SWI/SNF complex persists after its detachment, enhancing subsequent transcription factor binding. Proc Natl Acad Sci U S A 95: 4947-4952.

Davis, M.A., Askin, M.C., and Hynes, M.J. (2005) Amino acid catabolism by an areA-regulated gene encoding an L-amino acid oxidase with broad substrate specificity in Aspergillus nidulans. Appl Environ Microbiol 71: $3551-3555$

De Leersnyder, H., De Blois, M.C., Claustrat, B., Romana, S., Albrecht, U., Von Kleist-Retzow, J.C., Delobel, B., Viot, G., Lyonnet, S., Vekemans, M., and Munnich, A. (2001) Inversion of the circadian rhythm of melatonin in the Smith-Magenis syndrome. J Pediatr 139: 111-116.

Dealy, M.J., Nguyen, K.V., Lo, J., Gstaiger, M., Krek, W., Elson, D., Arbeit, J., Kipreos, E.T., and Johnson, R.S. (1999) Loss of Cul1 results in early embryonic lethality and dysregulation of cyclin E. Nat Genet 23: 245248.

del Pozo, J.C., Dharmasiri, S., Hellmann, H., Walker, L., Gray, W.M., and Estelle, M. (2002) AXR1-ECR1dependent conjugation of RUB1 to the Arabidopsis Cullin AtCUL1 is required for auxin response. Plant Cell 14: 421-433.

Deng, X.W., Dubiel, W., Wei, N., Hofmann, K., and Mundt, K. (2000) Unified nomenclature for the COP9 signalosome and its subunits: an essential regulator of development. Trends Genet 16: 289.

Deshaies, R.J. (1999) SCF and Cullin/Ring H2-based ubiquitin ligases. Annu Rev Cell Dev Biol 15: 435-467.

Dong, J., Qiu, H., Garcia-Barrio, M., Anderson, J., and Hinnebusch, A.G. (2000) Uncharged tRNA activates GCN2 by displacing the protein kinase moiety from a bipartite tRNA-binding domain. Mol Cell 6: 269279.

Doronkin, S., Djagaeva, I., and Beckendorf, S.K. (2002) CSN5/Jab1 mutations affect axis formation in the Drosophila oocyte by activating a meiotic checkpoint. Development 129: 5053-5064.

Doronkin, S., Djagaeva, I., and Beckendorf, S.K. (2003) The COP9 signalosome promotes degradation of Cyclin E during early Drosophila oogenesis. Dev Cell 4: 699-710. 
Drysdale, C.M., Duenas, E., Jackson, B.M., Reusser, U., Braus, G.H., and Hinnebusch, A.G. (1995) The transcriptional activator GCN4 contains multiple activation domains that are critically dependent on hydrophobic amino acids. Mol Cell Biol 15: 1220-1233.

Drysdale, C.M., Jackson, B.M., McVeigh, R., Klebanow, E.R., Bai, Y., Kokubo, T., Swanson, M., Nakatani, Y., Weil, P.A., and Hinnebusch, A.G. (1998) The Gcn4p activation domain interacts specifically in vitro with RNA polymerase II holoenzyme, TFIID, and the Adap-Gen5p coactivator complex. Mol Cell Biol 18: 1711-1724.

Dunn, C., Wiltshire, C., MacLaren, A., and Gillespie, D.A. (2002) Molecular mechanism and biological functions of c-Jun N-terminal kinase signalling via the c-Jun transcription factor. Cell Signal 14: 585-593.

Ebbole, D.J., Paluh, J.L., Plamann, M., Sachs, M.S., and Yanofsky, C. (1991) cpc-1, the general regulatory gene for genes of amino acid biosynthesis in Neurospora crassa, is differentially expressed during the asexual life cycle. Mol Cell Biol 11: 928-934.

Eckert, S.E., Hoffmann, B., Wanke, C., and Braus, G.H. (1999) Sexual development of Aspergillus nidulans in tryptophan auxotrophic strains. Arch Microbiol 172: 157-166.

Eckert, S.E., Kübler, E., Hoffmann, B., and Braus, G.H. (2000) The tryptophan synthase-encoding $\operatorname{trpB}$ gene of Aspergillus nidulans is regulated by the cross-pathway control system. Mol Gen Genet 263: 867-876.

Elsea, S.H., Mykytyn, K., Ferrell, K., Coulter, K.L., Das, P., Dubiel, W., Patel, P.I., and Metherall, J.E. (1999) Hemizygosity for the COP9 signalosome subunit gene, SGN3, in the Smith-Magenis syndrome. Am J Med Genet 87: 342-348.

Engelberg, D., Klein, C., Martinetto, H., Struhl, K., and Karin, M. (1994) The UV response involving the Ras signaling pathway and AP-1 transcription factors is conserved between yeast and mammals. Cell 77: 381390.

Feng, S., Ma, L., Wang, X., Xie, D., Dinesh-Kumar, S.P., Wei, N., and Deng, X.W. (2003) The COP9 signalosome interacts physically with SCF COI1 and modulates jasmonate responses. Plant Cell 15: 1083-1094.

Filetici, P., Aranda, C., Gonzalez, A., and Ballario, P. (1998) GCN5, a yeast transcriptional coactivator, induces chromatin reconfiguration of HIS3 promoter in vivo. Biochem Biophys Res Commun 242: 84-87.

Freilich, S., Oron, E., Kapp, Y., Nevo-Caspi, Y., Orgad, S., Segal, D., and Chamovitz, D.A. (1999) The COP9 signalosome is essential for development of Drosophila melanogaster. Curr Biol 9: 1187-1190.

Fu, H., Reis, N., Lee, Y., Glickman, M.H., and Vierstra, R.D. (2001) Subunit interaction maps for the regulatory particle of the 26S proteasome and the COP9 signalosome. EMBO J 20: 7096-7107.

Garcia-Barrio, M., Dong, J., Ufano, S., and Hinnebusch, A.G. (2000) Association of GCN1-GCN20 regulatory complex with the N-terminus of eIF2alpha kinase GCN2 is required for GCN2 activation. EMBO J 19: 1887-1899.

Glickman, M.H., Rubin, D.M., Coux, O., Wefes, I., Pfeifer, G., Cjeka, Z., Baumeister, W., Fried, V.A., and Finley, D. (1998) A subcomplex of the proteasome regulatory particle required for ubiquitin-conjugate degradation and related to the COP9-signalosome and eIF3. Cell 94: 615-623.

Goossens, A., Dever, T.E., Pascual-Ahuir, A., and Serrano, R. (2001) The protein kinase Gcn2p mediates sodium toxicity in yeast. J Biol Chem 276: 30753-30760. 
Green, M.R. (2000) TBP-associated factors (TAFIIs): multiple, selective transcriptional mediators in common complexes. Trends Biochem Sci 25: 59-63.

Greenberg, F., Lewis, R.A., Potocki, L., Glaze, D., Parke, J., Killian, J., Murphy, M.A., Williamson, D., Brown, F., Dutton, R., McCluggage, C., Friedman, E., Sulek, M., and Lupski, J.R. (1996) Multi-disciplinary clinical study of Smith-Magenis syndrome (deletion 17p11.2). Am J Med Genet 62: 247-254.

Groisman, R., Polanowska, J., Kuraoka, I., Sawada, J., Saijo, M., Drapkin, R., Kisselev, A.F., Tanaka, K., and Nakatani, Y. (2003) The ubiquitin ligase activity in the DDB2 and CSA complexes is differentially regulated by the COP9 signalosome in response to DNA damage. Cell 113: 357-367.

Grundmann, O., Mösch, H.U., and Braus, G.H. (2001) Repression of GCN4 mRNA translation by nitrogen starvation in Saccharomyces cerevisiae. J Biol Chem 276: 25661-25671.

Gusmaroli, G., Feng, S., and Deng, X.W. (2004) The Arabidopsis CSN5A and CSN5B subunits are present in distinct COP9 signalosome complexes, and mutations in their JAMM domains exhibit differential dominant negative effects on development. Plant Cell 16: 2984-3001.

Harari-Steinberg, O., and Chamovitz, D.A. (2004) The COP9 signalosome: mediating between kinase signaling and protein degradation. Curr Protein Pept Sci 5: 185-189.

Harashima, S., and Hinnebusch, A.G. (1986) Multiple GCD genes required for repression of GCN4, a transcriptional activator of amino acid biosynthetic genes in Saccharomyces cerevisiae. Mol Cell Biol 6: 3990-3998.

He, Q., Cheng, P., and Liu, Y. (2005) The COP9 signalosome regulates the Neurospora circadian clock by controlling the stability of the SCFFWD-1 complex. Genes Dev. 19:1518-1531

Hershko, A., and Ciechanover, A. (1998) The ubiquitin system. Annu Rev Biochem 67: 425-479.

Hinnebusch, A.G. (1986) The general control of amino acid biosynthetic genes in the yeast Saccharomyces cerevisiae. CRC Crit Rev Biochem 21: 277-317.

Hinnebusch, A.G. (1997) Translational regulation of yeast GCN4. A window on factors that control initiatortRNA binding to the ribosome. J Biol Chem 272: 21661-21664.

Hinnebusch, A.G., and Natarajan, K. (2002) Gcn4p, a master regulator of gene expression, is controlled at multiple levels by diverse signals of starvation and stress. Eukaryot Cell 1: 22-32.

Hoareau Alves, K., Bochard, V., Rety, S., and Jalinot, P. (2002) Association of the mammalian protooncoprotein Int-6 with the three protein complexes eIF3, COP9 signalosome and 26S proteasome. FEBS Lett 527: 15-21.

Hochstrasser, M. (2000) Evolution and function of ubiquitin-like protein-conjugation systems. Nat Cell Biol 2 : E153-157.

Hoffmann, B., Mösch, H.U., Sattlegger, E., Barthelmess, I.B., Hinnebusch, A., and Braus, G.H. (1999) The WD protein $\mathrm{Cpc} 2 \mathrm{p}$ is required for repression of Gcn4 protein activity in yeast in the absence of amino-acid starvation. Mol Microbiol 31: 807-822.

Hoffmann, B., Wanke, C., Lapaglia, S.K., and Braus, G.H. (2000) c-Jun and RACK1 homologues regulate a control point for sexual development in Aspergillus nidulans. Mol Microbiol 37: 28-41. 


\section{Introduction}

Hoffmann, B., Valerius, O., Andermann, M., and Braus, G.H. (2001) Transcriptional autoregulation and inhibition of mRNA translation of amino acid regulator gene cpcA of filamentous fungus Aspergillus nidulans. Mol Biol Cell 12: 2846-2857.

Hofmann, K., and Bucher, P. (1998) The PCI domain: a common theme in three multiprotein complexes. Trends Biochem Sci 23: 204-205.

Holstege, F.C., Jennings, E.G., Wyrick, J.J., Lee, T.I., Hengartner, C.J., Green, M.R., Golub, T.R., Lander, E.S., and Young, R.A. (1998) Dissecting the regulatory circuitry of a eukaryotic genome. Cell 95: 717-728.

Hope, I.A., and Struhl, K. (1985) GCN4 protein, synthesized in vitro, binds HIS3 regulatory sequences: implications for general control of amino acid biosynthetic genes in yeast. Cell 43: 177-188.

Hope, I.A., and Struhl, K. (1986) Functional dissection of a eukaryotic transcriptional activator protein, GCN4 of yeast. Cell 46: 885-894.

Irniger, S., and Braus, G.H. (2003) Controlling transcription by destruction: the regulation of yeast Gcn4p stability. Curr Genet 44: 8-18.

Isono, E., Saeki, Y., Yokosawa, H., and Toh-e, A. (2004) Rpn7 Is required for the structural integrity of the $26 \mathrm{~S}$ proteasome of Saccharomyces cerevisiae. J Biol Chem 279: 27168-27176.

Ito, T. (2003) Nucleosome assembly and remodeling. Curr Top Microbiol Immunol 274: 1-22.

Jia, M.H., Larossa, R.A., Lee, J.M., Rafalski, A., Derose, E., Gonye, G., and Xue, Z. (2000) Global expression profiling of yeast treated with an inhibitor of amino acid biosynthesis, sulfometuron methyl. Physiol Genomics 3: 83-92.

John, M., Leppik, R., Busch, S.J., Granger-Schnarr, M., and Schnarr, M. (1996) DNA binding of Jun and Fos bZip domains: homodimers and heterodimers induce a DNA conformational change in solution. Nucleic Acids Res 24: 4487-4494.

Kabe, Y., Goto, M., Shima, D., Imai, T., Wada, T., Morohashi, K., Shirakawa, M., Hirose, S., and Handa, H. (1999) The role of human MBF1 as a transcriptional coactivator. J Biol Chem 274: 34196-34202.

Käfer, E. (1977) The anthranilate synthetase enzyme complex and the trifunctional trpC gene of Aspergillus. Can J Genet Cytol 19: 723-738.

Kapelari, B., Bech-Otschir, D., Hegerl, R., Schade, R., Dumdey, R., and Dubiel, W. (2000) Electron microscopy and subunit-subunit interaction studies reveal a first architecture of COP9 signalosome. J Mol Biol 300: $1169-1178$

Karniol, B., Yahalom, A., Kwok, S., Tsuge, T., Matsui, M., Deng, X.W., and Chamovitz, D.A. (1998) The Arabidopsis homologue of an eIF3 complex subunit associates with the COP9 complex. FEBS Lett 439: 173-179.

Karniol, B., Malec, P., and Chamovitz, D.A. (1999) Arabidopsis FUSCA5 encodes a novel phosphoprotein that is a component of the COP9 complex. Plant Cell 11: 839-848.

Khan, A.U., and Krishnamurthy, S. (2005) Histone modifications as key regulators of transcription. Front Biosci 10: $866-872$. 
Khochbin, S., and Kao, H.Y. (2001) Histone deacetylase complexes: functional entities or molecular reservoirs. FEBS Lett 494: 141-144.

Kim, T., Hofmann, K., von Arnim, A.G., and Chamovitz, D.A. (2001) PCI complexes: pretty complex interactions in diverse signaling pathways. Trends Plant Sci 6: 379-386.

Kim, Y.J., Bjorklund, S., Li, Y., Sayre, M.H., and Kornberg, R.D. (1994) A multiprotein mediator of transcriptional activation and its interaction with the C-terminal repeat domain of RNA polymerase II. Cell 77: 599-608.

Kleemann, R., Hausser, A., Geiger, G., Mischke, R., Burger-Kentischer, A., Flieger, O., Johannes, F.J., Roger, T., Calandra, T., Kapurniotu, A., Grell, M., Finkelmeier, D., Brunner, H., and Bernhagen, J. (2000) Intracellular action of the cytokine MIF to modulate AP-1 activity and the cell cycle through Jab1. Nature 408: 211-216.

Kleinschmidt, M., Grundmann, O., Bluthgen, N., Mösch, H.U., and Braus, G.H. (2005) Transcriptional profiling of Saccharomyces cerevisiae cells under adhesion-inducing conditions. Mol Genet Genomics 273: 382393.

Kominami, K., Ochotorena, I., and Toda, T. (1998) Two F-box/WD-repeat proteins Pop1 and Pop2 form heteroand homo-complexes together with cullin-1 in the fission yeast SCF (Skp1-Cullin-1-F-box) ubiquitin ligase. Genes Cells 3: 721-735.

Kornberg, R.D., and Lorch, Y. (1991) Irresistible force meets immovable object: transcription and the nucleosome. Cell 67: 833-836.

Kornitzer, D., Raboy, B., Kulka, R.G., and Fink, G.R. (1994) Regulated degradation of the transcription factor Gcn4. EMBO J 13: 6021-6030.

Kouzarides, T., and Ziff, E. (1989) Leucine zippers of fos, jun and GCN4 dictate dimerization specificity and thereby control DNA binding. Nature 340: 568-571.

Kubota, H., Sakaki, Y., and Ito, T. (2000) GI domain-mediated association of the eukaryotic initiation factor 2alpha kinase GCN2 with its activator GCN1 is required for general amino acid control in budding yeast. J Biol Chem 275: 20243-20246.

Kubota, H., Ota, K., Sakaki, Y., and Ito, T. (2001) Budding yeast GCN1 binds the GI domain to activate the eIF2alpha kinase GCN2. J Biol Chem 276: 17591-17596.

Kwok, S.F., Staub, J.M., and Deng, X.W. (1999) Characterization of two subunits of Arabidopsis 19S proteasome regulatory complex and its possible interaction with the COP9 complex. J Mol Biol 285: 8595.

Laplaza, J.M., Bostick, M., Scholes, D.T., Curcio, M.J., and Callis, J. (2004) Saccharomyces cerevisiae ubiquitin-like protein Rub1 conjugates to cullin proteins Rtt101 and Cul3 in vivo. Biochem J 377: 459467.

Lier, S., and Paululat, A. (2002) The proteasome regulatory particle subunit Rpn6 is required for Drosophila development and interacts physically with signalosome subunit Alien/CSN2. Gene 298: 109-119.

Liu, C., Powell, K.A., Mundt, K., Wu, L., Carr, A.M., and Caspari, T. (2003) Cop9/signalosome subunits and Pcu4 regulate ribonucleotide reductase by both checkpoint-dependent and -independent mechanisms. Genes Dev 17: 1130-1140. 
Luger, K., Mader, A.W., Richmond, R.K., Sargent, D.F., and Richmond, T.J. (1997) Crystal structure of the nucleosome core particle at 2.8 A resolution. Nature 389: 251-260.

Lusk, C.P., Makhnevych, T., and Wozniak, R.W. (2004) New ways to skin a kap: mechanisms for controlling nuclear transport. Biochem Cell Biol 82: 618-625.

Lyapina, S., Cope, G., Shevchenko, A., Serino, G., Tsuge, T., Zhou, C., Wolf, D.A., Wei, N., and Deshaies, R.J. (2001) Promotion of NEDD-CUL1 conjugate cleavage by COP9 signalosome. Science 292: 1382-1385.

Lykke-Andersen, K., Schaefer, L., Menon, S., Deng, X.W., Miller, J.B., and Wei, N. (2003) Disruption of the COP9 signalosome Csn2 subunit in mice causes deficient cell proliferation, accumulation of p53 and cyclin E, and early embryonic death. Mol Cell Biol 23: 6790-6797.

Ma, L., Zhao, H., and Deng, X.W. (2003) Analysis of the mutational effects of the COP/DET/FUS loci on genome expression profiles reveals their overlapping yet not identical roles in regulating Arabidopsis seedling development. Development 130: 969-981.

Matangkasombut, O., Auty, R., and Buratowski, S. (2004) Structure and function of the TFIID complex. $A d v$ Protein Chem 67: 67-92.

Maytal-Kivity, V., Piran, R., Pick, E., Hofmann, K., and Glickman, M.H. (2002a) COP9 signalosome components play a role in the mating pheromone response of S. cerevisiae. EMBO Rep 3: 1215-1221.

Maytal-Kivity, V., Reis, N., Hofmann, K., and Glickman, M.H. (2002b) MPN+, a putative catalytic motif found in a subset of MPN domain proteins from eukaryotes and prokaryotes, is critical for Rpn11 function. BMC Biochem 3: 28.

Meimoun, A., Holtzman, T., Weissman, Z., McBride, H.J., Stillman, D.J., Fink, G.R., and Kornitzer, D. (2000) Degradation of the transcription factor Gcn4 requires the kinase Pho85 and the SCF(CDC4) ubiquitinligase complex. Mol Biol Cell 11: 915-927.

Michel, J.J., McCarville, J.F., and Xiong, Y. (2003) A role for Saccharomyces cerevisiae Cul8 ubiquitin ligase in proper anaphase progression. J Biol Chem 278: 22828-22837.

Misera, S., Muller, A.J., Weiland-Heidecker, U., and Jurgens, G. (1994) The FUSCA genes of Arabidopsis: negative regulators of light responses. Mol Gen Genet 244: 242-252.

Mundt, K.E., Porte, J., Murray, J.M., Brikos, C., Christensen, P.U., Caspari, T., Hagan, I.M., Millar, J.B., Simanis, V., Hofmann, K., and Carr, A.M. (1999) The COP9/signalosome complex is conserved in fission yeast and has a role in S phase. Curr Biol 9: 1427-1430.

Mundt, K.E., Liu, C., and Carr, A.M. (2002) Deletion mutants in COP9/signalosome subunits in fission yeast Schizosaccharomyces pombe display distinct phenotypes. Mol Biol Cell 13: 493-502.

Natarajan, K., Jackson, B.M., Zhou, H., Winston, F., and Hinnebusch, A.G. (1999) Transcriptional activation by Gcn4p involves independent interactions with the SWI/SNF complex and the SRB/mediator. Mol Cell 4: 657-664.

Natarajan, K., Meyer, M.R., Jackson, B.M., Slade, D., Roberts, C., Hinnebusch, A.G., and Marton, M.J. (2001) Transcriptional profiling shows that Gen4p is a master regulator of gene expression during amino acid starvation in yeast. Mol Cell Biol 21: 4347-4368. 
Neely, K.E., Hassan, A.H., Brown, C.E., Howe, L., and Workman, J.L. (2002) Transcription activator interactions with multiple SWI/SNF subunits. Mol Cell Biol 22: 1615-1625.

Noll, M., and Kornberg, R.D. (1977) Action of micrococcal nuclease on chromatin and the location of histone H1. J Mol Biol 109: 393-404.

Ohta, T., Michel, J.J., Schottelius, A.J., and Xiong, Y. (1999) ROC1, a homolog of APC11, represents a family of cullin partners with an associated ubiquitin ligase activity. Mol Cell 3: 535-541.

Ohta, T., and Xiong, Y. (2001) Phosphorylation- and Skp1-independent in vitro ubiquitination of E2F1 by multiple ROC-cullin ligases. Cancer Res 61: 1347-1353.

Oliphant, A.R., Brandl, C.J., and Struhl, K. (1989) Defining the sequence specificity of DNA-binding proteins by selecting binding sites from random-sequence oligonucleotides: analysis of yeast GCN4 protein. $\mathrm{Mol}$ Cell Biol 9: 2944-2949.

Oron, E., Mannervik, M., Rencus, S., Harari-Steinberg, O., Neuman-Silberberg, S., Segal, D., and Chamovitz, D.A. (2002) COP9 signalosome subunits 4 and 5 regulate multiple pleiotropic pathways in Drosophila melanogaster. Development 129: 4399-4409.

Osaka, F., Saeki, M., Katayama, S., Aida, N., Toh, E.A., Kominami, K., Toda, T., Suzuki, T., Chiba, T., Tanaka, K., and Kato, S. (2000) Covalent modifier NEDD8 is essential for SCF ubiquitin-ligase in fission yeast. EMBO J 19: 3475-3484.

Pan, Z.Q., Kentsis, A., Dias, D.C., Yamoah, K., and Wu, K. (2004) Nedd8 on cullin: building an expressway to protein destruction. Oncogene 23: 1985-1997.

Peng, Z., Serino, G., and Deng, X.W. (2001a) Molecular characterization of subunit 6 of the COP9 signalosome and its role in multifaceted developmental processes in Arabidopsis. Plant Cell 13: 2393-2407.

Peng, Z., Serino, G., and Deng, X.W. (2001b) A role of Arabidopsis COP9 signalosome in multifaceted developmental processes revealed by the characterization of its subunit 3. Development 128: 4277-4288.

Peng, Z., Staub, J.M., Serino, G., Kwok, S.F., Kurepa, J., Bruce, B.D., Vierstra, R.D., Wei, N., and Deng, X.W. (2001c) The cellular level of PR500, a protein complex related to the 19S regulatory particle of the proteasome, is regulated in response to stresses in plants. Mol Biol Cell 12: 383-392.

Peng, Z., Shen, Y., Feng, S., Wang, X., Chitteti, B.N., Vierstra, R.D., and Deng, X.W. (2003) Evidence for a physical association of the COP9 signalosome, the proteasome, and specific SCF E3 ligases in vivo. Curr Biol 13: R504-505.

Piotrowska, M., Kruszewska, A., and Paszewski, A. (1980) Effect of regulatory mutations of sulphur metabolism on the levels of cysteine- and homocysteine-synthesizing enzymes in Neurospora crassa. Acta Biochim Pol 27: 395-403.

Potocki, L., Chen, K.S., and Lupski, J.R. (1999) Subunit 3 of the COP9 signal transduction complex is conserved from plants to humans and maps within the smith-magenis syndrome critical region in 17p11.2. Genomics 57: 180-182.

Potocki, L., Glaze, D., Tan, D.X., Park, S.S., Kashork, C.D., Shaffer, L.G., Reiter, R.J., and Lupski, J.R. (2000) Circadian rhythm abnormalities of melatonin in Smith-Magenis syndrome. J Med Genet 37: 428-433. 
Pries, R., Bömeke, K., Irniger, S., Grundmann, O., and Braus, G.H. (2002) Amino acid-dependent Gcn4p stability regulation occurs exclusively in the yeast nucleus. Eukaryot Cell 1: 663-672.

Pries, R., Bömeke, K., Draht, O., Künzler, M., and Braus, G.H. (2004) Nuclear import of yeast Gcn4p requires karyopherins Srp1p and Kap95p. Mol Genet Genomics 271: 257-266.

Qiu, H., Dong, J., Hu, C., Francklyn, C.S., and Hinnebusch, A.G. (2001) The tRNA-binding moiety in GCN2 contains a dimerization domain that interacts with the kinase domain and is required for tRNA binding and kinase activation. EMBO J 20: 1425-1438.

Qiu, H., Hu, C., Yoon, S., Natarajan, K., Swanson, M.J., and Hinnebusch, A.G. (2004) An array of coactivators is required for optimal recruitment of TATA binding protein and RNA polymerase II by promoter-bound Gcn4p. Mol Cell Biol 24: 4104-4117.

Quinn, J., Fyrberg, A.M., Ganster, R.W., Schmidt, M.C., and Peterson, C.L. (1996) DNA-binding properties of the yeast SWI/SNF complex. Nature 379: 844-847.

Rechsteiner, M., and Rogers, S.W. (1996) PEST sequences and regulation by proteolysis. Trends Biochem Sci 21: $267-271$.

Roberts, S.M., and Winston, F. (1997) Essential functional interactions of SAGA, a Saccharomyces cerevisiae complex of Spt, Ada, and Gen5 proteins, with the Snf/Swi and Srb/mediator complexes. Genetics 147: 451-465.

Rolfes, R.J., and Hinnebusch, A.G. (1993) Translation of the yeast transcriptional activator GCN4 is stimulated by purine limitation: implications for activation of the protein kinase GCN2. Mol Cell Biol 13: 50995111.

Rotenberg, S.A., and Sun, X.G. (1998) Photoinduced inactivation of protein kinase C by dequalinium identifies the RACK-1-binding domain as a recognition site. J Biol Chem 273: 2390-2395.

Sachs, M.S. (1996) General and cross-pathway control of amino acid biosynthesis. In The Mykota. Esser, K. and Lemke, P.A. (eds). Berlin, Heidelberg, New York: Springer-Verlag, pp. 315-345.

Sattlegger, E., and Hinnebusch, A.G. (2000) Separate domains in GCN1 for binding protein kinase GCN2 and ribosomes are required for GCN2 activation in amino acid-starved cells. EMBO J 19: 6622-6633.

Scheel, H., and Hofmann, K. (2005) Prediction of a common structural scaffold for proteasome lid, COP9signalosome and eIF3 complexes. BMC Bioinformatics 6: 71.

Schwechheimer, C., and Deng, X.W. (2001) COP9 signalosome revisited: a novel mediator of protein degradation. Trends Cell Biol 11: 420-426.

Schwechheimer, C., Serino, G., Callis, J., Crosby, W.L., Lyapina, S., Deshaies, R.J., Gray, W.M., Estelle, M., and Deng, X.W. (2001) Interactions of the COP9 signalosome with the E3 ubiquitin ligase SCFTIRI in mediating auxin response. Science 292: 1379-1382.

Schwechheimer, C., Serino, G., and Deng, X.W. (2002) Multiple ubiquitin ligase-mediated processes require COP9 signalosome and AXR1 function. Plant Cell 14: 2553-2563.

Seeger, M., Kraft, R., Ferrell, K., Bech-Otschir, D., Dumdey, R., Schade, R., Gordon, C., Naumann, M., and Dubiel, W. (1998) A novel protein complex involved in signal transduction possessing similarities to $26 \mathrm{~S}$ proteasome subunits. Faseb J 12: 469-478. 
Seeger, M., Gordon, C., and Dubiel, W. (2001) Protein stability: the COP9 signalosome gets in on the act. Curr Biol 11: R643-646.

Serino, G., Tsuge, T., Kwok, S., Matsui, M., Wei, N., and Deng, X.W. (1999) Arabidopsis cop8 and fus4 mutations define the same gene that encodes subunit 4 of the COP9 signalosome. Plant Cell 11: 19671980.

Serino, G., and Deng, X.W. (2003) THE COP9 SIGNALOSOME: Regulating Plant Development Through the Control of Proteolysis. Annu Rev Plant Physiol Plant Mol Biol 54: 165-182.

Serino, G., Su, H., Peng, Z., Tsuge, T., Wei, N., Gu, H., and Deng, X.W. (2003) Characterization of the last subunit of the Arabidopsis COP9 signalosome: implications for the overall structure and origin of the complex. Plant Cell 15: 719-731.

Serlupi-Crescenzi, O., Kurtz, M.B., and Champe, S.P. (1983) Developmental defects resulting from arginine auxotrophy in Aspergillus nidulans. J Gen Microbiol 129: 3535-3544.

Shalev, A., Valasek, L., Pise-Masison, C.A., Radonovich, M., Phan, L., Clayton, J., He, H., Brady, J.N., Hinnebusch, A.G., and Asano, K. (2001) Saccharomyces cerevisiae protein Pci8p and human protein eIF3e/Int-6 interact with the eIF3 core complex by binding to cognate eIF3b subunits. J Biol Chem 276: 34948-34957.

Sharpless, N.E., and DePinho, R.A. (2002) p53: good cop/bad cop. Cell 110: 9-12.

Shaw, B.R., Herman, T.M., Kovacic, R.T., Beaudreau, G.S., and Van Holde, K.E. (1976) Analysis of subunit organization in chicken erythrocyte chromatin. Proc Natl Acad Sci U S A 73: 505-509.

Shemer, R., Meimoun, A., Holtzman, T., and Kornitzer, D. (2002) Regulation of the transcription factor Gen4 by Pho85 cyclin PCL5. Mol Cell Biol 22: 5395-5404.

Sherr, C.J., and Roberts, J.M. (1999) CDK inhibitors: positive and negative regulators of G1 -phase progression. Genes Dev 13: 1501-1512.

Slingerland, J., and Pagano, M. (2000) Regulation of the cdk inhibitor p27 and its deregulation in cancer. J Cell Physiol 183: 10-17.

Smith, P., Leung-Chiu, W.M., Montgomery, R., Orsborn, A., Kuznicki, K., Gressman-Coberly, E., Mutapcic, L., and Bennett, K. (2002) The GLH proteins, Caenorhabditis elegans P granule components, associate with CSN-5 and KGB-1, proteins necessary for fertility, and with ZYX-1, a predicted cytoskeletal protein. Dev Biol 251: 333-347.

Suh, G.S., Poeck, B., Chouard, T., Oron, E., Segal, D., Chamovitz, D.A., and Zipursky, S.L. (2002) Drosophila JAB1/CSN5 acts in photoreceptor cells to induce glial cells. Neuron 33: 35-46.

Sun, Y., Wilson, M.P., and Majerus, P.W. (2002) Inositol 1,3,4-trisphosphate 5/6-kinase associates with the COP9 signalosome by binding to CSN1. J Biol Chem 277: 45759-45764.

Takemaru, K., Harashima, S., Ueda, H., and Hirose, S. (1998) Yeast coactivator MBF1 mediates GCN4dependent transcriptional activation. Mol Cell Biol 18: 4971-4976.

Tang, Z., Li, B., Bharadwaj, R., Zhu, H., Ozkan, E., Hakala, K., Deisenhofer, J., and Yu, H. (2001) APC2 Cullin protein and APC11 RING protein comprise the minimal ubiquitin ligase module of the anaphasepromoting complex. Mol Biol Cell 12: 3839-3851. 
Tateishi, K., Omata, M., Tanaka, K., and Chiba, T. (2001) The NEDD8 system is essential for cell cycle progression and morphogenetic pathway in mice. J Cell Biol 155: 571-579.

Timmers, H.T., and Tora, L. (2005) SAGA unveiled. Trends Biochem Sci 30: 7-10.

Tomoda, K., Kubota, Y., Arata, Y., Mori, S., Maeda, M., Tanaka, T., Yoshida, M., Yoneda-Kato, N., and Kato, J.Y. (2002) The cytoplasmic shuttling and subsequent degradation of p27Kip1 mediated by Jab1/CSN5 and the COP9 signalosome complex. J Biol Chem 277: 2302-2310.

Tomoda, K., Yoneda-Kato, N., Fukumoto, A., Yamanaka, S., and Kato, J.Y. (2004) Multiple functions of Jab1 are required for early embryonic development and growth potential in mice. J Biol Chem 279: 4301343018.

Tsuge, T., Matsui, M., and Wei, N. (2001) The subunit 1 of the COP9 signalosome suppresses gene expression through its N-terminal domain and incorporates into the complex through the PCI domain. J Mol Biol 305: $1-9$.

Turner, R., and Tjian, R. (1989) Leucine repeats and an adjacent DNA binding domain mediate the formation of functional cFos-cJun heterodimers. Science 243: 1689-1694.

Tyers, M., and Jorgensen, P. (2000) Proteolysis and the cell cycle: with this RING I do thee destroy. Curr Opin Genet Dev 10: 54-64.

Uhle, S., Medalia, O., Waldron, R., Dumdey, R., Henklein, P., Bech-Otschir, D., Huang, X., Berse, M., Sperling, J., Schade, R., and Dubiel, W. (2003) Protein kinase CK2 and protein kinase D are associated with the COP9 signalosome. EMBO J 22: 1302-1312.

Valasek, L., Phan, L., Schoenfeld, L.W., Valaskova, V., and Hinnebusch, A.G. (2001) Related eIF3 subunits TIF32 and HCR1 interact with an RNA recognition motif in PRT1 required for eIF3 integrity and ribosome binding. EMBO J 20: 891-904.

Valenzuela, L., Aranda, C., and Gonzalez, A. (2001) TOR modulates GCN4-dependent expression of genes turned on by nitrogen limitation. J Bacteriol 183: 2331-2334.

Valerius, O., Draht, O., Kübler, E., Adler, K., Hoffmann, B., and Braus, G.H. (2001) Regulation of hisHF transcription of Aspergillus nidulans by adenine and amino acid limitation. Fungal Genet Biol 32: 21-31.

Verger, A., and Crossley, M. (2004) Chromatin modifiers in transcription and DNA repair. Cell Mol Life Sci 61: 2154-2162.

Verma, R., Aravind, L., Oania, R., McDonald, W.H., Yates, J.R., 3rd, Koonin, E.V., and Deshaies, R.J. (2002) Role of Rpn11 metalloprotease in deubiquitination and degradation by the $26 \mathrm{~S}$ proteasome. Science 298 : 611-615.

Wang, X., Kang, D., Feng, S., Serino, G., Schwechheimer, C., and Wei, N. (2002) CSN1 N-terminal-dependent activity is required for Arabidopsis development but not for Rub1/Nedd8 deconjugation of cullins: a structure-function study of CSN1 subunit of COP9 signalosome. Mol Biol Cell 13: 646-655.

Wang, X., Feng, S., Nakayama, N., Crosby, W.L., Irish, V., Deng, X.W., and Wei, N. (2003) The COP9 signalosome interacts with SCF UFO and participates in Arabidopsis flower development. Plant Cell 15: 1071-1082. 
Wang, Y., Penfold, S., Tang, X., Hattori, N., Riley, P., Harper, J.W., Cross, J.C., and Tyers, M. (1999) Deletion of the Cul1 gene in mice causes arrest in early embryogenesis and accumulation of cyclin E. Curr Biol 9: 1191-1194.

Wee, S., Hetfeld, B., Dubiel, W., and Wolf, D.A. (2002) Conservation of the COP9/signalosome in budding yeast. BMC Genet 3: 15 .

Wee, S., Geyer, R.K., Toda, T., and Wolf, D.A. (2005) CSN facilitates Cullin-RING ubiquitin ligase function by counteracting autocatalytic adapter instability. Nat Cell Biol 7: 387-391.

Wei, N., and Deng, X.W. (1992) COP9: a new genetic locus involved in light-regulated development and gene expression in Arabidopsis. Plant Cell 4: 1507-1518.

Wei, N., Chamovitz, D.A., and Deng, X.W. (1994) Arabidopsis COP9 is a component of a novel signaling complex mediating light control of development. Cell 78: 117-124.

Wei, N., Tsuge, T., Serino, G., Dohmae, N., Takio, K., Matsui, M., and Deng, X.W. (1998) The COP9 complex is conserved between plants and mammals and is related to the $26 \mathrm{~S}$ proteasome regulatory complex. Curr Biol 8: 919-922.

Wei, N., and Deng, X.W. (2003) The COP9 signalosome. Annu Rev Cell Dev Biol 19: 261-286.

Wek, S.A., Zhu, S., and Wek, R.C. (1995) The histidyl-tRNA synthetase-related sequence in the eIF-2 alpha protein kinase GCN2 interacts with tRNA and is required for activation in response to starvation for different amino acids. Mol Cell Biol 15: 4497-4506.

Wilson, M.P., Sun, Y., Cao, L., and Majerus, P.W. (2001) Inositol 1,3,4-trisphosphate 5/6-kinase is a protein kinase that phosphorylates the transcription factors c-Jun and ATF-2. J Biol Chem 276: 40998-41004.

Wolf, D.A., Zhou, C., and Wee, S. (2003) The COP9 signalosome: an assembly and maintenance platform for cullin ubiquitin ligases? Nat Cell Biol 5: 1029-1033.

Yahalom, A., Kim, T.H., Winter, E., Karniol, B., von Arnim, A.G., and Chamovitz, D.A. (2001) Arabidopsis eIF3e (INT-6) associates with both eIF3c and the COP9 signalosome subunit CSN7. J Biol Chem 276: 334-340.

Yan, J., Walz, K., Nakamura, H., Carattini-Rivera, S., Zhao, Q., Vogel, H., Wei, N., Justice, M.J., Bradley, A., and Lupski, J.R. (2003) COP9 signalosome subunit 3 is essential for maintenance of cell proliferation in the mouse embryonic epiblast. Mol Cell Biol 23: 6798-6808.

Yang, X., Menon, S., Lykke-Andersen, K., Tsuge, T., Di, X., Wang, X., Rodriguez-Suarez, R.J., Zhang, H., and Wei, N. (2002) The COP9 signalosome inhibits p27(kip1) degradation and impedes G1-S phase progression via deneddylation of SCF Cul1. Curr Biol 12: 667-672.

Yao, T., and Cohen, R.E. (2002) A cryptic protease couples deubiquitination and degradation by the proteasome. Nature 419: 403-407.

Yelton, M.M., Hamer, J.E., de Souza, E.R., Mullaney, E.J., and Timberlake, W.E. (1983) Developmental regulation of the Aspergillus nidulans trpC gene. Proc Natl Acad Sci U S A 80: 7576-7580.

Zheng, J., Yang, X., Harrell, J.M., Ryzhikov, S., Shim, E.H., Lykke-Andersen, K., Wei, N., Sun, H., Kobayashi, R., and Zhang, H. (2002) CAND1 binds to unneddylated CUL1 and regulates the formation of SCF ubiquitin E3 ligase complex. Mol Cell 10: 1519-1526. 


\section{Introduction}

Zhou, C., Seibert, V., Geyer, R., Rhee, E., Lyapina, S., Cope, G., Deshaies, R.J., and Wolf, D.A. (2001) The fission yeast COP9/signalosome is involved in cullin modification by ubiquitin-related Ned8p. BMC Biochem 2: 7.

Zhou, C., Wee, S., Rhee, E., Naumann, M., Dubiel, W., and Wolf, D.A. (2003) Fission yeast COP9/signalosome suppresses cullin activity through recruitment of the deubiquitylating enzyme Ubp12p. Mol Cell 11: $927-$ 938.

Zhu, S., Sobolev, A.Y., and Wek, R.C. (1996) Histidyl-tRNA synthetase-related sequences in GCN2 protein kinase regulate in vitro phosphorylation of eIF-2. J Biol Chem 271: 24989-24994. 


\section{Chapter 2}

Aspergillus nidulans sexual development is dependent on COP9 deneddylation activity

\subsection{Abstract}

The COP9 signalosome (CSN) is a multifunctional protein complex essential for the development of many eukaryotic organisms. Besides its regulatory activity on SCF ubiquitination activity and some associated kinase activities little is known about single subunit functions. Here we present the characterization of the first and largest subunit of the COP9 signalosome, CsnA, in A. nidulans. A $\operatorname{csn} A$ deletion mutant displays a similar phenotype as described earlier for $\operatorname{csn} D$ and $\operatorname{csn} E$ deletions - the abundance of formation of mature sexual fruitbodies and red pigmentation of the mycelium on solid medium (Busch et $a l ., 2003)$. We were able to show that the mutant phenotypes of red pigmentation and inability to form cleistothecia are linked. Furthermore by expressing truncated CsnA proteins we were able to show that the N-terminal and the PCI domain of the protein have seperatable functions in the cells, the C-terminal part of the protein is essential for COP9 subunit interaction and sexual development in $A$. nidulans and the N-terminus for regulation of hyphal growth. Sexual fruitbody formation is directly or indirectly effected by a loss of deneddylation activity of the COP9 signalosome. 


\subsection{Introduction}

The COP9 signalosome is a conserved protein complex found in eukaryotes, though it could be shown, that not in every eukaryote all eight subunits are present. The COP9 signalosome was found in Arabidopsis thaliana as a regulator of light response (Wei et al., 1994). The mammalian COP9 complex was originally isolated as a co-purifying byproduct of the $26 \mathrm{~S}$ proteasome (Seeger et al., 1998). The complete isolation of subnunits of the COP9 complex from animals and plants revealed the evolutionary conservation of the protein complex. The COP9 signalosome resembles the regulatory $19 \mathrm{~S}$ subunit of the proteasome and the eIF3. The complex of higher eukaryotes consists of eight subunits CSN1 to CSN8 with decreasing molecular weight (Deng et al., 2000). Whereas $S$. pombe was only known to show six of the eight subunits and S. cerevisiae only a COP9 like complex with CSN5 being the only highly similar protein amongst the potentially identified subunits (Mundt et al., 1999; Mundt et al., 2002; Wee et al., 2002; Zhou et al., 2001). Six of the subunits form the core complex and harbor so-called PCI domains, which are found in the proteasome, $\underline{\mathrm{CSN}}$ and $\underline{\mathrm{IF}} 3$, in their C-

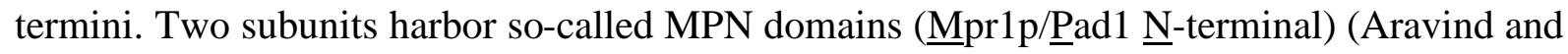
Ponting, 1998; Hofmann and Bucher, 1998; Ponting et al., 1999). In general the three complexes are referred to as PCI complexes.

The best studied intrinsic function of the COP9 signalosome is its deneddylation activity. Rub1 or Nedd8 is a small ubiquitin-like protein which is used by the cell to control the ubiquitin-dependent degradation of proteins through the SCF complex (Lyapina et al., 2001). Similar to ubiquitin ligation the conjugation of Nedd8 requires the Nedd8 activating enzymes APP-BP1 and Uba3 (E1) and the conjugating enzyme Ubc12 (E2) (Amir et al., 2002). The neddylation pathway is essential in S. pombe, C. elegans and mice and plays a significant role in plant auxin responses (del Pozo et al., 2002; Osaka et al., 2000; Tateishi et al., 2001). The SCF represents a E3 type ubiquitin ligase that catalyzes the third step in the substrate specific ubiquitin conjugation of target proteins. The COP9 signalosome complex binds to the SCF complex through CSN2, CSN6 and CSN1's N-terminus and deconjugates Nedd8 from the cullin subunit of the SCF through a metalloprotease activity assigned to CSN5 (Lyapina et al., 2001; Schwechheimer et al., 2001; Wang et al., 2002; Yang et al., 2002). CSN5 contains a metalloprotease motif called a JAMM (Jab1/MPN domain-associated metalloisopeptdase) (Ambroggio et al., 2004). The highly dynamic neddylation/deneddylation status of the cullin 
regulates the activity of ubiquitin conjugation to target proteins of the SCF complex (Yang et al., 2002; Zheng et al., 2002).

Recent investigations showed a common structural scaffold for PCI domains, which display relatively high interspecies divergence and vary highly in their degree in conservation between single PCI domain proteins (Scheel and Hofmann, 2005). Especially the N-terminal boundary of PCI domains is often difficult to determine. In silico investigations revealed that a PCI domain actually consists of two subdomains. The C-terminal half-domain is a globular $\alpha / \beta$ structure with a " $\alpha \beta \alpha \alpha \beta \beta$ " arrangement and can be classified as a winged helix fold or WH domain. The N-terminal half of the PCI domain is entirely helical and consists of three antiparallel hairpins that connect six regularly spaced helices. This half-domain resembles in a superhelix which is also found in HEAT and Armadillo (ARM) repeats and is thus called a HAM domain. ARM and HEAT motifs are tandemly repeated sequences of approximately 50 amino acid residues that occur in a wide variety of eukaryotic proteins (Andrade et al., 2001). In some PCI proteins a TPR-like repeat can be found to extend the HAM domain towards the N-terminus of the protein (Scheel and Hofmann, 2005).

The largest CSN subunit CSN1 has an essential role in complex assembly. A complete loss of this subunit in A. thaliana leads to accumulation of CSN8 and a decrease in CSN4 and CSN7 protein level, furtheron the fifth subunit dissociates from the complex, resulting in the inability to deneddylate its substrates (Wang et al., 2002). The N-terminal part of CSN1 is insufficient to incorporate into the complex, whereas the central domain and the C-terminus which harbors the PCI domain is sufficient to incorporate (Tsuge et al., 2001; Wang et al., 2002). The restoration of the complex by complementing the fus 6 mutant in $A$. thaliana with either a truncated protein harbouring parts of the PCI domain or the full PCI domain restores the Rub1 deconjugation or deneddylation activity of the complex (Wang et $a l .$, 2002). Recent studies revealed that one of at least three associated kinase activities directly interacts with CSN1 (Sun et al., 2002). The kinase is a inositol 1,3,4-triphosphate 5/6 kinase with a relatively wide substrate spectrum. It is able to phosphorylate c-Jun, IkBa, ATF2 and p53 and is inhibited by curcumin. This leads to the hypothesis that CNS regulates p53 in a highly subtle manner. CSN-mediated phosphorylation leads to p53 degradation in the 26S proteasome but CSN-mediated deneddylation may stabilize c-Jun and thus indirectly activate AP-1 (Sun et al., 2002; Wilson et al., 2001).

For the A. nidulans genome we could show that all eight subunits of the COP9 signalosome are represented by homologous genes (unpublished results). Deletion of the fourth and fifth subunit $\operatorname{csn} D$ and $\operatorname{csn} E$ lead to pleiotrophic phenotypes in A. nidulans. Fungal strains deleted 
for either of the subunit encoding genes are in contrast to higher eukaryotes, which are embryonal lethal, viable but produce shortened cells harboring a so far unknown red pigment after reaching developmental competence. Additionally, csn deletions result in a block in sexual fruitbody formation at the stage of primordia (Busch, et. al., 2003).

In this work we focus on the first and largest subunit of the COP9 signalosome, CsnA, in A. nidulans development. CsnA corresponds to CSN1 and follows the gene/protein nomenclature of $A$. nidulans proposed by the Aspergillus genome annotation consortium (Galagan et al., (submitted)). We analyzed the phenotypes generated by a loss of this single subunit and hence whole complex functions and parts of it in A. nidulans development. 


\subsection{Material and methods}

\subsubsection{Strains, media and growth conditions}

Strains of A.nidulans were cultivated at $37^{\circ} \mathrm{C}$ with minimal medium supplemented as described earlier (Bennett and Lasure, 1991; Käfer, 1977). Developmental cultures were synchronized by obtaining vegetative mycelium from submerged liquid culture after 18 hours to reach sexual competence and then transfer to solid medium. Sexual development was induced by cultivating strains in the dark under oxygen limitation, asexual development was induced by continous white light. Developmental cultures were synchronized by cultivating them in liquid culture for 18 hours to reach sexual competence and then transfer to solid medium. Expression from the alcA promoter was induced by adding $2 \%$ ethanol and $2 \%$ glycerol instead of glucose to the medium (Felenbok, 1991). In $\operatorname{csnA}$ deletion strain AGB223 the $\operatorname{csn} A$ gene was replaced by a $\operatorname{pyr} G$ cassette with flanking zeocin-resistance repeats. Counterselecting against the $p y r G$ markergene the marker was rescued due to mitotic recombination (Krappmann and Braus, 2003). Microscopy was performed by differential interface contrast (DIC).

\subsubsection{Molecular methods}

E. coli (Inoue et al., 1990) and A. nidulans (Eckert et al., 2000) transformations were carried out as described. Genomic DNAs of A. nidulans were isolated as described by Lee and Taylor, 1990. Standard techniques were applied for Southern analysis (Rave et al., 1979; Southern, 1975). DNAs for probes were obtained by digesting pME2937 with BamHI producing an approximately $1.5 \mathrm{~kb}$ fragment. DNA sequencing was carried out with an ABI310 genetic analyzer. Further sequence analysis was carried out with the Lasergene software from DNASTAR (DNASTAR, Madison, WI.). The nucleotide sequence of $\operatorname{csn} A$ was deposited in the GenBank database under accession Number AY574249. 


\subsubsection{Isolation of genomic and cDNA of $A$. nidulans}

The $\operatorname{csn} A$ gene was isolated by colony hybridisation of a genomic SalI sublibrary of A. nidulans and subcloned into pBluescript SK(+) (Stratagene) revealing plasmid pME2499. For colony hybridisation (Moseley et al., 1980) a probe obtained with primers SB104 and SB105 was used producing a 750bp DNA fragment. cDNA was obtained by colony hybridisation of a cDNA library and revealed plasmid pME2498. For the Southern experiment (Southern, 1975) revealing which chromosome carries the $\operatorname{csn} A$ gene the BAC library of $A$. nidulans obtained from Clemson University was used.

\subsubsection{Construction of plasmids for $A$. nidulans manipulation}

Deletion constructs were obtained according to Krappman et al., 2002. The 5'-flanking region was amplified by primers Elke19 5'ATC CCC GAA TAT TCC ACG CTG-3` and Elke20 5'-CGT CTA TGC TGG ACA GCT CG-3`. The 3'-flanking region was obtained by PCR with primers $c s n A-K O 35$-CCG TCG GAT ACT ACG TTT GGC-3` and $c \sin A-\mathrm{KO} 45^{`}$-AAC GAT CTC TCC ACC GGG ATG-3'. The 5'-region and the 3'-region were integrated into pME2409 and in vivo recombination was carried out with the excised deletion cassette and pME2499 producing the deletion construct pME2505.

The constructs for paritial complementation of the $\operatorname{csn} A$ deletion mutant were obtained by linearizing plasmid pME1565 with SmaI and inserting the truncated versions of the $\operatorname{csnA}$ ORF into the vector. The truncated versions of the ORF were produced by amplification with KOD proofreading polymerase using the following primer pairs ODOL-csnA1 5'-AAA GGT ACC ATG GAG CCC ATG TTA CCA GAAGCG-3'and ODOL-csnA2 5'AAA CCC GGG TTA TTG CTT CAT CCC CGT CGC CC -3 'to produce the full-length ORF resulting in pME2937, ODOL-csnA1 and ODOL-csnA3 5'- AAA CCC GGG TTA CAT GGA GGC TAG GGC ACA GAG -3’to amplify a truncated ORF encoding only amino acids 1 to 270 producing pME2934, ODOL-csnA1 and ODOL-csnA4 5'- AAA CCC GGG TTA TTG TTC TTC CGG CTT CCC CCC $-3^{\prime}$ to amplify a truncated ORF encoding for amino acids 1 to 228 producing pME2936 and ODOL-csnA5 5'- AAA GGT AAC ATG CAA GCG AAA CAC CAG CCT AAG -3' and ODOL-csn2 to amplify a truncated ORF encoding for amino acids 229 to the UAA STOP codon to produce pME2937. START and STOP codons have been integrated into the primers where needed. 


\subsection{Results}

\subsubsection{The A. nidulans $\operatorname{csnA}$ gene encodes a PCI domain protein similar to subunit I of the COP9 signalosome}

The $\underline{\text { COP9 }}$ signalosome (CSN) is a multiprotein complex found in higher eukaryotes (Wei and Deng, 2003). Two subunits of the A. nidulans CSN have been characterized and deleted in A. nidulans (Busch et al., 2003). Deletions of the genes for subunit 4 (csnD) and 5 (csnE) lead to multiple pleiotropic phenotypes. A third subunit of the proposed AnCSN, $\operatorname{csn} A$, was found in the CEREON database (http://microbial.cereon.com) by comparison to the genes for CSN1 subunits of other eukaryotes. According to the unified COP9 signalosome nomenclature the gene was named $\operatorname{csn} A$, though another gene of this name was already described to be a chitosanase by Rodriguez et al., 2004 (Accession number AAR85471). A $750 \mathrm{bp}$ fragment of genomic $A$. nidulans DNA was amplified and used as probe to isolate a cosmid from the ordered cosmid library of A. nidulans and a cDNA from a cDNA library. Southern hybridisations with genomic A. nidulans DNA revealed a single copy of the $\operatorname{csn} A$ gene on chromosome 7. The 750bp PCR fragment served to produce a genomic 8kb SalI fragment containing an open reading frame (ORF) of 1497bp (Figure 5). A corresponding cDNA of 1923bp was isolated from a cDNA library containing a 298bp 5' flanking region and a 128bp 3' flanking region. Comparisons between the isolated cDNA and the genomic DNA revealed that the open reading frame is disrupted by three introns of $48 \mathrm{bp}, 59 \mathrm{bp}$ and 52 bp respectively at positions $+142,+235$ and +576 relative to the A of the START codon set at position +1 (Figure 5). The deduced peptide sequence had a length of 498 amino acids and a calculated protein mass of $55,7 \mathrm{kDa}$. The peptide sequence contains a putative PCI domain (proteasome, COP9, eIF) and displayed identities up to $36,2 \%$ (calculated over the full protein) to various other proteins described as first subunits of the COP9 signalosome and even up to $44,4 \%$ if only the respective PCI domains were compared (Figure 6). Notably the sequence identities to higher eukaryotes were higher than those to its fungal relative $S$. pombe. 
A
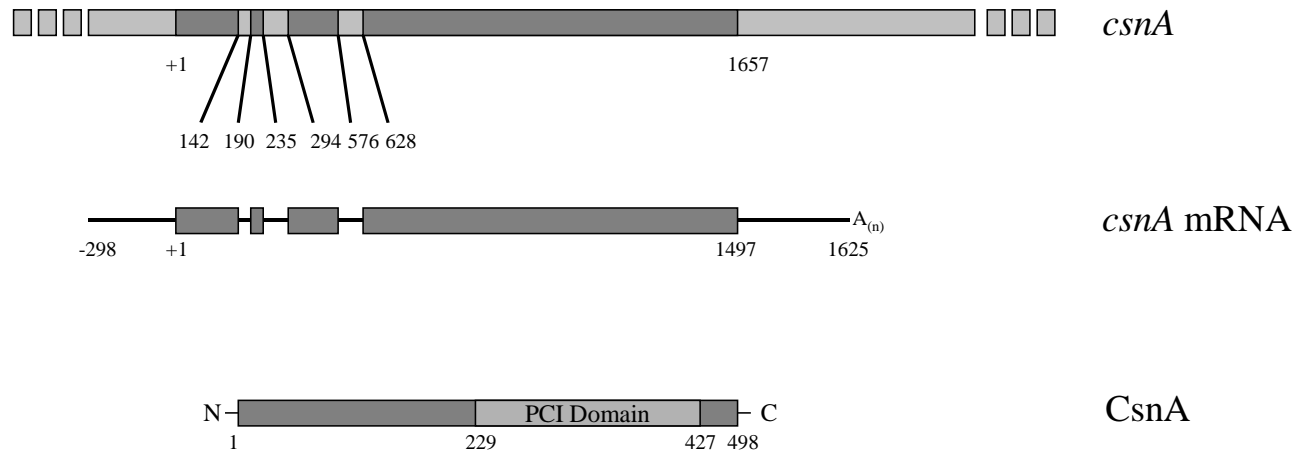

CsnA

B

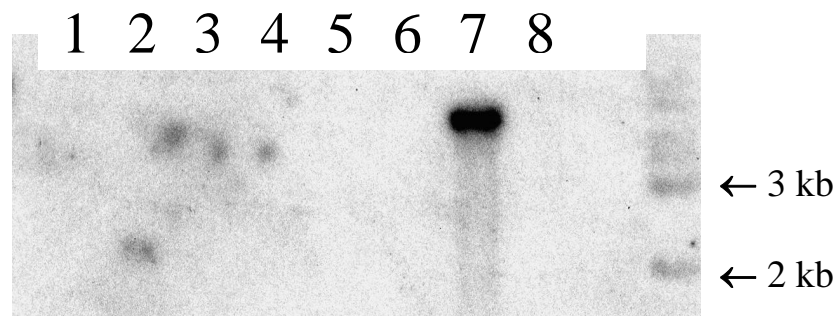

Fig. 5: The $\operatorname{csn} A$ gene of $A$. nidulans is located on chromosome 7

(A) The $\operatorname{csn} A$ gene locus (top), the resulting gene products (middle) and protein (bottom) are schematically displayed. The open reading frame starts at the A of the AUG start codon as position +1 and consists of 1497 bps. The ORF is interrupted by three introns at positions 142-190, 235-294 and 576-629 respectively. The isolated cDNA is shown as white bar and includes a $5^{\prime}$ and $3^{\prime}$ untranslated region of 298 bp and 128 bp respectively. The deduced CsnA protein consists of 498 amino acids with a calculated mass of 55,6 $\mathrm{kDa}$. The predicted $\mathrm{PCI}$ domain presumably necessary for protein-protein interaction is shown in grey.

(B) Southern hybridisations with genomic A. nidulans DNA revealed a single copy of the $\operatorname{csn} A$ gene on chromosome 7. 


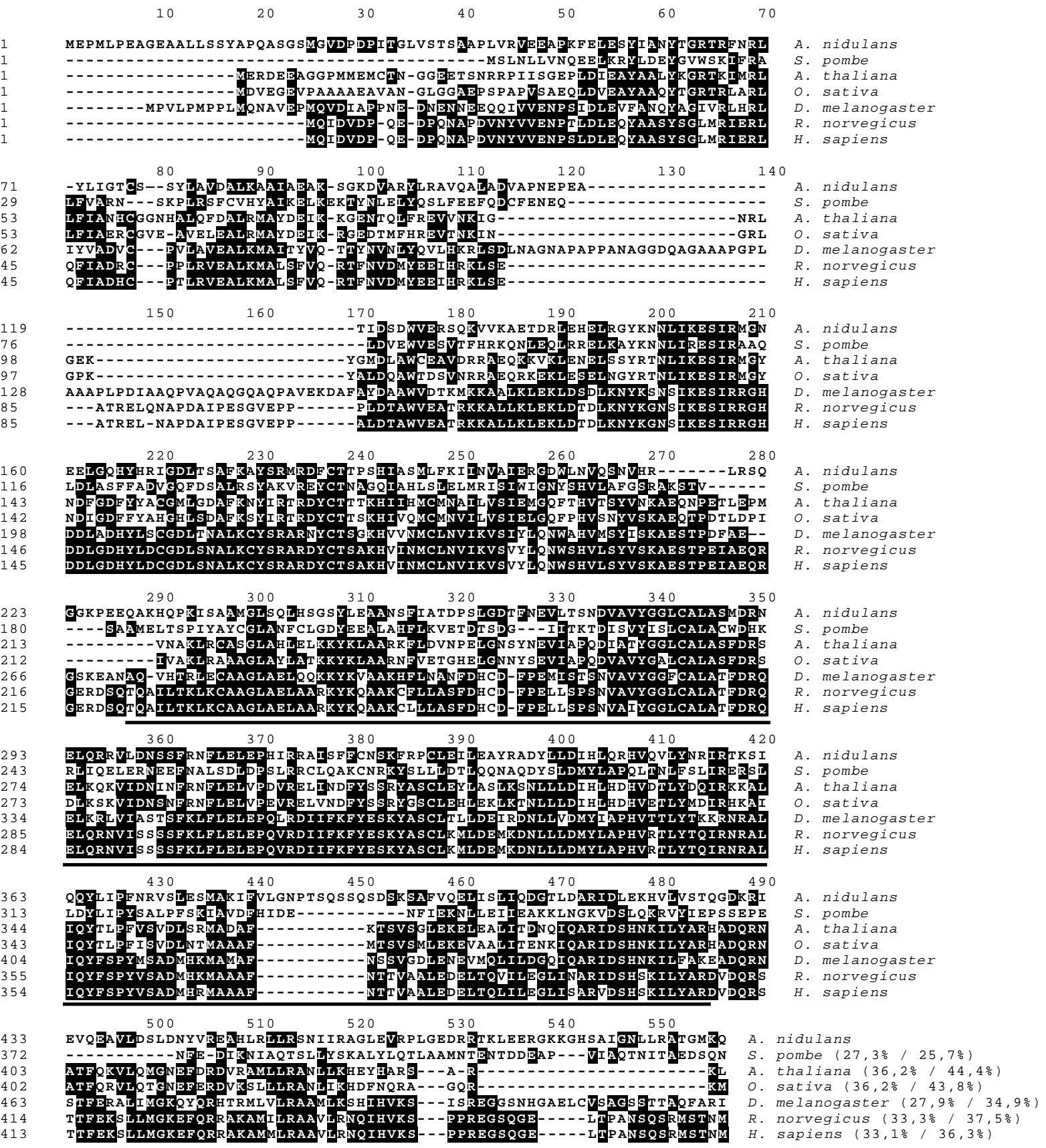

Fig. 6: CsnA of A. nidulans shows high amino acid identities to Csn1 proteins of higher eukaryotes

The multialignment of deduced amino acid sequences of CsnA (AY574249) to the corresponding sequences of $S$. pombe (O94308), A. thaliana (P45432), O. sativa (AAF40112), D. melanogaster (AAD28605), $R$. norvegicus (P97834) and H. sapiens (Q13098) is shown. Residues identical for all species are highlighted. The predicted conserved PCI domain presumably needed for interaction with other CSN subunits is underlined. Amino acid identities between CsnA and the Csn1 subunits of other organisms are displayed in brackets in the bottom row of the alignment. The first and second percentage numbers represent the identities of the full length protein, and the PCI domain respectively. 


\subsubsection{Aspergillus nidulans strains deleted for $\operatorname{csn} A$ are blocked in sexual development} and produce altered secondary metabolites

In A. nidulans strain AGB152 (a derivative of wild type strain A4 with the relevant genotype pyrG89) a $\operatorname{csn} A$ deletion was introduced, resulting in strain AGB223 $(\Delta \operatorname{csn} A / \operatorname{csn} A::(z e o R: p y r G: z e o))$ to compare the phenotype with $\Delta \operatorname{csn} D$ and $\Delta c s n E$ deletion strains which are blocked in sexual development (Busch et al., 2003). An overview of all A. nidulans strains with their COP9 related genotypes and associated phenotypes is presented in Figure 7. The $\operatorname{csn} A$ deletion strain AGB223 $\left(\mathrm{pyr}^{+}\right)$was treated with 5-fluorotic acid (5-FOA) to recycle the pyrG-marker of the deletion cassette (Krappmann and Braus, 2003) for further experiments resulting in strain AGB234 (pyrG $\left.{ }^{-}\right)$. A. nidulans strain AGB234 shows a similar phenotype to AGB223 on minimal medium containing uridine (Figure 8). When grown in liquid selective minimal medium no distinction from the wild type could be made regarding the growth behaviour. However, when grown on solid minimal medium for 48 hours, phenotypic anomalities become apparent. The $\Delta \operatorname{csn} A$ strain shows reduced cell size and red colouring (Figure 8). Equal to the other csn deletions of $A$. nidulans, the hyphae penetrate the agar and show a red pigmentation which is not observable in wild type strains. Sexual development in the $\Delta \operatorname{csn} A$ strain is arrested at the state of Hülle cells and primordia, but does not progress to the formation of fertile, mature sexual fruitbodies (see Figure 7). The growth behaviour of strain AGB223 and AGB234 respectively changes over time compared to wildtype strain AGB152. After approximately 10 passages of conidia harvest and growing of the newly harvested conidia on solid medium the strains continually produce less conidia and stop producing sexual structures at all (see Figure 8). 
Genotype

\begin{tabular}{|c|c|c|}
\hline AGB152 & & wild type \\
\hline AGB195 & $\Delta \operatorname{csn} D$ & red \\
\hline AGB209 & $\Delta c s n E$ & red \\
\hline AGB233 & $\Delta \operatorname{csn} A$ & red \\
\hline AGB234 & $\Delta c \operatorname{sn} A\left(p y r G^{-}\right)$ & red \\
\hline AGB240 & 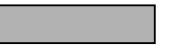 & red \\
\hline AGB235 & & red \\
\hline AGB236 & & wild type \\
\hline AGB237 & & wild type \\
\hline
\end{tabular}

Phenotype

Cleistothecia

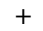

Fig. 7: Nomenclature and associated phenotypes of the investigated $A$. nidulans strains impaired in the COP9 signalosome in comparison to wildtype

This figure gives an overview of the investigated strains in this study and summarizes the phenotypes. Pigmentation of the hyphae is indicated as red for red pigmentation and as wild type for wild type pigmentation. The ability to form cleistothecia is indicated by plus, if the strains are not able to form fruitbodies, this is indicated by minus.

\subsubsection{Expression of truncated CsnA peptides can restore wild type secondary metabolism and sexual development in $A$. nidulans $\operatorname{csn} A$ deletion strains}

Aspergillus nidulans strains that lack either the CSN subunits CsnA, CsnD or CsnE exhibit pleiotropic mutant phenotypes of red pigmented hyphae during secondary metabolism and the disability to form fertile sexual fruitbodies. To assign a function to CsnA as part of the COP9 signalosome, we had to distinguish between phenotypes due to loss of assembly of the CSN complex caused by missing subunits and phenotypes that are caused by a loss of CsnA itself. To distinguish between these phenotypes and to investigate the role and function of CsnA in the multiprotein complex, truncated CsnA peptides were expressed in a $\operatorname{csn} A$ deletion strain. The truncated peptides were expressed from an alcA promoter (Felenbok, 1991) under 
repressive conditions to simulate low expression of the gene product and to avoid titration of CsnA complexing subunits, though overexpression of CSN1 subunits was not found to produce any effect in higher eukaryotes (Wang et al., 2002).
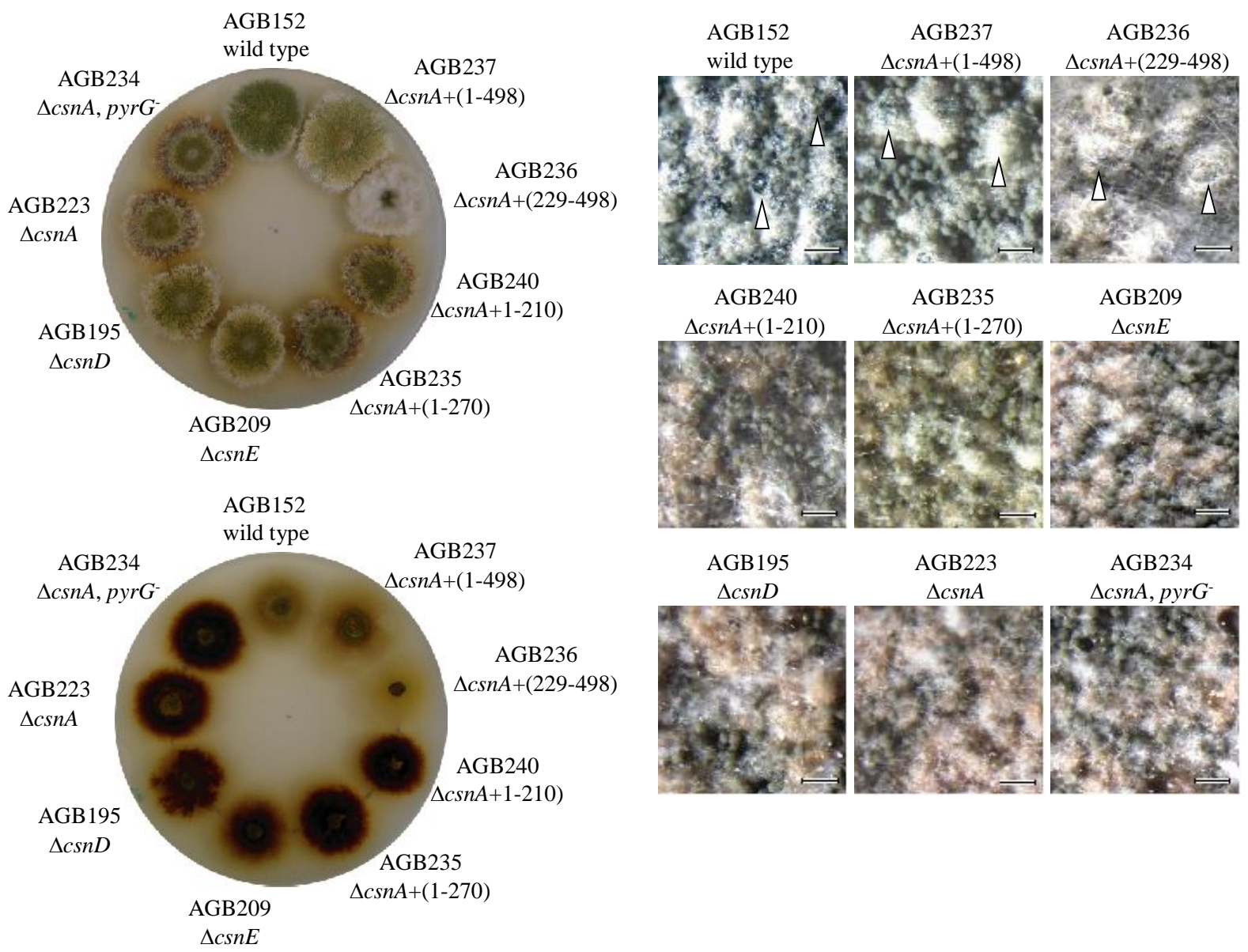

Fig. 8: Phenotypes of $A$. nidulans $\operatorname{csn} A$ mutant strains

Secondary metabolite and fruitbody (cleistothecia) formation of CSN deletion strains and partial complementations of $\operatorname{csnA}$ deletion mutant are shown. A. nidulans strains that lack CSN subunits exhibit the pleiotropic mutant phenotypes of red pigmented hyphae and the disablility to form fertile sexual fruitbodies after 96 hours of growth. A. nidulans strains AGB152 (wild-type), AGB223 $(\Delta \operatorname{csn} A)$, AGB234 ( $\Delta \operatorname{csn} A$ with restored $p y r G$-marker), AGB195 $(\Delta \operatorname{csn} D)$ and AGB209 $(\Delta \operatorname{csn} E)$, were grown on solid air-medium interface to allow development as control strains for AGB235 $(\Delta \operatorname{csn} A+$ (1-289)), AGB240 ( $\Delta \operatorname{csn} A+(1-228))$, AGB236 $(\Delta \operatorname{csn} A+(229-498))$ and AGB237 $(\Delta \operatorname{csn} A+(1-498))$. The left hand pictures show the overall colony phenotype of colonies grown for 96 hours on solid medium (top) to allow development and production of secondary metabolites, in this case the red pigment of the hyphae which becomes clearly visible after thorough washing off of the non-agarpenetrating parts of the colonies (bottom). The right hand pictures show a magnified view of the same colonies indicating cleistothecia when present by a white arrow. The black bar indicates a relative length of $200 \mu \mathrm{m}$. AGB223 and AGB243, the csn deletion strains, strain AGB235 and AGB240 display the above mentioned pleiotropic phenotypes. In the remaining strains the expression of parts of CsnA complements the pleiotropic phenotypes. 
As control a strain was constructed that expresses the full length protein from the alcA promoter to show differences between the complementation strain and the wild type. A. nidulans strains AGB152 (wild type), AGB195 ( $\Delta \operatorname{csn} D)$, AGB209 $(\Delta \operatorname{csn} E)$, AGB223 ( $\Delta \operatorname{csn} A)$ and AGB234 (AGB223 with restored pyrG-marker) were grown on solid air-medium interface to allow development in comparison to the strains AGB240 $(\Delta \operatorname{csn} A+(1-228))$, AGB235 $(\Delta \operatorname{csn} A+(1-289)), \operatorname{AGB} 236(\Delta \operatorname{csn} A+(229-498))$ and AGB237 $(\Delta \operatorname{csn} A+(1-498))$ expressing truncated versions of CsnA. In Figure 8 the overall colony phenotype of the strains is displayed after 96 hours of growth, allowing sexual differentiation and induction of secondary metabolism.

To show the altered pigmentation of the mutant strains, the plates were thoroughly washed after incubation and excess mycelium was removed from the agar surface to depict pigmented hyphae which have penetrated the agar. The wild type forms mature cleistothecia and the underground of the colony is not colored after 96 hours of growth. The complementation strain AGB237 expressing the csnA-cDNA behind an alcA promoter in strain AGB234 restores all pleiotropic phenotypes typical for $c s n$ deletion mutants. The $\operatorname{csn} A, \operatorname{csn} D$ and $c \operatorname{sn} E$ deletion strains of $A$. nidulans are unable to form cleistothecia and stop sexual development at the stage of primordia. The underground of the colonies is reddish-brown due to pigmentation of the agar penetrating hyphae. In strain AGB240 only the N-terminus of CsnA is expressed lacking the central domain and the PCI domain of the protein. This strain displays the same phenotype as the $\operatorname{csnA}$ deletion strain AGB234. Strain AGB235 expresses the $\mathrm{N}$-terminus and parts of the PCI domain of CsnA, its phenotype is similar to the investigated csn deletion strains. In the right column all investigated strains are displayed in a magnified view. In strain AGB236 the PCI domain of the protein and the central domain are expressed. The strain is able to form mature cleistothecia and lacks the typical red pigmentation of the deletion strain. The top of the colony is covered with a thick layer of aerial hyphe that covers the mass of conidia and nests with mature cleistothecia. These results suggest a correlation of a defect in sexual development and formation of red pigmented hyphae in $\operatorname{csn}$ mutant strains. On the other hand the PCI domain of CsnA alone is sufficient to restore the pleiotropic effects seen in a $\operatorname{csn} A$ null mutant. 


\subsubsection{COP9 mutants display a growth defect in vegetative mycelium on solid medium at $37^{\circ} \mathrm{C}$}

The radial growth of all investigated strains was measured at $30^{\circ} \mathrm{C}$ and $37^{\circ} \mathrm{C}$ and compared to the wild type to investigate the growth rate of the csn mutant strains and complemented strains. We could show that the growth rate of all investigated mutants is similar to the growth rate of the wild type strain at $30^{\circ} \mathrm{C}$. Interestingly when grown at $37^{\circ} \mathrm{C}$ the growth rate of the wild type strain increased, whereas the growth rate of the mutant strains and of strain AGB237 expressing the full-length csnA-cDNA and AGB236 encoding the PCI domain of CsnA does not increase (Figure 9). We could show that the red hyphae phenotype and the acleistothecial phenotype are linked and can be complemented by CsnA in a csnA deletion strain. The results of the growth tests indicate that the exact amount of CsnA seems to be important to restore a wild type growth phenotype, since the expression of the $\operatorname{csn} A$-cDNA from the alcA promoter does not complement the growth phenotype at $37^{\circ} \mathrm{C}$ but only the formation of cleistothecia and abolishes formation of red hyphae. 


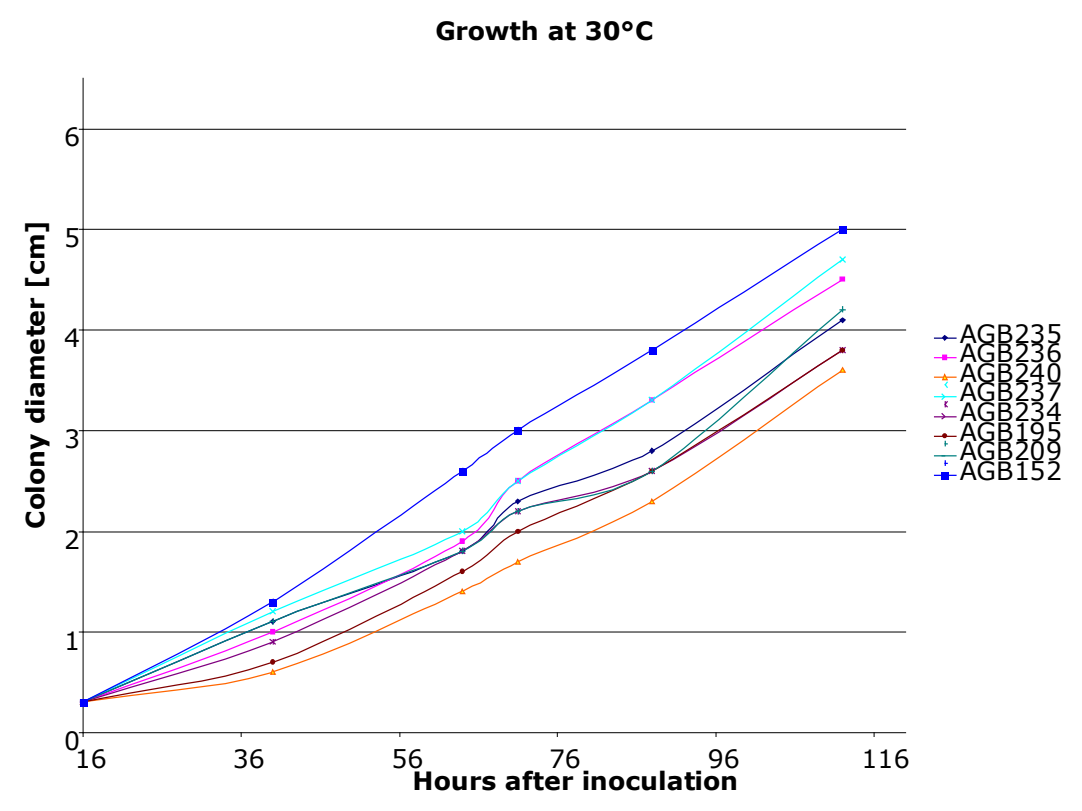

Growth at $37^{\circ} \mathrm{C}$

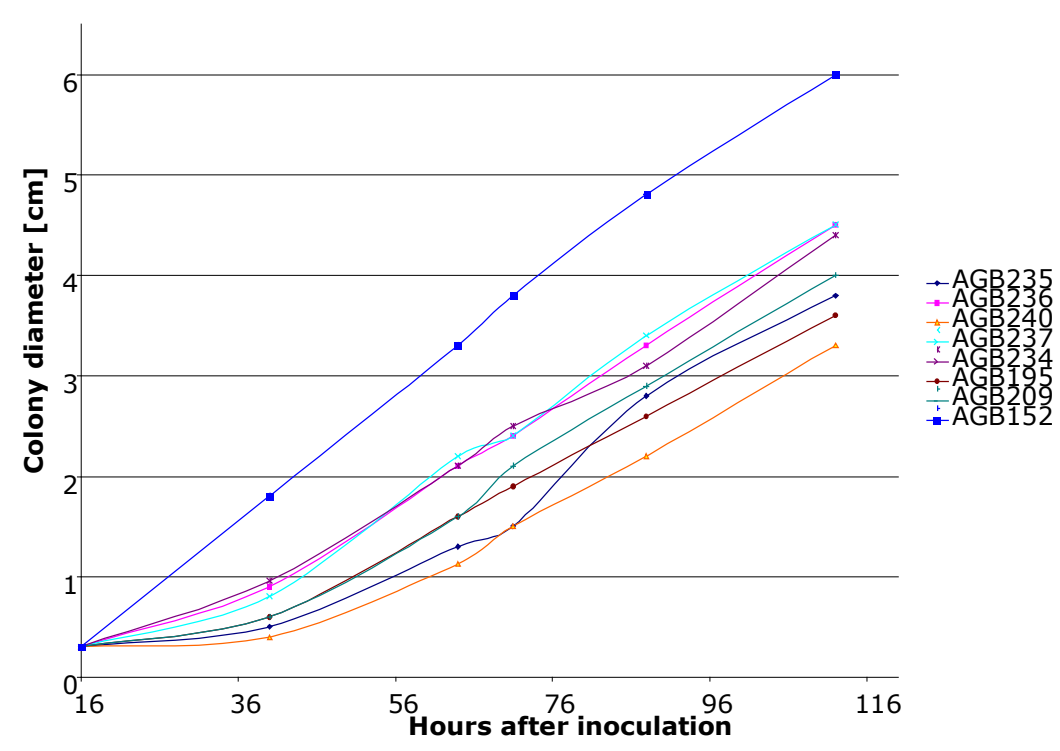

Fig. 9: Growth curves of the $A$. nidulans strains expressing full or truncated $\operatorname{csn} A$ genes at $30^{\circ} \mathrm{C}$ and $37^{\circ} \mathrm{C}$

The colony diameter at given time points after inoculation was determined by measuring 3 independent colonies of the respective strains. The growth rate at $30^{\circ} \mathrm{C}$ and $37^{\circ} \mathrm{C}$ is shown. Whereas at $30^{\circ} \mathrm{C}$ all investigated strains grow with approximately the same growth rate at $37^{\circ} \mathrm{C}$ only the wild type strain $\mathrm{AGB} 152$ is able to increase its growth rate. The full and partially complemented strains, as well as the $\operatorname{csn}$ deletion strains, are unable to grow faster at $37^{\circ} \mathrm{C}$. 
2.4.5 Structural in silico analysis of the investigated truncated CsnA proteins of A. nidulans

In eukaryotic organisms at least three distinct multi-protein complexes exist, that are referred to as PCI complexes which display a similar subunit architecture despite their different function: the $26 \mathrm{~S}$ proteasome lid, the eukaryotic translation initiation factor eIF3 and the COP9 signalosome. These three multi-protein complexes have subunits harboring PCI domains and MPN domains in common. The degree of conservation between PCI subunits is highly variable. Apart from the similarities between proteasome subunits and subunits of the COP9 signalosome, which are relatively easy to identify, the similarities between other paralogous PCI subunits are hard to detect by simple sequence comparison. A bioinformatic approach (Scheel and Hofmann, 2005) predicts that the PCI domain is not necessarily a single domain in a structural sense but rather consists of two subdomains: a C-terminal winged helix domain WH domain with a key role in PCI:PCI interaction and a preceeding helical repeat region consisting of HEAT or Armadillo-like repeats (HAM domain). A TPR-like repeat (tetratrico-peptide repeat) region N-terminal of the HAM domain seems most likely to form an uninterrupted extension of the HAM domain. Interestingly this prediction was made on the base of secondary structure prediction and not on sequence similarity and seems to be the case for all the investigated PCI domain proteins. We adapted the structure prediction of Scheel et $a l$. to our experiments in expressing truncated CsnA proteins in A. nidulans by aligning CsnA to the investigated highly similar hsCSN1 and transferring the secondary structure to CsnA to investigate which regions of the CsnA protein are still present in our truncated proteins. We deduced that in strain AGB240 only the N-terminal TPR-like repeats are expressed, whereas in strain AGB235 the TPR-like repeats and three of six helices of the HAM domain are expressed. In strain AGB236 the HAM domain and the WH domain are expressed and the protein lacks the TPR-like repeats of the full protein (Figure 10). We conclude that the WH and HAM domain are essential for complexation of the CSN, since our results show that these domains are sufficient to complement the acleistothecial and red hyphae phenotype. 
A

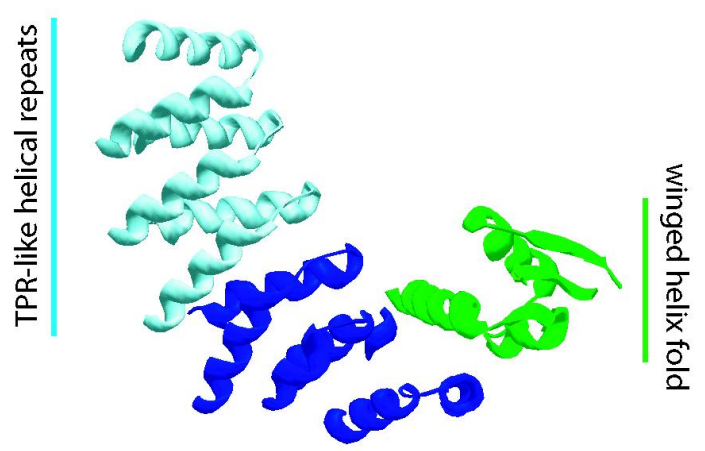

HAM domain

$\mathrm{PCl}$ domain

B

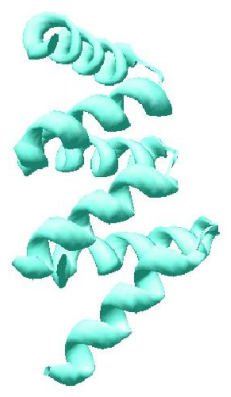

C

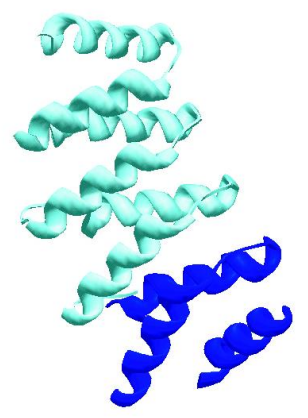

D

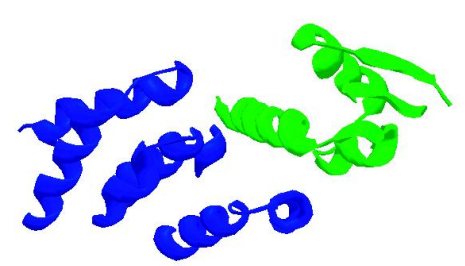

Fig. 10: Structure prediction of truncated versions of the CsnA protein according to Scheel and Hofmann

A predicted protein structure of the CsnA protein and its truncated versions are shown. The structural modelling was done according to Scheel et. al., 2005. (A) the bipartite PCI domain is indicated as a WH-fold (winged helix fold, green) and a HAM (HEAT/Armadillo repeats, dark blue) domain. The WH domain consists of three short $\beta$-strands which form an antiparallel sheet and three $\alpha$-helices in an " $\alpha \beta \alpha \alpha \beta \beta$ " arrangement, whereas the HAM-domain consists of $3 \alpha$-hairpins. The N-terminal TPR-repeat part (light blue) was assumed by Scheel and Hofmann as a general part of PCI domain proteins. (B) The truncated protein expressed in strain AGB240 is shown. It contains only the TPR-like repeats, but lacks all of the bipartite PCI domain. (C) The truncated protein expressed in strain AGB235 is shown, only the first and half of the second $\alpha$-hairpin of the HAM-domain remains. (D) The truncated CsnA protein is shown that is expressed in AGB236. The full PCI domain is expressed, but it lacks the TPR-like repeats. 


\subsection{Discussion}

We provide evidence that the $\operatorname{csn} A$ gene codes for the first and largest of eight subunits of the COP9 complex in A. nidulans. The protein, especially the PCI domain of the protein, shows high similarities to PCI domains of homologues of higher eukaryotes and slightly lower amino acid similarities to its counterpart of S. pombe. Comparing only the PCI domain of the protein to other CsnA homologues we find that the bipartite domain is significantly higher conserved than the rest of the protein. The fact that the CsnA subunit of the A. nidulans COP9 complex is higher conserved to higher eukaryotes like A. thaliana and $H$. sapiens in combination to the fact that other fungi like $S$. pombe contain only six of the eight subunits of the signalosome (Mundt et al., 1999) and S. cerevisiae forms only a COP9-like complex (Maytal-Kivity et al., 2002; Wee et al., 2002), with only subunit five subunits beeing conserved, leads us to the assumption that the complex plays a more significant role in the life and development of the filamentous fungus A. nidulans and serves as a more suitable model organism to investigate overall COP9 functions than the above named unicellular fungi.

The deletion of the $\operatorname{ssn} A$ gene leads to a similar pleiotropic phenotype that was formerly reported for deletions in the genes of subunits CsnD and CsnE (Busch et al., 2003). All so far characterized $c s n$ deletion mutants form distinct red hyphae that deeply penetrate the medium and are blocked in sexual development at the stage of primordia formation. Growth tests at $30^{\circ} \mathrm{C}$ and $37^{\circ} \mathrm{C}$ showed that $\operatorname{csn}$ deletion mutants display a slow growth phenotype at $37^{\circ} \mathrm{C}$ in contrast to the wild type. Where the wild type grows faster at $37^{\circ} \mathrm{C}$ than at $30^{\circ} \mathrm{C}$ the increase in growth rate can not be observed in csn deletion mutants. Partial or even full complementation of the $\operatorname{csn} A$ gene expressed under the alcA promoter did not restore this phenotype, though other observed phenotypes can be restored by partial or full complementation of the the $\operatorname{csn} A$ gene. We assume that expression of the gene under control of the alcA promoter may have an effect on the protein level of CsnA which leads to stochiometric discrepancies to other subunits of the COP9 signalosome and thus can not fully restore the wild type growth but is sufficient to restore the effects leading to the observed pleiotropic phenotypes.

CSN1 has an essential role in complex assembly. Complete loss of CSN1 in Arabidopsis abolishes accumulation of CSN8, CSN5 dissociates from the complex, and leads to a significant reduction in the levels of CSN4 and CSN7 (Wang et al., 2002). Plants and other higher eukaryotes as mammals and fly that lack the functionality of any subunit of the signalosome die during early embryogenesis (Freilich et al., 1999; Wei and Deng, 1992). In 
contrast to this the deletion of $\operatorname{csn}$ subunits in the filamentous fungus $A$. nidulans is not lethal for the whole organism but leads to the formation of a red pigment and a block in sexual development. The block of sexual development at the stage of primordia though, may well be regarded as embryonal lethality. So far we were unable to separate these two phenotypes. We assume that the loss of single subunits leads to either the complete loss of the complex in A. nidulans and to the loss of COP9 signalosome specific functions which are necessary to abolish the production of the red metabolite and drive the sexual development past the point of primordia formation. Interestingly the introduction of loss of function point mutations into the conserved JAMM domain of CsnE leads to similar phenotypes as reported for deletion mutants in any of the subunits (K. Nahlik, unpublished results). This hypothesis is strengthened by the fact that the expression of the C-terminal domain containing the PCI domain of CsnA, which is important for complex formation in plants (Tsuge et al., 2001; Wang et al., 2002), in strain AGB236 is sufficient to complete sexual development and abolish the production of the red hyphae phenotype. We deduce that the expression of the PCI domain alone and thus the proper formation of the CSN complex is essential for sexual development in A. nidulans. The HAM part of the PCI domain and the N-terminal domain of the CsnA protein are insufficient to restore sexual development and abolish red pigment formation, as proven in strain AGB235. Thus we could show that both parts of the bipartite PCI domain, the N-terminal HAM domain and the C-terminal WH domain are essential for formation of the complex and to restore specific complex functions.

It could be shown in plant that the inability to form the COP9 complex abolishes the deneddlyation activity of the COP9 complex due to dissociation of the metalloprotease subunit CSN5 (Wang et al., 2002). The inability to deneddylate and thus regulate the SCF complex activity leads to early embryonic death in higher eukaryotes which we can compare to an early embryonic death of A. nidulans progeny at the stage of primordia. Again, this is strengthened by the fact that mutants defective in deneddylation exhibit a similar phenotype (K. Nahlik, unpublished results). On the other hand the expression of the C-terminus including the PCI domain of CsnA is insufficient to fully restore a wild type phenotype. The lack of the $\mathrm{N}$-terminus of the CsnA protein leads to the formation of excessive aerial hyphae in the filamentous fungus A. nidulans, whereas the expression of the $\mathrm{N}$-terminus alone or the expression of the N-terminus and the HAM domain seem to suppress this phenotype. In Arabidopsis mutants that lack the N-terminus of CSN1 are not viable despite the fact that the COP9 complex and deneddylation activity are restored (Wang et al., 2002), whereas $\Delta c s n A$ mutants of $A$. nidualns expressing the $\mathrm{C}$-terminus only are able to produce mature sexual 
fruitbodies. This leads us to the conclusion that the inability to deneddylate due to a loss of the COP9 complex has a direct or indirect effect on sexual development in A. nidulans. So far one can only speculate if the $\mathrm{N}$-termius is additionally required for the deneddylation activity of the complex or if a direct interaction of the N-terminus with the Rbx1 subunit of the SCF complex affects other aspects of SCF activity (Wang et al., 2003) and thus viability or in this case the repression of production of excessive aerial hyphae.

In summary, we have shown that the loss of the largest subunit of the A. nidulans CSN leads to either the loss of the whole complex or at least to the loss of specific CSN functions. Most likely the loss of the deneddylation activity results in acleistothecial strains producing red hyphae. Additionally the exact amount of CsnA protein seems to be important for growth at higher temperatures. Separating CSN complex function and activities from CsnA activities, our results indicate a direct or associated activity in the N-terminal domain of CsnA. 


\subsection{References}

Ambroggio, X.I., Rees, D.C., and Deshaies, R.J. (2004) JAMM: A Metalloprotease-Like Zinc Site in the Proteasome and Signalosome. PLoS Biol 2: E2.

Amir, R.E., Iwai, K., and Ciechanover, A. (2002) The NEDD8 pathway is essential for SCF(beta -TrCP)mediated ubiquitination and processing of the NF-kappa B precursor p105. J Biol Chem 277: 2325323259.

Andrade, M.A., Petosa, C., O'Donoghue, S.I., Muller, C.W., and Bork, P. (2001) Comparison of ARM and HEAT protein repeats. J Mol Biol 309: 1-18.

Aravind, L., and Ponting, C.P. (1998) Homologues of 26S proteasome subunits are regulators of transcription and translation. Protein Sci 7: 1250-1254.

Bennett, J.W., and Lasure, L.L. (1991) Growth media. In More gene manipulation in fungi. Bennett, J.W. and Lasure, L.L. (eds). San Diego: Academic Press, pp. 441-457.

Busch, S., Eckert, S.E., Krappmann, S., and Braus, G.H. (2003) The COP9 signalosome is an essential regulator of development in the filamentous fungus Aspergillus nidulans. Mol Microbiol 49: 717-730.

del Pozo, J.C., Dharmasiri, S., Hellmann, H., Walker, L., Gray, W.M., and Estelle, M. (2002) AXR1-ECR1dependent conjugation of RUB1 to the Arabidopsis Cullin AtCUL1 is required for auxin response. Plant Cell 14: 421-433.

Deng, X.W., Dubiel, W., Wei, N., Hofmann, K., and Mundt, K. (2000) Unified nomenclature for the COP9 signalosome and its subunits: an essential regulator of development. Trends Genet 16: 289.

Eckert, S.E., Kübler, E., Hoffmann, B., and Braus, G.H. (2000) The tryptophan synthase-encoding $\operatorname{trpB}$ gene of Aspergillus nidulans is regulated by the cross-pathway control system. Mol Gen Genet 263: 867-876.

Felenbok, B. (1991) The ethanol utilization regulon of Aspergillus nidulans: the alcA-alcR system as a tool for the expression of recombinant proteins. J Biotechnol 17: 11-17.

Freilich, S., Oron, E., Kapp, Y., Nevo-Caspi, Y., Orgad, S., Segal, D., and Chamovitz, D.A. (1999) The COP9 signalosome is essential for development of Drosophila melanogaster. Curr Biol 9: 1187-1190.

Galagan, J.E., Calvo, S.E., Cuomo, C., Ma, L., Wortman, J., Batzoglou, S., Lee, S., Brudno, M., Bastürkmen, M., Spevak, C.C., Clutterbuck, J., Kapitonov, V., J., J., Scazzocchio, C., Farman, M., Butler, J., Purcell, S., Harris, S., Braus, G.H., Draht, O., Busch, S., D`Enfert, C., Bouchier, C., Goldman, G.H., GriffithsJones, S., Vienken, K., Pain, A., Selker, E.U., Archer, D., Penalva, M.Á., Oakley, B.R., Momany, M., Sano, M., Tanaka, T., Kumagai, T., Machida, M., Nierman, W.C., Denning, D.W., Caddick, M., Hynes, M., Paoletti, M., Fischer, E.R., Miller, J.B., Dyer, P., Sachs, M.S., Osmani, S.A., and Birren, B. (2005) Sequencing of Aspergillus nidulans and comparative analysis with A. fumigatus and A. oryzae. Manuscript submitted.

Hofmann, K., and Bucher, P. (1998) The PCI domain: a common theme in three multiprotein complexes. Trends Biochem Sci 23: 204-205.

Inoue, H., Nojima, H., and Okayama, H. (1990) High efficiency transformation of Escherichia coli with plasmids. Gene 96: 23-28. 
Käfer, E. (1977) The anthranilate synthetase enzyme complex and the trifunctional trpC gene of Aspergillus. Can J Genet Cytol 19: 723-738.

Krappmann, S., and Braus, G.H. (2003) Deletion of Aspergillus nidulans aroC using a novel blaster module that combines ET cloning and marker rescue. Mol Genet Genomics 268: 675-683.

Lyapina, S., Cope, G., Shevchenko, A., Serino, G., Tsuge, T., Zhou, C., Wolf, D.A., Wei, N., and Deshaies, R.J. (2001) Promotion of NEDD-CUL1 conjugate cleavage by COP9 signalosome. Science 292: 1382-1385.

Maytal-Kivity, V., Piran, R., Pick, E., Hofmann, K., and Glickman, M.H. (2002) COP9 signalosome components play a role in the mating pheromone response of $S$. cerevisiae. EMBO Rep 3: 1215-1221.

Moseley, S.L., Huq, I., Alim, A.R., So, M., Samadpour-Motalebi, M., and Falkow, S. (1980) Detection of enterotoxigenic Escherichia coli by DNA colony hybridization. J Infect Dis 142: 892-898.

Mundt, K.E., Porte, J., Murray, J.M., Brikos, C., Christensen, P.U., Caspari, T., Hagan, I.M., Millar, J.B., Simanis, V., Hofmann, K., and Carr, A.M. (1999) The COP9/signalosome complex is conserved in fission yeast and has a role in S phase. Curr Biol 9: 1427-1430.

Mundt, K.E., Liu, C., and Carr, A.M. (2002) Deletion mutants in COP9/signalosome subunits in fission yeast Schizosaccharomyces pombe display distinct phenotypes. Mol Biol Cell 13: 493-502.

Osaka, F., Saeki, M., Katayama, S., Aida, N., Toh, E.A., Kominami, K., Toda, T., Suzuki, T., Chiba, T., Tanaka, K., and Kato, S. (2000) Covalent modifier NEDD8 is essential for SCF ubiquitin-ligase in fission yeast. Embo J 19: 3475-3484.

Ponting, C.P., Aravind, L., Schultz, J., Bork, P., and Koonin, E.V. (1999) Eukaryotic signalling domain homologues in archaea and bacteria. Ancient ancestry and horizontal gene transfer. J Mol Biol 289: 729745 .

Rave, N., Crkvenjakov, R., and Boedtker, H. (1979) Identification of procollagen mRNAs transferred to diazobenzyloxymethyl paper from formaldehyde agarose gels. Nucleic Acids Res 6: 3559-3567.

Scheel, H., and Hofmann, K. (2005) Prediction of a common structural scaffold for proteasome lid, COP9signalosome and eIF3 complexes. BMC Bioinformatics 6: 71.

Schwechheimer, C., Serino, G., Callis, J., Crosby, W.L., Lyapina, S., Deshaies, R.J., Gray, W.M., Estelle, M., and Deng, X.W. (2001) Interactions of the COP9 signalosome with the E3 ubiquitin ligase SCFTIRI in mediating auxin response. Science 292: 1379-1382.

Seeger, M., Kraft, R., Ferrell, K., Bech-Otschir, D., Dumdey, R., Schade, R., Gordon, C., Naumann, M., and Dubiel, W. (1998) A novel protein complex involved in signal transduction possessing similarities to 26S proteasome subunits. Faseb J 12: 469-478.

Southern, E.M. (1975) Detection of specific sequences among DNA fragments separated by gel electrophoresis. J Mol Biol 98: 503-517.

Sun, Y., Wilson, M.P., and Majerus, P.W. (2002) Inositol 1,3,4-trisphosphate 5/6-kinase associates with the COP9 signalosome by binding to CSN1. J Biol Chem 277: 45759-45764.

Tateishi, K., Omata, M., Tanaka, K., and Chiba, T. (2001) The NEDD8 system is essential for cell cycle progression and morphogenetic pathway in mice. J Cell Biol 155: 571-579. 
Tsuge, T., Matsui, M., and Wei, N. (2001) The subunit 1 of the COP9 signalosome suppresses gene expression through its N-terminal domain and incorporates into the complex through the PCI domain. J Mol Biol 305: $1-9$.

Wang, X., Kang, D., Feng, S., Serino, G., Schwechheimer, C., and Wei, N. (2002) CSN1 N-terminal-dependent activity is required for Arabidopsis development but not for Rub1/Nedd8 deconjugation of cullins: a structure-function study of CSN1 subunit of COP9 signalosome. Mol Biol Cell 13: 646-655.

Wang, X., Feng, S., Nakayama, N., Crosby, W.L., Irish, V., Deng, X.W., and Wei, N. (2003) The COP9 signalosome interacts with SCF UFO and participates in Arabidopsis flower development. Plant Cell 15: 1071-1082.

Wee, S., Hetfeld, B., Dubiel, W., and Wolf, D.A. (2002) Conservation of the COP9/signalosome in budding yeast. BMC Genet 3: 15 .

Wei, N., and Deng, X.W. (1992) COP9: a new genetic locus involved in light-regulated development and gene expression in Arabidopsis. Plant Cell 4: 1507-1518.

Wei, N., Chamovitz, D.A., and Deng, X.W. (1994) Arabidopsis COP9 is a component of a novel signaling complex mediating light control of development. Cell 78: 117-124.

Wei, N., and Deng, X.W. (2003) The COP9 signalosome. Annu Rev Cell Dev Biol 19: 261-286.

Wilson, M.P., Sun, Y., Cao, L., and Majerus, P.W. (2001) Inositol 1,3,4-trisphosphate 5/6-kinase is a protein kinase that phosphorylates the transcription factors c-Jun and ATF-2. J Biol Chem 276: 40998-41004.

Yang, X., Menon, S., Lykke-Andersen, K., Tsuge, T., Di, X., Wang, X., Rodriguez-Suarez, R.J., Zhang, H., and Wei, N. (2002) The COP9 signalosome inhibits p27(kip1) degradation and impedes G1-S phase progression via deneddylation of SCF Cul1. Curr Biol 12: 667-672.

Zheng, J., Yang, X., Harrell, J.M., Ryzhikov, S., Shim, E.H., Lykke-Andersen, K., Wei, N., Sun, H., Kobayashi, R., and Zhang, H. (2002) CAND1 binds to unneddylated CUL1 and regulates the formation of SCF ubiquitin E3 ligase complex. Mol Cell 10: 1519-1526.

Zhou, C., Seibert, V., Geyer, R., Rhee, E., Lyapina, S., Cope, G., Deshaies, R.J., and Wolf, D.A. (2001) The fission yeast $\mathrm{COP} 9 /$ signalosome is involved in cullin modification by ubiquitin-related Ned8p. BMC Biochem 2: 7. 


\section{Chapter 3}

Annotation of cross-pathway control related genes in the genome of Aspergillus nidulans, Aspergillus fumigatus and Aspergillus oryzae

\subsection{Abstract}

Amino acids are essential building blocks of protein biosynthesis. The regulatory network dealing with the uptake of amino acids, the synthesis of amino acids from precursors and their channeling into protein production is complex. A comparative genome analysis of the model organism Aspergillus nidulans, the biotechnologically important fungus Aspergillus oryzae and the opportunistic human pathogen Aspergillus fumigatus was performed with focus on the general control/cross-pathway control ( $\mathrm{gc} / \mathrm{cpc}$ ) of amino acid biosynthesis. The extracted data were compared to other lower and higher eukaryotes as the bakers yeast Saccharomyces cerevisiae. Central part of the investigation is the gc/cpc but our results also encompass neighbouring pathways like the sensing of amino acids, their transport into the cells and regulation of protein biosynthesis from transcription to degradation of proteins. We found that the general control system is present in the investigated Aspergilli, but neighbouring systems partly differ from well characterized $S$. cerevisiae systems mainly in respect of amino acid sensing and transport. We also found that basic compounds of the translation machinery in Aspergilli rather resemble those of higher eukaryotes than of yeast. 


\subsection{Introduction}

The Aspergilli comprise a diverge group of filamentous fungi (Galagan et al., 2005). Among the over 185 Aspergilli are several species with impact on human health, including 20 human pathogens. In addition several economically, medically and agriculturally important fungal species are part of the Aspergillus family (Galagan et al., 2005).

Bioactive molecules like $\beta$-lactam antibiotics and aflatoxins are secreted by A. fumigatus and A. flavus (Brakhage et al., 2004; Gugnani, 2003; Sales and Yoshizawa, 2005). Additionally, A. fumigatus is an important human pathogen causing invasive aspergillosis in immunocompromised patients (Gagnadoux, 2005). A. oryzae is of high importance in asian countries, where it is used to produce sake, miso and soy sauce in industrial standards (Thammarongtham et al., 2001). A. nidulans constitutes a representative of this fungal genus that is capable of diverse and complex biosynthesis and differentiation processes like the well characterized sexual differentiation process where after mating with a compatible partner or "selfing" cleistothecia are formed which contain octades of ascospores (Brüggeman et al., 2003; Hoffmann et al., 2001a). During the last century the eukaryotic model organism was found to be easily investigated and manipulated by molecular methods. This makes Aspergillus species particularly suited for in depth studies on regulatory networks and cross-connections between environmental stimuli, metabolism and development and has steadily advanced our understanding of eukaryote physiology (Figure 11).

Amino acids are the building blocks for protein synthesis. The preferred nitrogen sources of Aspergilli are ammonium, glutamine or glutamate. Under energy limiting conditions amino acids can also serve as nitrogen and carbon sources in metabolism. All nitrogen related processes pass at some stage through the amino acids glutamine and glutamate where ammonium as nitrogen donor is easily transferred between these two amino acids and other keto-group containing molecules (Davis et al., 2005; Minehart and Magasanik, 1992). Fungi are able to use a variety of nitrogen sources like proteins, amino acids, nitrate, purines and even acetamide and specific permeases for different more or less preferred nitrogen sources (Davis et al., 2005; Marzluf, 1997). The biosynthesis of these permeases and enzymes needed for the related catabolism often have to be induced according to the availability in the environment. Nitrogen supply is carefully regulated in fungi and specific GATA transcription factors like AreA, Gln3p in yeast or NIT-2 in N. crassa regulate the synthesis of catabolic enzymes related to nitrogen (Davis et al., 2005; Marzluf, 1997). 
Amino acids are as well essential precursors for ribosomal biosynthesis of proteins as for nonribosomal products of secondary metabolism like $\beta$-lactam antibiotics synthesis (Brakhage, 1998; Brakhage et al., 2005). Most fungi prefer to take up amino acids from their diet since the synthesis includes several energy consuming steps. Amino acid uptake depends on the nutritional conditions in their surroundings and relies on sensors and uptake systems for these precursors. Most fungi are also able to secrete proteases into the medium to break down proteins in their medium and take up the remaining amino acids, though these actions are carefully regulated and mainly happen under stress conditions like nitrogen starvation and the presence of extra-cellular protein (Kredics et al., 2005; Ogrydziak, 1993; Pavlukova et al., 1998).

When no amino acids are available in the environment, these essential building blocks have to be either taken up from nutrient sources or de novo synthesized, whereas fungi, plants and prokaryotes are able to synthesize their amino acids, the uptake of amino acids from their diet is essential for mammals which are unable to produce their own amino acids. Fungal cells have to adjust rapidly to changing environmental conditions and therefore need to be able to build up or degrade proteins or other macromolecules and secondary metabolites in their surroundings. 


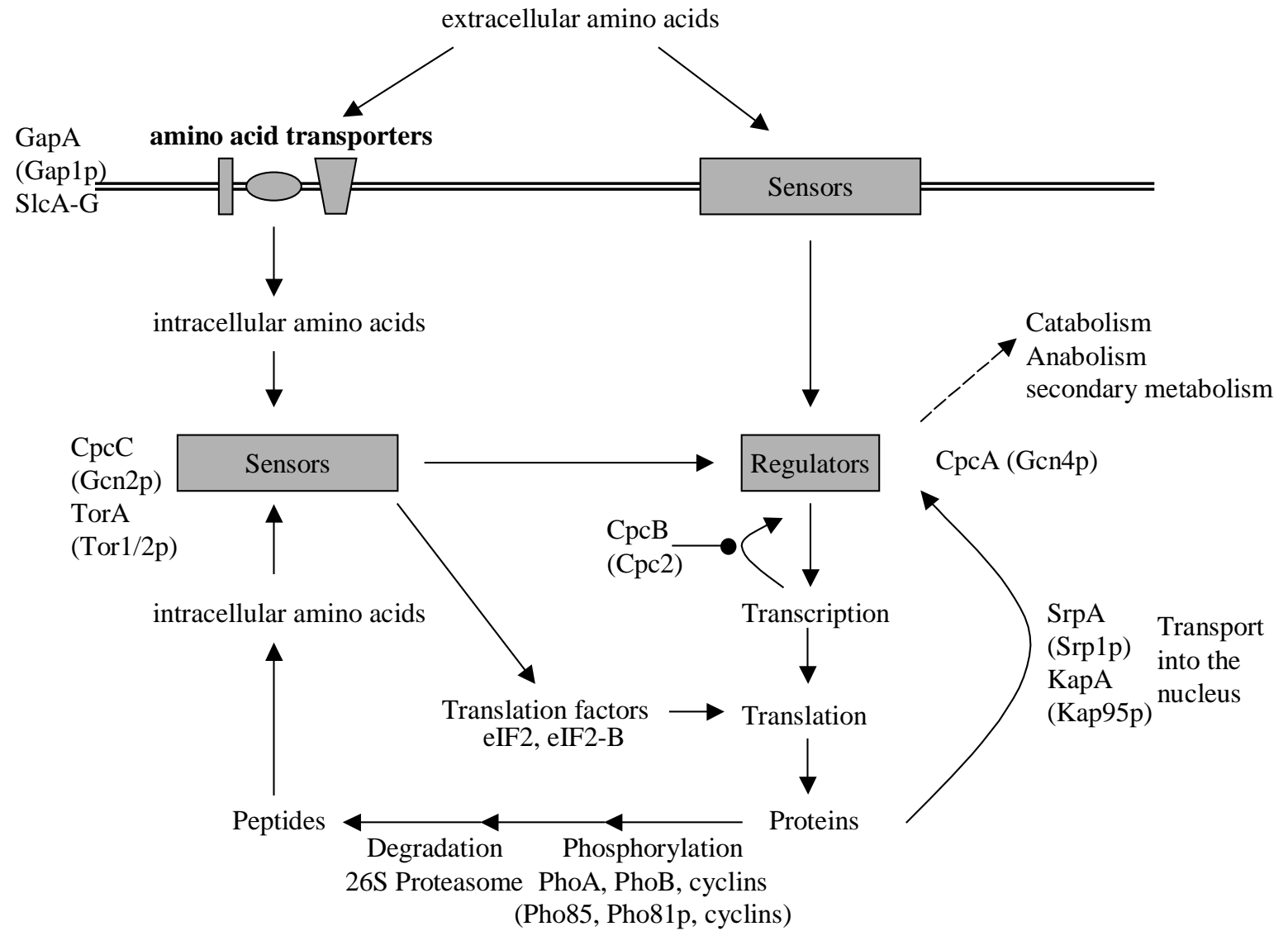

Fig. 11: Overview of the general control/cross-pathway control (gc/cpc) and neighbouring pathways of Aspergilli and $S$. cerevisiae

This figure shows a roadmap of cellular processes around the cross-pathway control and indicates related proteins for the respective processes. Extracellular amino acids are sensed by sensors in the cell wall and transported into the cells by a variety of transporters. Inside the cell amino acids are sensed by different sensors and signals are passed on to key regulators of the gc/cpc. According to amino acid availability the main transcription regulator enhances or decreases transcription of metabolic genes, translation can be increased or decreased. In times of nutritional hardship un-needed proteins can be degraded for their compounds or the central transcription factor can be degraded under non-limiting conditions in a regulatory manner (Klionsky, 2005). Under non-starvation conditions factors negatively regulate transcription of transcription factors (Schneper et al., 2004). 
Under very limiting conditions or if proteins are no longer required proteins can be broken down and their building blocks recycled to put to further use (Omura et al., 2001). Not only single proteins can be broken down in serious starvation or stress conditions, though. It is possible for the cell to do bulk degradations of large compartments of cells by autophagy in the vacuole (Klionsky, 2005). A controlled timely expression and destruction of proteins is a major regulatory mechanism in cellular processes (Hilt, 2004; Wittenberg and Reed, 2005). The main eukaryotic degradation machinery, the $26 \mathrm{~S}$ proteasome, specifically degrades ubiquitinylated proteins (Miller and Gordon, 2005). Proteolysis is performed by the ATPindependent peptidase activity of the $20 \mathrm{~S}$ core of the proteasome, whereas substrate specificity is mediated by the $19 \mathrm{~S}$ regulatory particle consisting of a base complex which confers ATPase and chaperon activity and a $26 \mathrm{~S}$ lid complex of so far unknown function (Takeuchi and Tamura, 2004). Proteins are targeted for degradation by poly-conjugation of the small ubiquitin protein. The first covalent binding is engaged between glycin 76 of ubiquitin and an epsilon-amino group of an internal lysin of the target protein and further isopeptidic elongation of the ubiquitin chain through lysine 48 of the first ubiquitin (Krappmann and Scheidereit, 2005; van Nocker and Vierstra, 1993). Newer insights to ubiquitin conjugation indicate that the alternative formation of ubiquitin chains through the lysine 63 residue do not mark a protein for degradation. This type of linkage, as opposed to the more typical Lys-48-linked chains, serves as a non-proteolytic marker of protein targets involved in error-free post-replicative DNA repair and NF-kappa B signal transduction (Krappmann and Scheidereit, 2005; McKenna et al., 2003). The corresponding ubiquitinylation cascade has been studied in-depth (Hershko and Ciechanover, 1998; Jackson and Eldridge, 2002). An ubiquitin activating enzyme (E1) activates the ubiquitin by a thioester linkage prior to transfer to a lysine residue of a specific protein substrate by a ubiquitin conjugating enzyme (E2). An ubiquitin ligase (E3) catalyzes substrate recognition and assists in ubiquitinylation. Several E3 ubiquitin ligase complexes are known, most prominent is the cullin-RING-H2-family that includes SCF (Skp1p/Cdc53p/F-box) (Deshaies, 1999; He et al., 2005; Tyers and Jorgensen, 2000) which plays an important role in the regulated destruction of the central transcription factor of the general control of amino acid biosynthesis Gcn4p of S. cerevisiae (Irniger and Braus, 2003).

The uptake of amino acids has to be carefully controlled and the reason for uptake has to be distinguished from another. One reason might be the uptake of amino acids as poor carbon or nitrogen sources in times of nitrogen or carbon starvation (Davis et al., 2005; Magasanik and Kaiser, 2002; Valenzuela et al., 2001), another the usual uptake of amino acids as building 
blocks for amino acid biosynthesis from their diet (Andreasson et al., 2004). If a fungus senses only poor nitrogen sources like proline in the diet, the nitrogen discrimination pathway or nitrogen catabolite repression (NDP or NCP) is required for their utilisation. GATA transcription factors like Gln3p, AreA or NIT-2 are activated to increase the production of enzymes needed for uptake and utilization (Abdel-Sater et al., 2004; Davis et al., 2005; Mo and Marzluf, 2003). The NDP is repressed if rich nitrogen sources are present (Boczko et al., 2005; Sosa et al., 2003). The first step needs to be in any case the sensing of amino acids outside of the cell (Forsberg et al., 2001). A subsequent signal cascade is needed to pass the signal for availability on into the cell and channel the signal into appropriate actions - to take up the amino acids as nitrogen or carbon sources or for anabolism and pass the signal on so that no longer required proteins can be degraded and recycled (Forsberg et al., 2001).

Most knowledge on the coordination network of amino acid uptake and metabolism was aquired in the bakers yeast Saccharomyces cerevisiae (Braus, 1991; Hinnebusch, 1986), but a lot is known in filamentous fungi like A. nidulans or N. crassa, as well (Busch et al., 2003; Ebbole et al., 1991; Eckert et al., 2000; Hoffmann et al., 2001b; Luo et al., 1995; Paluh and Yanofsky, 1991). A prominent example of such regulatory networks is the cross-pathway control of amino acid biosynthesis (cpc) or the general control of amino acid biosynthesis (gc). Originally the general control defined the increase of amino acid biosynthesis enzymes upon starvation on one amino acid (Carsiotis and Jones, 1974; Carsiotis et al., 1974), later followed by the discovery of global activators like Gcn2p and Gcn $4 p$ and repressors of the general control (Hinnebusch, 1985). This well studied complex regulatory network ensures increased transcription of a variety of amino acid biosynthetic genes when supply of a single amino acid is limited. This system is common in several filamentous fungi like Neurospora crassa, A. nidulans, A. fumigatus and A. niger (Hoffmann et al., 2001b; Krappmann et al., 2004; Paluh et al., 1988; Wanke et al., 1997). It is also found in the budding yeast Saccharomyces cerevisiae (Hinnebusch, 1984). The central transcription factor Gcn4p of $S$. cerevisiae is activated under amino acid limiting conditions. A transcription factor of a general control of amino acid biosynthesis itself can not be found in mammals, though c-jun, a human oncoprotein, shares high homologies to Gcn4p and CpcA. Activation of the general control is regulated via a complex regulatory cascade. The Gcn2p sensor kinase is attached to the ribosome in the cytoplasm. It monitors whether tRNAs are charged with amino acids. The protein controls the Gcn $4 p$ synthesis, but also controls the overall translation rate. Gcn2p-like kinases seem to be typical for eukaryotic cells. Interestingly there is a mammalian GCN2 which is induced by amino acid or serum starvation (Berlanga et al., 1999; Sood et al., 2000). 
If Gcn2p is replaced by its human counterparts, yeast Gen4p is synthesized independently of the presence or absence of amino acids (Dever et al., 1993). Gcn4p binds to cis-acting palindromic GCREs (general control response elements) 5'-ATGA (G/C) TCAT-3' in the promoter regions of amino acid biosynthetic target genes and thus enhances the transcription of these genes. For the filamentous fungi N. crassa and A. niger, homologues of this transcription factor have been described to bind on similar motifs called CPREs (crosspathway control response elements) (Hinnebusch, 1997; Hoffmann et al., 2001b). The homologue transcription factors in Aspergilli are known as CpcA or CPC-1 in N. crassa (Hoffmann et al., 2001b; Krappmann et al., 2004; Paluh et al., 1988). The regulation of the gc/cpc takes place on translational, transcriptional and protein stability level with various input signals and related pathways. The synthesis of these proteins is controlled in the cytoplasm. During translation the sensing of the on-site amino acid availability decides if the transcription factors are translated or not. The transcription factor needs to be transported into the nucleus after translation in the cytosol. In the nucleus the half-life of these transcription factors is regulated depending on amino acid availability, each of these checks ensuring a rapid response to many environmental stress conditions.

The general control seems to be not fully conserved in higher eukaryotes due to the inability of mammals to de novo synthesize all required amino acids. Nonetheless an amino acid sensing system similar to that of lower eukaryotes was found recently in the mammal piriform cortex, a region of the brain located in the rhinencephalon and part of the telencephalon related to olfaction. Certain nutrients, among others amino acids, possibly act through sensing mechanisms and have the capability to initiate cell-signalling events and regulate gene expression in the absence of hormonal influence (Hyde et al., 2003). Studies have shown that elevated amino acid levels sustain anabolism and inhibit catabolism in eukaryotic cells (Meijer and Dubbelhuis, 2004), meaning an increase in global mRNA levels and protein synthesis (Averous et al., 2003). Overall the intracellular amino acid limitation sensing and resulting processes in mammals seems similar to those in yeast, if a little more complex (Harding et al., 2000). It was shown that a lack of indespensable amino acids in mice leads to mGCN2 dependent phosphorylation of eIF2 $\alpha$ in mouse brains to restore indespensable amino acid homeostasis (Hao et al., 2005). The sensor kinase mGCN2 senses uncharged tRNAs and phosphorylates eIF2 $\alpha$ (Harding et al., 2000). The general downregulation of translation leads to expression of ATF-4 due to an upstream open reading frame in the mRNA similar to the mechanism known from yeast Gcn4p (Averous et al., 2003; Mielnicki et al., 1996). To induce expression of target genes, like CHOP (CCAAT/enhancer binding protein (C/EBP) 
homologous protein) (Bruhat et al., 1997) or asparagine synthethase AS (Hutson and Kilberg, 1994; Hutson et al., 1997) and the cationic amino acid transporter (Cat-1) (Fernandez et al., 2003) gene, ATF-2 needs to be phosphorylated as well (Averous et al., 2004).

Different mechanisms have been proposed where amino acid transporters may impact on mammalian signal transduction. The first one would be that an amino acid transporter acts as a substrate specific receptor and passes on a signal during transport of its substrate (Hyde et al., 2003). A second impact amino acid transporters could have is that during import of the amino acid substrate other molecules are symported or antiported impacting on intracellular changes of the cell physiology ( $\mathrm{pH}$, change in membrane potential, cellular volume change) (Hyde et al., 2003). A third impact amino acid transporters could have is the import of substrates and sensing through intracellular sensors like mGNC2 (Averous et al., 2003). Mammalian cells have a broad range of mechanisms for the transmembrane transport of amino acids (Hyde et al., 2003). The transporters have been classified into distinct "systems" defining substrate specificity, transport mechanism and regulatory properties (Hyde et al., 2003). The system can be divided up into layers. The first layer discriminates between sodium dependent and independent transporters. The second layer defines the charge of the substrate: neutral, anionic or cationic. The third layer defines the actual "system", which groups the transporters due to their substrate specificity (Hyde et al., 2003).

System BETA transporters belong to the SLC6 family and are mainly localized in the neuronal gaps. They transport the neurostransmitter amino acid GABA, but are also able to transport glycine and glutamate. The flux of GABA transporters is regulated by binding of diverse proteins to their N-terminus (Chen et al., 2004). SLC7 family transporters transport cationic, neutral and both amino acids (H, M, L, I, V, F, Y, W, Q) (Hyde et al., 2003; Wipf et $a l ., 2002)$. Along with the Ssylp protein of the yeast SPS system they belong to the family of APC (amino acid polyamine-choline) transporters (Verrey et al., 1999). Many of the transporters of the SLC7 family require the presence of glycoproteins to form a functional holotransporter in mammalian cells (Padbury et al., 2004). One of its members of this family, the LAT1 transporter, is suggested to act as an environmental sensor of amino acid availability (Padbury et al., 2004; Verrey et al., 1999). The SLC1 family of amino acid transporters transports glutamic acid and aspartic acid in astrocytes (Hyde et al., 2003). Astrocytes grow like filamentous fungi star shaped and form the brain-blood barrier in the brain (Volterra and Meldolesi, 2005). They also support the exchange of nutrients from blood to the nervous system. The glial glutamic acid transporter EAAT1 not only regulates glial signal transduction but is also important for development of the astrocytes as was shown for 
diseases like lissencephaly (Furuta et al., 2005; Zagami et al., 2005). The imino or SLC36 family of mammalian amino acid transporters is essential for uptake of degradation products from the lysosome and uptake of nutrients from the gut (Boll et al., 2004). The transporters were shown to effect or modulate growth through the TOR pathway in Drosophila in a yet not well characterized way (Goberdhan et al., 2005).

In this work we present the manual annotation and subsequent in depth genome analysis regarding the gc/cpc of the genomes of A. nidulans, A. oryzae and A. fumigatus. We analyzed the conservation of a general control-like system in these filamentous fungi, where not much more than the central transcription factor of the cross-pathway control was known (Hoffmann et al., 2001b; Krappmann et al., 2004; Wanke et al., 1997) and decided to broaden the analysis to the search for components both known in yeast, as well as in higher eukaryotes. The respective gene products were compared to those of Homo sapiens, Arabidopsis thaliana, Drosophila melanogaster, Neurospora crassa (in some cases to Magnaporthe grisea), Schizosaccharomyces pombe and Saccharomyces cerevisiae and gene names according to the rules of the genome project were assigned if necessary. 


\subsection{Methods}

\subsubsection{A. nidulans annotation and analysis}

The A. nidulans genome was annotated using the Calhoun annotation system (Galagan et al., 2002; Galagan et al., 2003; Galagan et al., 2005). The genome sequence was searched against the public protein databases using BLASTX (Altschul et al., 1997) with threshold $\mathrm{E} \leq 1 \mathrm{e}-5$. Genes were predicted using a combination of FGENESH, FGENESH+ (www.softberry.com) and GENEWISE (www.psc.edu/general/software/packages/genewise/genewise.html). Predicted genes were validated against ESTs aligned to the genome using BLAST (Altschul et al., 1997). All predicted proteins were searched against the PFAM set of hidden Markov models using the HMMER (http://hmmer.wustl.edu/) program and the public protein databases using BLASTP (Altschul et al., 1997).

Repeat sequences were detected by searching the genome sequence against itself using CrossMatch (http://www.genome.washington.edu/UWGC/analysistools/Swat.cfm), filtering for alignments longer than 200bp in length and clustering pairs based on region overlap.

$\begin{array}{llll}\text { Repeats were characterized } & \text { RepeatMasker }\end{array}$ (ftp.genome.washington.edu/RM/RepeatMasker.html) and RepBase (Jurka, 1998) followed by manual inspection. The A. fumigatus and A. oryzae genomes were annotated as described in separated reports (Nierman, W.C. et al., manuscript submitted, Machida, M. et al., manuscript submitted). For manual annotation the ERGO bioinformatics suite (Integrated Genomics, Chicago, USA) was used. 


\subsection{Results}

85 cross-pathway control related genes, or their proteins respectively, were investigated in all three Aspergilli (Table 1). In A. nidulans 77 homologues of these genes were found, 75 in $A$. oryzae and 72 in A. fumigatus indicating that processes may work differently in Aspergillus spec. than in the well studied general control of $S$. cerevisiae. The respective deduced protein sequences of $A$. nidulans were again blasted (tblastx) against a non-redundant database to confirm the given annotations and the returned hits against the above mentioned organisms were protocolled. 48 genes of the originally primarily annotated open reading frames were found for Homo sapiens, 44 genes for Arabidopsis thaliana, 49 genes for Drosophila melanogaster, 77 genes for Neurospora crassa, 64 genes for Schizosaccharomyces pombe and 69 genes for Saccharomyces cerevisiae. Twenty-eight of those genes were found to be the intersection and were used to generally compare the different organisms with A. nidulans on similarity level, showing an decreasing order of averaged homology from N. crassa to S. cerevisiae to $S$. pombe to A. thaliana to H. sapiens to D. melanogaster (Table 2).

\subsubsection{Sensing of extracellular and intracellular amino acids}

\subsubsection{The SPS amino acid sensing system}

The search for a system similar to the yeast SPS (Ssy1p - Ptr3p - Ssy5p) (Forsberg et al., 2001) amino acid sensor does not seem to exist in these three Aspergilli (nor can it be found in the genome of $N$. crassa). Ssylp is a protein consisting of 12 transmembrane spanning domains and strongly resembles an amino acid transporter but in contrast to these has an unusually elongated N-terminus which is required for activity. Similar transmembrane proteins can be found throughout the three Aspergillus genomes but none exhibits the elongated N-terminus indicating that a Ssylp homologue is not present. In addition a homologous region of the enlongated N-terminus can be found in neither of the three genomes. Neither Ptr3p nor Ssy5p could not be found in any of the Aspergilli (Forsberg et al., 2001). The SPS cleaves an NLS-masking domain from the heterodimeric transcription factor Stp1/2p when amino acids are present in the medium, sending it to the nucleus to enhance transcription of amino acid transporters (Andreasson and Ljungdahl, 2002). 


\subsubsection{Amino acid sensing through the TOR pathway}

The TOR pathway (in this specific context) is known to be another sensor of amino acid availability. Whereas in S. cerevisiae the two redundant TOR kinases Tor $1 p$ and Tor $2 p$ can be found, in each of the investigated Aspergilli only one gene for one protein similar to both TOR kinases of S. cerevisiae TorA could be found in the database with $48 \%$ similarity to the yeast TOR proteins, $58 \%$ similarity to $N$. crassa TOR, 54\% similarities to the TOR proteins of $S$. pombe and D. melanogaster, $44 \%$ similarity to human TOR and $42 \%$ similarity to the A. thaliana protein. The TOR kinase is known to interfere with gc/cpc in different ways. On the one hand inactivation of the TOR pathway in yeast results (among other processes) in a global translation repression and on the other hand during nitrogen starvation negatively affects the stability of high affinity amino acid transporters in the cell membrane (Cherkasova and Hinnebusch, 2003). If nitrogen is plentiful a kinase of the TOR-pathway phosphorylates Gen2p and thus inhibits the protein kinase domain and binding to tRNA of Gen2p (Cherkasova and Hinnebusch, 2003). Tap42p, a mediator of the TOR pathway can also be found in each of the Aspergilli. Tap42p is known to dephosphorylate Gen2p and induce Gcn4p transcription when the TOR kinase is inactive (Rohde et al., 2004; Wang et al., 2003). The Tap42p ortholog TapA can be found in all three Aspergilli, remarkably the similarity to the orthologs of higher eukaryotes lies under $30 \%$ whereas the fungal orthologs display similarities of $43,5 \%$ for $N$. crassa, 37\% for $S$. pombe and $34 \%$ for $S$. cerevisiae. 


\subsubsection{The gc/cpc sensor kinase of Aspergilli}

During starvation on amino acids the amount of uncharged tRNAs increases, which in yeast is sensed by the sensor kinase $\mathrm{Gcn} 2 \mathrm{p} / \mathrm{CpcC}$ and results in $\mathrm{CpcC}$ phosphorylating eIF-2 $\alpha$ thus lowering overall translation (Figure 12). The sensor kinase $\mathrm{CpcC}$ is present in the investigated Aspergillus species and the protein sequence is highly similar in all organisms (Zhu et al., 1996). The above effect is Gen1p/Gcn20p dependent (Vazquez de Aldana et al., 1995), both of which can be found in all three genomes. Their orthologs were named CpcD for Gcn1p and $\mathrm{CpcE}$ for Gcn20p. Interestingly no protein similar to $\mathrm{CpcE}$ can be found in A. thaliana, whereas the orthologs of $\mathrm{CpcD}$ are almost identical and so are the orthologs of the $\mathrm{CpcE}$ protein in the other compared organisms.

\subsubsection{Uptake of amino acids in the filamentous fungus A. nidulans}

\subsubsection{Amino acid uptake systems of Aspergillus species}

A prominent example of well characterized amino acid transporters in yeast can be found in all three Aspergillus species. Gap1p (general amino acid permease) of $S$. cerevisiae is a general high capacity amino acid permease which is known to transport all naturally occuring L-amino acids and also various D-amino acids (Jorgensen et al., 1998). Equally well known are the Trp/Tyr transporters Tat1p and Tat2p (Schmidt et al., 1994). All three Aspergillus species display an amino acid transport system GapA which is homologous to both the Gap1p and Tat $1 / 2 \mathrm{p}$ and shows similarities over $50 \%$ to its fungal counterparts (Table 3). If these transporters found in the Aspergilli are compared to higher eukaryotes no equivalents can be found. A homologue of the proline-specific permease Put4p called PutD can also be found in the Aspergilli with similarities of approximately $40 \%$ to the yeasts and Neurospora, but can not be found in higher eukaryotes. 

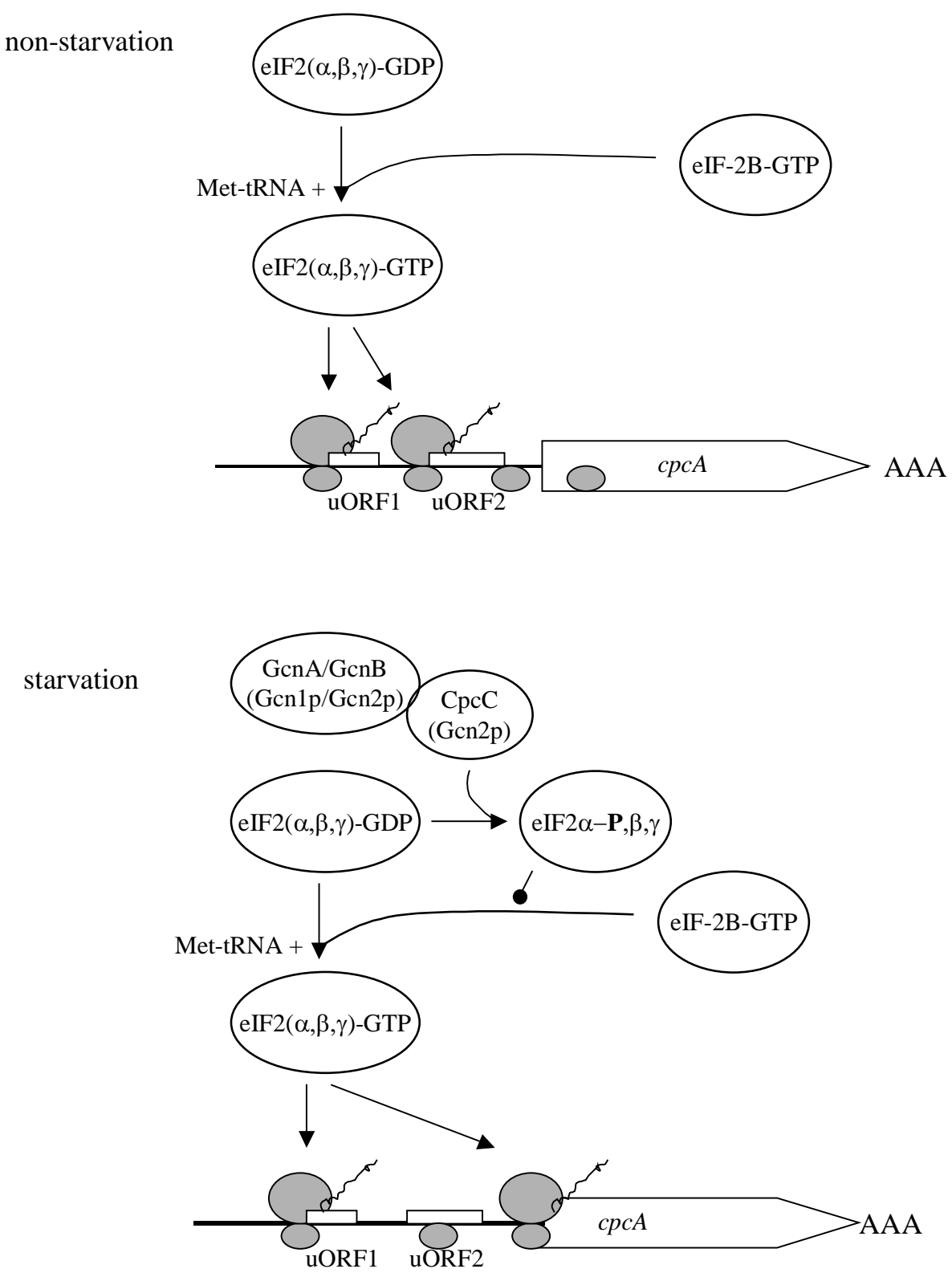

Fig. 12: Translational control of the gc/cpc regulator mRNA CpcA

Amino acids are either available or fungal cells starve for amino acids. Under non-starvation conditions $\mathrm{CpcC}$ is not active. When cells endure amino acid limitation, uncharged tRNA molecules are sensed by $\mathrm{CpcC}$ in cooperation with $\mathrm{GcnA} / \mathrm{GcnB}$. $\mathrm{CpcC}$ then phosphorylates the $\alpha$ subunit of the eukaryotic initiation factor eIF2. Phosphorylated eIF2a inhibits the eIF-2B complex, which acts as a guanine nucleotide exchange factor for eIF2. Inhibition of eIF-2B results in a global downregulation of translation due to lower amounts of charged tRNAs and GTP. The low amount of ternary complexes under starvation conditions leads to delayed reinitiation of the reassembled ribosome. This in turn leads to increased translation of the CpcA ORF instead of the uORFs under non-starvation conditions. The respective $S$. cerevisiae homologues to the Aspergillus proteins are shown in brackets (Hinnebusch et al., 2004). 


\subsubsection{Aspergillus amino acid uptake systems in comparison to mammalian counterparts}

In mammalian systems nutrients, especially amino acids, play an important role in the regulation of endocrine processes since mammalian systems are unable to de novo synthesize essential amino acids. In mammalian cells an SPS-like system is not present and it is not entirely clear how external signals are processed to the nucleus to react. Since there is no SPS system the sensing of amino acids in Aspergillus, sensing needs to work in a manner different from yeast. It might be possible that sensing of amino acids and propagation of the resulting signals takes place in the same manner as in cells of higher eukaryotes. It was shown that amino acid signals have a major impact on growth and development next to sensing the nutritional status and regulating transport of amino acids into the cell. This implies that amino acid transport might be similar to that in cells of higher eukaryotes, as well.

The Aspergillus genomes were searched for different mammalian amino acid transporter systems. We were able to find Aspergillus proteins that resemble different transporter families. In general similar proteins for each characterized amino acid transporter SlcA-F could be found in the genome of $N$. crassa or M. grisea (see table 1) with identities over $60 \%$.

Blast searches in the genomes carried out with members of the human SLC7 or L* family revealed proteins in all three Aspergillus genomes we named SlcA. They display a 30\% similarity to the human LAT1 transporter and $22 \%$ identity to a so far uncharacterized amino acid transporter of Drosophila. Similarities to any yeast protein are below 20\%. LAT1 is supposed to be an ubiquitously expressed transporter acting as an environmental amino acid sensor (Padbury et al., 2004; Verrey et al., 1999). It is characterized to transport mainly large hydrophobic amino acids like H, M, L, I, V, F, Y, W and Q (Hyde et al., 2003).

The SlcB protein was only found in A. nidulans. No gene coding for a similar protein was found throughout the genomes of the other Aspergilli, nor could any similar proteins be found in the investigated yeasts and the identity to Drosophila proteins was below 20\%. The SlcB protein shows $33 \%$ identity to the human EAA1 protein of the human system $\mathrm{X}_{\mathrm{AG}}^{-}$(SLC1 family) (Hyde et al., 2003). Members of the SLC1 family $\mathrm{K}+$ antiport glutamic acid and aspartic acid into the cells (Hyde et al., 2003). They are present in mammalian astrocytes (Gonzalez-Gonzalez et al., 2005). EAA1 plays an important role in astrocyte development, as was shown for diseases like lissencephaly (Furuta et al., 2005). 
The search for proteins similar to mammalian system imino transporters (SLC36) three proteins for each Aspergillus were found named SlcC, SlcE and SlcF, with relatively low homologies to the mammalian imino system transporters. SlcC is $30 \%$ similar to the so far uncharacterized hypothetical transporter FLJ39822, but shows $45 \%$ identity to yeast Avt2p (Russnak et al., 2001). SlcE and SlcF each show approximately 33\% identities to human proteins of the imino group and 50\%/45\% identities to the yeast Avt3 proteins (Russnak et al., 2001). The yeast proteins Avt $2 / 3$ and system imino transporters are involved in the transport of small neutral amino acids (Q, N, I, L and Y) from the lysosome/vacuole after bulk degradation of proteins (Russnak et al., 2001). So far known members of the imino family were shown to be involved in growth regulation by influencing the TOR pathway in Drosophila (Goberdhan et al., 2005). These findings indicate at least a role for these three Aspergillus transporters in a similar function to the Avt $2 / 3 \mathrm{p}$ of yeast.

A search for System BETA transporter revealed homologues only in human (32\% identity), fly (29\% identity) and $N$. crassa. An A. fumigatus homologue of these systems could not be found. The proteins found were named SlcD. System BETA transporters of the SLC6 family are known to transport GABA, glycine and glutamate (Hyde et al., 2003; Wipf et al., 2002). In summary, we found six different amino acid transporters, which are all present in A. nidulans, whereas A. oryzae lacks SlcB and A. fumigatus SlcB and SlcD. Phylogenetic analyses of the three Aspergilli and Neurospora show that these fungi originate from a common ancestor and that A. nidulans and N. crassa separated first from the ancestor, the other two Aspergilli separated later from each other (Galagan et al., 2005). The transporters $\mathrm{SlcC} / \mathrm{E} / \mathrm{F}$ resemble transporters that are known to transport amino acids to or from the lysosome, which indicates the cellular function of these proteins (Boll et al., 2004; Russnak et al., 2001). SlcA is similar to the human LAT1 APC transporter, indicating a possible role in extracellular amino acid sensing (Padbury et al., 2004). SlcB is only present in A. nidulans and $N$. crassa indicate that the other Aspergilli lost the gene encoding SlcB after separation from A. nidulans. Regarding its relationship with EAA1 it might be possible that one function of this protein might be involvement in (polar)growth in A. nidulans besides amino acid uptake (Furuta et al., 2005; Zagami et al., 2005). The gene coding for SlcD is not present in A. fumigatus, which indicates a loss of the gene after separation from A. oryzae. 


\subsubsection{Regulation of fungal protein biosynthesis in response to intracellular amino acid availability}

\subsubsection{Regulation of expression of the central transcription factor of $\mathrm{gc} / \mathrm{cpc}$ in Aspergillus species}

Due to the special structure of the promoter of the transcription factor $\mathrm{CpcA}$ of the $\mathrm{gc} / \mathrm{cpc}$ of Aspergillus the translation of the $c p c A-m R N A$ is increased under starvation conditions resulting in more CpcA protein (Hoffmann et al., 2001b). CpcA is able to positively autoregulate its own transcription, as well as the transcription of amino acid biosynthesis genes through $\mathrm{CpcA}$ recognition elements (CPREs) under starvation conditions (Hoffmann et al., 2001b). The orthologs of CpcA are hard to find at a first glance. Similarities are mainly found in the C-terminal leucine zipper region of $\mathrm{CpcA}$ which is responsible for DNA-binding. The leucine zipper motif is conserved throughout all organisms from fungus to man. The leucine zipper can also be found in the human c-Jun and Fos-family of proteins, thus these proteins are counted to the c-Jun like family. The leucine zippers though consisting of 1 to 5 leucine residues are fully interchangeable and restore functionality in complementation experiments (Kouzarides and Ziff, 1989).

$\mathrm{CpcB}$ of $A$. nidulans is a $\mathrm{G} \beta$-like protein homologous to the mammalian RACK1 repressing the transcription of CpcA under non-starvation conditions (Hoffmann et al., 1999). The protein is constitutively expressed in A. nidulans (Hoffmann et al., 1999). The yeast homologue Cpc2 was shown to interact directly with the $40 \mathrm{~S}$ subunit of the ribosome providing a platform for other ribosome bound proteins during translation with their propeller-like WD40 repeats at the mRNA exit site of the ribosome (Nilsson et al., 2004; Sengupta et al., 2004; Shor et al., 2003). RACK1 and its homologues are found to be highly conserved in all organisms investigated.

Interestingly the third intron of $A$. nidulans $c p c B$ is conserved in the $S$. cerevisiae $C P C 2$ and $N$. crassa $c p c-2$ genes and harbors the U24 small nucleolar RNA (snoRNA) coding region (Hoffmann et al., 1999; Qu et al., 1995). The U24 snoRNA is required for site specific 2'-o-methylation of 25S rRNA (Nazar, 2004). Though the coding region can be found in the ascomycete A. nidulans its deuteromycete family members A. fumigatus and A. oryzae do not harbor the coding region of the U24 snoRNA anywhere near $\mathrm{CpcB}$. 


\subsubsection{The elongation initiation factor eIF-2 of Aspergillus}

For initiation of translation the eukaryontic ribosomal pre-initiation complex scans mature mRNA towards the 3 -end. On arrival at the AUG Start codon the 80s ribosomes finally assemble (Preiss and Hentze, 2003). The translation machinery needs phosphorylated eIF$2 \gamma$, which is subsequently dephosphorylated during translation and it needs to be recycled by the guanine nucleotide exchange factor GEF (eIF-2B) to maintain steady translation (Preiss and Hentze, 2003). By phosphorylation to further activate eIF-2 $\gamma$ the translation is repressed due to stoichiometrically decreasing GEF and a subsequent lack of the initiation factor (Clemens, 1994; Kapp and Lorsch, 2004; Preiss and Hentze, 2003). Each of the components of the initiation factor eIF-2 share high similarities to the components of the initiation factors of other organisms. Among the Aspergilli the components of the involved factors can be found easily, indicating a similar mechanism of translation control as in yeast. The alpha, beta and delta subunits of the GEF eIF-2B are well conserved between all compared organisms. The epsilon subunit is better conserved in the investigated fungi than in man, fly and plant, with similarities to its fungal counterparts of $>42 \%$ and to the other eukaryotes $<30 \%$. The gamma subunit of eIF-2B is not too well conserved at all, though orthologs can be found in all compared organisms, the highest homology is shared with $N$. crassa followed by H. sapiens. The eukaryotic translation initiation factor eIF3 functions by interacting with eIF2 and stabilizing the interaction between the ternary complex (composed of eIF2.GTP.Met-tRNA $A_{i}$ ) and the $40 \mathrm{~S}$ ribosomal subunit, thereby forming the $43 \mathrm{~S}$ ribosomal complex (Chaudhuri et al., 1999). Twelve well conserved subunits of eIF3, eIF-C(a-1) can be found in the three Aspergilli, whereas only six are present in the yeast $S$. cerevisiae and only nine in S. pombe. Nearly no conservation at all can be found for subunit eIF3j to the higher eukaryotes, but it is found highly conserved to its counterpart of $N$. crassa. The eIF3, the lid of the proteasome and the Cop9 signalosome are regulatory multiprotein complexes whose components can be characterized through the specific PCI (proteasome, Cop9, eIF3) or MPN (Mpr1p, Pad1 Nterminal) protein domains (see table 1, see chapter 2)(Glickman et al., 1998; Kapelari et al., 2000; Kim et al., 2001). 


\subsubsection{Transport of the transcription factor into the nucleus}

Nuclear import is essential for Gcn4p to induce transcription of numerous genes whose products are involved in many different biosynthetic pathways in $S$. cerevisiae during response to amino acid starvation, glucose starvation and other stresses (Engelberg et al., 1994; Hinnebusch and Natarajan, 2002; Mösch et al., 1990; Natarajan et al., 2001). CpcA as counterpart of Gen $4 \mathrm{p}$ in A. nidulans needs to be transported to the nucleus, as well, to play its role as a transcription factor during amino acid starvation response (Pries et al., 2002). It was shown by Pries et al., 2004 that the $\alpha$-importin Srp1p and the $\beta$-importin Kap95p act as a heterodimer to channel Gcn $4 p$ into the yeast nucleus via the nuclear pores. So far no experimental procedures have been carried out to find whether similar proteins take over this task in A. nidulans or any other fungal species. Blast searches in the now available Aspergillus and N. crassa genomes revealed that proteins with high identities to Srp1p (over 58\%) and Kap95p (over 39\%) can be found to be encoded in the available Aspergillus and

Neurospora crassa genomes. The assigned ORF for the Srp1p homologue SrpA was annotated as AN2142.1 and the homologue to the Kap95p homologue KapA was annotated as AN0906.1 during the automated and manual annotation of the A. nidulans genome. Blast searches in the available Aspergillus nidulans genome revealed that there is only one $\alpha$-importin like protein and twelve members of the importin beta superfamily present (see chapter 4), which means that the Aspergillus genomes harbor one less beta importin member than yeast.

\subsubsection{Regulation of the protein level of the central transcription factor of the gc/cpc}

The yeast transcription factor Gcn $4 p$ is a highly unstable protein with a half-life of apr. 5 minutes under non-starvation conditions. A well conserved PEST region (Kornitzer et al., 1994) and ubiquitinylation sites are responsible for the instability of this protein and its subsequent ubiquination by the SCF complex and degradation in the 26s proteasome (Irniger and Braus, 2003; Meimoun et al., 2000). Nothing is known about the half-life of its fungal counterparts so far. The cyclin dependent kinases Pho85p and Srb10p are responsible for destabilisation of the transcription factor (Irniger and Braus, 2003; Qiu et al., 2004). The homologues PhoA and SrbA were found to be highly conserved in all investigated organisms, still a homologue of SrbA can not be found in A. thaliana. PhoA shows $81 \%$ identity to the 
N. crassa protein and $72 \%$ and $67 \%$ to the proteins of S. pombe and S. cerevisiae respectively. The identity compared to the proteins of higher eukaryotes is $>55 \%$. SrbA was found to be $51 \%$ identical to $N$. crassa and $45 \%$ and $49 \%$ identical to the respective proteins of $S$. pombe and S. cerevisiae. The respective proteins of Drosophila and human showed $36 \%$ and $42 \%$ identity respectively. We could find a corresponding cyclin Srb11p, named SrbB in the Aspergilli, but like in all investigated cyclins the identity to other proteins was generally low $(<40 \%)$ only the proteins of $N$. crassa and S. cerevisiae showed identities of $47 \%$ and $42 \%$ respectively. Under non-starvation conditions Pcl5p is required for Pho85p mediated Gcn4p degradation in yeast (Shemer et al., 2002). The cyclin dependent kinase Pho85p was shown to phosphorylate Gcn4p at T165 and thus mark it for ubiquitination and further degradation at the 26s proteasome (Meimoun et al., 2000). Ten different cyclins are known to interact with the cyclin dependent kinase Pho85p. A search throughout the genome of the Aspergilli revealed relatively low homologies for a cyclin further called PclA with identities of 53\% and $35 \%$ to yeast the proteins Pcl1p and Pcl2p respectively. PclL displayed $46 \%$ identity to Pho80p and $71 \%$ identities to Nuc-1 of N. crassa. PclE displayed a low identity of only $34 \%$ to Pcl5p and even lower identities of $34 \%$ and $28 \%$ to the proteins of Arabidopsis and S. pombe. The identity to the Neurospora protein is $45 \%$. PclF resembles Pcl6p and Pcl7p with higher identity to Pcl6p (38\%) and high identity to the Neurospora protein (60\%). PclH shows less than $20 \%$ identity to Pcl10p, but $28 \%$ identity to Pcl8p. The Neurospora protein is $60 \%$ identical to PclH. PclI is $49 \%$ identical to Pcl9p and the respective Neurospora protein, whereas PclK shows only $26 \%$ identity to $\mathrm{Clg} 1 \mathrm{p}$ and $36 \%$ identity to its other fungal counterparts. Generally it has to be remarked that the cyclins are relatively weakly conserved among the investigated organisms. Comparing the Aspergillus CpcAs and Gcn4p of S. cerevisiae one finds the phosphorylation site T165 well conserved through the Aspergilli. It is notable that the phosphorylation site is exchanged from threonine to serine in the Aspergilli and that the site is not conserved in N. crassa (Figure 13). 

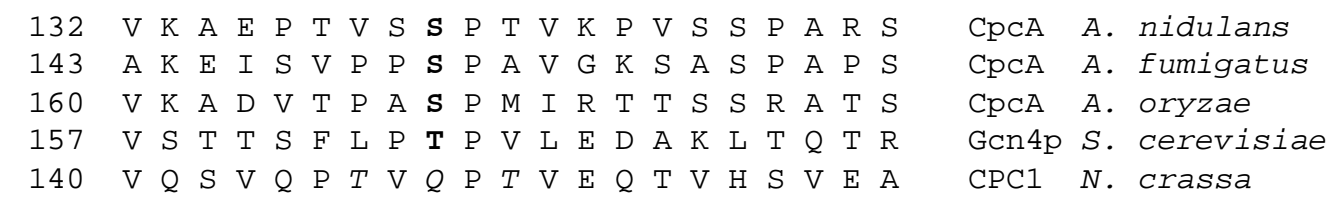

Fig. 13: The degradation-related phosphorylation site of CpcA-like proteins is highly conserved In yeast it was shown that phosphorylation of Gen4p at Thr165 leads to rapid degradation of the protein (Meimoun et al., 2000). Multialignments of the phosphorylation site Thr165 of S. cerevisiae with their homologues of Aspergillus species and Neurospora crassa show that the phosphorylation site is highly conserved from yeast to Aspergillus, though instead of threonine the amino acid serine is used as a phosphorylation site in Aspergilli. The respective amino acids are depicted in bold. In N. crassa no conservation of this site can not be found, though neighbouring threonines (italic) might play a role in phosphorylation and further degradation of CPC1.

Phosphorylated Gcn4p is subsequently ubiquitinylated by the $\mathrm{SCF}^{\mathrm{Cdc} 4}$ ligase which targets it to degradation by the $26 \mathrm{~S}$ proteasome, whereas the SCF complex is constitutive and Gen $4 p$ stability is subject to the phosphorylation state of Gen4p mediated by Pho85p (Kornitzer et al., 1994; Meimoun et al., 2000). The E3 ubiquitin ligase specific for Gcn4p consists of the cullin Cdc53p, Skp1p, binding the specific F-box protein, the RING-H2 protein Hrt1p and the specificity protein Cdc4p (Irniger and Braus, 2003)(Figure 14). We were able to identify homologues of these proteins in all three Aspergilli under the names CulA, SkpA (also known as SconC (Piotrowska et al., 2000)), HrtA and CdcD respectively. SkpA or SconC was earlier characterized as a Skp1p-like protein interacting with the F-box protein SconB as negative regulators of the sulphur-metabolism in A. nidulans (Piotrowska et al., 2000). The cullin and the specificity protein $\mathrm{CdcD}$ are highly conserved among all organisms with overall identities of $>56 \%$ for the cullin and $>40 \%$ identities among compared F-box proteins. We were unable to find a homologue of $\mathrm{CdcD}$ in plant. The Aspergillus genomes also revealed Rub1p homologues with identities $>50 \%$ to the proteins of the other compared organisms (S. Busch, personal communication). 


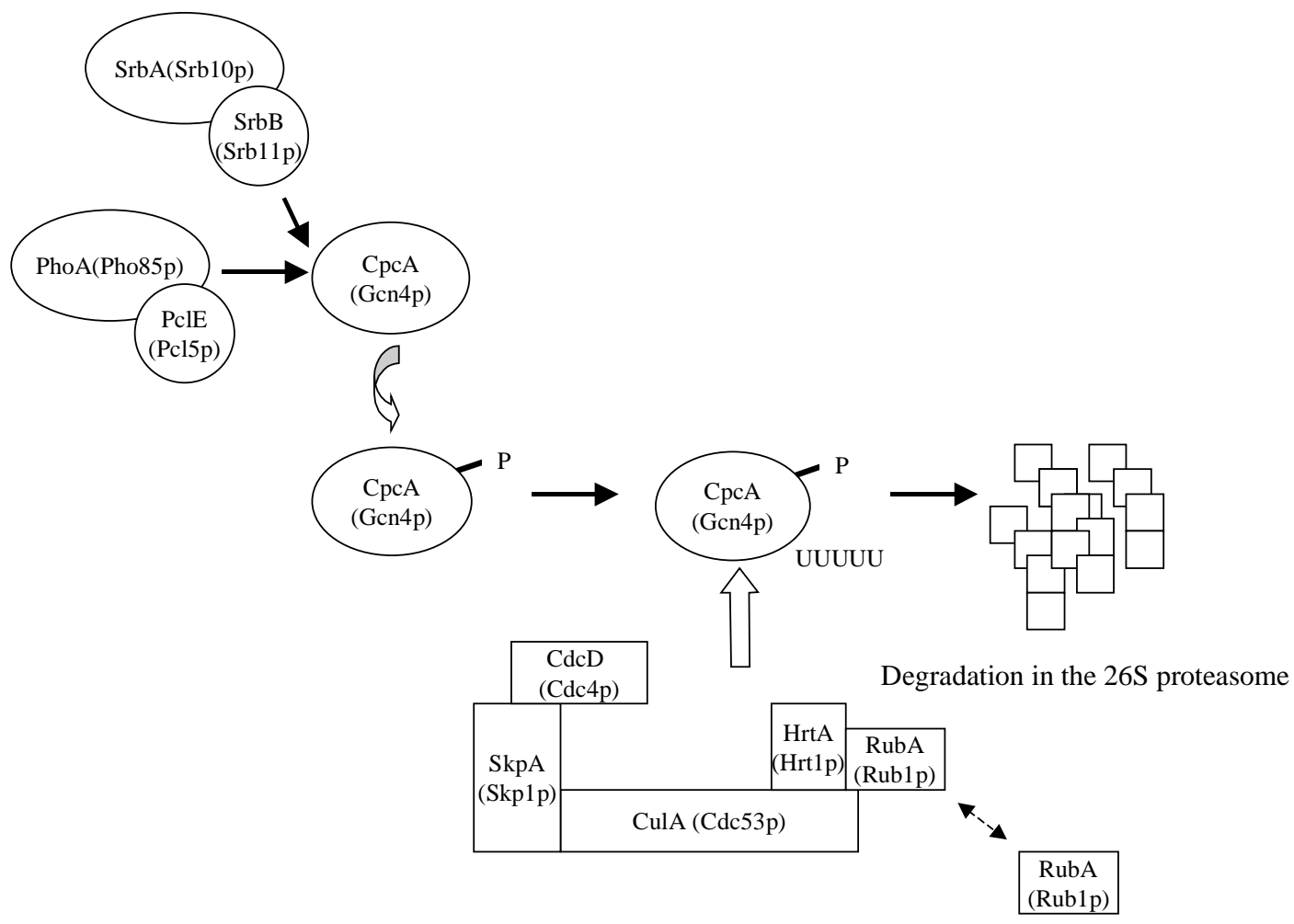

Fig. 14: Degradation of the transcription factor CpcA

Degradation of $\mathrm{CpcA}$ is initiated by phosphorylation of specific threonine residues. The $\mathrm{CpcA}$ degradation is triggered by two cyclin dependent kinases SrbA and PhoA. The second step of the degradation is the polyubiquitinylation of $\mathrm{CpcA}$ mediated by the $\mathrm{E} 3$ ubiquitin ligase complex $\mathrm{SCF}^{\mathrm{CdcD}}$, targeting the protein for degradation at the $26 \mathrm{~S}$ proteasome. The $\mathrm{SCF}^{\mathrm{CdcD}}$ activity is regulated by dynamic neddylation/deneddylation (see chapter 2).

\subsubsection{Fungal amino acid biosynthesis investigated on selected examples}

Since all three Aspergilli are able to grow without amino acid supplementation they should be able to synthesize all amino acids. We had a closer look at five biosynthetic pathways - the biosynthesis of histidine and lysine accompanied by the putative production of the branching secondary metabolism - the penicillin biosynthesis - and the formation of tryptophan, tyrosine and phenylalanine. For the ease of reading the respective names of the $S$. cerevisiae proteins have been added in brackets to their respective Aspergillus homologues. 


\subsubsection{Histidine biosynthesis}

Histidine is produced by six biosynthetic enzymes (EC numbers are added to the attached table, suggested names were also added) HisA (His1p), HisB (His3p), HisC (His4p), HisD (His2p), HisE (His5p) and HisHF (His7p) starting from PRPP (Figure 15). HisA is an ATP phosphoribosyltransferase forming phosphoribosyl-ATP. The next two steps are accomplished by HisC (trifunctional histidinol dehydrogenase) forming first phosphoribosylAMP, then phosphoribosylformimino AICAR-P, the branchpoint to the purine metabolism. HisHF forms imidazolglycerole-3-phosphate (Künzler et al., 1993; Valerius et al., 2001). The next step is taken over by HisB forming imidazoleacetol-phosphate (Busch et al., 2001). HisE then forms L-histidinol-phosphate which is converted to L-histidinol by HisD. The last two steps are accomplished by HisC forming first L-histidinal, then L-histidine. The genes for histidine biosynthesis were found to be highly conserved amongst the three Aspergillus species.

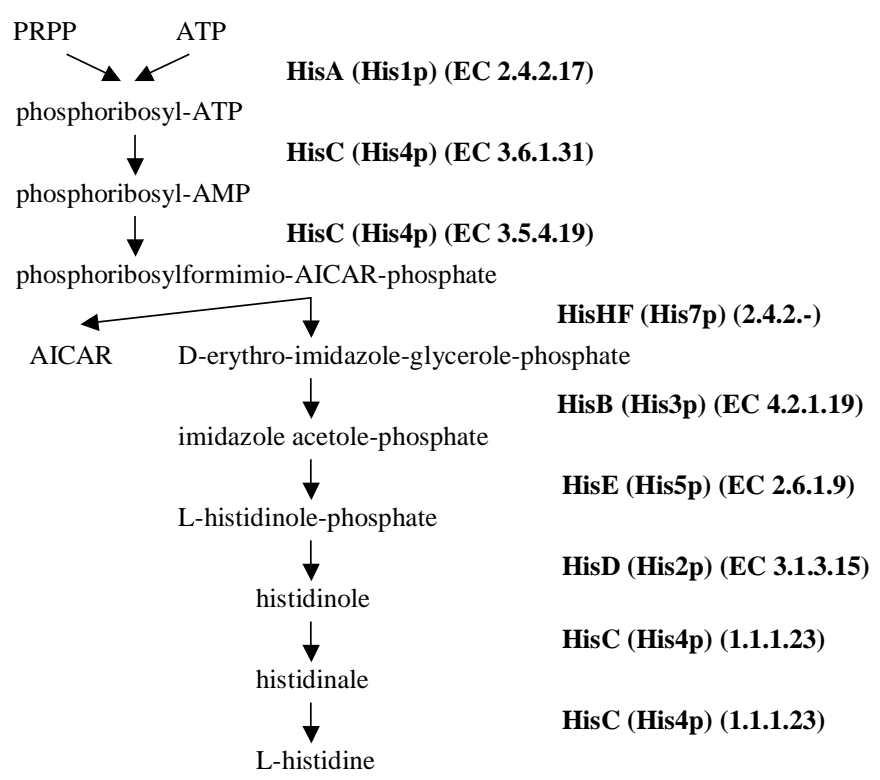

Fig. 15: Histidine biosynthesis and associated enzymes

The catalytic steps of the histidine biosynthesis are described. The enzymes catalysing the enzymatic reactions are depicted in bold. The respective $S$. cerevisiae homomlogues to the Aspergillus proteins are shown in brackets. 


\subsubsection{Lysine and penicillin biosynthesis of Aspergilli}

The amino acid lysine is produced from aspartate through the diaminopimelate (DAP) pathway in most bacteria and higher plants. In fungi, in the thermophilic bacterium Thermus thermophilus, and in several archaeal species, lysine is synthesized by a completely different pathway called the $\alpha$-aminoadipate pathway (Nishida et al., 1999). In A. nidulans the penicillin biosynthesis pathway branches from the lysine pathway. It was suggested that upon amino acid starvation the cross-pathway control overrules penicillin biosynthesis and favors lysine production (Busch et al., 2003) (Figure 16).

Lysine biosynthesis starts with homocitrate which is converted by LysF (Lys4p) to HomoIsocitrate, which is then turned over by LysE (Lys12p) to 2-Oxoadipate. LysD then forms L2-Aminoadipate from 2-Oxoadiapate. L-2-Aminoadipate-6-semialdehyde is then formed by LysB (Lys2p) from L-2-Aminoadipate. The Saccharopine dehydrogenase LysG (Lys9p) then forms L-Saccharopine, which is then made to L-Lysine by LysA (Lys1p). All necessary enzymes can be expressed in all three Aspergilli. One can observe that the proteins of the penicillin metabolism as a secondary metabolism starting from L-2-Aminoadipate are only present in A. nidulans and A. oryzae. Penicillin is synthesized in a three step reaction starting from L-2-Aminoadipate mediated by the enzymes AcvA, IpnA and AatA, leading to the assumption that $A$. oryzae is able to produce penicillin, as well as A. nidulans, whereas A. fumigatus lacks the necessary enzymes.

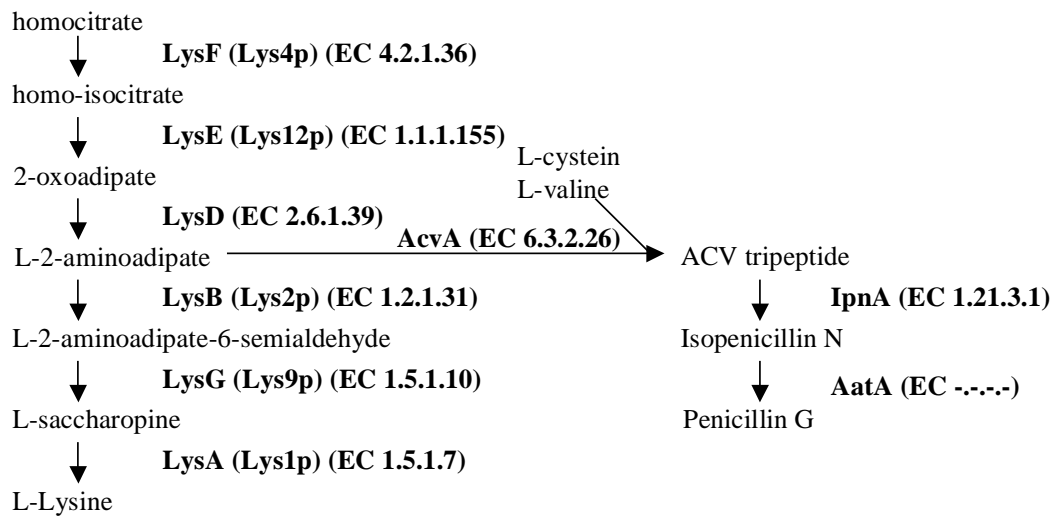

Fig. 16.: The $\alpha$-aminoadipate pathway of lysine biosynthesis and penicillin biosynthesis branch of A. nidulans and $A$. oryzae

The catalytic steps of the lysine and penicillin biosynthesis are described. The enzymes catalyzing the enzymatic reactions are depicted in bold. At the $\alpha$-aminoadipate step the cells are able to choose between two biosynthetic ways. Penicillin synthesis could either be reduced to favor lysine biosynthesis or increased to kill food sources and use them as amino acid or nitrogen sources. The respective $S$. cerevisiae homologues to the Aspergillus proteins are shown in brackets. 


\subsubsection{Aromatic amino acid biosynthesis}

The biosynthetic cascade resulting in the aromatic amino acids L-phenylalanine, L-tyrosine and L-tryptophan links carbohydrate metabolism to biosynthesis of aromatic compounds. Whereas animals are only able to form tyrosine by hydroxylation of phenylalanine and therefore require this amino acid together with tryptophan in their diet, bacteria, plants and fungi are competent to synthesize all three aromatic amino acids de novo (Sirtori et al., 2005). The shikimate pathway leads to the formation of chorismate, the last common intermediate of the three pathways (Quevillon-Cheruel et al., 2004). After that the three pathways divide into the tryptophan biosynthetic branch and the biosynthesis of prephenate, which then divides up into the biosynthetic branch of phenylalanine and tyrosine production (Figure 17).

\subsection{Tryptophan biosynthesis}

The intermediate chorismate is transformed in a two step reaction to anthranilate by the anthranilate synthase $\operatorname{TrpA}(\operatorname{Trp} 2 \mathrm{p})$ and the trifunctional glutamine amidotransferase/ $\mathrm{N}-\left(5^{\prime}-\right.$ phosphoribosyl)anthranilate isomerase/indole-3-glycerol transferase (Hütter et al., 1986). TrpD (Trp4p), the phosphoribosyl transferase then forms N-(5-phospho-b-D-ribosyl)anthranilate, which is then subsequently transformed to 1-(2-carboxyphenylamino)-1-deoxyD-ribulose-5-phosphate and (3-indolyl)-glycerol phosphate by $\operatorname{TrpC}(\operatorname{Trp} 3 \mathrm{p})$ (Yelton et al., 1983). The final step, the formation of tryptophan, is taken over by $\operatorname{TrpB}(\operatorname{Trp} 5 \mathrm{p})$ the phosphoribosyl transferase (Hütter and DeMoss, 1967).

\subsection{Tyrosine and phenylalanine biosynthesis}

After the formation of prephenate from chorismate by AroC (Aro7p) (Andrews et al., 1973), the chorismate mutase, the phenylalanine/tyrosine specific branch of the aromatic amino acid biosynthesis branches in two alternative routes to form the end products (Krappmann et al., 1999). One proceeds via the formation of phenylpyrovate by PhaA (Pha1p), a dehydratase followed by transamination by AroH (Aro8/9p) to phenylalanine (Jensen and Fischer, 1987). On the other hand 4-hydroxyphenylpyrovate is formed by TyrA (Tyr1p), which is subsequently transaminated by AroH to tyrosine (Jensen and Fischer, 1987). 


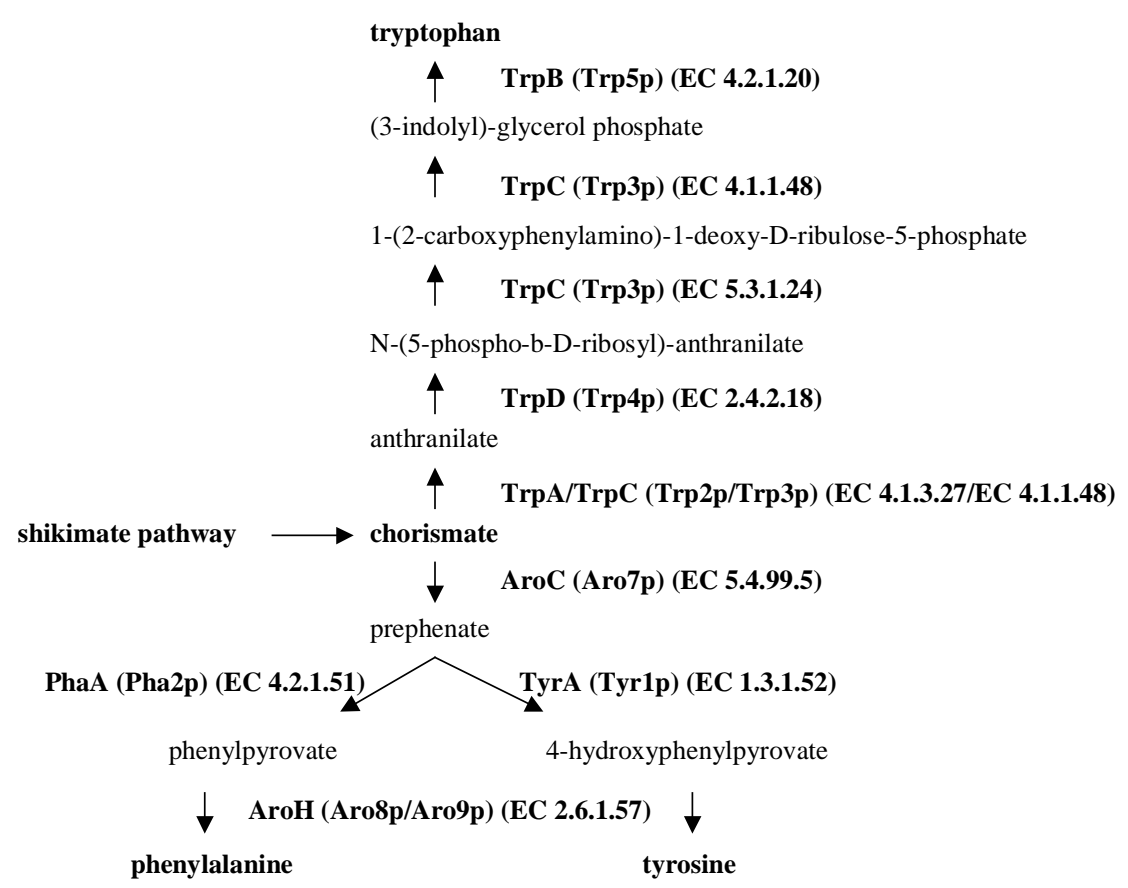

Fig. 17.: The aromatic amino acid biosynthesis of Aspergillus species

The catalytic steps of the tryptophan, phenylalanine and tyrosine biosynthesis are described. The enzymes catalyzing the enzymatic reactions are depicted in bold. The shikimate pathway leads to the last common intermediate of the aromatic amino acid biosynthesis, chorismate, where the pathways divide into the tryptophan branch and the tyrosine/phenylalanine branch. The respective $S$. cerevisiae homologues to the Aspergillus proteins are shown in brackets. 


\subsection{Discussion}

The A. nidulans genome sequence and our comparative analysis with the genome sequences of A. fumigatus and A. oryzae was shown in comparison to higher eukaryotes and other fungi. The genomic annotation in context with general control and cross-pathway control opens new perspectives and broadens our field of view on this field of research. A complex overview of the $\mathrm{gc} / \mathrm{cpc}$ related genes and proteins is given in this comparative paper. The regulation of transcription, translation and post-translational regulation of the central transcription factor $\mathrm{CpcA}$ and in addition the uptake and sensing of amino acids were being addressed.

We were able to in silico identify the central components of a gc/cpc that are yet only known from $S$. cerevisiae. On the other hand we found that other systems accompanying the gc/cpc partly differ from yeast, mainly on behalf of the transport and sensing of extracellular amino acids. Interestingly we found that the elements of a basic system like the translation machinery much more resemble their orthologs of higher eukaryotes than the compared yeasts. It was shown by Hoffman et al., 2001 that CpcA is capable of autoregulating its own transcription under amino acid starvation conditions. Under non-starvation conditions this autoregulatory effect is inhibited by $\mathrm{CpcB}$ in a yet unclear mechanism (Hoffmann et al., 2000). It could be shown, that necessary genes and their respective proteins needed for translational regulation of the expression of $\mathrm{CpcA}$ are available in all three Aspergilli. We were able to identify the sensor kinase $\mathrm{CpcC}$ which is presumably able to sense the availability of intracellular amino acids and phosphorylate eIF-2 $\alpha$, a part of the translation machinery, under amino acid starvation conditions which in turn represses translation in general, though the translation of $\mathrm{CpcA}$ increases. The known parts of the elongation initiation factor 2 known to be involved in gc/cpc were identified in the Aspergilli. Generally we found the genes for the subunits of the elongation initiation factor well conserved towards the other compared fungi, whereas the guanine nucleotide exchange factor eIF-2B $\varepsilon$ subunit is far more similar to the orthologs of higher eukaryotes. Interestingly the epsilon subunit is not only the largest but also the catalytic subunit of the complex (Gomez et al., 2002). It was shown that mutations in the gene for eIF- $2 \mathrm{~B} \varepsilon$ can exhibit a decrease in complex formation following decreased GTP/GDP exchange rate resulting altered mRNA transcription and lead to leukoencephalopathy, the vanishing of white matter (VWM), which is a severe inherited human neurodegenerative disorder in man (Li et al., 2004). Due to the high similarity of the respective proteins in Aspergillus, an easily genetically manipulated organism, genetic and 
biochemical research on the effect of mutations in the respective genes may help understand the manifestation of this wasting desease.

All twelve subunits of the elongation factor eIF3 were found to be present in Aspergillus, in contrast to $S$. cerevisiae where only 6 subunits can be found and to $S$. pombe, where only nine of the subunits can be found. The subunits eIF3j and eIF3k were only very weakly conserved to those of higher eukaryotes and one can only speculate if the genome harbors genes encoding for these proteins due to the lack of physical evidence.

According to our data the transport into the nucleus of the central transcription factor of the gc/cpc might be similar to the mechanisms taking place in yeast, at least the necessary factors are present in the Aspergilli. Nothing is so far known about the half-life of the protein in the nucleus or in the cytosol. The presence of putative proteins involved in yeast in Gcn $4 p$ targeting and degradation in the $26 \mathrm{~S}$ proteasome in Aspergillus indicates similar mechanisms for CpcA degradation. An additional hint is the conservation of the phosphorylatable Thr165 residue, though in all investigated Aspergilli this residue was found to be exchanged for a serine residue. Interestingly an alignment of this protein region shows that this phosphorylatable residue is not conserved in $N$. crassa, though phosphorylatable threonine residues can be found in the direct vicinity of the expected spot (Figure 13).

Several mechanisms have been described and proposed for different amino acid uptake systems in mammalian cells. These amino acid uptake systems seem in general not only regulatable, some of them seem to transmit signals of amino acid abundance to directly or indirectly regulate corresponding cellular responses. These mechanisms are so far not well understood. We were able to identify at least four proteins $\mathrm{SlcB}$ and $\mathrm{SlcC} / \mathrm{E} / \mathrm{F}$ in silico that may have a similar effect on development and growth as their heigher eukaryotic relatives. SlcB resembles transporters of the SLC1 family. The function of EAA1, a member of the SLC1 family, was recently shown to have a direct effect on the morphology of astrocysts, star-like glial cells. Dysregulation of this glutamate transporter expression leads to disorganized cortex formation and altered astrocytic phenotypes, as was shown for type II lissencephaly patients and cell lines (Furuta et al., 2005; Zagami et al., 2005). On the other hand do imino amino acid transporters directly or indirectly influence cellular growth in fly through the TOR pathway (Goberdhan et al., 2005). Aspergillus has so far proven to be a good model for amino acid dependent growth and regulation, since a dysregulation of intracellular amino acid biosynthesis leads to an arrest in fruitbody formation (Busch et al., 2001; Eckert et al., 1999; Hoffmann et al., 2000; Valerius et al., 2001). In this context it would be interesting to find new mechanisms reacting to amino acid starvation conditions that 
have an influence on development and growth. The mechanisms of amnio acid regulated growth and development regulation are of great therapeutic interest since there are a lot of pathological circumstances associated with dysregulation of amino acid metabolism (anthropomorphic lateral sclerosis, altered amino acid availability/transport in tumor cells and tissue response to insulin). Nutritional or pharmaceutical intervention through such mechanisms would be of great benefit. Thus the findings of amino acid transporters similar to those of mammals in filamentous fungi (but not in other lower eukaryotes) may open the way for another field of research for these model organisms.

As examples of amino acid biosyntheses we were able to prove the existence of the necessary genes encoding for the proteins for histidine, lysine, tyrosine, tryptophan and phenylalanine biosynthesis, demonstrating that the investigated Aspergilli are able to produce these amino acids if no extracellular sources are available. In contrast, mammals are unable to produce all amino acids and have to rely on taking essential amino acids (isoleucine, leucine, lysine, methionine, phenylalanine, threonine, tryptophane, valine) and semi-essential amino acids, that can substitute for essential amino acids under certain conditions (arginine, histidine, tyrosine, cysteine), up from their diet. In secondary metabolism A. nidulans is known to produce penicillin utilizing an intermediate of the lysine biosynthesis. Strikingly the genes encoding for the proteins conducting the enzymatic reactions to produce penicillin could be found in A. oryzae, but not in A. fumigatus rendering it unable to produce penicillin.

Concluding, we find that the basic regulatory cascade of regulation of amino acid biosynthesis is very similar to that of higher eukaryotes, if a little bit less complex regarding the activation of target genes. External (SPS-system) or internal (GCN2, TOR) sensors sense amino acid abundance and react to depletion by lowering the overall translation rate through eIF2. This leads to increased expression of transcription factors like Gcn4p, CpcA or CHOP and increased transcription of target genes. The transcription factor of the $\mathrm{gc} / \mathrm{cpc}$, the regulation of cellular expression and probably regulation of its stability seem rather to resemble those of yeast and other fungi, than higher eukaryotes. The sensing and uptake system of amino acids at least in part is more complex than in yeast. Amino acid transporters were found that are not present in yeast, but in higher eukaryotes like human and a yeast-like SPS amino acid sensing system is not present. This might indicate ways of uptake and sensing that are similar to those of higher eukaryotes and might have an impact on development and growth. 
Table 1: Homology of A. fumigatus and A. oryzae to A. nidulans genes. Similarities with e-values $<$ E-50 are shaded green, e-values $<$ E-20 are shaded orange and e-values $>$ E-20 are shaded yellow

\begin{tabular}{|c|c|c|c|c|c|c|c|c|}
\hline & Protein & Coding sequence & H. sapiens & A. thaliana & $\begin{array}{c}D . \\
\text { melanogaster } \\
\end{array}$ & N. crassa & S. pombe & S. cerevisiae \\
\hline$a n$ & \multirow[t]{3}{*}{ CpcA } & AN3675.1 & & & & \multirow{3}{*}{$\begin{array}{c}2,00 \mathrm{E}-09 \\
\text { CPC-1 }\end{array}$} & & \multirow{3}{*}{$\begin{array}{c}3,00 \mathrm{E}-07 \\
\text { Gcn } 4 p\end{array}$} \\
\hline$a f$ & & 58.m08917 & & & & & & \\
\hline$a o$ & & 20032.m00013 & & & & & & \\
\hline & \multirow{4}{*}{$\mathrm{CpcC}$} & AN2246.1 & & \multirow{4}{*}{$\begin{array}{c}2,00 \mathrm{E}-58 \\
\text { Gcn2p } \\
\text { homologue }\end{array}$} & \multirow{4}{*}{$\begin{array}{c}\text { 2,00E-76 } \\
\text { eIF2a kinase }\end{array}$} & \multirow{4}{*}{$\begin{array}{c}0 \\
\text { Gen2p }\end{array}$} & \multirow{4}{*}{$\begin{array}{c}1,00 \mathrm{E}-154 \\
\text { CPC-3 }\end{array}$} & \multirow{4}{*}{$\begin{array}{c}0 \\
\text { Gen2p }\end{array}$} \\
\hline an & & AN2246.1 & \multirow{3}{*}{$\begin{array}{c}0 \\
\text { eIF2a kinase }\end{array}$} & & & & & \\
\hline$a f$ & & 71.m15880 & & & & & & \\
\hline ao & & 20163.m00313 & & & & & & \\
\hline$a n$ & \multirow{3}{*}{$\mathrm{CpcB}$} & AN4163.1 & \multirow{3}{*}{$\begin{array}{c}1,00 \mathrm{E}-139 \\
\text { GNB2L1 }\end{array}$} & \multirow{3}{*}{$\begin{array}{c}1,00 \mathrm{E}-118 \\
\text { AAL34190.1 }\end{array}$} & \multirow{3}{*}{$\begin{array}{c}\text { 1,00E-129 } \\
\text { Rack1 }\end{array}$} & \multirow{3}{*}{$\begin{array}{c}1,00 \mathrm{E}-177 \\
\text { CPC-2 }\end{array}$} & \multirow{3}{*}{$\begin{array}{c}1,00 \mathrm{E}-136 \\
\text { Cpc2 }\end{array}$} & \\
\hline$a f$ & & $58 . \mathrm{m} 07362$ & & & & & & $\mathrm{Cpc} 2 \mathrm{p}$ \\
\hline ao & & 20179.m00653 & & & & & & \\
\hline & & & & & & & & \\
\hline$a n$ & eIF2b & AN2992.1 & $3,00 \mathrm{E}-50$ & $2,00 \mathrm{E}-46$ & $2,00 \mathrm{E}-47$ & 2,00E-91 & $5,00 \mathrm{E}-63$ & $3,00 \mathrm{E}-62$ \\
\hline$a f$ & (Sui3p) & 59.m09022 & EIF2beta & EIF2 beta & EIF2 beta & EIF2 beta & EIF2 beta & EIF2 beta \\
\hline ao & & 20174.m00396 & & & & & & Sui3p \\
\hline & & & & & & & & \\
\hline an & eIF $2 \alpha$ & AN3156.1 & 4,00E-71 & 1,00E-72 & 2,00E-65 & $4,00 \mathrm{E}-118$ & 3,00E-97 & $4,00 \mathrm{E}-95$ \\
\hline$a f$ & $(G \mathrm{cn} 3 \mathrm{p})$ & $59 . \mathrm{m} 08547$ & EIF2 alpha & EIF2 alpha & EIF2 alpha & EIF2 alpha & EIF2 alpha & EIF2 alpha \\
\hline ao & & 20093.m00085 & & & & & & Gcn3p \\
\hline & & & & & & & & \\
\hline$a n$ & eIF2 $\gamma$ & AN4470.1 & 0 & $4,00 \mathrm{E}-173$ & 0 & 0 & 0 & 0 \\
\hline$a f$ & (Gcd11p) & $58 . \mathrm{m} 07860$ & EIF2 gamma & EIF2 gamma & EIF2 gamma & EIF2 gamma & EIF2 gamma & EIF2 gamma \\
\hline$a o$ & & 20142.m00265 & & & & & & Gcd11p \\
\hline an & eIF2-B $\chi$ & AN0978.1 & $800 \mathrm{~F}-20$ & 0.001 & $500 \mathrm{~F} 08$ & $400 \mathrm{~F}-40$ & $200 F_{-10}$ & $500 \mathrm{~F}_{10}$ \\
\hline$a f$ & (Gcdlp) & 70.m15616 & EIF2B3 & , & AAG38016 & CAD21057 & Tif223 & Gcdlp \\
\hline ao & & 20178.m00545 & & & & & & \\
\hline & & & & & & & & \\
\hline$a n$ & eIF2-B $\delta$ & AN6864.1 & $5,00 \mathrm{E}-76$ & $1,00 \mathrm{E}-71$ & $5,00 \mathrm{E}-62$ & $2,00 \mathrm{E}-73$ & $1,00 \mathrm{E}-89$ & 1,00E-79 \\
\hline$a f$ & $(\mathrm{Gcd} 2 \mathrm{p})$ & $71 . \mathrm{m} 15275$ & EIF2B4 & AAC23414 & EAL25620 & CAE76139 & CAA91965 & Gcd2p \\
\hline$a o$ & & 20151.m00246 & & & & & & \\
\hline & & & & & & & & \\
\hline$a n$ & eIF2-B $\beta$ & $\begin{array}{l}\text { AN1344.1 } \\
\end{array}$ & $6,00 \mathrm{E}-53$ & $2,00 \mathrm{E}-58$ & $2,00 \mathrm{E}-44$ & 2,00E-66 & $1,00 \mathrm{E}-76$ & 1,00E-56 \\
\hline af & $($ Gcd7p) & $\begin{array}{l}70 . \mathrm{m} 14889 \\
\end{array}$ & EIF2B4 & AAF20216 & NP_570020 & CAD71011 & CAB52277 & Gcd7p \\
\hline ao & & 20084.m00045 & & & & & & \\
\hline & & & & & & & & \\
\hline$\frac{a n}{a f}$ & $\begin{array}{l}\text { elF2-B } \alpha \\
(\mathrm{Gcn} 3 \mathrm{p})\end{array}$ & $\begin{array}{c}\text { AN0167.1 } \\
71 . \mathrm{m} 15442 \mid\end{array}$ & $\begin{array}{c}\text { 1,00E-46 } \\
\text { EIF2B1 }\end{array}$ & $\begin{array}{r}1,00 \mathrm{E}-46 \\
\mathrm{AAF} 02861\end{array}$ & $\begin{array}{r}6,00 \mathrm{E}-42 \\
\mathrm{AAG} 38014\end{array}$ & $\begin{array}{r}5,00 \mathrm{E}-52 \\
\mathrm{XP} 323697\end{array}$ & $\begin{array}{r}2,00 \mathrm{E}-66 \\
\text { CAB57849 }\end{array}$ & $\begin{array}{c}2,00 \mathrm{E}-62 \\
\mathrm{G} \operatorname{cn} 3 \mathrm{n}\end{array}$ \\
\hline ao & & 20158.m00275 & & & & & & \\
\hline & & & & & & & & \\
\hline an & eIF2-B $\varepsilon$ & manual annotation & $2,00 \mathrm{E}-91$ & 2,00E-99 & $1,00 \mathrm{E}-60$ & $7,00 \mathrm{E}-123$ & $5,00 \mathrm{E}-129$ & $6,00 \mathrm{E}-101$ \\
\hline$a f$ & (Gcd6p) & $69 . \mathrm{m} 15713$ & eIF2-B epsilon & CAB78832 & AAG38017 & XP_331190 & Tif225 & Gcd6p \\
\hline$a o$ & & 20178.m00726 & & & & & & \\
\hline & & & & & & & & \\
\hline an & CpcE & AN4315.1 & $4,00 \mathrm{E}-165$ & & 2,00E-164 & 0 & 0 & 0 \\
\hline$a f$ & & 58.m08009 & ABCF3 & & NP_649129 & CAE85618 & NP_595837 & Gen20p \\
\hline ao & & 20067.m00019 & & & & & & \\
\hline & & & & & & & & \\
\hline an & CpcD & AN5840.1 & 0 & 0 & 0 & 0 & 0 & 0 \\
\hline$a f$ & & 72.m18976 & HsGCN1 & NP_176659 & EAA46127 & XP_325658 & CAA92385 & Gcnlp \\
\hline$a o$ & & 20177.m00380 & & & & & & \\
\hline & & & & & & & & \\
\hline$a n$ & elF3a & 10049.m00076 & e-97 & e-83 & e-78 & 0.0 & e-135 & e-65 \\
\hline$a f$ & (Tif32p) & 54.m06688 & NP_003741 & NP_192881 & NP_649470 & XP_322126 & NP_596379 & NP_009635 \\
\hline ao & & 20175.m00540 & eIF3a S10 & eIF3a & eIF3 S10 & & eIF3p110 & Tif32p \\
\hline & & & & & & & & \\
\hline an & elF3b & 10006.m00160 & e-130 & e-117 & e-112 & 0.0 & e-169 & e-121 \\
\hline$a f$ & (Prt1p) & 54.m06392 & NP_874371 & NP_568498 & NP_611228 & XP_330984 & NP_594528 & NP_015006 \\
\hline ao & & 20155.m00213 & eIF3b S9 & eIF3b & eIF3 S9 & & 3 beta & Prtlp \\
\hline
\end{tabular}




\begin{tabular}{|c|c|c|c|c|c|c|c|c|}
\hline & Protein & Coding sequence & H. sapiens & A. thaliana & $\begin{array}{c}D . \\
\text { melanogaster }\end{array}$ & N. crassa & S. pombe & S. cerevisiae \\
\hline an & \multirow[t]{3}{*}{ eIF3c (Nip1p) } & 10119.m00059 & \multirow{3}{*}{$\begin{array}{c}\text { e-75 } \\
\text { AAC27674 } \\
\text { eIF3c S8 } \\
\end{array}$} & \multirow{3}{*}{$\begin{array}{c}\mathrm{e}-72 \\
\text { AAC } 83464 \\
\text { eIF3c } \\
\end{array}$} & \multirow{3}{*}{$\begin{array}{c}\text { e-65 } \\
\text { NP_611242 } \\
\text { eIF3 S8 } \\
\end{array}$} & \multirow{3}{*}{$\begin{array}{c}\mathrm{e}-121 \\
\text { XP_328537 }\end{array}$} & \multirow{3}{*}{$\begin{array}{c}\text { e-143 } \\
\text { NP_593828 } \\
\text { eIF3 p98 } \\
\end{array}$} & \multirow{3}{*}{$\begin{array}{c}\text { e-104 } \\
\text { NP_014040 } \\
\text { Niplp } \\
\end{array}$} \\
\hline$a f$ & & $89 . \mathrm{m} 02023$ & & & & & & \\
\hline $\overrightarrow{a o}$ & & 20153.m00210 & & & & & & \\
\hline$a$ & \multirow{3}{*}{ eIF3d } & 10129.m00312 & \multirow{3}{*}{$\begin{array}{c}\text { e-93 } \\
\text { NP_003744 } \\
\text { elF3d S7 } \\
\end{array}$} & \multirow{3}{*}{$\begin{array}{c}\mathrm{e}-86 \\
\text { NP_193830 } \\
\text { eIF3d }\end{array}$} & \multirow{3}{*}{$\begin{array}{c}\text { e-91 } \\
\text { NP_524463 } \\
\text { eIF3 p66 }\end{array}$} & \multirow{3}{*}{$\begin{array}{c}0.0 \\
\text { XP_327666 }\end{array}$} & \multirow{3}{*}{$\begin{array}{c}\mathrm{e}-145 \\
\text { NP_594625 } \\
\text { moe1 }\end{array}$} & \\
\hline$\frac{a b}{a f}$ & & $72 . \mathrm{m} 19557$ & & & & & & \\
\hline ao & & 20114.m00102 & & & & & & \\
\hline & & & & & & & & \\
\hline$a n$ & elF3e & 10051.m00454 & e-106 & $\mathrm{e}-100$ & e-87 & e-162 & e-102 & \\
\hline$a f$ & & 59.m08758 & AAC51917 & NP_567047 & NP_477385 & XP_325744 & NP_595367 & \\
\hline$a 0$ & & 20175.m00410 & eIF3e S6 & eIF3e & Int6 & & eIF3 p48 & \\
\hline & & & & & & & & \\
\hline an & eIF3f & & e-38 & e-36 & e-30 & e-96 & e- 85 & \\
\hline$a f$ & & $70 . \mathrm{m} 14877$ & XP_290345 & NP_181528 & NP_649489 & XP_326514 & CAA16829 & \\
\hline$a 0$ & & 20074.m00037 & eIF3f S5 & eIF3f & & & (CSN6) & \\
\hline & & & & & & & & \\
\hline$a n$ & elF3g (Tif35p) & 10103.m00031 & e-36 & e-31 & e-30 & e-61 & $e-52$ & e-32 \\
\hline$a f$ & & 72.m19937 & NP_003746 & AAG53636 & NP_57001 & XP_328752 & 595727 & NP_010717 \\
\hline$a o$ & & 20177.m00635 & eIF3g S4 & eIF3g & & & eIF3 p33 & Tif35p \\
\hline & & & & & & & & \\
\hline$a n$ & elF3h & 10017.m00197 & e-42 & e-51 & e-29 & e-109 & e-54 & \\
\hline$a f$ & & 70.m14937 & NP_003747 & NP_563880 & NP_524834 & XP_328635 & NP_593158 & \\
\hline ao & & 20169.m00238 & eIF3h S3 & eIF3h p38 & & & & \\
\hline & & & & & & & & \\
\hline$a n$ & eIF3i (Tif34p) & 10051.m00544 & $e-99$ & $e-73$ & $\mathrm{e}-84$ & $e-143$ & $\mathrm{e}-120$ & e-104 \\
\hline$a f$ & & 59.m09019 & NP_003748 & NP_182152 & NP_523478 & XP_323195 & NP_594958 & NP_013866 \\
\hline ao & & 20174.m00390 & eIF3i S2 & eIF3i & TRIP1 & & eIF3 p39 & Tif34p \\
\hline & & & & & & & & \\
\hline$a n$ & eIF3j (Hcrlp) & 10098.m00332 & 0.0005 & e-0,6 & 0.36 & e-29 & & $\mathrm{e}-07$ \\
\hline$a f$ & & $69 . \mathrm{m} 15631$ & NP_003749 & NP_850918 & NP_610541 & XP_328660 & & NP_013293 \\
\hline ao & & 20178.m00416 & eIF3j $\quad \mathrm{S} 1$ & eIF3j & eIF3j & & & $\bar{H} c r 1 p$ \\
\hline & & & & & & & & \\
\hline$a n$ & eIF3k & 10051.m00602 & & e-15 & & & & \\
\hline$a f$ & & 59.m08960 & NP_037366 & NP_195051 & NP_611604 & XP_330357 & & \\
\hline$a o$ & & 20174.m00289 & eIF3k & eIF3k & & & & \\
\hline & & & & & & & & \\
\hline$a n$ & eIF31 & 10101.m00131 & e-115 & e-79 & e-92 & & & \\
\hline$a f$ & & 72.m19170 & NP_057175 & NP_680222 & NP_648553 & XP_326134 & & \\
\hline$a o$ & & 20177.m00684 & eIF31 & IF31 & & & & \\
\hline & & & & & & & & \\
\hline$a n$ & 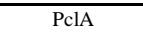 & AN0453.1 & & & & $2,00 \mathrm{E}-48$ & & $3,00 \mathrm{E}-31$ \\
\hline$a f$ & & 54.m06646 & & & & XM_324203 & & Pcllp \\
\hline$a o$ & & manual annotation & & & & & & \\
\hline & & & & & & & & \\
\hline$a n$ & 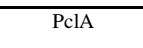 & AN0453.1 & & & & $2,00 \mathrm{E}-48$ & & $3,00 \mathrm{E}-31$ \\
\hline$a f$ & & 54.m06646 & & & & XM_324203 & & Pcl2p \\
\hline ao & & manual annotation & & & & & & \\
\hline & & & & & & & & \\
\hline an & PclL & AN5156.1 & & $1,00 \mathrm{E}-13$ & & $5,00 \mathrm{E}-50$ & $6,00 \mathrm{E}-11$ & $1,00 \mathrm{E}-26$ \\
\hline$a f$ & & 54.m06862 & & NM_130038 & & XM_328176 & AL021747 & Pho80p \\
\hline$a o$ & & 20128.m00091 & & & & Nuc-1 & & \\
\hline & & & & & & & & \\
\hline$a n$ & PclE & AN9500.1 & & $6,00 \mathrm{E}-05$ & & $6,00 \mathrm{E}-44$ & $9,00 \mathrm{E}-20$ & $1,00 \mathrm{E}-07$ \\
\hline$a f$ & & 62.m03402 & & NM_130072 & & XM_331476 & Z98975 & Pcl5p \\
\hline ao & & 20043.m00015 & & & & & & \\
\hline & & & & & & & & \\
\hline$a n$ & $\overline{\mathrm{PclF}}$ & AN3755.1 & & $3,00 \mathrm{E}-04$ & & $9,00 \mathrm{E}-63$ & $1,00 \mathrm{E}-13$ & $3,00 \mathrm{E}-26$ \\
\hline$a f$ & & 65.m07369 & & NM_130038 & & XM_331979 & AL021747 & Pcl6p \\
\hline ao & & 20146.m00204 & & & & & & \\
\hline & & & & & & & & \\
\hline$\overline{a n}$ & $\mathrm{PclF}$ & AN3755.1 & & $3,00 \mathrm{E}-04$ & & $9,00 \mathrm{E}-63$ & $1,00 \mathrm{E}-13$ & $3,00 \mathrm{E}-26$ \\
\hline$a f$ & & 65.m07369 & & NM_130038 & & XM_331979 & AL021747 & Pcl7p \\
\hline ao & & 20146.m00204 & & & & & & \\
\hline
\end{tabular}


Chapter 3

\begin{tabular}{|c|c|c|c|c|c|c|c|c|}
\hline & Protein & Coding sequence & H. sapiens & A. thaliana & $\begin{array}{c}D . \\
\text { melanogaster }\end{array}$ & N. crassa & S. pombe & S. cerevisiae \\
\hline$a n$ & \multirow[t]{3}{*}{$\mathrm{PclH}$} & AN5825.1 & & & \multirow{3}{*}{$\begin{array}{c}1,00 \mathrm{E}-15 \\
\text { NM_115919 }\end{array}$} & \multirow{3}{*}{$\begin{array}{c}3,00 \mathrm{E}-37 \\
\text { BX284753 }\end{array}$} & & \multirow{3}{*}{$\begin{array}{c}1,00 \mathrm{E}-15 \\
\text { Pclsp }\end{array}$} \\
\hline$a f$ & & 72.m18950 & & & & & & \\
\hline ao & & 20097.m00074 & & & & & & \\
\hline & & & & & & & & \\
\hline$a n$ & \multirow[t]{3}{*}{ PclI } & AN0453.1 & & & & \multirow{3}{*}{$\begin{array}{c}2,00 \mathrm{E}-48 \\
\text { XM_324203 }\end{array}$} & & \multirow{3}{*}{$\begin{array}{c}\text { 3,00E-31 } \\
\text { Pcl9p }\end{array}$} \\
\hline$a f$ & & 54.m06646 & & & & & & \\
\hline$a o$ & & manual annotation & & & & & & \\
\hline & & & & & & & & \\
\hline$a n$ & \multirow[t]{3}{*}{$\mathrm{PclH}$} & AN5825.1 & & & \multirow{3}{*}{$\begin{array}{c}1,00 \mathrm{E}-15 \\
\text { NM_115919 }\end{array}$} & \multirow{3}{*}{$\begin{array}{c}3,00 \mathrm{E}-37 \\
\text { BX284753 }\end{array}$} & & \multirow{3}{*}{$\begin{array}{l}\text { 1,00E-15 } \\
\text { Pcl10p }\end{array}$} \\
\hline af & & 72.m18950 & & & & & & \\
\hline$a o$ & & 20097.m00074 & & & & & & \\
\hline & & & & & & & & \\
\hline$a n$ & \multirow[t]{3}{*}{ PclK } & AN4984.1 & & & \multirow{3}{*}{$\begin{array}{c}\text { 6,00E-06 } \\
\text { BT015746 }\end{array}$} & \multirow{3}{*}{$\begin{array}{c}5,00 \mathrm{E}-40 \\
\text { XM_327578 }\end{array}$} & $2,00 \mathrm{E}-22$ & $2,00 \mathrm{E}-18$ \\
\hline$a f$ & & 59.m08886 & & & & & Z98270 & $\mathrm{Clg} 1 \mathrm{p}$ \\
\hline ao & & 20125.m00144 & & & & & & \\
\hline & & & & & & & & \\
\hline an & PhoA & AN8261.1 & $1,00 \mathrm{E}-97$ & $3,00 \mathrm{E}-93$ & $1,00 \mathrm{E}-97$ & $5,00 \mathrm{E}-143$ & $4,00 \mathrm{E}-115$ & $2,00 \mathrm{E}-109$ \\
\hline$a f$ & & 53.m03696 & ВС003065 & AAM61706 & AY061049 & XM_327865 & AL031535 & Pho85p \\
\hline$a o$ & & 20147.m00190 & & & & & & \\
\hline & & & & & & & & \\
\hline$a n$ & PhoB & AN4310.1 & $6,00 \mathrm{E}-18$ & & $3,00 \mathrm{E}-18$ & 0 & & $8,00 \mathrm{E}-88$ \\
\hline$a f$ & & 58.m09009 & BX537917 & & NM_175928 & NUC-2 & & Pho81p \\
\hline ao & & 20147.m00190 & & & & & & \\
\hline & & & & & & & & \\
\hline$a n$ & SrbA & AN2489.1 & $8,00 \mathrm{E}-56$ & & $5,00 \mathrm{E}-53$ & $1,00 \mathrm{E}-113$ & 0 & $6,00 \mathrm{E}-81$ \\
\hline$a f$ & & 59.m08500 & NM_015076 & & NM_080487 & BX294028 & Z98977 & Srb10p \\
\hline$a o$ & & 20149.m00296 & CDK8 & & CDK8 & & & \\
\hline & & & & & & & & \\
\hline$a n$ & SrbB & AN2172.1 & $3,00 \mathrm{E}-19$ & $5,00 \mathrm{E}-15$ & $6,00 \mathrm{E}-21$ & $8,00 \mathrm{E}-61$ & $1,00 \mathrm{E}-16$ & $6,00 \mathrm{E}-43$ \\
\hline$a f$ & & 72.m19668 & NM_005190 & NM_124239 & AE003706 & AL355930 & AL035085 & Srb11p \\
\hline ao & & 20180.m00841 & CCNC & & & & & \\
\hline & & & & & & & & \\
\hline an & SkpA (SconC) & $\begin{array}{r}10038 . \mathrm{m} 00099 \\
\end{array}$ & $\begin{array}{c}\mathrm{e}-41 \\
\end{array}$ & & e-34 & $\begin{array}{c}\text { e-49 } \\
\text { XP3231383 }\end{array}$ & & $\begin{array}{c}\mathrm{e}-36 \\
\mathrm{~A}-36 \mathrm{C}\end{array}$ \\
\hline$\frac{a f}{a o}$ & & $\begin{array}{c}\text { 71.m15951 } \\
\text { 20108.m00127 }\end{array}$ & $\begin{array}{c}\text { NP_73377 } \\
\text { SKP1 }\end{array}$ & $\begin{array}{c}\text { AAK26104 } \\
\text { ASK10 }\end{array}$ & NP_477390 & XP331383 & $\begin{array}{c}\text { NP_595455 } \\
\text { SKP1 }\end{array}$ & $\begin{array}{c}\text { AAC49492 } \\
\text { Skplp }\end{array}$ \\
\hline & & & & & & & & \\
\hline$a n$ & CulA & 10015.m00090 & 0 & e- 83 & e-162 & 0 & e-176 & e-116 \\
\hline$\overline{a f}$ & & $70 . \mathrm{m} 15237$ & NP_003583 & NP_567243 & NP_523655 & XP_324561 & NP_594259 & NP_010150 \\
\hline$a o$ & & 20180.m01194 & CUL1 & CUL1 & $\operatorname{lin} 19$ & & pcu1 & Cdc53p \\
\hline & & & & & & & & \\
\hline$a n$ & HrtA & 10163.m00039 & e-43 & $\mathrm{e}-40$ & $\mathrm{e}-40$ & $e-43$ & $\mathrm{e}-36$ & e-29 \\
\hline$a f$ & & 71.m15978 & NP_055063 & NP_189869 & NP_569852 & XP_326079 & NP_593388 & NP_014508 \\
\hline$a o$ & & $20108 . \mathrm{m} 00096$ & RBX1 & ROC1 & ROC1A & & RBX1 & Hrtlp \\
\hline$a n$ & CdcD & AN5517.1 & $4,00 \mathrm{E}-66$ & & $1,00 \mathrm{E}-66$ & 0 & $6,00 \mathrm{E}-104$ & $1,00 \mathrm{E}-77$ \\
\hline$a f$ & & 69.m15442 & NM_033632 & & NM_168073 & XM_325793 & Z98602 & $\mathrm{Cdc} 4 \mathrm{p}$ \\
\hline$a o$ & & 20178.m00790 & FBW7 & & ago & & & \\
\hline & & & & & & & & \\
\hline$a n$ & SprA & $\begin{array}{ll}\mathrm{AN} 2142.1 \\
\end{array}$ & $7,00 \mathrm{E}-145$ & $7,00 \mathrm{E}-145$ & & 0 & $1,00 \mathrm{E}-175$ & $2,00 \mathrm{E}-151$ \\
\hline$a f$ & & 72.m19699 & ВТ009843 & Impa-4 & & XM_326741 & AL031323 & Sprlp \\
\hline$a o$ & & AO070343000188 & & Y14616 & & & & \\
\hline & & & & & & & & \\
\hline$a n$ & KapA & $\begin{array}{l}\text { AN0906.1 } \\
\end{array}$ & $1,00 \mathrm{E}-135$ & 0 & 0 & 0 & 0 & $1,00 \mathrm{E}-149$ \\
\hline$a f$ & & $70 . \mathrm{m} 15515$ & ВТ009797 & AK117217 & Ketel & XM_329200 & Z98532 & Кар95p \\
\hline ao & & AO070320000082 & & & AJ002729 & & & \\
\hline$a n$ & TapA & AN0120.1 & $300 \mathrm{~F}_{0} 07$ & $600 \mathrm{~F}_{-00}$ & $600 \mathrm{~F}_{-05}$ & 100F-52 & $400 F_{-26}$ & $500 \mathrm{E}-23$ \\
\hline$a f$ & 1 aps & 71.m15400 & NM_001551 & BX831385 & NM_165039 & 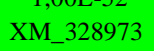 & AL049522 & Tap42p \\
\hline ao & & $10004 . \mathrm{m} 00070$ & & & & & & \\
\hline & & & & & & & & \\
\hline$a n$ & TorA & $\begin{array}{l}\text { AN5982.1 } \\
\end{array}$ & 0 & 0 & 0 & 0 & 0 & 0 \\
\hline af & & 72.m19942 & FRAP1 & TOR-1 & Tor & XM_325462 & AL049558 & Tor1p \\
\hline$a o$ & & 20177.m00675 & NM_004958 & AF178967 & NM_080152 & & & \\
\hline
\end{tabular}




\begin{tabular}{|c|c|c|c|c|c|c|c|c|}
\hline & Protein & Coding sequence & H. sapiens & A. thaliana & D. & N. crassa & S. pombe & S. cerevisiae \\
\hline$a n$ & \multirow[t]{3}{*}{ GapA } & $\begin{array}{l}\text { AN5678.1 } \\
\end{array}$ & & & & \multirow{3}{*}{$\begin{array}{c}1,00 \mathrm{E}-171 \\
\text { XM_325684 }\end{array}$} & \multirow{3}{*}{$\begin{array}{l}2,00 \mathrm{E}-124 \\
\text { AL353014 }\end{array}$} & \multirow{3}{*}{$\begin{array}{c}1,00 \mathrm{E}-137 \\
\text { Gap1p }\end{array}$} \\
\hline$a f$ & & 65.m07404 & & & & & & \\
\hline ao & & 20146.m00246 & & & & & & \\
\hline & & & & & & & & \\
\hline$a n$ & \multirow[t]{3}{*}{ PutD } & 20127.m00101 & & & & \multirow{3}{*}{$\begin{array}{c}4,00 \mathrm{E}-94 \\
\mathrm{XM} \_327414\end{array}$} & \multirow{3}{*}{$\begin{array}{c}\text { 7,00E-77 } \\
\text { AL132779 }\end{array}$} & \multirow{3}{*}{$\begin{array}{c}1,00 \mathrm{E}-73 \\
\text { Put } 4 p\end{array}$} \\
\hline$a f$ & & $56 . \mathrm{m} 02346$ & & & & & & \\
\hline ao & & 20127.m00101 & & & & & & \\
\hline & \multirow{4}{*}{ NprA } & & & & & \multirow{4}{*}{$\begin{array}{c}1,00 \mathrm{E}-135 \\
\text { XM_323687 }\end{array}$} & \multirow{4}{*}{$\begin{array}{c}2,00 \mathrm{E}-97 \\
\text { AL023518 }\end{array}$} & \multirow{4}{*}{$\begin{array}{l}2,00 \mathrm{E}-75 \\
\text { Nprlp }\end{array}$} \\
\hline$a n$ & & AN0115.1 & & & & & & \\
\hline$a f$ & & 71.m15394 & & & & & & \\
\hline ao & & 20148.m00245 & & & & & & \\
\hline & & & & & & & & \\
\hline$a n$ & \multirow[t]{3}{*}{ SlcA } & AN6519.1 & $2,00 \mathrm{E}-62$ & & $1,00 \mathrm{E}-60$ & 0 & & 1 -e29 \\
\hline$a f$ & & 62.m03107 & SLC7 & & NP_730005 & XM_323115 & & Mup1p \\
\hline ao & & 20107.m00080 & LAT1 & & & & & \\
\hline & & & & & & & & \\
\hline$a n$ & SlcB & AN6782.1 & $5,00 \mathrm{E}-24$ & & $2,00 \mathrm{E}-18$ & M. grisea & & \\
\hline$a f$ & & - & EAA1 & & NP_477427 & $9,00 \mathrm{E}-80$ & & \\
\hline$a o$ & & - & & & & XM_367728 & & \\
\hline & & & & & & & & \\
\hline$a n$ & SlcC & AN4428.1 & $2,00 \mathrm{E}-31$ & $2,00 \mathrm{E}-28$ & $5,00 \mathrm{E}-57$ & $1,00 \mathrm{E}-163$ & $1,00 \mathrm{E}-25$ & $4,00 \mathrm{E}-96$ \\
\hline$a f$ & & 58.m09004 & FLJ39822 & AY149936 & NM_136600 & XM_367253 & CAA20055 & Avt2p \\
\hline ao & & $20110 . \mathrm{m} 00133$ & (SCL36?) & & & & & \\
\hline & & & & & & & & \\
\hline$a n$ & SlcD & AN8966.1 & $\begin{array}{c}9,00 \mathrm{E}-49 \\
\text { System Beta, }\end{array}$ & & $\begin{array}{c}2,00 \mathrm{E}-47 \\
\text { SerT }\end{array}$ & & & \\
\hline$a f$ & & - & & & NM_079122 & & & \\
\hline ao & & 20170.m00328 & P30531 & & & EAA54090 & & \\
\hline & & & & & & & & \\
\hline$a n$ & SlcE & AN4477.1 & $2,00 \mathrm{E}-30$ & $1,00 \mathrm{E}-27$ & $2,00 \mathrm{E}-32$ & 0 & $1,00 \mathrm{E}-130$ & $2,00 \mathrm{E}-112$ \\
\hline$a f$ & & 58.m07843 & SLC36 & BX828060 & AY119064 & XM_323083 & Z68144 & Avt3p \\
\hline ao & & 20142.m00243 & & & & & & \\
\hline$a n$ & SlcF & AN7777.1 & $1.00 \mathrm{E}-30$ & $500 \mathrm{E}-24$ & $200 \mathrm{E}-28$ & $100 \mathrm{~F}-144$ & $300 \mathrm{E}-95$ & $100 \mathrm{E}-91$ \\
\hline$a f$ & & 57.m05337 & SLC36 & BX827846 & NM 206319 & XM 325629 & Z68144 & Avt $3 p$ \\
\hline ao & & 20123.m00162 & & & & & & \\
\hline & & & & & & & & \\
\hline$a n$ & HisA & $\begin{array}{ll}\text { AN3748.1 } \\
\end{array}$ & & & & $1,00 \mathrm{E}-96$ & $1,00 \mathrm{E}-79$ & $1,00 \mathrm{E}-86$ \\
\hline$a f$ & EC 2.4.2.17 & $65 . \mathrm{m} 07383$ & & & & XM_332011 & his1 & His1p \\
\hline ao & & 20146.m00225 & & & & & & \\
\hline & & & & & & & & \\
\hline$a n$ & HisB & $\begin{array}{l}\text { AN6536.1 } \\
\end{array}$ & & & & $8,00 \mathrm{E}-55$ & $5,00 \mathrm{E}-48$ & $5,00 \mathrm{E}-51$ \\
\hline$a f$ & EC 4..2.1.19 & 62.m03136 & & & & XM_326792 & AL023286 & His3p \\
\hline ao & & 20107.m00111 & & & & & His5 & \\
\hline & & & & & & & & \\
\hline$a n$ & HisC & $\begin{array}{l}\text { AN0797.1 } \\
\end{array}$ & & $1,00 \mathrm{E}-106$ & & 0 & $4,00 \mathrm{E}-117$ & 0 \\
\hline$a f$ & EC3.6.1.31, & 70.m15398 & & BX830420 & & his-3 & AL353012 & His $4 p$ \\
\hline & EC1.1.1.23 & & & & & & & \\
\hline ao & & 20076.m00051 & & & & & & \\
\hline & & & & & & & & \\
\hline$a n$ & HisD & AN7044.1 & & & & $5,00 \mathrm{E}-76$ & $2,00 \mathrm{E}-58$ & $2,00 \mathrm{E}-38$ \\
\hline$a f$ & EC 3.1.3.15 & 89.m02040 & & & & XM_327259 & AL031324 & His $2 p$ \\
\hline ao & & 20119.m00140 & & & & & & \\
\hline & & & & & & & & \\
\hline$a n$ & HisE & AN0717.1 & & & & $4,00 \mathrm{E}-154$ & $2,00 \mathrm{E}-112$ & $3,00 \mathrm{E}-85$ \\
\hline$a f$ & EC 2.6.1.9 & 70.m15350 & & & & XM_326214 & his3 & His5p \\
\hline ao & & 20180.m01047 & & & & & & \\
\hline & & & & & & & & \\
\hline$a n$ & HisHF & AN7430.1 & & $7,00 \mathrm{E}-137$ & & 0 & $2,00 \mathrm{E}-176$ & 0 \\
\hline$a f$ & EC 5.3.1.16, & 20066.m00025 & & hisHF & & XM_327441 & AL033388 & His7p \\
\hline ao & EC 2.4.2.- & 20066.m00025 & & & & & & \\
\hline & & & & & & & & \\
\hline$a n$ & LysA & $\begin{array}{l}\text { AN2873.1 } \\
\end{array}$ & & & $2,00 \mathrm{E}-04$ & $2,00 \mathrm{E}-109$ & $1,00 \mathrm{E}-101$ & $2,00 \mathrm{E}-98$ \\
\hline$a f$ & EC 1.5.1.7 & 59.m08722 & & & AAR82744 & LYS-1 & lys 3 & Lys1p \\
\hline ao & & 20175.m00452 & & & & & & \\
\hline
\end{tabular}


Chapter 3

\begin{tabular}{|c|c|c|c|c|c|c|c|c|}
\hline & Protein & Coding sequence & H. sapiens & A. thaliana & $D$. & N. crassa & S. pombe & S. cerevisiae \\
\hline an & \multirow{3}{*}{$\begin{array}{c}\text { LysB } \\
\text { EC 1.2.1.31 }\end{array}$} & AN5610.1 & & & & \multirow{3}{*}{$\begin{array}{c}0 \\
\text { CAB97293 }\end{array}$} & \multirow{3}{*}{$\begin{array}{c}0 \\
\text { lys1 }\end{array}$} & \multirow{3}{*}{$\begin{array}{c}0 \\
\text { Lys } 2 p\end{array}$} \\
\hline$a f$ & & 58.m07534 & & & & & & \\
\hline ao & & 20138.m00117 & & & & & & \\
\hline & & & & & & & & \\
\hline an & \multirow{3}{*}{$\begin{array}{c}\text { LysD } \\
\text { EC 2.6.1.39 }\end{array}$} & AN8519.1 & \multirow{3}{*}{$\begin{array}{l}7,00 \mathrm{E}-51 \\
\text { AADAT }\end{array}$} & & & \multirow{3}{*}{$\begin{array}{c}9,00 \mathrm{E}-27 \\
\text { XP_331508 }\end{array}$} & \multirow{3}{*}{$\begin{array}{c}1,00 \mathrm{E}-39 \\
\text { CAA21918 }\end{array}$} & \multirow{3}{*}{$\begin{array}{c}1,00 \mathrm{E}-31 \\
\text { Aro8p? }\end{array}$} \\
\hline$a f$ & & manual annotation & & & & & & \\
\hline ao & & manual annotation & & & & & & \\
\hline & \multirow{4}{*}{$\begin{array}{c}\text { LysE } \\
\text { EC 1.1.1.155 }\end{array}$} & & \multirow{4}{*}{$\begin{array}{c}\text { 6,00E-40 } \\
\text { NP_005521 }\end{array}$} & & & & & \\
\hline$a n$ & & AN5206.1 & & $2,00 \mathrm{E}-42$ & $2,00 \mathrm{E}-40$ & $5,00 \mathrm{E}-116$ & $2,00 \mathrm{E}-89$ & $3,00 \mathrm{E}-80$ \\
\hline$a f$ & & 69.m14903 & & NP_850549 & Q9VWH4 & CAE76248 & NP_594004 & Lys $12 p$ \\
\hline ao & & 20037.m00016 & & & & & & \\
\hline & & & & & & & & \\
\hline an & LysF & AN6521.1 & $1,00 \mathrm{E}-34$ & $2,00 \mathrm{E}-37$ & $1,00 \mathrm{E}-34$ & 0 & 0 & 0 \\
\hline$a f$ & EC 4.2.1.36 & 71.m15675 & AAD19351 & NP_567405 & CAB93521 & CAD71225 & NP_593437 & Lys $4 p$ \\
\hline ao & & 20107.m00082 & & & & & & \\
\hline & & & & & & & & \\
\hline an & LysG & AN5601.1 & $4,00 \mathrm{E}-71$ & $9,00 \mathrm{E}-97$ & $2,00 \mathrm{E}-54$ & $2,00 \mathrm{E}-148$ & $7,00 \mathrm{E}-128$ & $2,00 \mathrm{E}-133$ \\
\hline$a f$ & EC 1.5.1.10 & 58.m07525 & AAF03526 & AAD00700 & AAR82744 & CAC28679 & NP_596411 & Lys9p \\
\hline$a o$ & & 20165.m00188 & & & & & & \\
\hline & & & & & & & & \\
\hline$a n$ & AcvA & manual annotation & & & & & & \\
\hline$a f$ & & & & & & & & \\
\hline$a o$ & & 20169.m00348 & & & & & & \\
\hline & & & & & & & & \\
\hline$a n$ & IpnA & AN2622.1 & & & & & & \\
\hline$a f$ & & & & & & & & \\
\hline$a o$ & & 20169.m00349 & & & & & & \\
\hline & & & & & & & & \\
\hline$a n$ & AatA & AN2623.1 & & & & & & \\
\hline$a f$ & & & & & & & & \\
\hline ao & & 20169.m00350 & & & & & & \\
\hline & & & & & & & & \\
\hline$a n$ & TrpA & AN3695.1 & & & & $1,00 \mathrm{E}-116$ & $1,00 \mathrm{E}-123$ & $2,00 \mathrm{E}-134$ \\
\hline$a f$ & EC 4.1.3.27 & 69.m15404 & & & & AL031966 & BX284763 & Trp2p \\
\hline$a o$ & & 20178.m00733 & & & & & & \\
\hline & & & & & & & & \\
\hline an & TrpB & AN6231.1 & & & & 0 & 0 & 0 \\
\hline$a f$ & EC 4.2.1.20 & 72.m19978 & & & & $\operatorname{trp}-3$ & Z98974 & $\operatorname{Trp5p}$ \\
\hline ao & & 20141.m00194 & & & & & & \\
\hline & & & & & & & & \\
\hline$a n$ & TrpC & 20180.m01178 & & & & 0 & 0 & $2,00 \mathrm{E}-147$ \\
\hline$a f$ & EC 4.1.1.48 & 70.m15251 & & & & trp-1 & trp-1 & Trp3p \\
\hline ao & & 20180.m01178 & & & & & & \\
\hline & & & & & & & & \\
\hline$a n$ & TrpD & AN3634.1 & & & & $2,00 \mathrm{E}-97$ & $2,00 \mathrm{E}-41$ & $2,00 \mathrm{E}-37$ \\
\hline$a f$ & EC 2.4.2.18 & $58 . \mathrm{m} 07467$ & & & & XM_323763 & AL023554 & $\operatorname{Trp} 4 p$ \\
\hline ao & & $20165 . \mathrm{m} 00263$ & & & & & & \\
\hline & & & & & & & & \\
\hline$a n$ & AroC & AN6866.1 & & & & $6,00 \mathrm{E}-97$ & $1,00 \mathrm{E}-51$ & $7,00 \mathrm{E}-52$ \\
\hline$a f$ & EC 5.4.99.5 & 71.m15266 & & & & & & Aro7p \\
\hline ao & & 20151.m00232 & & & & & & \\
\hline & & & & & & & & \\
\hline an & TyrA & AN5959.1 & & & & 9,00E-96 & $7,00 \mathrm{E}-94$ & $2,00 \mathrm{E}-87$ \\
\hline$a f$ & EC 1.3.1.13 & 72.m19175 & & & & XM_322553 & AL023776 & Tyr1p \\
\hline ao & & 20177.m00688 & & & & & & \\
\hline & & & & & & & & \\
\hline$a n$ & AroH & AN6338.1 & $1,00 \mathrm{E}-54$ & & & $1,00 \mathrm{E}-151$ & $1,00 \mathrm{E}-105$ & $8,00 \mathrm{E}-99$ \\
\hline$a f$ & EC 2.6.1.57 & 72.m19971 & ВС031068 & & & XM_331507 & Z99261 & Aro8p \\
\hline ao & & 20073.m00044 & & & & & & \\
\hline & & & & & & & & \\
\hline$a n$ & AroH & AN6338.1 & $1,00 \mathrm{E}-54$ & & & $1,00 \mathrm{E}-151$ & $1,00 \mathrm{E}-105$ & $8,00 \mathrm{E}-99$ \\
\hline$a f$ & EC 2.6.1.57 & 72.m19971 & ВC031069 & & & XM_331508 & Z99262 & Aro8p \\
\hline ao & & 20073.m00044 & & & & & & \\
\hline & & & & & & & & \\
\hline$a n$ & PhaA & manual annotation & & & $5,00 \mathrm{E}-31$ & $1,00 \mathrm{E}-47$ & $1,00 \mathrm{E}-31$ & $9,00 \mathrm{E}-32$ \\
\hline$a f$ & & $71 . \mathrm{m} 15987$ & & & NM_202520 & XM_322494 & CAB10811 & Pha2p \\
\hline$a o$ & & 20108.m00087 & & & & & & \\
\hline
\end{tabular}


Table 2.: Proteins found among all investigated organisms and average homology over all proteins to A. nidulans

\begin{tabular}{|c|c|c|c|c|c|c|c|}
\hline Protein & Putative protein function & N. crassa & S. cerevisiae & S. pombe & A. thaliana & H. sapiens & D. melanogaster \\
\hline $\mathrm{CpcC}$ & Sensor kinase of $g c / c p c$ & $\begin{array}{c}0 \\
\text { Gcn } 2 p\end{array}$ & $\begin{array}{c}0 \\
\text { Gcn2p }\end{array}$ & $\begin{array}{l}1,00 \mathrm{E}-154 \\
\text { CPC-3 }\end{array}$ & $\begin{array}{l}2,00 \mathrm{E}-58 \\
\text { Gcn } 2 \mathrm{p}\end{array}$ & $\begin{array}{c}0 \\
\text { eIF2a kinase }\end{array}$ & $\begin{array}{c}2,00 \mathrm{E}-76 \\
\text { eIF2a kinase }\end{array}$ \\
\hline $\mathrm{CpcB}$ & transcription repressor of $\mathrm{CpcA}$ & $\begin{array}{c}1,00 \mathrm{E}-177 \\
\text { CPC-2 }\end{array}$ & $\begin{array}{c}3,00 \mathrm{E}-98 \\
\mathrm{Cpc} 2 \mathrm{p}\end{array}$ & $\begin{array}{c}1,00 \mathrm{E}-136 \\
\mathrm{Cpc} 2\end{array}$ & \begin{tabular}{|c|}
$1,00 \mathrm{E}-118$ \\
AAL34190.1
\end{tabular} & $\begin{array}{c}\text { 1,00E-139 } \\
\text { GNB2L1 }\end{array}$ & $\begin{array}{c}1,00 \mathrm{E}-129 \\
\text { Rack1 }\end{array}$ \\
\hline $\mathrm{eIF} 2 \mathrm{~b}$ & Translation machinery & $\begin{array}{l}\text { 2,00E-91 } \\
\text { EIF2 beta }\end{array}$ & $\begin{array}{l}3,00 \mathrm{E}-62 \\
\text { EIF2 beta }\end{array}$ & $\begin{array}{l}5,00 \mathrm{E}-63 \\
\text { EIF2 beta }\end{array}$ & $\begin{array}{l}2,00 \mathrm{E}-46 \\
\text { EIF2 beta }\end{array}$ & $\begin{array}{l}\text { 3,00E-50 } \\
\text { EIF2beta }\end{array}$ & $\begin{array}{l}2,00 \mathrm{E}-47 \\
\text { EIF2 beta }\end{array}$ \\
\hline $\mathrm{eIF} 2 \alpha$ & Translation machinery & $\begin{array}{l}\text { 4,00E-118 } \\
\text { EIF2 alpha }\end{array}$ & $\begin{array}{c}4,00 \mathrm{E}-95 \\
\text { EIF2 alpha } \\
\text { Gcn3p }\end{array}$ & $\begin{array}{c}\text { 3,00E-97 } \\
\text { EIF2 alpha }\end{array}$ & $\begin{array}{c}1,00 \text { E-72 } \\
\text { EIF2 alpha }\end{array}$ & $\begin{array}{c}4,00 \mathrm{E}-71 \\
\text { EIF2 alpha }\end{array}$ & $\begin{array}{c}2,00 \text { E-65 } \\
\text { EIF2 alpha }\end{array}$ \\
\hline eIF $2 \gamma$ & Translation machinery & $\begin{array}{c}0 \\
\text { EIF2 gamma } \\
\end{array}$ & $\begin{array}{c}0 \\
\text { Gcd11p } \\
\end{array}$ & \begin{tabular}{|c|}
0 \\
EIF2 gamma \\
\end{tabular} & \begin{tabular}{|c|}
$4,00 \mathrm{E}-173$ \\
EIF2 gamma
\end{tabular} & $\begin{array}{c}0 \\
\text { EIF2 gamma } \\
\end{array}$ & $\begin{array}{c}0 \\
\text { EIF2 gamma }\end{array}$ \\
\hline eIF2-B $\delta$ & Translation machinery & $\begin{array}{c}2,00 \mathrm{E}-73 \\
\text { CAE76139 }\end{array}$ & $\begin{array}{c}1,00 \mathrm{E}-79 \\
\mathrm{Gcd} 2 \mathrm{p}\end{array}$ & $\begin{array}{c}1,00 \mathrm{E}-89 \\
\text { CAA91965 }\end{array}$ & $\begin{array}{c}1,00 \mathrm{E}-71 \\
\text { AAC23414 }\end{array}$ & $\begin{array}{c}5,00 \mathrm{E}-76 \\
\text { EIF2B4 }\end{array}$ & $\begin{array}{c}5,00 \mathrm{E}-62 \\
\text { EAL25620 }\end{array}$ \\
\hline eIF2-B $\beta$ & Translation machinery & $\begin{array}{c}2,00 \mathrm{E}-66 \\
\text { CAD71011 }\end{array}$ & $\begin{array}{c}1,00 \mathrm{E}-56 \\
\text { Gcd7p }\end{array}$ & $\begin{array}{c}1,00 \mathrm{E}-76 \\
\text { CAB52277 }\end{array}$ & $\begin{array}{c}2,00 \mathrm{E}-58 \\
\text { AAF20216 }\end{array}$ & $\begin{array}{c}\text { 6,00E-53 } \\
\text { EIF2B4 }\end{array}$ & $\begin{array}{c}2,00 \mathrm{E}-44 \\
\text { NP_570020 }\end{array}$ \\
\hline eIF2-B $\alpha$ & Translation machinery & $\begin{array}{c}5,00 \mathrm{E}-52 \\
\text { XP_323697 }\end{array}$ & $\begin{array}{c}2,00 \mathrm{E}-62 \\
\mathrm{Gcn} 3 \mathrm{p}\end{array}$ & $\begin{array}{c}2,00 \mathrm{E}-66 \\
\text { CAB57849 }\end{array}$ & $\begin{array}{c}1,00 \mathrm{E}-46 \\
\mathrm{AAF} 02861\end{array}$ & $\begin{array}{l}1,00 \mathrm{E}-46 \\
\text { EIF2B1 }\end{array}$ & $\begin{array}{c}6,00 \mathrm{E}-42 \\
\text { AAG38014 }\end{array}$ \\
\hline eIF2-B $\varepsilon$ & Translation machinery & $\begin{array}{c}7,00 \mathrm{E}-123 \\
\text { XP_331190 } \\
\end{array}$ & $\begin{array}{c}\text { 6,00E-101 } \\
\text { Gcd6p }\end{array}$ & $\begin{array}{c}5,00 \mathrm{E}-129 \\
\text { Tif225 } \\
\end{array}$ & $\begin{array}{c}2,00 \mathrm{E}-99 \\
\text { CAB78832 } \\
\end{array}$ & $\begin{array}{l}2,00 \mathrm{E}-91 \\
\text { eIF2-B e }\end{array}$ & $\begin{array}{c}1,00 \mathrm{E}-60 \\
\text { AAG38017 }\end{array}$ \\
\hline $\mathrm{Gcn} A$ & CpcC mediator & $\begin{array}{c}0 \\
\text { XP_325658 }\end{array}$ & $\begin{array}{c}0 \\
\text { Gcn1p }\end{array}$ & $\begin{array}{c}0 \\
\text { CAA92385 }\end{array}$ & $\begin{array}{c}0 \\
\text { NP_176659 }\end{array}$ & $\begin{array}{c}0 \\
\text { HsGCN1 }\end{array}$ & $\begin{array}{c}0 \\
\text { EAA46127 }\end{array}$ \\
\hline eIF3a & Translation machinery & $\begin{array}{c}0.0 \\
\text { XP_322126 }\end{array}$ & $\begin{array}{c}\text { E-65 } \\
\text { NP_009635 } \\
\text { Tif32p }\end{array}$ & $\begin{array}{c}\text { E-135 } \\
\text { NP_596379 } \\
\text { eIF3p110 } \\
\end{array}$ & $\begin{array}{c}\text { E-83 } \\
\text { NP_192881 } \\
\text { elF3a } \\
\end{array}$ & $\begin{array}{c}\text { E-97 } \\
\text { NP_003741 } \\
\text { eIF3a S10 }\end{array}$ & $\begin{array}{c}\text { E-78 } \\
\text { NP_649470 } \\
\text { eIF3 S10 }\end{array}$ \\
\hline eIF3b & Translation machinery & $\begin{array}{c}0.0 \\
\text { XP_330984 }\end{array}$ & $\begin{array}{c}\text { E-121 } \\
\text { NP_015006 } \\
\text { Prt1p }\end{array}$ & $\begin{array}{c}\text { E-169 } \\
\text { NP_594528 } \\
\text { 3 beta }\end{array}$ & $\begin{array}{c}\text { E-117 } \\
\text { NP_568498 } \\
\text { elF3b }\end{array}$ & $\begin{array}{c}\text { E-130 } \\
\text { NP_874371 } \\
\text { eIF3b S9 }\end{array}$ & $\begin{array}{c}\text { E-112 } \\
\text { NP_611228 } \\
\text { eIF3 S9 }\end{array}$ \\
\hline eIF3c & Translation machinery & $\begin{array}{c}\text { E-121 } \\
\text { XP_328537 }\end{array}$ & $\begin{array}{c}\text { E-104 } \\
\text { NP_014040 } \\
\text { Nip1p }\end{array}$ & $\begin{array}{c}\text { E-143 } \\
\text { NP_593828 } \\
\text { eIF3 p98 }\end{array}$ & $\begin{array}{c}\text { E-72 } \\
\text { AAC83464 } \\
\text { eIF3c }\end{array}$ & $\begin{array}{c}\text { E-75 } \\
\text { AAC27674 } \\
\text { eIF3c S8 }\end{array}$ & $\begin{array}{c}\text { E-65 } \\
\text { NP_611242 } \\
\text { elF3 S8 }\end{array}$ \\
\hline elF3i & Translation machinery & $\begin{array}{c}\text { E-143 } \\
\text { XP_323195 }\end{array}$ & $\begin{array}{c}\text { E-104 } \\
\text { NP_013866 } \\
\text { Tif34p }\end{array}$ & $\begin{array}{c}\text { E-120 } \\
\text { NP_594958 } \\
\text { eIF3 p39 }\end{array}$ & $\begin{array}{c}\text { E-73 } \\
\text { NP_182152 } \\
\text { elF3i }\end{array}$ & $\begin{array}{c}\text { E-99 } \\
\text { NP_003748 } \\
\text { eIF3i S2 }\end{array}$ & $\begin{array}{c}\text { E-84 } \\
\text { NP_523478 } \\
\text { TRIP1 }\end{array}$ \\
\hline PhoA & CpcA degradation & $\begin{array}{c}5,00 \mathrm{E}-143 \\
\text { XM_327865 }\end{array}$ & $\begin{array}{c}2,00 \mathrm{E}-109 \\
\text { Pho85p }\end{array}$ & $\begin{array}{l}4,00 \mathrm{E}-115 \\
\text { AL031535 }\end{array}$ & $\begin{array}{c}3,00 \mathrm{E}-93 \\
\text { AAM61706 }\end{array}$ & $\begin{array}{c}1,00 \mathrm{E}-97 \\
\text { BC003065 }\end{array}$ & $\begin{array}{c}1,00 \mathrm{E}-97 \\
\text { AY061049 }\end{array}$ \\
\hline SrbB & CpcA degradation & $\begin{array}{c}8,00 \mathrm{E}-61 \\
\text { AL355930 }\end{array}$ & $\begin{array}{c}\text { 6,00E-43 } \\
\text { Srb11p }\end{array}$ & $\begin{array}{c}1,00 \mathrm{E}-16 \\
\text { AL035085 }\end{array}$ & $\begin{array}{c}5,00 \mathrm{E}-15 \\
\text { NM_124239 }\end{array}$ & $\begin{array}{c}3,00 \mathrm{E}-19 \\
\mathrm{NM} \_005190 \\
\text { CCNC }\end{array}$ & $\begin{array}{c}6,00 \mathrm{E}-21 \\
\text { AE003706 }\end{array}$ \\
\hline $\begin{array}{c}\text { SkpA } \\
\text { (SconC) }\end{array}$ & SCF compound & $\begin{array}{c}\text { E-49 } \\
\text { XP331383 }\end{array}$ & $\begin{array}{c}\text { E-36 } \\
\text { AAC49492 } \\
\text { Skplp }\end{array}$ & $\begin{array}{c}\text { E-48 } \\
\text { NP_595455 } \\
\text { SKP1 }\end{array}$ & $\begin{array}{c}\text { E-37 } \\
\text { AAK26104 } \\
\text { ASK10 }\end{array}$ & $\begin{array}{c}\text { E-41 } \\
\text { NP_73377 } \\
\text { SKP1 }\end{array}$ & $\begin{array}{c}\text { E-34 } \\
\text { NP_477390 }\end{array}$ \\
\hline CulA & SCF compound & $\begin{array}{c}0 \\
\text { XP_324561 }\end{array}$ & $\begin{array}{c}\text { E-116 } \\
\text { NP_010150 } \\
\text { Cdc53p }\end{array}$ & \begin{tabular}{|c|} 
E-176 \\
NP_594259 \\
pcu1 \\
\end{tabular} & $\begin{array}{c}\text { E-83 } \\
\text { NP_567243 } \\
\text { CUL1 }\end{array}$ & $\begin{array}{c}0 \\
\text { NP_003583 } \\
\text { CUL1 } \\
\end{array}$ & $\begin{array}{c}\text { E-162 } \\
\text { NP_523655 } \\
\text { lin19 }\end{array}$ \\
\hline HrtA & SCF compound & $\begin{array}{c}\text { E-43 } \\
\text { XP_326079 }\end{array}$ & $\begin{array}{c}\text { E-29 } \\
\text { NP_014508 } \\
\text { Hrt1p }\end{array}$ & $\begin{array}{c}\text { E-36 } \\
\text { NP_593388 } \\
\text { RBX1 }\end{array}$ & $\begin{array}{c}\text { E-40 } \\
\text { NP_189869 } \\
\text { ROC1 }\end{array}$ & $\begin{array}{c}\text { E-43 } \\
\text { NP_055063 } \\
\text { RBX1 }\end{array}$ & $\begin{array}{c}\text { E-40 } \\
\text { NP_569852 } \\
\text { ROC1A }\end{array}$ \\
\hline KapA & CpcA transport & $\begin{array}{c}0 \\
\text { XM_329200 }\end{array}$ & $\begin{array}{l}1,00 \mathrm{E}-149 \\
\text { Kap95p }\end{array}$ & $\begin{array}{c}0 \\
\text { Z98532 }\end{array}$ & $\begin{array}{c}0 \\
\text { AK117217 }\end{array}$ & $\begin{array}{l}1,00 \mathrm{E}-135 \\
\text { BT009797 }\end{array}$ & $\begin{array}{c}0 \\
\text { Ketel }\end{array}$ \\
\hline TapA & TOR mediator & $\begin{array}{c}1,00 \text { E-52 } \\
\text { XM_328973 }\end{array}$ & $\begin{array}{l}5,00 \mathrm{E}-23 \\
\text { Tap42p }\end{array}$ & $\begin{array}{c}4,00 \mathrm{E}-26 \\
\text { AL049522 }\end{array}$ & $\begin{array}{c}6,00 \mathrm{E}-09 \\
\text { BX831385 }\end{array}$ & $\begin{array}{c}\text { 3,00E-07 } \\
\text { NM_001551 }\end{array}$ & $\begin{array}{c}6,00 \mathrm{E}-05 \\
\mathrm{NM} \_165039\end{array}$ \\
\hline TorA & Sensor kinase of $\mathrm{gc} / \mathrm{cpc}$ & $\begin{array}{c}0 \\
\text { XM_325462 }\end{array}$ & $\begin{array}{c}0 \\
\text { Torlp }\end{array}$ & $\begin{array}{c}0 \\
\text { AL049558 }\end{array}$ & $\begin{array}{c}0 \\
\text { TOR-1 } \\
\text { AF178967 }\end{array}$ & $\begin{array}{c}0 \\
\text { FRAP1 } \\
\text { NM_004958 }\end{array}$ & $\begin{array}{c}0 \\
\text { Tor } \\
\text { NM_080152 }\end{array}$ \\
\hline SlcF & Amino acid transport & $\begin{array}{c}0 \\
\text { XM_323083 }\end{array}$ & $\begin{array}{c}2,00 \mathrm{E}-112 \\
\text { Avt3p }\end{array}$ & $\begin{array}{c}\text { 1,00E-130 } \\
\text { Z68144 }\end{array}$ & $\begin{array}{c}1,00 \mathrm{E}-27 \\
\text { BX828060 }\end{array}$ & $\begin{array}{r}2,00 \mathrm{E}-30 \\
\text { SLC36A1 }\end{array}$ & $\begin{array}{c}2,00 \mathrm{E}-32 \\
\text { AY119064 }\end{array}$ \\
\hline SlcG & Amino acid transport & $\begin{array}{c}1,00 \mathrm{E}-144 \\
\text { XM_325629 }\end{array}$ & $\begin{array}{c}1,00 \mathrm{E}-91 \\
\text { Avt3p }\end{array}$ & $\begin{array}{c}3,00 \mathrm{E}-95 \\
\text { Z68144 }\end{array}$ & $\begin{array}{c}5,00 \mathrm{E}-24 \\
\mathrm{BX} 827846\end{array}$ & $\begin{array}{r}1,00 \mathrm{E}-30 \\
\text { SLC36A2 }\end{array}$ & $\begin{array}{c}2,00 \mathrm{E}-28 \\
\text { NM_206319 }\end{array}$ \\
\hline $\begin{array}{c}\text { LysE } \\
1.1 .1 .155\end{array}$ & Lysine biosynthesis & $\begin{array}{c}5,00 \mathrm{E}-116 \\
\text { CAE76248 }\end{array}$ & $\begin{array}{c}\text { 3,00E-80 } \\
\text { Lys } 12 p\end{array}$ & $\begin{array}{c}2,00 \mathrm{E}-89 \\
\text { NP_594004 }\end{array}$ & $\begin{array}{c}2,00 \mathrm{E}-42 \\
\text { NP_850549 }\end{array}$ & $\begin{array}{c}6,00 \mathrm{E}-40 \\
\text { NP_005521 }\end{array}$ & $\begin{array}{l}2,00 \mathrm{E}-40 \\
\text { Q9VWH4 }\end{array}$ \\
\hline $\begin{array}{c}\text { LysF } \\
4.2 .1 .36\end{array}$ & Lysine biosynthesis & $\begin{array}{c}0 \\
\text { CAD71225 }\end{array}$ & $\begin{array}{c}0 \\
\text { Lys4p }\end{array}$ & $\begin{array}{c}0 \\
\text { NP_593437 }\end{array}$ & $\begin{array}{c}2,00 \mathrm{E}-37 \\
\text { NP_567405 }\end{array}$ & $\begin{array}{c}1,00 \mathrm{E}-34 \\
\mathrm{AAD} 19351\end{array}$ & $\begin{array}{c}1,00 \mathrm{E}-34 \\
\text { CAB93521 }\end{array}$ \\
\hline $\begin{array}{c}\text { LysG } \\
1.5 .1 .10\end{array}$ & Lysine biosynthesis & $\begin{array}{c}2,00 \mathrm{E}-148 \\
\text { CAC } 28679 \\
\end{array}$ & $\begin{array}{c}2,00 \mathrm{E}-133 \\
\text { Lys9p }\end{array}$ & $\begin{array}{c}7,00 \mathrm{E}-128 \\
\text { NP_596411 } \\
\end{array}$ & $\begin{array}{c}9,00 \mathrm{E}-97 \\
\text { AAD00700 } \\
\end{array}$ & $\begin{array}{c}4,00 \mathrm{E}-71 \\
\text { AAF03526 }\end{array}$ & $\begin{array}{c}2,00 \mathrm{E}-54 \\
\text { AAR82744 }\end{array}$ \\
\hline & Average homology & $2,14 E-53$ & $1,79 E-24$ & $3,57 E-18$ & $2,14 \mathrm{E}-10$ & 1,07E-08 & 2,14E-06 \\
\hline
\end{tabular}


Table 3.: Overview of putative amino acid transporters in A. nidulans

\begin{tabular}{|l|l|}
\hline Transporter & Putative function derived from ortholog function \\
\hline GapA & $\begin{array}{l}\text { General amino acid permease for L-amino acids, some D-amino acids } \\
\text { Trp/Tyr transporter (Jauniaux and Grenson, 1990) }\end{array}$ \\
\hline SlcA & $\begin{array}{l}\text { Sodium independent neutral amino acid transporter (H, M, L, I, V, F, Y, W, } \\
\text { Q), putative amino acid sensor (Padbury } \text { et al., 2004; Verrey } \text { et al., 1999) }\end{array}$ \\
\hline SlcB & $\begin{array}{l}\mathrm{K}^{+} \text {antiport of anionic amino acid transporter (E, D) (Furuta } \text { et al., 2005; } \\
\text { Gonzalez-Gonzalez } \text { et al., 2005) }\end{array}$ \\
\hline SlcC & $\begin{array}{l}\text { Sodium independent neutral amino acid transporter (Q, N, I, L, Y), putative } \\
\text { uptake of amino acids from lysosome (Goberdhan } \text { et al., 2005; Russnak et al., } \\
\text { 2001) }\end{array}$ \\
\hline SlcD & $\begin{array}{l}\text { Cl }{ }^{-} \text {dependent GABA, betaine and taurine transporter, Na }{ }^{+} \text {and Cl }{ }^{-} \text {dependent } \\
\text { high-affinity glycine transporter, Glutamate transporter (Hyde } \text { et al., 2003) }\end{array}$ \\
\hline SlcE & $\begin{array}{l}\text { Sodium independent neutral amino acid transporter (Q, N, I, L, Y), putative } \\
\text { uptake of amino acids from lysosome (Goberdhan } \text { et al., 2005; Russnak et al., } \\
\text { 2001) }\end{array}$ \\
\hline PutD & $\begin{array}{l}\text { Sodium independent neutral amino acid transporter (Q, N, I, L, Y), putative } \\
\text { uptake of amino acids from lysosome (Goberdhan } \text { et al., 2005; Russnak } \text { et al., } \\
\text { 2001) }\end{array}$ \\
\hline
\end{tabular}




\subsection{References}

Abdel-Sater, F., Iraqui, I., Urrestarazu, A., and Andre, B. (2004) The external amino acid signaling pathway promotes activation of Stp1 and Uga35/Dal81 transcription factors for induction of the AGP1 gene in Saccharomyces cerevisiae. Genetics 166: 1727-1739.

Altschul, S.F., Madden, T.L., Schaffer, A.A., Zhang, J., Zhang, Z., Miller, W., and Lipman, D.J. (1997) Gapped BLAST and PSI-BLAST: a new generation of protein database search programs. Nucleic Acids Res 25: 3389-3402.

Andreasson, C., and Ljungdahl, P.O. (2002) Receptor-mediated endoproteolytic activation of two transcription factors in yeast. Genes Dev 16: 3158-3172.

Andreasson, C., Neve, E.P., and Ljungdahl, P.O. (2004) Four permeases import proline and the toxic proline analogue azetidine-2-carboxylate into yeast. Yeast 21: 193-199.

Andrews, P.R., Smith, G.D., and Young, I.G. (1973) Transition-state stabilization and enzymic catalysis. Kinetic and molecular orbital studies of the rearrangement of chorismate to prephenate. Biochemistry 12: 34923498 .

Averous, J., Bruhat, A., Mordier, S., and Fafournoux, P. (2003) Recent advances in the understanding of amino acid regulation of gene expression. $J$ Nutr 133: 2040S-2045S.

Averous, J., Bruhat, A., Jousse, C., Carraro, V., Thiel, G., and Fafournoux, P. (2004) Induction of CHOP expression by amino acid limitation requires both ATF4 expression and ATF2 phosphorylation. $J$ Biol Chem 279: 5288-5297.

Berlanga, J.J., Santoyo, J., and De Haro, C. (1999) Characterization of a mammalian homolog of the GCN2 eukaryotic initiation factor 2alpha kinase. Eur J Biochem 265: 754-762.

Boczko, E.M., Cooper, T.G., Gedeon, T., Mischaikow, K., Murdock, D.G., Pratap, S., and Wells, K.S. (2005) Structure theorems and the dynamics of nitrogen catabolite repression in yeast. Proc Natl Acad Sci U S A 102: $5647-5652$.

Boll, M., Daniel, H., and Gasnier, B. (2004) The SLC36 family: proton-coupled transporters for the absorption of selected amino acids from extracellular and intracellular proteolysis. Pflugers Arch 447: 776-779.

Brakhage, A.A. (1998) Molecular regulation of beta-lactam biosynthesis in filamentous fungi. Microbiol Mol Biol Rev 62: 547-585.

Brakhage, A.A., Sprote, P., Al-Abdallah, Q., Gehrke, A., Plattner, H., and Tuncher, A. (2004) Regulation of penicillin biosynthesis in filamentous fungi. Adv Biochem Eng Biotechnol 88: 45-90.

Brakhage, A.A., Al-Abdallah, Q., Tuncher, A., and Sprote, P. (2005) Evolution of beta-lactam biosynthesis genes and recruitment of trans-acting factors. Phytochemistry 66: 1200-1210.

Braus, G.H. (1991) Aromatic amino acid biosynthesis in the yeast Saccharomyces cerevisiae: a model system for the regulation of a eukaryotic biosynthetic pathway. Microbiol Rev 55: 349-370.

Brüggeman, J., Debets, A.J., Wijngaarden, P.J., deVisser, J.A., and Hoekstra, R.F. (2003) Sex slows down the accumulation of deleterious mutations in the homothallic fungus Aspergillus nidulans. Genetics 164: 479485. 
Bruhat, A., Jousse, C., Wang, X.Z., Ron, D., Ferrara, M., and Fafournoux, P. (1997) Amino acid limitation induces expression of CHOP, a CCAAT/enhancer binding protein-related gene, at both transcriptional and post-transcriptional levels. J Biol Chem 272: 17588-17593.

Busch, S., Hoffmann, B., Valerius, O., Starke, K., Düvel, K., and Braus, G.H. (2001) Regulation of the Aspergillus nidulans hisB gene by histidine starvation. Curr Genet 38: 314-322.

Busch, S., Bode, H.B., Brakhage, A.A., and Braus, G.H. (2003) Impact of the cross-pathway control on the regulation of lysine and penicillin biosynthesis in Aspergillus nidulans. Curr Genet 42: 209-219.

Carsiotis, M., and Jones, R.F. (1974) Cross-pathway regulation: tryptophan-mediated control of histidine and arginine biosynthetic enzymes in Neurospora crassa. J Bacteriol 119: 889-892.

Carsiotis, M., Jones, R.F., and Wesseling, A.C. (1974) Cross-pathway regulation: histidine-mediated control of histidine, tryptophan, and arginine biosynthetic enzymes in Neurospora crassa. J Bacteriol 119: 893-898.

Chaudhuri, J., Chowdhury, D., and Maitra, U. (1999) Distinct functions of eukaryotic translation initiation factors eIF1A and eIF3 in the formation of the $40 \mathrm{~S}$ ribosomal preinitiation complex. J Biol Chem 274: 17975-17980.

Chen, N.H., Reith, M.E., and Quick, M.W. (2004) Synaptic uptake and beyond: the sodium- and chloridedependent neurotransmitter transporter family SLC6. Pflugers Arch 447: 519-531.

Cherkasova, V.A., and Hinnebusch, A.G. (2003) Translational control by TOR and TAP42 through dephosphorylation of eIF2alpha kinase GCN2. Genes Dev 17: 859-872.

Clemens, M.J. (1994) Regulation of eukaryotic protein synthesis by protein kinases that phosphorylate initiation factor eIF-2. Mol Biol Rep 19: 201-210.

Davis, M.A., Askin, M.C., and Hynes, M.J. (2005) Amino acid catabolism by an areA-regulated gene encoding an L-amino acid oxidase with broad substrate specificity in Aspergillus nidulans. Appl Environ Microbiol 71: 3551-3555.

Deshaies, R.J. (1999) SCF and Cullin/Ring H2-based ubiquitin ligases. Annu Rev Cell Dev Biol 15: 435-467.

Dever, T.E., Chen, J.J., Barber, G.N., Cigan, A.M., Feng, L., Donahue, T.F., London, I.M., Katze, M.G., and Hinnebusch, A.G. (1993) Mammalian eukaryotic initiation factor 2 alpha kinases functionally substitute for GCN2 protein kinase in the GCN4 translational control mechanism of yeast. Proc Natl Acad Sci U S A 90: 4616-4620.

Ebbole, D.J., Paluh, J.L., Plamann, M., Sachs, M.S., and Yanofsky, C. (1991) cpc-1, the general regulatory gene for genes of amino acid biosynthesis in Neurospora crassa, is differentially expressed during the asexual life cycle. Mol Cell Biol 11: 928-934.

Eckert, S.E., Hoffmann, B., Wanke, C., and Braus, G.H. (1999) Sexual development of Aspergillus nidulans in tryptophan auxotrophic strains. Arch Microbiol 172: 157-166.

Eckert, S.E., Kübler, E., Hoffmann, B., and Braus, G.H. (2000) The tryptophan synthase-encoding trpB gene of Aspergillus nidulans is regulated by the cross-pathway control system. Mol Gen Genet 263: 867-876.

Engelberg, D., Klein, C., Martinetto, H., Struhl, K., and Karin, M. (1994) The UV response involving the Ras signaling pathway and AP-1 transcription factors is conserved between yeast and mammals. Cell 77: 381 390. 
Fernandez, J., Lopez, A.B., Wang, C., Mishra, R., Zhou, L., Yaman, I., Snider, M.D., and Hatzoglou, M. (2003) Transcriptional control of the arginine/lysine transporter, cat-1, by physiological stress. J Biol Chem 278: 50000-50009.

Forsberg, H., Gilstring, C.F., Zargari, A., Martinez, P., and Ljungdahl, P.O. (2001) The role of the yeast plasma membrane SPS nutrient sensor in the metabolic response to extracellular amino acids. Mol Microbiol 42: 215-228.

Furuta, A., Takashima, S., Yokoo, H., Rothstein, J.D., Wada, K., and Iwaki, T. (2005) Expression of glutamate transporter subtypes during normal human corticogenesis and type II lissencephaly. Brain Res Dev Brain Res 155: 155-164.

Gagnadoux, F. (2005) [Invasive pulmonary aspergillosis complicating septic shock.]. Rev Mal Respir. Epub ahead of print

Galagan, J.E., Nusbaum, C., Roy, A., Endrizzi, M.G., Macdonald, P., FitzHugh, W., Calvo, S., Engels, R., Smirnov, S., Atnoor, D., Brown, A., Allen, N., Naylor, J., Stange-Thomann, N., DeArellano, K., Johnson, R., Linton, L., McEwan, P., McKernan, K., Talamas, J., Tirrell, A., Ye, W., Zimmer, A., Barber, R.D., Cann, I., Graham, D.E., Grahame, D.A., Guss, A.M., Hedderich, R., Ingram-Smith, C., Kuettner, H.C., Krzycki, J.A., Leigh, J.A., Li, W., Liu, J., Mukhopadhyay, B., Reeve, J.N., Smith, K., Springer, T.A., Umayam, L.A., White, O., White, R.H., Conway de Macario, E., Ferry, J.G., Jarrell, K.F., Jing, H., Macario, A.J., Paulsen, I., Pritchett, M., Sowers, K.R., Swanson, R.V., Zinder, S.H., Lander, E., Metcalf, W.W., and Birren, B. (2002) The genome of M. acetivorans reveals extensive metabolic and physiological diversity. Genome Res 12: 532-542.

Galagan, J.E., Calvo, S.E., Borkovich, K.A., Selker, E.U., Read, N.D., Jaffe, D., FitzHugh, W., Ma, L.J., Smirnov, S., Purcell, S., Rehman, B., Elkins, T., Engels, R., Wang, S., Nielsen, C.B., Butler, J., Endrizzi, M., Qui, D., Ianakiev, P., Bell-Pedersen, D., Nelson, M.A., Werner-Washburne, M., Selitrennikoff, C.P., Kinsey, J.A., Braun, E.L., Zelter, A., Schulte, U., Kothe, G.O., Jedd, G., Mewes, W., Staben, C., Marcotte, E., Greenberg, D., Roy, A., Foley, K., Naylor, J., Stange-Thomann, N., Barrett, R., Gnerre, S., Kamal, M., Kamvysselis, M., Mauceli, E., Bielke, C., Rudd, S., Frishman, D., Krystofova, S., Rasmussen, C., Metzenberg, R.L., Perkins, D.D., Kroken, S., Cogoni, C., Macino, G., Catcheside, D., Li, W., Pratt, R.J., Osmani, S.A., DeSouza, C.P., Glass, L., Orbach, M.J., Berglund, J.A., Voelker, R., Yarden, O., Plamann, M., Seiler, S., Dunlap, J., Radford, A., Aramayo, R., Natvig, D.O., Alex, L.A., Mannhaupt, G., Ebbole, D.J., Freitag, M., Paulsen, I., Sachs, M.S., Lander, E.S., Nusbaum, C., and Birren, B. (2003) The genome sequence of the filamentous fungus Neurospora crassa. Nature 422: 859-868.

Galagan, J.E., Calvo, S.E., Cuomo, C., Ma, L., Wortman, J., Batzoglou, S., Lee, S., Brudno, M., Bastürkmen, M., Spevak, C.C., Clutterbuck, J., Kapitonov, V., J., J., Scazzocchio, C., Farman, M., Butler, J., Purcell, S., Harris, S., Braus, G.H., Draht, O., Busch, S., D`Enfert, C., Bouchier, C., Goldman, G.H., GriffithsJones, S., Vienken, K., Pain, A., Selker, E.U., Archer, D., Penalva, M.Á., Oakley, B.R., Momany, M., Sano, M., Tanaka, T., Kumagai, T., Machida, M., Nierman, W.C., Denning, D.W., Caddick, M., Hynes, M., Paoletti, M., Fischer, E.R., Miller, J.B., Dyer, P., Sachs, M.S., Osmani, S.A., and Birren, B. (2005) Sequencing of Aspergillus nidulans and comparative analysis with A. fumigatus and A. oryzae. Manuscript submitted.

Glickman, M.H., Rubin, D.M., Coux, O., Wefes, I., Pfeifer, G., Cjeka, Z., Baumeister, W., Fried, V.A., and Finley, D. (1998) A subcomplex of the proteasome regulatory particle required for ubiquitin-conjugate degradation and related to the COP9-signalosome and eIF3. Cell 94: 615-623.

Goberdhan, D.C., Meredith, D., Boyd, C.A., and Wilson, C. (2005) PAT-related amino acid transporters regulate growth via a novel mechanism that does not require bulk transport of amino acids. Development 132: 2365-2375.

Gomez, E., Mohammad, S.S., and Pavitt, G.D. (2002) Characterization of the minimal catalytic domain within eIF2B: the guanine-nucleotide exchange factor for translation initiation. Embo J 21: 5292-5301. 
Gonzalez-Gonzalez, I.M., Cubelos, B., Gimenez, C., and Zafra, F. (2005) Immunohistochemical localization of the amino acid transporter SNAT2 in the rat brain. Neuroscience 130: 61-73.

Gugnani, H.C. (2003) Ecology and taxonomy of pathogenic aspergilli. Front Biosci 8: s346-357.

Hao, S., Sharp, J.W., Ross-Inta, C.M., McDaniel, B.J., Anthony, T.G., Wek, R.C., Cavener, D.R., McGrath, B.C., Rudell, J.B., Koehnle, T.J., and Gietzen, D.W. (2005) Uncharged tRNA and sensing of amino acid deficiency in mammalian piriform cortex. Science 307: 1776-1778.

Harding, H.P., Novoa, I., Zhang, Y., Zeng, H., Wek, R., Schapira, M., and Ron, D. (2000) Regulated translation initiation controls stress-induced gene expression in mammalian cells. Mol Cell 6: 1099-1108.

He, Q., Cheng, P., and Liu, Y. (2005) The COP9 signalosome regulates the Neurospora circadian clock by controlling the stability of the SCFFWD-1 complex. Genes Dev.

Hershko, A., and Ciechanover, A. (1998) The ubiquitin system. Annu Rev Biochem 67: 425-479.

Hilt, W. (2004) Targets of programmed destruction: a primer to regulatory proteolysis in yeast. Cell Mol Life Sci 61: $1615-1632$.

Hinnebusch, A.G. (1984) Evidence for translational regulation of the activator of general amino acid control in yeast. Proc Natl Acad Sci U S A 81: 6442-6446.

Hinnebusch, A.G. (1985) A hierarchy of trans-acting factors modulates translation of an activator of amino acid biosynthetic genes in Saccharomyces cerevisiae. Mol Cell Biol 5: 2349-2360.

Hinnebusch, A.G. (1986) The general control of amino acid biosynthetic genes in the yeast Saccharomyces cerevisiae. CRC Crit Rev Biochem 21: 277-317.

Hinnebusch, A.G. (1997) Translational regulation of yeast GCN4. A window on factors that control initiator-trna binding to the ribosome. J Biol Chem 272: 21661-21664.

Hinnebusch, A.G., and Natarajan, K. (2002) Gcn4p, a master regulator of gene expression, is controlled at multiple levels by diverse signals of starvation and stress. Eukaryot Cell 1: 22-32.

Hinnebusch, A.G., Asano, K., Olsen, D.S., Phan, L., Nielsen, K.H., and Valasek, L. (2004) Study of translational control of eukaryotic gene expression using yeast. Ann N Y Acad Sci 1038: 60-74.

Hoffmann, B., Mösch, H.U., Sattlegger, E., Barthelmess, I.B., Hinnebusch, A., and Braus, G.H. (1999) The WD protein $\mathrm{Cpc} 2 \mathrm{p}$ is required for repression of Gcn 4 protein activity in yeast in the absence of amino-acid starvation. Mol Microbiol 31: 807-822.

Hoffmann, B., Wanke, C., Lapaglia, S.K., and Braus, G.H. (2000) c-Jun and RACK1 homologues regulate a control point for sexual development in Aspergillus nidulans. Mol Microbiol 37: 28-41.

Hoffmann, B., Eckert, S.E., Krappmann, S., and Braus, G.H. (2001a) Sexual diploids of Aspergillus nidulans do not form by random fusion of nuclei in the heterokaryon. Genetics 157: 141-147.

Hoffmann, B., Valerius, O., Andermann, M., and Braus, G.H. (2001b) Transcriptional autoregulation and inhibition of mRNA translation of amino acid regulator gene cpcA of filamentous fungus Aspergillus nidulans. Mol Biol Cell 12: 2846-2857. 
Hutson, R.G., and Kilberg, M.S. (1994) Cloning of rat asparagine synthetase and specificity of the amino aciddependent control of its mRNA content. Biochem J 304 ( Pt 3): 745-750.

Hutson, R.G., Kitoh, T., Moraga Amador, D.A., Cosic, S., Schuster, S.M., and Kilberg, M.S. (1997) Amino acid control of asparagine synthetase: relation to asparaginase resistance in human leukemia cells. Am J Physiol 272: C1691-1699.

Hütter, R., and DeMoss, J.A. (1967) Organization of the tryptophan pathway: a phylogenetic study of the fungi. J Bacteriol 94: 1896-1907.

Hütter, R., Niederberger, P., and DeMoss, J.A. (1986) Tryptophan biosynthetic genes in eukaryotic microorganisms. Annu Rev Microbiol 40: 55-77.

Hyde, R., Taylor, P.M., and Hundal, H.S. (2003) Amino acid transporters: roles in amino acid sensing and signalling in animal cells. Biochem J 373: 1-18.

Irniger, S., and Braus, G.H. (2003) Controlling transcription by destruction: the regulation of yeast Gcn $4 p$ stability. Curr Genet 44: 8-18.

Jackson, P.K., and Eldridge, A.G. (2002) The SCF ubiquitin ligase: an extended look. Mol Cell 9: 923-925.

Jauniaux, J.C., and Grenson, M. (1990) GAP1, the general amino acid permease gene of Saccharomyces cerevisiae. Nucleotide sequence, protein similarity with the other bakers yeast amino acid permeases, and nitrogen catabolite repression. Eur J Biochem 190: 39-44.

Jensen, R., and Fischer, R. (1987) The postprephenate biochemical pathways to phenylalanine and tyrosine: an overview. Methods Enzymol 142: 472-478.

Jorgensen, M.U., Bruun, M.B., Didion, T., and Kielland-Brandt, M.C. (1998) Mutations in five loci affecting GAP1-independent uptake of neutral amino acids in yeast. Yeast 14: 103-114.

Jurka, J. (1998) Repeats in genomic DNA: mining and meaning. Curr Opin Struct Biol 8: 333-337.

Kapelari, B., Bech-Otschir, D., Hegerl, R., Schade, R., Dumdey, R., and Dubiel, W. (2000) Electron microscopy and subunit-subunit interaction studies reveal a first architecture of COP9 signalosome. J Mol Biol 300: 1169-1178.

Kapp, L.D., and Lorsch, J.R. (2004) The molecular mechanics of eukaryotic translation. Annu Rev Biochem 73: 657-704.

Kim, T., Hofmann, K., von Arnim, A.G., and Chamovitz, D.A. (2001) PCI complexes: pretty complex interactions in diverse signaling pathways. Trends Plant Sci 6: 379-386.

Klionsky, D.J. (2005) The molecular machinery of autophagy: unanswered questions. J Cell Sci 118: 7-18.

Kornitzer, D., Raboy, B., Kulka, R.G., and Fink, G.R. (1994) Regulated degradation of the transcription factor Gcn4. Embo J 13: 6021-6030.

Kouzarides, T., and Ziff, E. (1989) Behind the Fos and Jun leucine zipper. Cancer Cells 1: 71-76.

Krappmann, D., and Scheidereit, C. (2005) A pervasive role of ubiquitin conjugation in activation and termination of IkappaB kinase pathways. EMBO Rep 6: 321-326. 
Krappmann, S., Helmstaedt, K., Gerstberger, T., Eckert, S., Hoffmann, B., Hoppert, M., Schnappauf, G., and Braus, G.H. (1999) The aroC gene of Aspergillus nidulans codes for a monofunctional, allosterically regulated chorismate mutase. J Biol Chem 274: 22275-22282.

Krappmann, S., Bignell, E.M., Reichard, U., Rogers, T., Haynes, K., and Braus, G.H. (2004) The Aspergillus fumigatus transcriptional activator $\mathrm{CpcA}$ contributes significantly to the virulence of this fungal pathogen. Mol Microbiol 52: 785-799.

Kredics, L., Antal, Z., Szekeres, A., Hatvani, L., Manczinger, L., Vagvolgyi, C., and Nagy, E. (2005) Extracellular proteases of Trichoderma species. A review. Acta Microbiol Immunol Hung 52: 169-184.

Künzler, M., Balmelli, T., Egli, C.M., Paravicini, G., and Braus, G.H. (1993) Cloning, primary structure, and regulation of the HIS7 gene encoding a bifunctional glutamine amidotransferase: cyclase from Saccharomyces cerevisiae. J Bacteriol 175: 5548-5558.

Li, W., Wang, X., Van Der Knaap, M.S., and Proud, C.G. (2004) Mutations linked to leukoencephalopathy with vanishing white matter impair the function of the eukaryotic initiation factor $2 \mathrm{~B}$ complex in diverse ways. Mol Cell Biol 24: 3295-3306.

Luo, Z., Freitag, M., and Sachs, M.S. (1995) Translational regulation in response to changes in amino acid availability in Neurospora crassa. Mol Cell Biol 15: 5235-5245.

Magasanik, B., and Kaiser, C.A. (2002) Nitrogen regulation in Saccharomyces cerevisiae. Gene 290: 1-18.

Marzluf, G.A. (1997) Genetic regulation of nitrogen metabolism in the fungi. Microbiol Mol Biol Rev 61: 17-32.

McKenna, S., Moraes, T., Pastushok, L., Ptak, C., Xiao, W., Spyracopoulos, L., and Ellison, M.J. (2003) An NMR-based model of the ubiquitin-bound human ubiquitin conjugation complex Mms2.Ubc13. The structural basis for lysine 63 chain catalysis. J Biol Chem 278: 13151-13158.

Meijer, A.J., and Dubbelhuis, P.F. (2004) Amino acid signalling and the integration of metabolism. Biochem Biophys Res Commun 313: 397-403.

Meimoun, A., Holtzman, T., Weissman, Z., McBride, H.J., Stillman, D.J., Fink, G.R., and Kornitzer, D. (2000) Degradation of the transcription factor Gcn4 requires the kinase Pho85 and the SCF(CDC4) ubiquitinligase complex. Mol Biol Cell 11: 915-927.

Mielnicki, L.M., Hughes, R.G., Chevray, P.M., and Pruitt, S.C. (1996) Mutated Atf4 suppresses c-Ha-ras oncogene transcript levels and cellular transformation in NIH3T3 fibroblasts. Biochem Biophys Res Commun 228: 586-595.

Miller, J., and Gordon, C. (2005) The regulation of proteasome degradation by multi-ubiquitin chain binding proteins. FEBS Lett 579: 3224-3230.

Minehart, P.L., and Magasanik, B. (1992) Sequence of the GLN1 gene of Saccharomyces cerevisiae: role of the upstream region in regulation of glutamine synthetase expression. J Bacteriol 174: 1828-1836.

Mo, X., and Marzluf, G.A. (2003) Cooperative action of the NIT2 and NIT4 transcription factors upon gene expression in Neurospora crassa. Curr Genet 42: 260-267.

Mösch, H.U., Graf, R., Schmidheini, T., and Braus, G. (1990) Three GCN4 responsive elements act synergistically as upstream and as TATA-like elements in the yeast TRP4 promoter. Embo J 9: 29512957. 
Natarajan, K., Meyer, M.R., Jackson, B.M., Slade, D., Roberts, C., Hinnebusch, A.G., and Marton, M.J. (2001) Transcriptional profiling shows that Gen4p is a master regulator of gene expression during amino acid starvation in yeast. Mol Cell Biol 21: 4347-4368.

Nazar, R.N. (2004) Ribosomal RNA processing and ribosome biogenesis in eukaryotes. IUBMB Life 56: 457 465.

Nilsson, J., Sengupta, J., Frank, J., and Nissen, P. (2004) Regulation of eukaryotic translation by the RACK1 protein: a platform for signalling molecules on the ribosome. EMBO Rep 5: 1137-1141.

Nishida, H., Nishiyama, M., Kobashi, N., Kosuge, T., Hoshino, T., and Yamane, H. (1999) A prokaryotic gene cluster involved in synthesis of lysine through the amino adipate pathway: a key to the evolution of amino acid biosynthesis. Genome Res 9: 1175-1183.

Ogrydziak, D.M. (1993) Yeast extracellular proteases. Crit Rev Biotechnol 13: 1-55.

Omura, F., Kodama, Y., and Ashikari, T. (2001) The N-terminal domain of the yeast permease Bap2p plays a role in its degradation. Biochem Biophys Res Commun 287: 1045-1050.

Padbury, J.F., Diah, S.K., McGonnigal, B., Miller, C., Fugere, C., Kuzniar, M., and Thompson, N.L. (2004) Transcriptional regulation of the LAT-1/CD98 light chain. Biochem Biophys Res Commun 318: 529-534.

Paluh, J.L., Orbach, M.J., Legerton, T.L., and Yanofsky, C. (1988) The cross-pathway control gene of Neurospora crassa, cpc-1, encodes a protein similar to GCN4 of yeast and the DNA-binding domain of the oncogene v-jun-encoded protein. Proc Natl Acad Sci U S A 85: 3728-3732.

Paluh, J.L., and Yanofsky, C. (1991) Characterization of Neurospora CPC1, a bZIP DNA-binding protein that does not require aligned heptad leucines for dimerization. Mol Cell Biol 11: 935-944.

Pavlukova, E.B., Belozersky, M.A., and Dunaevsky, Y.E. (1998) Extracellular proteolytic enzymes of filamentous fungi. Biochemistry (Mosc) 63: 899-928.

Piotrowska, M., Natorff, R., and Paszewski, A. (2000) sconC, a gene involved in the regulation of sulphur metabolism in Aspergillus nidulans, belongs to the SKP1 gene family. Mol Gen Genet 264: 276-282.

Preiss, T., and Hentze, M.W. (2003) Starting the protein synthesis machine: eukaryotic translation initiation. Bioessays 25: 1201-1211.

Pries, R., Bömeke, K., Irniger, S., Grundmann, O., and Braus, G.H. (2002) Amino acid-dependent Gen4p stability regulation occurs exclusively in the yeast nucleus. Eukaryot Cell 1: 663-672.

Qiu, H., Hu, C., Yoon, S., Natarajan, K., Swanson, M.J., and Hinnebusch, A.G. (2004) An array of coactivators is required for optimal recruitment of TATA binding protein and RNA polymerase II by promoter-bound Gcn4p. Mol Cell Biol 24: 4104-4117.

Qu, L.H., Henry, Y., Nicoloso, M., Michot, B., Azum, M.C., Renalier, M.H., Caizergues-Ferrer, M., and Bachellerie, J.P. (1995) U24, a novel intron-encoded small nucleolar RNA with two $12 \mathrm{nt}$ long, phylogenetically conserved complementarities to 28S rRNA. Nucleic Acids Res 23: 2669-2676.

Quevillon-Cheruel, S., Leulliot, N., Meyer, P., Graille, M., Bremang, M., Blondeau, K., Sorel, I., Poupon, A., Janin, J., and van Tilbeurgh, H. (2004) Crystal structure of the bifunctional chorismate synthase from Saccharomyces cerevisiae. J Biol Chem 279: 619-625. 
Rohde, J.R., Campbell, S., Zurita-Martinez, S.A., Cutler, N.S., Ashe, M., and Cardenas, M.E. (2004) TOR controls transcriptional and translational programs via Sap-Sit4 protein phosphatase signaling effectors. Mol Cell Biol 24: 8332-8341.

Russnak, R., Konczal, D., and McIntire, S.L. (2001) A family of yeast proteins mediating bidirectional vacuolar amino acid transport. J Biol Chem 276: 23849-23857.

Sales, A.C., and Yoshizawa, T. (2005) Updated profile of aflatoxin and Aspergillus section Flavi contamination in rice and its byproducts from the Philippines. Food Addit Contam 22: 429-436.

Schmidt, A., Hall, M.N., and Koller, A. (1994) Two FK506 resistance-conferring genes in Saccharomyces cerevisiae, TAT1 and TAT2, encode amino acid permeases mediating tyrosine and tryptophan uptake. Mol Cell Biol 14: 6597-6606.

Schneper, L., Düvel, K., and Broach, J.R. (2004) Sense and sensibility: nutritional response and signal integration in yeast. Curr Opin Microbiol 7: 624-630.

Sengupta, J., Nilsson, J., Gursky, R., Spahn, C.M., Nissen, P., and Frank, J. (2004) Identification of the versatile scaffold protein RACK1 on the eukaryotic ribosome by cryo-EM. Nat Struct Mol Biol 11: 957-962.

Shemer, R., Meimoun, A., Holtzman, T., and Kornitzer, D. (2002) Regulation of the transcription factor Gcn4 by Pho85 cyclin PCL5. Mol Cell Biol 22: 5395-5404.

Shor, B., Calaycay, J., Rushbrook, J., and McLeod, M. (2003) Cpc2/RACK1 is a ribosome-associated protein that promotes efficient translation in Schizosaccharomyces pombe. J Biol Chem 278: 49119-49128.

Sirtori, L.R., Dutra-Filho, C.S., Fitarelli, D., Sitta, A., Haeser, A., Barschak, A.G., Wajner, M., Coelho, D.M., Llesuy, S., Bello-Klein, A., Giugliani, R., Deon, M., and Vargas, C.R. (2005) Oxidative stress in patients with phenylketonuria. Biochim Biophys Acta 1740: 68-73.

Sood, R., Porter, A.C., Olsen, D.A., Cavener, D.R., and Wek, R.C. (2000) A mammalian homologue of GCN2 protein kinase important for translational control by phosphorylation of eukaryotic initiation factor2alpha. Genetics 154: 787-801.

Sosa, E., Aranda, C., Riego, L., Valenzuela, L., DeLuna, A., Cantu, J.M., and Gonzalez, A. (2003) Gcn4 negatively regulates expression of genes subjected to nitrogen catabolite repression. Biochem Biophys Res Commun 310: 1175-1180.

Takeuchi, J., and Tamura, T. (2004) Recombinant ATPases of the yeast 26S proteasome activate protein degradation by the $20 \mathrm{~S}$ proteasome. FEBS Lett 565: $39-42$.

Thammarongtham, C., Turner, G., Moir, A.J., Tanticharoen, M., and Cheevadhanarak, S. (2001) A new class of glutaminase from Aspergillus oryzae. J Mol Microbiol Biotechnol 3: 611-617.

Tyers, M., and Jorgensen, P. (2000) Proteolysis and the cell cycle: with this RING I do thee destroy. Curr Opin Genet Dev 10: 54-64.

Valenzuela, L., Aranda, C., and Gonzalez, A. (2001) TOR modulates GCN4-dependent expression of genes turned on by nitrogen limitation. J Bacteriol 183: 2331-2334.

Valerius, O., Draht, O., Kübler, E., Adler, K., Hoffmann, B., and Braus, G.H. (2001) Regulation of hisHF transcription of Aspergillus nidulans by adenine and amino acid limitation. Fungal Genet Biol 32: 21-31. 
van Nocker, S., and Vierstra, R.D. (1993) Multiubiquitin chains linked through lysine 48 are abundant in vivo and are competent intermediates in the ubiquitin proteolytic pathway. J Biol Chem 268: 24766-24773.

Vazquez de Aldana, C.R., Marton, M.J., and Hinnebusch, A.G. (1995) GCN20, a novel ATP binding cassette protein, and GCN1 reside in a complex that mediates activation of the eIF-2 alpha kinase GCN2 in amino acid-starved cells. Embo J 14: 3184-3199.

Verrey, F., Jack, D.L., Paulsen, I.T., Saier, M.H., Jr., and Pfeiffer, R. (1999) New glycoprotein-associated amino acid transporters. J Membr Biol 172: 181-192.

Volterra, A., and Meldolesi, J. (2005) Astrocytes, from brain glue to communication elements: the revolution continues. Nat Rev Neurosci.

Wang, H., Wang, X., and Jiang, Y. (2003) Interaction with Tap42 is required for the essential function of Sit4 and type 2A phosphatases. Mol Biol Cell 14: 4342-4351.

Wanke, C., Eckert, S., Albrecht, G., van Hartingsveldt, W., Punt, P.J., van den Hondel, C.A., and Braus, G.H. (1997) The Aspergillus niger GCN4 homologue, CpcA, is transcriptionally regulated and encodes an unusual leucine zipper. Mol Microbiol 23: 23-33.

Wipf, D., Ludewig, U., Tegeder, M., Rentsch, D., Koch, W., and Frommer, W.B. (2002) Conservation of amino acid transporters in fungi, plants and animals. Trends Biochem Sci 27: 139-147.

Wittenberg, C., and Reed, S.I. (2005) Cell cycle-dependent transcription in yeast: promoters, transcription factors, and transcriptomes. Oncogene 24: 2746-2755.

Yelton, M.M., Hamer, J.E., de Souza, E.R., Mullaney, E.J., and Timberlake, W.E. (1983) Developmental regulation of the Aspergillus nidulans trpC gene. Proc Natl Acad Sci U S A 80: 7576-7580.

Zagami, C.J., O'Shea, R.D., Lau, C.L., Cheema, S.S., and Beart, P.M. (2005) Regulation of glutamate transporters in astrocytes: evidence for a relationship between transporter expression and astrocytic phenotype. Neurotox Res 7: 143-149.

Zhu, S., Sobolev, A.Y., and Wek, R.C. (1996) Histidyl-tRNA synthetase-related sequences in GCN2 protein kinase regulate in vitro phosphorylation of eIF-2. J Biol Chem 271: 24989-24994. 


\section{Chapter 4}

\section{A. nidulans CpcA localisation and nuclear localisation signal (NLS)}

\subsection{Abstract}

The yeast transcription factor Gen4p contains two nuclear localisation sequences NLS1 and NLS2 which are independently able to relocate the cytoplasmic protein chorismate mutase to the nucleus. Only NLS2 is highly conserved among other fungi. The counterpart of Gen $4 p$ in Aspergillus nidulans is CpcA. The conserved NLS of this transcription factor is necessary to target the protein into the nucleus in A. nidulans and in $S$. cerevisiae. Deletion of the NLS leads to accumulation of the protein in the cytoplasm in both organisms. This suggests that only one NLS is present in CpcA. During the manual annotation of the A. nidulans genome two putative ORFs SrpA (AN2142.1) and KapA (AN0906.1) were found that show high similarity to the $\alpha$-importin Srp1p and $\beta$-importin Kap95p which are necessary to transport Gen4p into the nucleus. 


\subsection{Introduction}

During protein production transcription and translation are spatially separated in eukaryotes. The transcription of the mRNA takes place in the nucleoplasm, whereas its translation takes place in the cytoplasm. The nucleoplasm and cytoplasm are spatially separated by the nuclear membrane, being continuous with the endoplasmatic reticulum and are connected by the nuclear pores. The pores are required for trafficking of involved macromolecules like mRNA, tRNA, rRNA or proteins as transcription factors of ribosome compounds and work in both directions (Kaffman and O'Shea, 1999; Lusk et al., 2004). Since all proteins are synthesized in the cytoplasm nuclear proteins need to be transported into the nucleus (Görlich and Mattaj, 1996). Small proteins up to $40 \mathrm{kDa}$ can generally diffuse passively through the nuclear pores, whereas bigger macromolecules need to be transported by special transporter proteins which allow an active, efficient and regulated import to the nucleus. In general active nuclear import requires the formation of an heterodimeric importin $\alpha / \beta$ complex in the cytosol which recognizes the nuclear localisation sequence (NLS) of the cargo protein and translocates it through the nuclear pore into the nucleus (Görlich et al., 1995). After release of the cargo protein the importins return to the cytosol. This active transport is driven by GTP hydroloysis by Ran (Görlich and Mattaj, 1996; Moore and Blobel, 1993).

Classical NLSs are either monopartite sequences that contain a single cluster of basic amino acids (Lys/Arg) or bipartite sequences that contain two clusters of basic residues separated by an unconserved linker region (Dingwall and Laskey, 1991). Only one importin $\alpha$ protein (Srp1p) is known in Saccharomyces cerevisiae, whereas thirteen proteins show similarities to importin $\beta$, eight of which being already characterized as import proteins. Gcn $4 p$ is the central transcription factor of the general control of amino acid biosynthesis in S. cerevisiae (Natarajan et al., 2001). It controls the expression of over 500 target genes belonging to various biosynthetic pathways under amino acid limitation, purine starvation, UV radiation or glucose limitation (Engelberg et al., 1994; Hinnebusch and Natarajan, 2002; Mösch et al., 1990). Starvation for amino acids has two effects on Gcn $4 p$, both the synthesis and the stability of this transcription factor are increased. Under non-limiting amino acid conditions the protein is highly instable with a half-life of approximately 5 minutes. The half-life increases to 20 minutes under limiting conditions (Kornitzer et al., 1994). As a transcription factor Gcn $4 \mathrm{p}$ has to be transported into the nucleus after synthesis in the cytoplasm (Pries $e t$ al., 2002). Gcn4p has two nuclear localisation sequences NLS1 and NLS2 which are both located near the C-terminus of the protein. Each of the NLS can misdirect proteins to the 
nucleus and deletion of the NLSs prevents Gcn4p from entering the nucleus (Pries et al., 2002). The two karyopherins Srp1p and Kap95p are essential to transport Gcn $4 p$ into the nucleus (Pries et al., 2004). Since the stability of Gcn4p seems to be only affected in the nucleus by phosphorylation and subsequent ubiquitination and degradation by the $26 \mathrm{~S}$ proteasome the small cytoplasmic portion of Gen $4 p$ is more stable than the nuclear fraction. Phosphorylation of Gcn $4 p$ in the nucleus requires the cyclin-dependent kinases Srb10p and Pho85p in combination with their cyclins Srb10p and Pcl5p (Chi et al., 2001; Meimoun et al., 2000). CpcA is the counterpart of Gen4p in the filamentous fungus Aspergillus nidulans and acts as the transcription factor of the cross-pathway control of amino acid biosynthesis (Hoffmann et al., 2001). So far nothing was known about the subcellular localisation of CpcA but alignments of the CpcA and the Gcn4p amino acid sequence revealed that only NLS2 is conserved among the two proteins. Furthermore it is the only NLS to be found in CpcA. Fluorescence microscopy experiments revealed that $\mathrm{CpcA}$ is nuclear localized if expressed in yeast and in Aspergillus nidulans. Deletion of the conserved NLS leads to accumulation of the protein in the cytoplasm, both in yeast and in Aspergillus. 


\subsection{Material and methods}

\subsubsection{Transformation and cultivation of Aspergillus nidulans}

For CpcA localisation analysis we used the A. nidulans strain AGB10 (pyrG89, pyroA4) from our collection, which is a derivative of wild type strain A4 (Eckert et al., 2000). Cultivation of A. nidulans strains was performed at $37^{\circ} \mathrm{C}$ on minimal medium. Transformation was carried out as previously described (Punt and van den Hondel, 1992), and transformants were selected on medium lacking uridine to select for the presence of the prototrophic marker pyrG. Expression of the alcA promoter was induced with $2 \%$ ethanol and $2 \%$ glycerol as sole carbon sources. The amount of integrations of the constructs into the genome was checked by southern experiments (data not shown). Strains with one integrated copy of the respective plasmids were further investigated.

\subsubsection{Fluorescence microscopy}

The microscopic experiments were carried out on a Zeiss Axiovert microscope by either differential interference contrast microscopy (DIC) or fluorescence microscopy using a GFP filter set (AHF Analysentechnik AG, Tübingen, Germany). Nuclei were stained with DAPI (4,6-diamidino-2-phenylindole) and visualized using a Hamamatsu-Orca ER digital camera and Improvision Openlab software (Improvision, Coventry, UK). For fluorescence microscopy of $A$. nidulans a thin layer of solid medium (without uridine to select for the presence of the prototrophic marker pyrG) was spread evenly on a glass slide, which was then tilted so that one end was in contact with a reservoir of liquid medium. The solid layer was inoculated with spores of the transformed A. nidulans strain and analyzed by fluorescence microscopy after formation of hyphae. 


\subsection{Results}

\subsubsection{Comparison of Gen4p and CpcA reveals that only NLS2 is conserved between Saccharomyces cerevisiae and Aspergillus species}

The yeast transcription factor Gcn $4 p$ contains two regions which are able to target the protein after translation in the cytosol towards the nucleus. Sequence alignments revealed that the NLS2 is highly conserved among homologous proteins of other fungi, such as the filamentous fungus Aspergillus nidulans (Figure 18A), A. fumigatus, A. niger and A. oryzae CpcA and the Neurospora crassa CPC-1. Even in the human AP-1 transcription factor c-jun a putative NLS can be found that shows a 58\% identity to Gcn4p (Figure 18B). No conserved NLS1 of Gcn $4 p$ could be found in any of the other investigated organisms. CpcA of A. nidulans is the transcription factor of the cross-pathway control, the counterpart of $S$. cerevisiae Gcn $4 p$ general control. Strong similarities can be found among these two proteins (40\% identity) and it was shown that they can functionally substitute for each other.

\subsubsection{Deletion of the conserved nuclear localisation sequence in $\mathrm{CpcA}$ of Aspergillus nidulans impairs its nuclear transport}

To investigate the subcellular localisation of $\mathrm{CpcA}$ in the filamentous fungus A. nidulans a GFP-tagged version of the protein was expressed from the ethanol inducible alcA promoter in strain AGB10 (pyrG89, pyroA4). A. nidulans expressing CpcA-GFP (pME2447) was grown on a glass slide on a thin layer of solid minimal medium lacking uridine for selection on the plasmid and containing $2 \%$ ethanol and $2 \%$ glycerol as sole carbon sources to induce the alcA promoter. Fresh hyphae could be directly investigated by fluorescence microscopy (Figure 19A). CpcA was found to be predominantly nuclear localized, as was confirmed by DAPI staining of the hyphae, which corresponds to the localisation recently described for Gcn4p. Expression of the truncated $\mathrm{CpcA}_{\mathrm{aa1}-190}$-GFP lacking the putative NLS from plasmid pME2448 leads to cytoplasmic accumulation of the protein which implies that the NLS is the sole functioning NLS in CpcA and thus implies that the protein lacks the counterpart to Gcn4p NLS1. To strengthen these results both GFP-tagged versions of CpcA were expressed in S. cerevisiae (Figure 19B). The untruncated chimeric protein (pME2494) shows clear nuclear localisation, whereas the truncated derivative $\mathrm{CpcA}_{\mathrm{aa1}-190}-\mathrm{GFP}$ (pME2495), which 
A

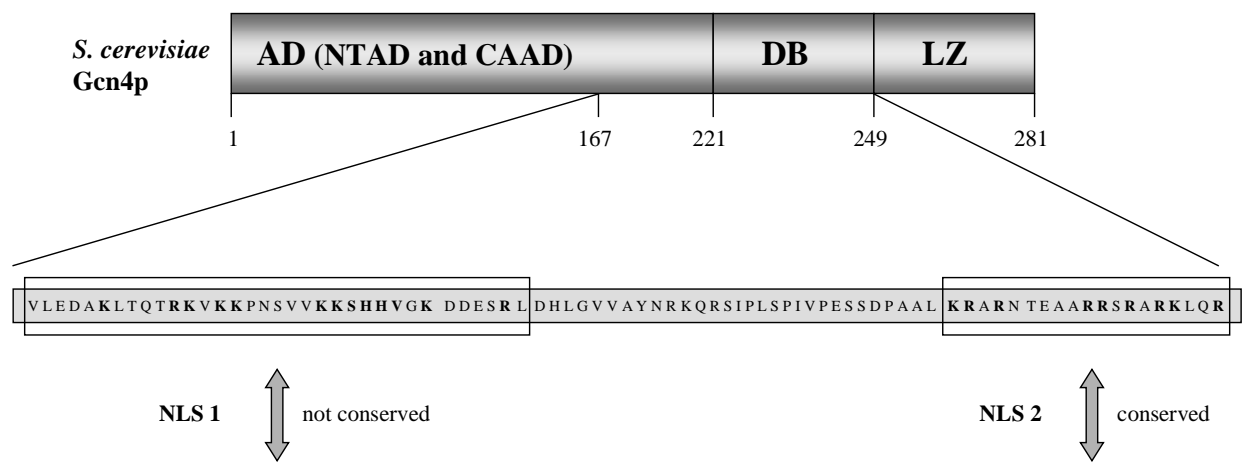

PTVSSPTVKPVSSPARSPTATSRSTTKHSTVAGVSARRTKPLPPIKYDESDPVAA KR ARNTEAARKS RARKLER

B

\begin{tabular}{|c|c|c|}
\hline & NLS2 & $\begin{array}{l}\% \text { Identity } \\
\text { to Gcn4p }\end{array}$ \\
\hline S. cerevisiae (Gcn4p) & KRARNTEAARRS RARKLQR & 100 \\
\hline A. nidulans $(\mathrm{CpcA})$ & KRARNTEAARKS RARKLER & 89,4 \\
\hline A. fumigatus (CpcA) & KRARNTEAARKS RARKLER & 89,4 \\
\hline A. niger (CpcA) & KRARNTEAARKS RARKLER & 89,4 \\
\hline A. oryzae (CpcA) & KRARNTEAARKS RARKLER & 89,4 \\
\hline N. crassa (CPC1) & KRARNTLAARKS $\underline{\text { RERKAQR }}$ & 78,9 \\
\hline H. sapiens (c-Jun) & KRMRNRIAASKC RKRKLER & 57,8 \\
\hline
\end{tabular}

Fig. 18: NLS motif comparisons

(A) The positions of the two NLS-motifs within the entire Gcn4 protein are shown schematically. NLS1 consists of the amino acids 167 to 200 and NLS2 is represented by the amino acids 231 to 249. The different domains of $\mathrm{Gcn} 4 \mathrm{p}$ are illustrated. $\mathrm{AD}$ is the activation domain, consisting of an $\mathrm{N}$-terminal activation domain (NTAD) and a central acidic activation domain (CAAD). DB is the DNA binding domain and LZ the leucine zipper of Gen4p.

An alignment with the amino acid sequence of $A$. nidulans CpcA reveals the conserved character of Gcn4p NLS2, whereas Gcn4p NLS1 is not conserved. Basic amino acid residues within the NLS motifs are written bold.

(B) Amino acid alignments of S. cerevisiae Gcn4p with the homologous proteins CpcA of Aspergillus species, CPC1 of Neurospora crassa, and human c-Jun revealed the highly conserved character of Gcn4p NLS 2.

lacks the nuclear localisation signal clearly accumulates in the cytoplasm as it does in A. nidulans. These data confirm that A. nidulans CpcA possesses only a single classical bipartite NLS motif, which is highly conserved among other fungi. 
A

GFP
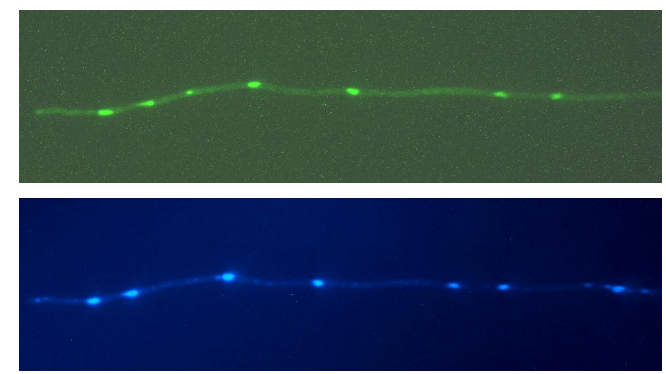

DAPI

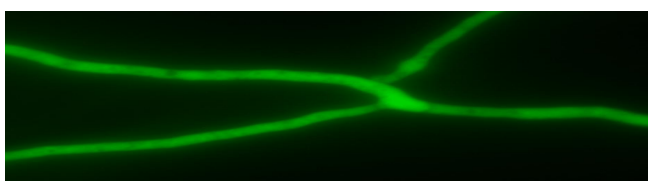

GFP

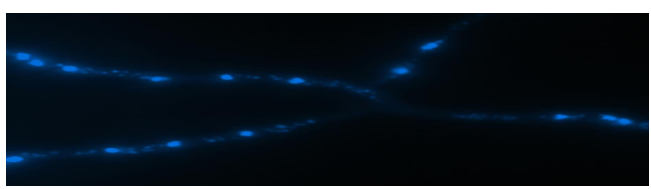

DAPI

\section{CpcA}

pME2447

\section{\begin{tabular}{l|l} 
CpcA & GFP \\
\hline
\end{tabular}}

pME2448

B

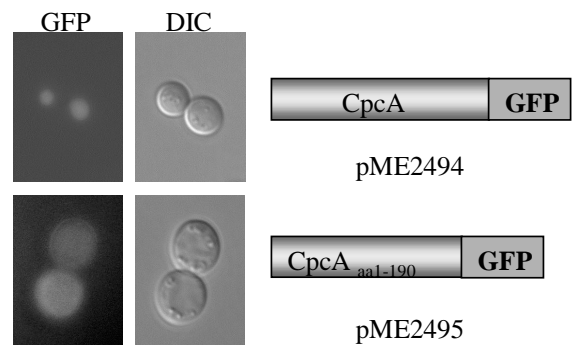

Fig. 19: Truncated $\mathrm{CpcA}_{\text {aa1-190 }}$ of $A$. nidulans accumulates in the cytoplasm

(A) Entire CpcA of $A$. nidulans was C-terminally conjugated with GFP and clearly nuclear localized, which was verified by DAPI staining of the nuclei. In contrast, truncated $\mathrm{CpcA}_{\mathrm{aa}-190}$ lacking the

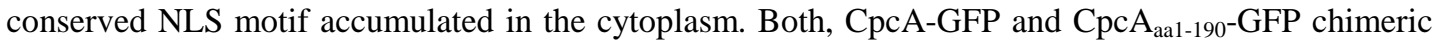
proteins were analyzed in Aspergillus nidulans AGB10 and driven from an inducible alcA promoter.

(B) Localization of entire and truncated CpcA was investigated in S. cerevisiae. CpcA of A. nidulans (pME2494) was localized in the nucleus of $S$. cerevisiae. Correspondingly to the cytoplasmic

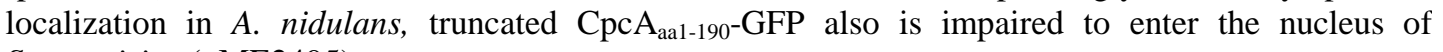
S. cerevisiae (pME2495). 


\subsubsection{In silico investigation on putative importins of Aspergillus nidulans}

Nuclear import is essential for Gcn4p to induce transcription of numerous genes whose products are involved in many different biosynthetic pathways in $S$. cerevisiae during response to amino acid starvation, glucose starvation and other stresses (Engelberg et al., 1994; Hinnebusch and Natarajan, 2002; Mösch et al., 1990; Natarajan et al., 2001). CpcA as counterpart of Gcn $4 p$ in A. nidulans needs to be transported to the nucleus, as well, to play its role as a transcription factor during amino acid starvation response (Pries et al., 2002). It was shown by Pries et al., 2004 that the $\alpha$-importin Srp1p and the $\beta$-importin Kap95p act as a heterodimer to channel Gcn $4 p$ into the yeast nucleus via the nuclear pores. So far no experimental procedures have been carried out to find whether similar proteins take over this task in A. nidulans or any other fungal species. Blast searches in the now available Aspergillus and $N$. crassa genomes revealed that proteins with high identities to Srp1p (over 58\%) and Kap95p (over 39\%) can be found to be encoded in the available Aspergillus and Neurospora crassa genomes (see Figure 20A and 20B). The assigned ORF for the Srp1p homologue SrpA was annotated as AN2142.1 and the Kap95p homologue KapA was annotated as AN0906.1 during the automated and manual annotation of the A. nidulans genome. Blast searches in the available Aspergillus nidulans genome revealed that there is only one $\alpha$-importin like protein and twelve members of the importin beta superfamily present, remarkingly this is one beta importin less than is present in S. cerevisiae. 


\subsection{Discussion}

\subsubsection{Nuclear import of the transcription factor CpcA}

It was recently shown that the entire yeast Gen 4 protein contains two nuclear localisation signals, each of which is able to direct a native cytoplasmic protein to the nucleus (Pries et al., 2002). So far the emergence of multiple nuclear localisation signals is unique in yeast, whereas the human transcription factor NF-AT2 is known to possess two NLS motifs and inactivation of both motifs is required to abolish nuclear import (Beals et al., 1997). The aim of this study was to investigate the capability of the A. nidulans CpcA NLS to guide the protein into the nucleus both in yeast, as well as in A. nidulans. The data presented in this work indicate that the single NLS of CpcA is necessary to guide the protein to the nucleus. This was observed expressing a GFP-tagged variant of the protein both in yeast and in A. nidulans. On the other hand the expressed truncated variants of $\mathrm{CpcA}$ accumulates in the cytoplasm in $S$. cerevisiae, as well as in its native host. This evidence lets us conclude that there is, unlike in yeast Gcn4p, only one NLS sufficient to target the CpcA protein to the nucleus and provides evidence that there is no hidden NLS1-like motive in CpcA. This is supported by the fact that we were unable to find a second NLS in the other investigated filamentous fungi. The nuclear localisation signal of the investigated $\mathrm{CpcA}$ proteins and yeast Gcn4p is well conserved, but nonetheless not identical. Though the experiments we carried out provides evidence, that the similarity of the bipartite NLS is high enough to properly target the transcription factor $\mathrm{CpcA}$ to the nucleus as well in bakers yeast, as in the filamentous fungus. Further experiments need to done to elucidate if $\mathrm{CpcA}$ is transported by Srp1p and Kap95p to the nucleus and if the putative proteins KapA and SrpA transport CpcA to the nucleus in A. nidulans. 


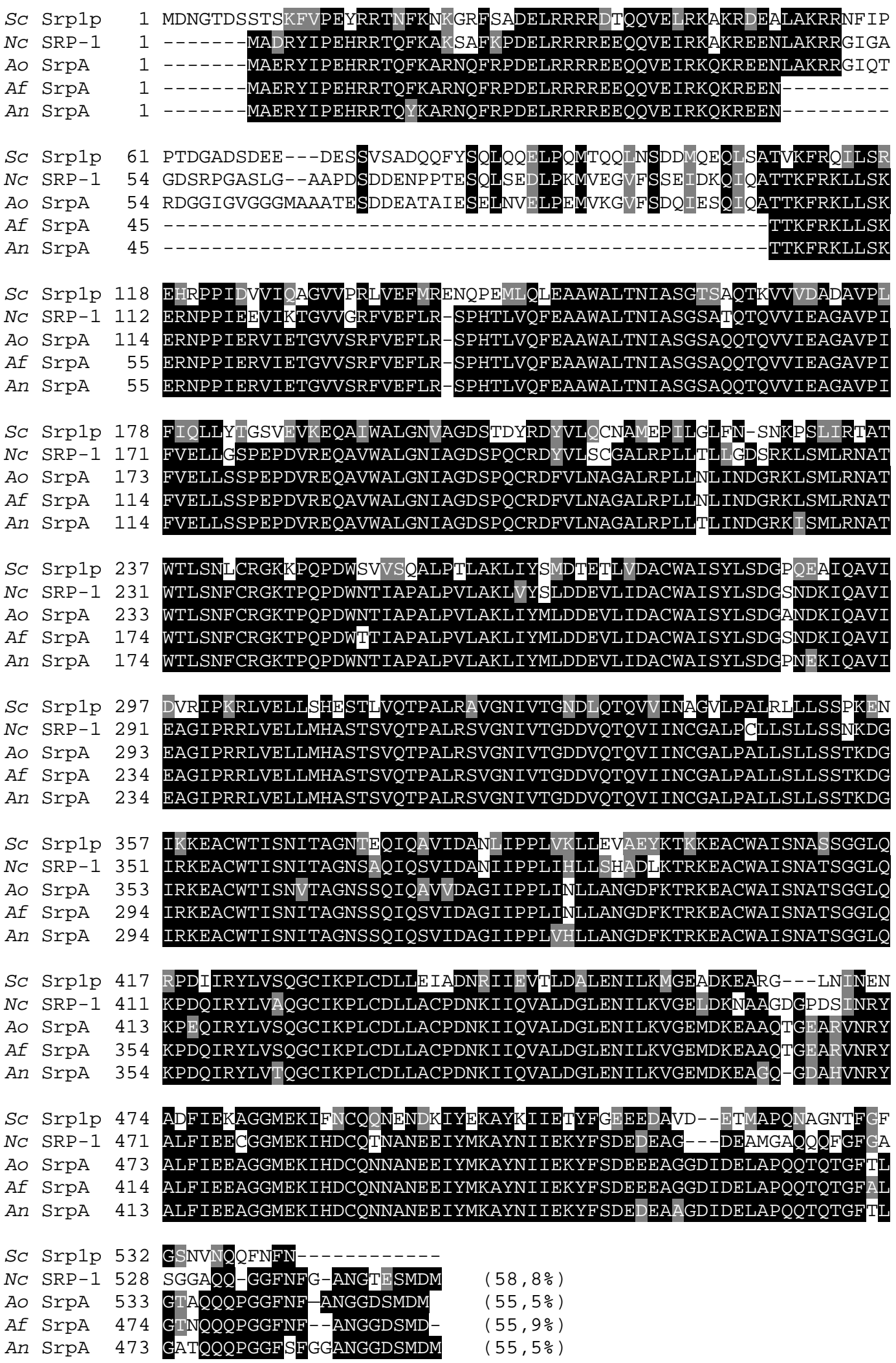

Fig. 20: Global multialignment of $\alpha$ and $\beta$ type karyophyrins (A)

The multialignment of fungal $\alpha$-importin Srp1p of $S$. cerevisiae ( $S c$ ), SrpA of A. nidulans (An), SrpA of A. fumigatus (Af), SrpA of A. oryzae (Ao) and srp-1 of $N$. crassa $(N c)$ shows a high conservation level. The percentage of identity of each single protein sequence to $S$. cerevisiae Srp1p is given in parentheses. 
SC Kap95p NC KAP-1

Ao KapA

Af KapA

An KapA

SC Kap95p

NC KAP-1

Ao KapA

Af KapA

An KapA

SC Kap95p

NC KAP-1

Ao KapA

Af $\mathrm{KapA}$

An KapA

SC Kap95p

NC KAP-1

Ao KapA

Af KapA

An KapA

SC Kap95p

NC KAP-1

Ao KapA

Af KapA

An KapA

SC Kap95p

NC KAP-1

Ao KapA

Af $\mathrm{KapA}$

An KapA

SC Kap95p

NC KAP-1

Ao KapA

Af KapA

An KapA

SC Kap95p

NC KAP-1

Ao KapA

Af KapA

An KapA

SC Kap95p

NC KAP-1

Ao KapA

Af KapA

An KapA

SC Kap95p

NC KAP-1

Ao KapA

Af KapA

An KapA

SC Kap95p

NC KAP-1

Ao KapA

Af KapA

An KapA

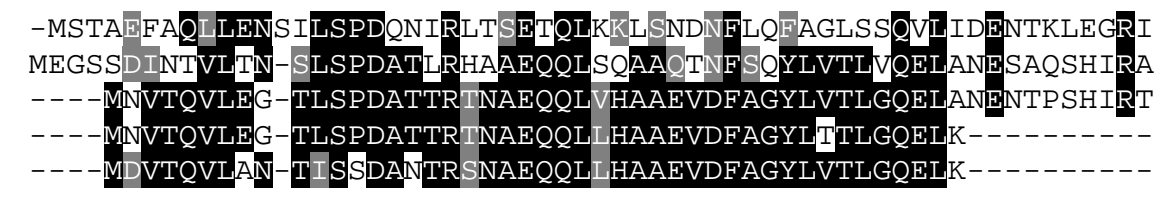

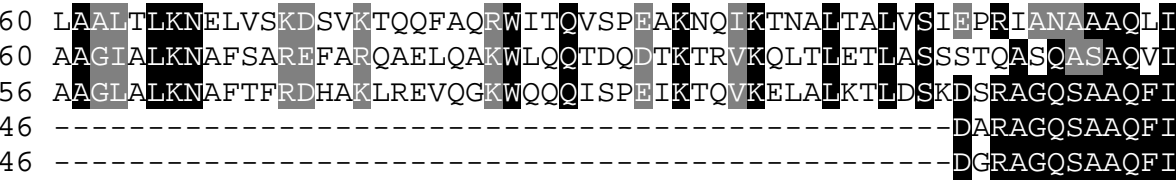

120 AAIADIELP HGAWP ELMKIMVDNT AAEQP ENVKRASLLALGYMCESADPQS-QALVSSSN 120 AA IATIELP RNEWP DLMHALVKNVSE-GSEHQKQASLTT I GF ICESQDVDLRNSLVQHSN 116 VSIAAIELPRNEWP DLMNVLVQNVAT-GSNQLKQASLITIGF ICESQDADLRESLTAHSN 58 VS IAAIELPRNEWP ELMNHLVQSVAT-GTDQLKQASLIT I GF ICESQDP ELRES LAAHSN 58 VSIAAIELPQNEWP DLMQ I LVQNVAS-GSDQMKQASLVT I GF ICESQEMELRES LAAHSN

179 NILIAIVQGAQSTETSKAVRLAALNALADS IF IKNNMEREGERNYLMQVVCEATQAED 179 AILTAVVQGARKEEPNREVRLAAITALGDSLEFVGNNFKHEGERNY IMQVICEATQAEDS 175 AILTAVVQGARREETNMD IRYAA I KALSD SVDFVRSNMDNEGERNY IMQVVCEATQADDI 117 AILTAVVQGARREEPNMD IRNAAIKALSDSVDFVRSNMENEGERNY IMQVVCEATQADDI 117 AILTAVVQGARREEQNMDIRFAAIKALSDSVDFVRSNMENEGERNY IMQVVCEATQAEDI

239 EVQAAAFGCLCK IMS LYYTF MKP YMEQALYALTIATMKSPNDKVASMTVEFWSTICEEE I 239 RIQQGAYGCLNR IMALYY ENMRFYMEKALFGLIILGMKSDDEDVAKLAVEFWSTVCEEE I 235 RVQAGAFGCLNR IMAAYYEKMRFYMEKALF GLS IMGMKSEEEDVAKLA IEFWC SVCEEE I 177 RVQAGAFGCLNRIMGSYYDKMRFYMEKALFGLS IMGMKSEEEDVAKLAIEFWCTVCEEEI 177 RVQAGAFGCLNRIMGAYYDKMSFFYMEKALFGLS IMGMKSEEEDVAKLA IEFWCTVCEEEI

299 DIAYEL--AQFPQSP--LQSYNFALSSIKDVVPNLLNLITRQNEDPEDDDWNVSMSAGAC 299 AIEDDN --AQVESSEQMRPFYNFARVATLEVVPVLLQLLTKQDEDAADDEYNISRAAYQC 295 AIEDDNAAAQAEGSP EVRP FFGFARVACREVVPVLLQAMCRQDEDATDDEYNVSRAAYQA 237 AIEDDNAAAQAEGATEIRPF FNFARVACREVVPVLLQCMCKQDEDATEDEYNISRAAYQA 237 AIEDDNAAAQAEGLTDVRPMYGFARIACREVVPVLLQAMCKQDEDAGDDEYNI SRAAYQA

355 LQLFAQNCGNHILEPVLEFVEONITADNWRNREAAVMAFGS IMDGP DKVQRTYYVHQALP 357 LQLYSQAVGAAI IQPVIQFVEANLRADDWHLRDAAVSAF GAMMD GP EEKLLEP IVKSGMQ 355 LQLYASCVQGEVIQPVLSFVEENIRNEDWRRRDAAVAAFGA IMD GP DPKVLEP LVKQALG 297 LQLYAQCVQ GD I IQPVLTFVEENIRNEDWRHRDAAVAAFGA IMD GP DPK I LEP LVKQALS 297 LQLYAQCVQADVIQPVLAFVEENIRSEDWRRRDAAVAAFGA IMD GP DP KVLEP LVKQALH

415 SILNLMNDQSLQVKETTAWCI GRIADSVAES IDPQQHLPGVVQACL IGLQDHPKVATNCS 417 PLIGMMEDP SLHVRD STAYALGRITETCSEVIDPAVHLDPLITSLFNGLMSSPRMAASCC 415 VLVGMMED SS IQVRD SAAYALGRVCDFCSETLDPDVHLQP LI SCLFNGLASTPKIASSCC 357 VLISMMED S IQVRD STAYALGRVCDFCSETLDP DVHLQP LI TCLFNGLAS SP K IASSCC 357 VLVSMMEDSS IQVRD SAAYALGRVCDFCSETLDP DVHLQP LI SCLFNGLASSP KIASSCC

475 WTIINLVEQLA---EATP SP IYNFYPALVDGLIGAANRIDNEFNARASAFSALTTMVEYA 477 WALMNLAERFGG Y GAAONP ITPHFNQCVTNLLAVTAKLDGDATVRTAAYEVLNVFVQNA 475 WALMNVADRFAGDVGAQTNP LSHFEESVKSLLTLTERQDADNQLRTAGYEVLNSFVTNA 417 WALMNVADRF AGDVGAHTNP LSKHF QD SVKSLLTLTERQDADNQLRTAGYEVLNSFVTNA 417 WALMNVADRFAGDVGAOTNP I SKYEEESVKSLLALTERSDADNQLRTAGYEVLNSFVTNA

532 TDTVAETSASISTFVMDKLGOTMSVDENQLTLEDAQSLQELQSNILTVLAAVIRKKSPSSVV 537 AND SLPAVASLSDVILQRLEETLPLQSQVVSVEDK I TLEDMQTSLCTVLQAI IQRLDKEI 535 AND SLPMVAS LSDVVIQRLEHT IPMQQQVVSVEDR I TLEEVQTS I SVI LAIVQRLETE I 477 ANDSLPLVATLSDVMIQRLEQT IPMQQQVVSVEDRI TLEEMQTSLTSVLLAIVQRLETEI 477 ANDSLPTVAHLSDVVLQRLERT IPMQQQVVSVEDRIMLEEMQTGITSVVLAIVQRLEAEI

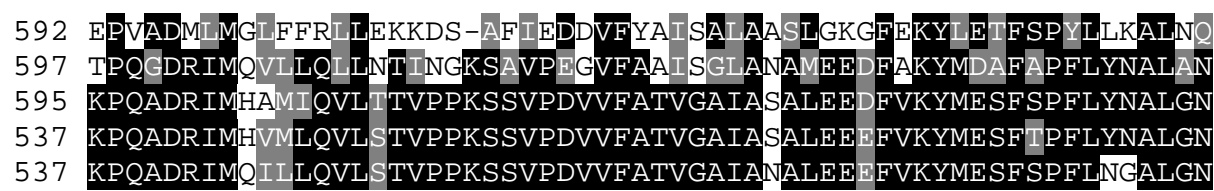




\begin{tabular}{|c|c|c|c|c|}
\hline$S C$ & Kap95p & 651 & VDSP-VSITAVGF IAD ISNSLEEDFRRYSDAMMNVLAQMISN & ARRELKPAVLSVFGDI \\
\hline NC & $\mathrm{KAP}-1$ & 657 & CSMA I GLVSD ITRSMGERSQPYCDQFMNYLLNNLRS & CFGD I \\
\hline Ao & KapA & 655 & GLVSD ISRALI & [LETFGDI \\
\hline Af & KapA & 597 & AIGLVSDIARALNEKVQP & TNQLKPAILETFGDI \\
\hline An & KapA & 597 & LCAMA IGLVSD I SRALNEKVLPYCDTFMNHLMNNLSS & ATNQLKPAILETFGD \\
\hline$S C$ & Kap95p & 710 & $2 \mathrm{II}$ & ב \\
\hline$N_{C}$ & $\mathrm{KAP}-1$ & 17 & YVISL & AWGGI I \\
\hline Ao & KapA & 713 & DYIVSL. & GIMDAWGGI \\
\hline Af & KapA & 655 & VTMEMLDYIVSL & GIMDAWGGILLTYKGK \\
\hline An & KapA & 55 & VTIEMLDYIISL & GIMDAWGGII \\
\hline$S C$ & Kap95p & 769 & & \\
\hline$N_{C}$ & KAP-1 & 777 & MGVIGD & PNGQ \\
\hline Ao & KapA & 773 & SMGVLGD & AFPNGEFA \\
\hline Af & KapA & 715 & RSEGLMRASMGVLGD & FPNGEF \\
\hline An & KapA & 715 & EGLMRASMGVLGI & G \\
\hline$S_{C}$ & Kap95p & 827 & GQLFSQATKDTARWAREQQKRQLS L----- & \\
\hline$N_{C}$ & $\mathrm{KAP}-1$ & 835 & EQVKRQI SGTQGMIQT & $(39,1 \%)$ \\
\hline Ao & KapA & 831 & TRNNRDFGATTVETARWAREQVKRQVTLSTAAAMA & $(39,1 \%)$ \\
\hline Af & KapA & 773 & VTALVRETRNNREYSART IDTARWTREQVKRQVNMSTAAAM- & $(35,2 \%)$ \\
\hline An & KapA & 774 & SLVRETRTNREYGQRT IDTARWAREQVKNQINMQGGGMS- & $(35,2 \%)$ \\
\hline
\end{tabular}

Fig. 20: Global multialignment of $\alpha$ and $\beta$ type karyophyrins (B)

The multialignment of fungal $\beta$-importin Kap95p of $S$. cerevisiae, KapA of A. nidulans, KapA of $A$. fumigatus, KapA of $A$. oryzae and kap-1 of $N$. crassa shows a high conservation level. The percentage of identity of each single protein sequence to $S$. cerevisiae Kap95p is given in parentheses. 


\subsubsection{Importins of Aspergillus nidulans}

Directed nuclear import of proteins requires energy and nuclear localisation signals and importins. In Pries et al., 2004 we have shown that the $\alpha$-importin Srp1p and the $\beta$-importin Kap95p are necessary to import Gcn4p into the nucleus of $S$. cerevisiae, here we are able to enlarge the scale of results with the help of in silico analyses of the A. nidulans genome. Srp1p is so far the only known member of the $\alpha$-importin family in $S$. cerevisiae and was found to recognize the bipartite NLS2 of Gcn4p and to trigger its nuclear import. Kap95p was shown to form heterodimers with Srp1p and in the publication evidence is provided that the $\beta$-karyopherin in combination with the $\alpha$-importin Srp $1 p$ is necessary to target $\operatorname{Gcn} 4 p$ to the nucleus (Pries et al., 2004). So far no experimental investigations were carried out, whether orthologs of these proteins play a similar role in A. nidulans. The availability to the A. nidulans genome and the deduced amino acid sequences made it possible to find first hints on these questions. Putative proteins with high similarity to both Srp1p and Kap95 can be found in A. nidulans, indicating that homologous proteins might play a similar role in nuclear uptake of the transcription factor CpcA. 


\subsection{References}

Beals, C.R., Clipstone, N.A., Ho, S.N., and Crabtree, G.R. (1997) Nuclear localization of NF-ATc by a calcineurin-dependent, cyclosporin-sensitive intramolecular interaction. Genes Dev 11: 824-834.

Chi, Y., Huddleston, M.J., Zhang, X., Young, R.A., Annan, R.S., Carr, S.A., and Deshaies, R.J. (2001) Negative regulation of Gen4 and Msn2 transcription factors by Srb10 cyclin-dependent kinase. Genes Dev 15: 1078-1092.

Dingwall, C., and Laskey, R.A. (1991) Nuclear targeting sequences - a consensus? Trends Biochem Sci 16: 478481.

Eckert, S.E., Kübler, E., Hoffmann, B., and Braus, G.H. (2000) The tryptophan synthase-encoding $\operatorname{trpB}$ gene of Aspergillus nidulans is regulated by the cross-pathway control system. Mol Gen Genet 263: 867-876.

Engelberg, D., Klein, C., Martinetto, H., Struhl, K., and Karin, M. (1994) The UV response involving the Ras signaling pathway and AP-1 transcription factors is conserved between yeast and mammals. Cell 77: 381-390.

Görlich, D., Vogel, F., Mills, A.D., Hartmann, E., and Laskey, R.A. (1995) Distinct functions for the two importin subunits in nuclear protein import. Nature 377: 246-248.

Görlich, D., and Mattaj, I.W. (1996) Nucleocytoplasmic transport. Science 271: 1513-1518.

Hinnebusch, A.G., and Natarajan, K. (2002) Gcn4p, a master regulator of gene expression, is controlled at multiple levels by diverse signals of starvation and stress. Eukaryot Cell 1: 22-32.

Hoffmann, B., Valerius, O., Andermann, M., and Braus, G.H. (2001) Transcriptional autoregulation and inhibition of mRNA translation of amino acid regulator gene $c p c A$ of filamentous fungus Aspergillus nidulans. Mol Biol Cell 12: 2846-2857.

Kaffman, A., and O'Shea, E.K. (1999) Regulation of nuclear localization: a key to a door. Annu Rev Cell Dev Biol 15: 291-339.

Kornitzer, D., Raboy, B., Kulka, R.G., and Fink, G.R. (1994) Regulated degradation of the transcription factor Gcn4. Embo J 13: 6021-6030.

Lusk, C.P., Makhnevych, T., and Wozniak, R.W. (2004) New ways to skin a kap: mechanisms for controlling nuclear transport. Biochem Cell Biol 82: 618-625.

Meimoun, A., Holtzman, T., Weissman, Z., McBride, H.J., Stillman, D.J., Fink, G.R., and Kornitzer, D. (2000) Degradation of the transcription factor Gen4 requires the kinase Pho85 and the SCF(CDC4) ubiquitinligase complex. Mol Biol Cell 11: 915-927.

Moore, M.S., and Blobel, G. (1993) The GTP-binding protein Ran/TC4 is required for protein import into the nucleus. Nature 365: 661-663.

Mösch, H.U., Graf, R., Schmidheini, T., and Braus, G. (1990) Three GCN4 responsive elements act synergistically as upstream and as TATA-like elements in the yeast TRP4 promoter. Embo J 9: 29512957. 
Natarajan, K., Meyer, M.R., Jackson, B.M., Slade, D., Roberts, C., Hinnebusch, A.G., and Marton, M.J. (2001) Transcriptional profiling shows that Gcn $4 \mathrm{p}$ is a master regulator of gene expression during amino acid starvation in yeast. Mol Cell Biol 21: 4347-4368.

Pries, R., Bömeke, K., Irniger, S., Grundmann, O., and Braus, G.H. (2002) Amino acid-dependent Gen4p stability regulation occurs exclusively in the yeast nucleus. Eukaryot Cell 1: 663-672.

Pries, R., Bömeke, K., Draht, O., Künzler, M., and Braus, G.H. (2004) Nuclear import of yeast Gen4p requires karyopherins Srp1p and Kap95p. Mol Genet Genomics 271: 257-266.

Punt, P.J., and van den Hondel, C.A. (1992) Transformation of filamentous fungi based on hygromycin B and phleomycin resistance markers. Methods Enzymol 216: 447-457. 



\section{Acknowledgements}

In the first place I would like to thank Professor Gerhard Braus for the supervision of this work. His ideas, optimism and understanding helped me a lot to find a way over the sticks and stones in the way of a scientist.

I thank PD Dr. Stefan Irniger for accepting to co-examine this thesis.

I especially thank Elke Schwier, who contributed with her diploma work to this thesis. I enjoyed the pleasant co-work with Ralph Pries and Katrin Bömeke during the course of examining the localisation of CpcA in Aspergillus and yeast.

A big thank also goes to the other members of the COP9-group Silke Busch, Krystyna Nahlik and Elke Schwier for discussion and concerted progression on the field of Aspergillus COP9 research.

Thanks to my current and former lab-members Sven Krappmann for great support during the course of this thesis, the sedulous Özgür Bayram for many discussions on the weekends, Verena Grosse for her competence on handling Aspergillus, Kerstin Helmstaedt whom I admire for her strong will and purposefulness and finally Christoph Sasse and Karen Meng for countless coffee-breaks and not always scientific discussions.

Thanks to all the former and present members of this group: the Marburgers: Hans-Ueli Mösch, Stefan Brückner, Melanie Bolte, Anne Obermayer; Patrick Dieckhoff, Olav Grundmann, Markus Hartmann, Axel Strittmatter, Andrea Pfeil, Gabi Heinrich, Claudia Fischer, Malte Kleinschmidt, Verena Pretz, Oliver Valerius, Lars Fichtner, Maria Meyer, Stefan Seiler, Nico Vogt, Nagela Rabanizada, Nirmala Padmanabhan, Fatih Sari, Heidi Northemann and Nicole Scheiter in administration and the technical staff Olaf Waase, Gerd Birke and Patrick Regin.

Finally I want to thank my family and friends who gave me a lot of general support all the time.

The Deutsche Forschungsgemeinschaft, the Fond der chemischen Industrie and the Volkswagen-Stiftung have granted financial support for this work. 



\title{
Curriculum vitae
}

\author{
Oliver Draht \\ born on December the $14^{\text {th }}, 1973$ \\ in Helmstedt, Germany
}

\section{Education}

\begin{abstract}
1980 - 1987 Primary Education in Rottorf and Königslutter, Germany
1987 - 1993 Secondary Education at the Gymnasium Anna-Sophianeum in Schöningen, Germany
\end{abstract}

\section{Scientific Background}

Oct. 1994 - Jul. 1999

Study of biology at the Georg-August Universität in Göttingen, Germany

Graduation in Microbiology with the diploma thesis "Charakterisierung des hisHF-Gens, welches für die Gluthamin Amidotransferase:Zyklase von Aspergillus nidulans codiert" at the Institute of Microbiology and Genetics, Department of Molecular Microbiology and Genetics of Prof. Dr. G.H. Braus

Aug. - Oct. 1999

Practical work for two months at the AO-Research Institute in Davos, Switzerland

Nov. 1999 - May $2000 \quad$ Practical work for six months in the laboratory of Prof. Dr. M.J. Hynes at the University of Melbourne, Australia, at the Department of Genetics

from June 2000

Scientific assistant at the Georg-August-Universität Göttingen, Germany, in the Institute of Microbiology and Genetics, Department of Molecular Microbiology and Genetics of Prof. Dr. G.H. Braus 